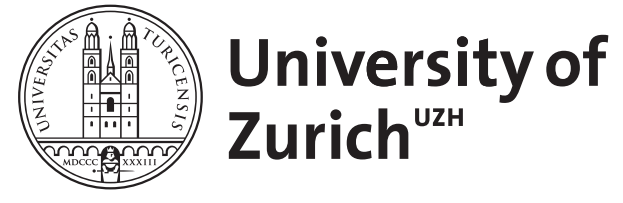

\title{
Les tribus amurrites de Mari
}

\author{
Anbar, Moshé
}

\begin{abstract}
A Tell Harīrī sur le Moyen-Euphrate, site de l'ancienne ville de Mari, 27 saisons de fouilles ont été dirigées jusqu'à maintenant, au cours desquelles 20000 tablettes et fragments de tablettes ont été trouvés, la plupart datant du milieu du XVIIIe siècle avant notre ère, l'époque de Hammurabi, roi de Babylonie. L'espace géographique qui forme le cadre où se sont déroulés les événements décrits dans ces documents va du golfe Persique jusqu'à la Méditerranée. L'espace qu'ils décrivent est riche en événements et en vicissitudes politiques, auxquels ont pris part des dizaines de grands et de petits rois, des rois de grands royaumes et de petites villes-royaumes. Dans les documents se reflètent à la fois les événements de grande portée et la vie journalière de la couche gouvernante ainsi que des simples citoyens. Les archives de Mari contiennent par ailleurs une riche information sur des tribus semi-nomades, qui appartiennent à la branche ouest des peuples sémitiques, qualifiés d'Amurrites. L'étude commence par une revue de l'histoire de la recherche sur les tribus amurrites puis décrit la chronologie et l'histoire de Mari à l'époque des dynasties amurrites. Par la suite sont examinés les divers aspects qui concernent ces tribus, à savoir leur organisation, leur distribution géographique, leurs dirigeants, leur économie, leurs relations avec les autorités et enfin leur origine.
\end{abstract}

Posted at the Zurich Open Repository and Archive, University of Zurich

ZORA URL: https://doi.org/10.5167/uzh-139839

Monograph

Published Version

Originally published at:

Anbar, Moshé (1991). Les tribus amurrites de Mari. Freiburg, Switzerland / Göttingen, Germany: Universitätsverlag / Vandenhoeck Ruprecht. 


\section{ORBIS BIBLICUS ET ORIENTALIS}

Publié au nom de I'Institut Biblique

de l'Université de Fribourg Suisse,

du Seminar für biblische Zeitgeschichte

der Universität Münster i.W.

et de la Société Suisse

pour l'Etude du Proche Orient Ancien

par Othmar Keel

avec la collaboration de

Erich Zenger et Albert de Pury

L'auteur:

Moshé Anbar, né à Ramat-Gan, près de Tel-Aviv, en Israël, a fait ses études bibliques et assyriologiques pour les degrés de M.A. et de licence aux Universités de Tel-Aviv, Jérusalem et Liège (1957-1966). Il a soutenu une thèse de doctorat en assyriologie dans le cadre de l'Université de Liège en 1971. II est professeur de Bible et d'assyriologie à I'Université de Tel-Aviv. II a publié de nombreuses études bibliques et assyriologiques. 


\section{Orbis Biblicus et Orientalis $\quad 108$}

Moshé Anbar

\section{Les tribus amurrites \\ de Mari}

Universitätsverlag Freiburg Schweiz Vandenhoeck \& Ruprecht Göttingen 
Die Deutsche Bibliothek - CIP-Einheitsaufnahme

\section{Anbar, Moshé}

Les tribus amurrites de Mari / Moshé Anbar.

Freiburg, Schweiz: Univ.-Verl.; Göttingen:Vandenhoeckund Ruprecht, 1991

(Orbis biblicus et orientalis; 108)

ISBN 3-7278-0750-4 (Univ.-Verl.)

ISBN 3-525-53741-7 (Vandenhoeck und Ruprecht)

NE: GT

Publié avec l'aide de l'Académie suisse

des sciences humaines

Die Druckvorlagen wurden vom Herausgeber als reprofertige Dokumente zur Verfügung gestellt

(C) 1991 by Universitätsverlag Freiburg Schweiz Vandenhoeck \& Ruprecht Göttingen

Paulusdruckerei Freiburg Schweiz

ISBN 3-7278-0750-4 (Universitätsverlag)

ISBN 3-525-53741-7 (Vandenhoeck \& Ruprecht)

Digitalisat erstellt durch Florian Lippke, Departement für

Biblische Studien, Universität Freiburg Schweiz 
A la mémoire de mes parents 



\section{Table des matières}

\section{Introduction}

1. Mari

2.L'histoire de la recherche sur les tribus amurrites de Mari $\quad$............................. 9

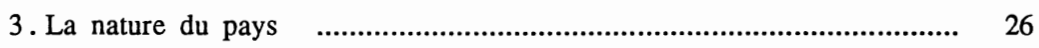

Chapitre I: La chronologie de Mari

1. L'époque des anciens rois …............................................................. 29

2. L'époque des gouverneurs ............................................................ 30

3.L'époque de la dynastie "Lim" et l'époque de Samsi-Addu - Yasmahn-addu ..... 31

A. Zimri-Lim .................................................................... 31

B. Yasmah-Addu - Samsi-Addu (................................................... 34

C. Sūmu-Yamam ................................................................. 35

D. Yahdun-Lim ….............................................................. 36

E. Résumé .................................................................. 37

Chapitre II: L'histoire de Mari à l'époque de la dynastie "Lim" et à l'époque de Samsi-Addu - Yasmah-Addu

1. Yahdun-Lim (1806/1805-1796/1795 avant notre ère) $\quad$.............................. 39

2. Sümu-Yamam (1795/1794-1793/1792) …............................................ 42

3. Yasmah-Addu (1793/1792-1776/1775) …........................................... 43

4. Zimri-Lim (1775/1774-1761/1760) ….............................................. 56

\section{Chapitre III: L'organisation tribale}

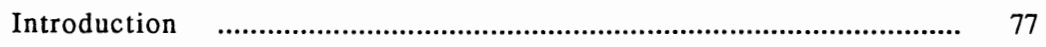

1. Hanûm …................................................................................ 80

2. Bini-Yamina ….................................................................. 83

3. Bini-Sim 'ål …................................................................... 85

4. Hanûm, Bini-Yamina et Bini-Sim'ål …............................................ 86

5. Sutûm …............................................................................... 88

6.L'analyse des noms propres des membres des trois grandes tribus $\quad \ldots . . . . . . . . . . \quad 89$ 


\section{Chapitre IV: La distribution géographique}

1. Le district de Mari …...................................................................... 91

A . Hanûm ............................................................................ 91

B. Bini-Yamina .................................................................... 92

C. Bini-Sim'al _...................................................................... 93

2. Le district de Terqa _................................................................... 94

A . Hanûm .............................................................................. 94

B . Bini-Yamina …...................................................................... 94

C. Bini-Sim'àl ........................................................................ 97

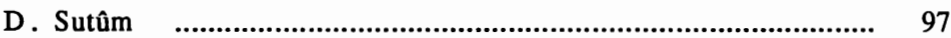

E. Numhă et Yamutbal .............................................................. 97

3. Le district de Saggarătum .................................................................. 97

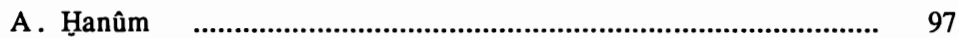

B . Bini-Yamina ….................................................................... 98

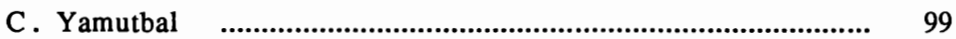

4. L'Ah-Purattim ................................................................................ 100

A. Hanûm

B . Bini-Yamina

C. Bini-Sim'àl …..................................................................... 100

5. Le district de Qattunăn $\quad$....................................................................... 101

A. Hanûm

B. Bini-Yamina …................................................................. 102

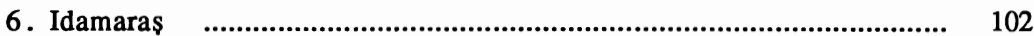

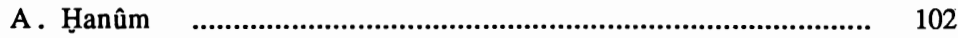

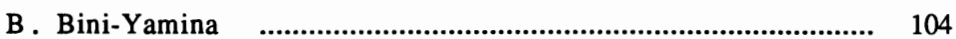

C. Bini-Sim'ål ….................................................................... 105

D. Yamutbal

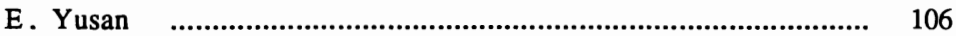

7. La région d'amont …...................................................................... 106

A. Hanûm

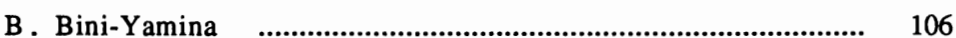

C. Bini-Sim'āl ...................................................................... 109

8. Zalmaqum ......................................................................... 109

A . Bini-Yamina ….................................................................. 109

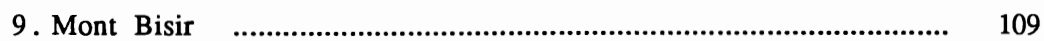

A. Bini-Yamina ….................................................................. 109

B. Sutûm ….................................................................... 110 
10. Yamhad, Qatanum et Amurrum _.................................................... 110

A. Bini-Yamina …................................................................... 110

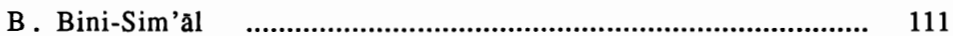

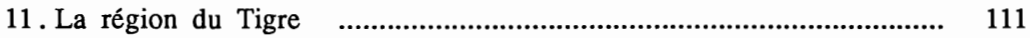

A. Bini-Yamina …................................................................ 111

B. Numhă $\quad$............................................................................... 112

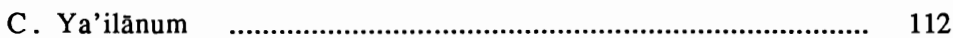

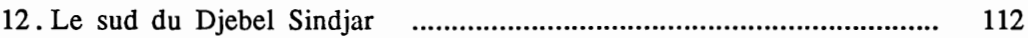

A. Hanûm

B. Numḩã et Yamutbal _............................................................ 113

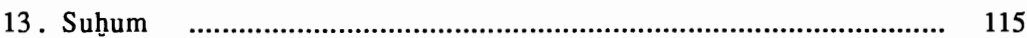

A. Hanûm $\quad$ ……...................................................................... 115

B . Bini-Yamina …............................................................. 115

C. Sutûm …….................................................................... 115

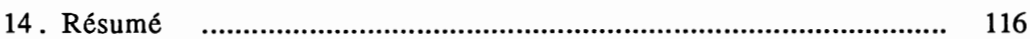

A. Ḩanûm ………....................................................................... 116

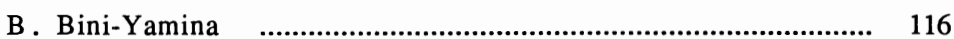

C. Bini-Sim'āl ……................................................................... 117

D. Sutûm

E. Numha et Yamutbal _........................................................... 117

F. Ya'ilānum …........................................................................ 117

\section{Chapitre V: Les dirigeants tribaux}

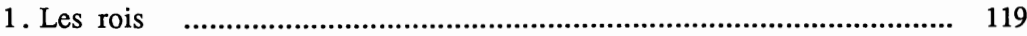

A. Les rois des Hanéens $\quad$.......................................................... 119

B. Les rois des Bini-Yamina …..................................................... 120

C. Un roi des Numhā

D. Conclusion ….................................................................... 131

2. Le madārum …........................................................................... 132

3. Le šãpiṭum ............................................................................. 133

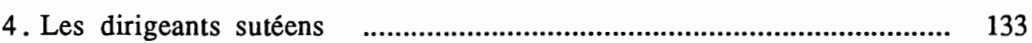

5. Les dirigeants numhéens …...................................................... 134

6. Le sugāgum _......................................................................... 134

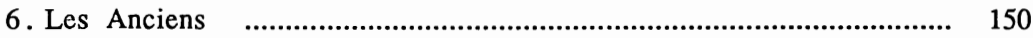

7. Le Hazannum ............................................................................. 155

8. L'assemblée (.......................................................................... 156 
Chapitre VI: L'économie des tribus

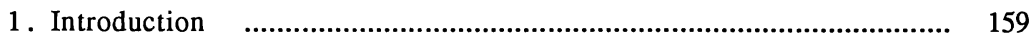

2. Le pâturage …........................................................................... 161

3. L'Agriculture …..................................................................... 170

A. Hanûm ............................................................................. 170

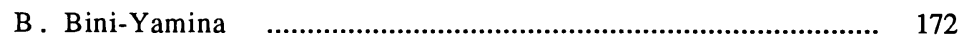

C. Bini-Sim'āl …................................................................. 174

D. Numbā

E. Résumé …......................................................................... 174

4. Diverses activités _....................................................................... 174

\section{Chapitre VII: Les relations entre les tribus et les autorités}

1. Hanûm …................................................................................... 177

A. L'époque de Yahdun-Lim _.................................................. 177

B.L'époque de Yasmahn-Addu - Samsi-Addu …................................. 177

C.L'époque de Yasmahn-Addu - Išme-Dagān $\quad$...................................... 180

D. L'époque de Zimri-Lim _........................................................ 181

2. Bini-Yamina ….................................................................. 191

A. L'époque de Yahdun-Lim _...................................................... 191

B. L'époque de Yasmah-Addu $\quad$...................................................... 191

C. L'époque de Zimri-Lim _.......................................................... 193

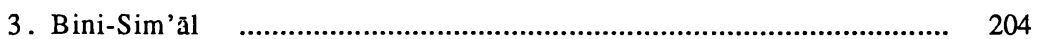

A. L'époque de Yahdun-Lim _..................................................... 204

B. L'époque de Yasmah-Addu ..................................................... 204

C. L'époque de Zimri-Lim ........................................................... 204

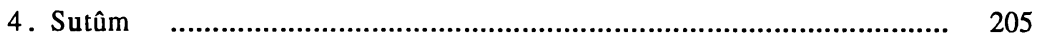

A. L'époque de Yahdun-Lim _........................................................ 205

B. L'époque de Yasmah-Addu ....................................................... 205

C. L'époque de Zimri-Lim _.......................................................... 205

5. Yamutbal _............................................................................ 207

A. L'époque de Zimri-Lim _....................................................... 207

6. Numbā _.................................................................................. 207

A. L'époque de Yahdun-Lim _......................................................... 207

B. L'époque de Zimri-Lim _................................................... 207

Chapitre VIII: L'origine des tribus amurrites f.................................... 209 
Abréviation s .......................................................................................... 221

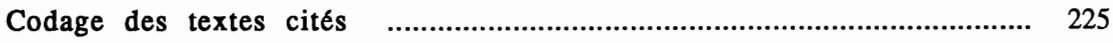

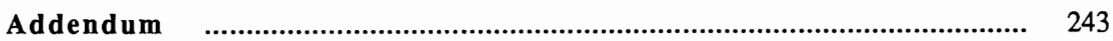

Cartes

1. Une carte des régions et des précipitations ……...................................... 28

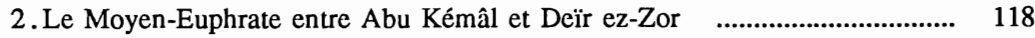

3. La Babylonie ........................................................................ 217

4. Une carte détaillée .............................................................. 218 - 219

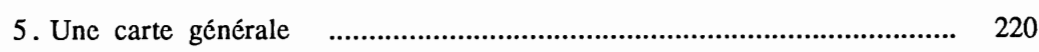





\section{Avant-propos}

En 1985 nous avons publié en hébreu un ouvrage intitulé Les tribus amurrites de Mari et l'installation des Fils d'Israil en Canaan. Le présent ouvrage n'est pas seulement une traduction de la version hébraïque, mais une véritable nouvelle édition. Dans l'étude de Mari les nouveautés s'accumulent d'un jour à l'autre, et nous nous sommes même posé la question: ne doit-on pas attendre de nouvelles publications par l'équipe si active de Mari ? Mais nous avons décidé qu'à un moment donné, il faut s'arrêter sachant que de nouvelles données viendront sans doute jeter une nouvelle lumière sur nombre de questions que nous avons traitées dans notre étude. Le but de celle-ci est de mettre à la disposition du lecteur, qu'il soit assyriologue, historien du Proche Orient ancien, bibliste, sociologue ou anthropologue, toutes les données mariotes sur les tribus amurrites de Mari connues jusqu'à la date de la remise du manuscrit à l'éditeur, organisées d'une manière simple et claire. [voir Addendum signalé par un astérisque $(*)$ ].

C'est avec un grand plaisir que nous voulons remercier notre ancien Maître le Professeur J.-R. Kupper, l'auteur de l'ouvrage fondamental sur les nomades à Mari et en Mésopotamie en général, pour avoir eu l'amabilité de lire notre manuscrit, et de nous faire part de ses précieuses remarques et observations.

De même nous voulons remercier le Professeur O. Keel d'avoir accepté notre ouvrage pour publication dans la série Orbis biblicus et orientalis, le $\mathrm{Dr} \mathrm{Ch}$. Uehlinger pour le soin qu'il a apporté à l'édition de l'ouvrage et le Professeur D. Charpin pour sa fonte "Cunanlaser".

Pour des raisons techniques, il nous était impossible de signaler les centaines et même milliers de corrections (relectures et collations) et nouvelles interprétations qui ont suivi la publication des premières éditions.

Pour les noms propres et les noms géographiques, nous avons unifié leurs formes, n'utilisant que la forme devenue classique ou celle qui nous a semblé être la plus courante.

M. A. 



\section{Introduction}

\section{Mari}

L'histoire de la découverte de Mari commence lors du mandat français en Syrie. Un jour, au début du mois d'août de l'année 1933, on a découvert par hasard à Tell Hुarīī sur le Moyen-Euphrate une statue qui portait une inscription cunéiforme. ${ }^{1}$ La statue a été transportée au Musée d'Alep, et la nouvelle de la découverte est parvenue à René Dussaud, le Conservateur des Antiquités orientales au Musée du Louvre. R. Dussaud s'est hâté d'organiser une mission archéologique qui devait partir pour mener des fouilles sur le site où avait été trouvée la statue. Et en effet, la même année une mission archéologique, dirigée par André Parrot, partait pour fouiller le Tell Ḩarīīi. Les fouilles ont commencé au milieu du mois de décembre 1933, et après quarante jours, la mission a eu la chance de dégager une statue qui portait la courte inscription suivante: "Lamgi-Mari2 le roi de Mari". ${ }^{3}$ Cette découverte a confirmé la proposition de W.F. Albright, qui avait autrefois conduit une prospection de surface de la région, d'identifier Tell Harīī avec l'ancienne ville de Mari. ${ }^{4}$ Même avant les fouilles de Tell Ḩarīī, Mari était déjà connue comme la ville la plus importante dans le Moyen-Euphrate, puisque d'après la tradition sumérienne elle était la dixième ville dans le dénombrement des villes sumériennes à recevoir la royauté après le déluge. ${ }^{5}$

De nombreuses saisons de fouilles ont suivi la première, et la 27ème, soit la dernière, a été menée en 1987. Les fouilles ont dégagé environ dix temples, plusieurs palais, bâtis l'un au-dessus de l'autre, des maisons d'habitation et des parties de la

\footnotetext{
1IASM.; F. Thureau-Dangin, RA XXXI (1934), p. 144.

${ }^{2}$ On lit le nom maintenant Išqi-Mari, cfr. M. Krebernik, $Z A$ LXXIV (1984), p. 164. ${ }^{3} I R S A$, p. 88, § IG2a.

${ }^{4}$ A. Parrot, Mari, une ville perdue..., Paris 1945, p. 219.

${ }^{5}$ T. Jacobsen, The Sumerian King List, Chicago 1939, p. 102, Col. V.23-32; IRSA, pp. 87-88, § IG1a; J.-R. Kupper, $R L A$ VII/5-6, pp. 382-383.
} 
muraille de la ville. ${ }^{6}$ On a fait aussi de nombreuses découvertes, parmi lesquelles des milliers de documents écrits, pour la plus grande partie en accadien. Les plus anciens datent du milieu du troisième millénaire, et les plus récents du milieu de

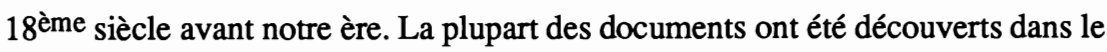
palais royal de l'époque babylonienne ancienne. Il s'agit d'un énorme palais dont la surface était d'environ 2,4 hectares, comprenant 300 chambres, cours intérieures et corridors.

Après la quatrième saison de fouilles, le nombre de tablettes et de fragments de tablettes qui avaient été trouvés dans les chambres du palais, a été estimé par A. Parrot à 20 000. Les tablettes ont été transportées de la Syrie au Louvre où elles ont été examinées pour la première fois par le grand assyriologue $F$. ThureauDangin. Il a procédé au premier classement des documents en fixant la date de la plupart des tablettes au milieu du 18 ème siècle avant notre ère - l'époque de Hammurabi, le roi de Babylonie. F. Thureau-Dangin a publié aussi, en 1936, la première lettre des archives royales (TEXT.), et a formé le noyau de l'équipe francobelge des assyriologues en confiant la publication de nombreuses lettres qui avaient été trouvées dans la chambre no. 115 à G. Dossin, qui a été pendant de nombreuses années à la tête d'une grande équipe d'assyriologues français et belges, ainsi qu'à Ch.-F. Jean. Aujourd'hui l'équipe d'assyriologues est dirigée par J.-M. Durand. Jusqu'à maintenant ont été publiées environ 1750 lettres et environ 4800 documents économiques, administratifs et juridiques. La plupart des documents ont été publiés dans la série "Archives Royales de Mari (ARM)". Dans les documents de Mari du 18 ème siècle avant notre ère un vaste tableau historique se déploie devant nos yeux. L'espace géographique qui forme le cadre où se sont déroulés les événements décrits dans les documents de Mari va de l'île de Telmun (Bahrein) dans le golfe Persique jusqu'aux îles Kaptarum (Crète) et Alašum (Chypre) dans la Méditerranée, et d'Anšan, la capitale d'Elam, et de Sušum (Suse), toutes deux en Perse, jusqu'à Kaniš (Kültepe) et Huattuša (BoghazKöi) en Asie Mineure et jusqu'à Ḩaşurā (Haçor) dans le pays d'Israël.

L'époque décrite dans ces documents est une époque riche en événements et en vicissitudes politiques, auxquels ont pris part des dizaines de grands et de petits

\footnotetext{
${ }^{6}$ Les rapports ont été publiés dans MAM, et les rapports préliminaires dans Syria et M.A.R.I.
} 
rois, des rois de grands royaumes et de petites villes-royaumes. Dans les documents se reflètent à la fois les événements de grande portée et la vie journalière de la couche gouvernante ainsi que des simples citoyens.

\section{L'histoire de la recherche sur les tribus amurrites de Mari}

Les documents de Mari contiennent entre autres une riche information sur des tribus semi-nomades, qui du point de vue ethno-linguistique sont à classer dans la branche ouest des peuples sémitiques, qualifiés d'Amurrites dans les documents de la troisième dynastie d'Ur (2112-2004 avant notre ère). La langue amurrite est un dialecte sémitique du nord-ouest, qui est proche de l'hébreu, du phénicien, de l'ugaritique et de l'araméen. On ne trouve pas de textes écrits dans la langue amurrite et elle nous est connue seulement par des mots isolés, qui étaient intégrés dans les textes accadiens, comme hamqum, ha'arum et hazzum, par des noms géographiques comme Ilānșurā, Yā'il et Tizrah, par des noms d'unités tribales comme Amurrum, Amnanûm, Bini-Yamina, Bini-Sim'āl et Yarihûum, par des noms divins comme Yakrub-El, Yatūr-Mer, et surtout par des milliers de noms propres comme Baḩli-Addu, Habdu-Dagān, Lā'ûm, Sūmu-Ilā et Samsānum. Nous identifions ces tribus comme appartenant à la couche amurrite de la population par leurs noms et les noms de la plupart des membres de ces tribus. ${ }^{7}$

G. Dossin a publié en 1938 sous le titre "Les archives épistolaires du palais de Mari" (AREP.) ${ }^{8}$ un premier rapport sur le contenu des lettres qui se trouvaient dans les archives royales de Mari. Dans cet article il s'est référé aussi aux tribus semi-nomades. Après avoir énuméré plusieurs villes et pays qui figurent dans les lettres, il écrit: "Ces villes et leurs chefs représentent l'élément sédentaire de la population, mais il a toujours existé sur les confins de la Mésopotamie un élément nomade ou demi-sédentaire. La correspondance du Palais de Mari met bien en lumière le conflit perpétuel qui met aux prises le sédentaire, qui possède, et le nomade ou le montagnard, qui n'a rien et désire posséder... Dans la Mésopotamie

\footnotetext{
${ }^{7}$ Voir J.-R. Kupper, Nomades, pp. 34, 71, 93, 227 et voir ci-dessous Chapitre III, § 6.

${ }^{8}$ Syria XIX (1938), pp. 105-126.
} 
du Nord et sur le Moyen-Euphrate, les villes doivent compter avec les Habiru, les Rabbû, les Bené-ia-mi-na et les Bené- Si-im-a-al. Les deux dernières tribus paraissent avoir joué un rôle important à cette époque, à en juger par les fréquentes mentions qu'en font les lettres". 9 Par ces mots, G. Dossin a défini le coeur du problème qui occupe les spécialistes depuis, à savoir les relations entre les sédentaires d'une part, et les nomades et les semi-nomades, d'autre part.

Un an après, en 1939, G. Dossin a publié un article qui était consacré à l'une des tribus qui apparaissent dans les documents de Mari: "Benjaminites dans les textes de Mari" (BENJ.). ${ }^{10}$ Cet article est encore une source très importante d'informations sur cette tribu, car la plupart des documents que G. Dossin a utilisés sont encore inédits. G. Dossin étudie les divers aspects de la vie de la tribu, et il faut souligner plusieurs de ses constatations qui ont eu une importance particulière dans les recherches ultérieures sur la tribu. Il voyait dans les Bini-Yamina des "bons nomades", 11 qui étaient "une de ces peuplades amurrites [qui ont envahi le "Croissant fertile"] turbulentes et pillardes en quête de butin ou de sol fertile dans la vallée du Tigre et de l'Euphrate".12 Mais en même temps, il souligne aussi le fait qu'à côté de l'élément nomade dans la tribu, on trouve un élément sédentaire: "Ces témoignages nous montrent la tribu des Benjaminites installée dans des villes et des villages échelonnés le long de l'Euphrate entre Mari, Terqa et Sagarâtum. Elle s'y adonne à la culture du blé". ${ }^{13}$ Il faut aussi souligner le fait que G. Dossin a été le premier à mettre en rapport les données de Mari sur les tribus amurrites et la Bible. Il écrit en effet: "La présence dans la région de Harrân d'une de ces tribus amurrites, les Benê-iamina, au nom identique à celui d'une tribu d'Israël...ne manquera pas d'attirer l'attention des exégètes de l'Ancien Testament, puisqu'aussi bien la Genèse signale cette même région pour avoir été une des étapes importantes de l'itinéraire suivi par Abraham et sa famille à leur sortie d'Ur en Chal-

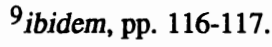

${ }^{10}$ Mélanges Syriens offerts à M. R. Dussaud, Paris 1939, pp. 981-996.

$11_{\text {ibidem, p. } 987 .}$

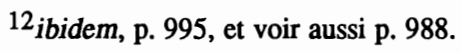

13 ibidem, p. 985.
} 
dée". 14 En effet, la question des rapports entre les tribus amurrites de Mari et l'histoire d'Israël à l'époque des Patriarches et aussi à l'époque de l'installation des Fils d'Israël en Canaan occupait dès lors une place importante dans la recherche historique et socio-anthropologique. ${ }^{15}$

En 1957, vingt années après la parution de l'article de G. Dossin sur la tribu BiniYamina a paru la grande étude de J.-R. Kupper intitulée: Les nomades en Mésopotamie au temps des rois de Mari. ${ }^{16}$ Dans son étude, J.-R. Kupper étudie le processus par lequel les nomades pénètrent dans les pays civilisés et s'y installent. Il

\section{4 ibidem, p. 996.}

${ }^{15}$ R. de Vaux, Les Patriarches Hébreux et les découvertes modernes, $R B$ LIII (1946), pp. 321348; LV (1948), pp. 321-347; LVI (1949), pp. 5-36; en particulier RA LVI, p. 15; J.-R. Kupper, Nomades, pp. XIII, 79, 81, 132; H. Klengel, Wissenschaftliche Zeitschrift der Humboldt-Universität zu Berlin VIII/2-3 (1958-1959), p. 226, et n. 220; A. Malamat, Encyclopaedia Biblica IV, cols. 559-579 (en hébreu); JAOS LXXXII (1962), pp. 143-150; CRXV $V^{e_{R A I}}$, pp. 129-138; VT S XI (1988), pp. 165-176; Mari and the Early Israelite Experience, Oxford 1989; G.E. Mendenhall, BA XXV (1962), pp. 66-87; J.T. Luke, Pastoralism and Politics in the Mari Period: A Re-examination of the Character and Political Significance of the Major West Semitic Tribal Groups of the Middle Euphrates, ca. 1828-1758 B.C., University Microfilm International, Ann Arbor, Michigan 1965, pp. 4-5; M. Weippert, Die Landnahme der israelitischen Stämme in der neueren wissenschaftlichen Diskussion, Göttingen 1967, pp. 106, 110 , 122-123; H. Klengel, $Z$ wischen Zelt und Palast, Vienne 1972, pp. 40, 181-183; T.L. Thompson, The Historicity of the Patriarchal Narrative, BZAW CXXXIII (1974), p. 197; M.B. Rowton, JNES XXXVI (1977), pp. 195-197; CRXVe $R A I$, pp. 118, 121; C.H.J. De Geus, The Tribes of Israel, Amsterdam 1976, pp. 172-174, 180-181; J.E. Mendenhall dans Magnalia Dei, The Mighty Acts of God, éd. F.M. Cross, W.E. Lemke et P.D. Miller, Garden City N.Y. 1976, p. 141; W. Thiel, Die soziale Entwicklung Israels in vorstaatlicher Zeit, Ncukirchen-Vluyn 1980, pp. 21-31; N.K. Gottwald, The Tribes of Yahweh. A Sociology of Religion of Liberated Israel 1250-1050 B.C.E., New York 1979, pp. 293, 435-436, 438, 442, 465, 545, 890; N.P. Lemche, Early Israel, VT S XXXVII, Leyde 1985, pp. 159-163. Dans ma version hébraïque du présent ouvrage j'ai traité brièvement la question dans le neuvième et dernier chapitre intitulé: "Les tribus amurrites et l'installation des Fils d'Israèl en Canaan".

${ }^{16}$ Paris 1957. 
ne se contente pas d'étudier les nomades de l'époque des archives (la première moitié du 18 ème siècle avant notre ère), mais il élargit le cadre de son étude aux nomades des époques antérieures et postérieures à l'époque des archives. Dans l'introduction à son étude, il écrit: "La pression que les nomades exercent sur la bordure du désert est donc constante. Leur avance, elle, est fonction de la résistance offerte par les cultivateurs sédentaires. Ce n'est pas, d'ordinaire, une montée subite du flot qui submerge les digues, mais les digues qui s'affaissent, mal entretenues. Ainsi les mouvements du nomadisme sont liés essentiellement aux conditions politiques, aux faits humains". ${ }^{17}$ Et dans la conclusion de son ouvrage, il écrit: "En vérité, le réservoir paraît inépuisable. Le mouvement est sans fin; les clans succèdent aux clans, les peuples aux peuples. Les 'Ouest-Sémites' ont pris la place des Martu. ${ }^{18}$ Plus d'une fois, on les a confondus, comme on confondra entre eux les groupes qui viendront sur leurs pas: Hanéens et Benjaminites, Sutéens et Aḥlamû, Aḩlamû et Araméens". ${ }^{19}$ D’après J.-R. Kupper, les documents de Mari sont importants pour l'examen de la question de l'origine des tribus amurrites, bien qu'il souligne qu'à l'époque de Mari on ne décèle pas de grands mouvements de nomades à partir des confins du désert vers le pays cultivé, car les autorités centrales sont fortes, et elles empêchent leur pénétration violente dans le royaume. Les nomades qui apparaissent dans les documents de Mari sont arrivés dans les endroits qu'ils occupent encore avant l'époque des archives. ${ }^{20}$ Les tribus ont pénétré de la Chamieh du désert syrien dans la Djéziréh, la Mésopotamie, ${ }^{21}$ les Ḩanéens se sont dirigés d'abord vers l'Idamaraș, le triangle du Habur, 22 et les Bini-Yamina, vers les steppes de la Haute-Mésopotamie, puis

\footnotetext{
${ }^{17}$ ibidem, p. XI.

${ }^{18}$ Les Martu sont les Amurrites de la troisième dynastie d'Ur. A l'époque, on pensait encore qu'il y avait une différence entre eux et les Amurrites de l'époque d'Isin-Larsa et de l'époque babylonienne ancienne.
}

19 ibidem, pp. 262-263.

20 ibidem, pp. XV-XVI, 33, 69-70, 72.

21 ibidem, p. 79.

22 ibidem, p. 37. 
ils sont descendus vers les vallées de l'Euphrate et du Habur. ${ }^{23}$ Quand les membres des tribus se sont trouvés dans le pays cultivé, ils se sont mêlés à la société locale qui est devenue de la sorte une société mixte - comprenant l'élément urbain et l'élément non-sédentaire - "les deux éléments sont complémentaires". ${ }^{24} \mathrm{~J} .-\mathrm{R}$. Kupper insiste sur la dualité de la société qui se reflète dans les archives de Mari. ${ }^{25}$ Cette notion de "dualité", sous le terme "Dimorphic Structure", sera reprise par la suite de la recherche sur les tribus. Quant à l'économie des tribus, il signale qu'elles s'occupent de l'élevage et de l'agriculture saisonnière. ${ }^{26}$ Il voit dans leur agriculture saisonnière le premier stade du processus de leur sédentarisation, qui aboutira à la fin à faire du nomade-pasteur un sédentaire-cultivateur. Mais J.R. Kupper signale que parfois la situation s'est retournée et le sédentaire-cultivateur devient de nouveau un nomade-pasteur à cause des changements politiques ou physiques. ${ }^{27}$ J.-R. Kupper classe les tribus de Mari d'après leur niveau de sédentarisation: les Sutûm sont encore des nomades, les Bini-Yamina sont à un stade transitoire de sédentarisation, et les Huanéens sont les plus proches de la sédentarisation. 28

Une année après la parution de l'ouvrage de J.-R. Kupper, H. Klengel a fini sa dissertation sur le sujet "Benjaminiten und Hanäer zur Zeit der Könige von Mari". L'ouvrage de J.-R. Kupper lui est parvenu peu de temps avant qu'il l'ait achevée, et c'est pourquoi il ne pouvait pas s'y référer. Pour cette raison il a publié en 1958 un article intitulé "Benjaminiten und Hanäer", ${ }^{29}$ dans lequel il note seulement les sujets qu'il traitait dans sa thèse et qui s'ajoutent à l'étude de J.-R. Kupper. J.-R.

23 ibidem, p. 142.

24 ibidem, p. 30.

$25_{\text {ibidem, p. } 31 .}$

26 ibidem, pp. XII-XIII.

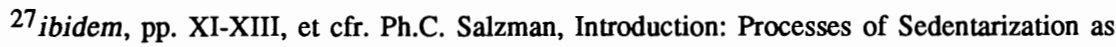
Adaptation and Response, dans When Nomads Settle, Ph.C. Salzman (éd.), [New York, 1980], pp. 1-19.

28 ibidem, pp. 55, 58, 72.

${ }^{29}$ Wissenschaftliche Zeitschrift der Humboldt-Universität zu Berlin VIII/2-3 (1958-1959), pp. 211-226. 
Kupper s'est déjà référé plusieurs fois aux ouvrages anthropologiques. ${ }^{30} \mathrm{H}$. Klengel, quant à lui, emploie assez souvent l'ouvrage de M.P. von Oppenheim Die Beduinen (première partie), ${ }^{31}$ pour éclaircir des sujets qui concernent les nomades de Mari et qui ne s'expliquent pas assez par les anciens documents. $\mathrm{H}$. Klengel décrit le lent passage des nomades à la sédentarisation partielle et puis à la sédentarisation complète, ${ }^{32}$ et il montre aussi comment les autorités essaient d'installer les nomades. 33

En 1962, A. Malamat a publié la première d'une série d'études (voir ci-dessus) où il a comparé les tribus bibliques et les tribus mariotes en ce qui concerne leurs modes d'organisation, leurs institutions sociologiques et politiques et leurs processus d'installation.

La même année, en 1962, G.E. Mendenhall a publié son article révolutionnaire sur The Hebrew Conquest of Palestine. ${ }^{34}$ Dans cet article il a lancé une nouvelle conception sur le début de l'histoire d'Israël: au lieu de parler d'une pénétration des Israélites du désert en Canaan, il suppose que la naissance d'Israël est due avant tout à une évolution à l'intérieur de la société cananéenne. Et comme Mari représente le plus ancien exemple de relations entre des tribus nomades et une société citadine, il se réfère aussi aux tribus de Mari. Il soutient qu'à Mari on a affaire à des pasteurs, qui étaient en réalité des villageois qui se spécialisaient dans l'élevage du bétail. Ils se sont tournés vers cet état en raison du manque de terres aptes à fournir de la nourriture à toute la population. ${ }^{35} \mathrm{D}$ 'après lui "Kupper...has greatly overestimated the amount of true nomadism in the Mari period. Much of the material refers simply to seasonal transhumance of sheepherding villagers". ${ }^{36}$ Il souligne qu'en dépit du fait que dans les documents de Mari il s'agit parfois de très grands troupeaux de moutons qui transhument à de longues distances en

\footnotetext{
${ }^{30}$ Nomades, pp. X-XI, XIII, XIV, n. 1.

${ }^{31}$ Leipzig 1939.

32 Benjaminiten und Hanäer, p. 217.

33 ibidem, p. 215.

${ }^{34} B A$ XXV (1962), pp. 66-87.

$35_{\text {ibidem, p. } 69 .}$

36 ibidem, p. 69 , n. 7.
} 
quête de pâturages, il n'y a pas lieu de conclure pour autant qu'il existe un contraste extrême entre la culture des éleveurs de moutons et la culture villageoise. ${ }^{37}$ Quant au lien ethnique entre les membres de la tribu, il suppose que "The tribe of Benjaminites of the Mari letters is certainly a large social complex with considerable geographical range and with at least four subsections. It is hardly likely that genealogical descent actually produced this, or any other tribe in antiquity".38

En 1965, sous la direction de G.E. Mendenhall, J.T. Luke a écrit sa thèse de doctorat intitulée "Pastoralism and Politics in the Mari Period: A Re-examination of the Character and Political Significance of the Major West Semitic Tribal Groups of the Middle Euphrates, ca. 1828-1758 B.C. ${ }^{39}$ Comme nous l'avons vu, G.E. Mendenhall a exprimé des réserves sur les conclusions de J.-R. Kupper quant au caractère nomade des tribus de Mari. J.T. Luke examine de nouveau tout le matériel de Mari concernant les tribus semi-nomades, et cela dans le but de formuler une théorie alternative à celle de J.-R. Kupper, qui étayerait la thèse de G.E. Mendenhall sur la Bible et sur Mari. Dans la conclusion de son étude, J.T. Luke écrit: "A primary objective of this study has been to challenge the theory that nomadic invasion or infiltration and subsequent sedentarization was an adequate basis for describing the relations between the tribal groups and urban society in the Mari period. As an alternative to that theory, it is proposed here that the urban-tribal relationship of the Mari period was, in fact, a complex political problem. The urban authorities sought to establish and maintain authority and constituency. To do this, they pursued various policies which affected tribal territorial claims, migration patterns, and independence. The tribal groups responded to these policies as they enhanced or threatened their interests". 40 La conclusion de J.T. Luke en ce qui concerne la relation entre la théorie de G.E. Mendenhall et la situation à Mari est la suivante: "The Mari texts may not exhibit precisely what Professor Mendenhall calls 'withdrawal', but these documents do splendidly attest the attempt of urban-political authority to extend its control over village-pastoral groups. In con-

\footnotetext{
37 ibidem, p. 69.

38 ibidem, p. 70.

${ }^{39}$ University Microfilm International, Ann Arbor, Michigan 1965.

40 ibidem, p. 243.
} 
trast to Kupper, the conclusion of this thesis will demonstrate that it was a contrast between urban interests and those of the village-pastoral groups which accounts for the areas of conflict between the West Semitic tribes of the Mari period and the urban government at Mari." 41 J.T. Luke considère l'emploi des matériaux ethnologiques comme une composante centrale dans sa méthode: "The method of the present study is interdisciplinary. The linguistic and historical data of the Mari texts will be interpreted with the aid of observations from ethnological research. Studies by ethnographers constitute a primary source of information on modern Near Eastern tribes". 42 Et il continue en disant: "Ancient sources normally yield fragmentary information. These fragments must be connected and the gaps filled before a historical reconstruction results. The reconstruction must then be viewed in historical continuum. To accomplish these tasks, the historian uses hypothesis, ideal model, or analogy in conjunction with his primary sources. " 43 Le matériel ethnographique essentiel dont J.T. Luke fait usage est, d'une part, l'ouvrage de H. Charles Tribus moutonnières du moyen Euphrate, ${ }^{44}$ qui décrit les tribus 'Agêdât, que l'auteur a visitées en 1936, et, d'autre part, l'ouvrage de M.P. von Oppenheim Die Beduinen, ${ }^{45}$ qui traite de toutes les tribus bédouines de la Syrie au début du siècle. J.T. Luke s'oppose à l'avis de J.-R. Kupper, qui a suivi en cela G. Dossin, d'après lequel une hostilité éternelle régnerait entre le nomade et le sédentaire, ${ }^{46}$ en soutenant, qu'on trouve "the high degree of interdependence of the various economic communities of the Near East". 47 Et il continue en disant: "The interdependence of the village and the nomadic groups of the Near East is the result of adaptation to natural environment". 48 J.T. Luke met en relief le facteur de transhumance saisonnière des troupeaux, qui se reflète dans les documents de Mari. Il

\footnotetext{
$41_{\text {ibidem, p. } 38 .}$

42 ibidem, p. 5.

43 ibidem, p. 6.

${ }^{44}$ Damas 1939.

45 Vol. I, Leipzig 1939.

46J.-R. Kupper, Nomades, p. XI.

47J.T. Luke, Pastoralism, p. 26.

48 ibidem, p. 27.
} 
ne s'agit pas, d'après lui, d'une pénétration des nomades dans le pays des sédentaires, ou d'un processus d'adaptation à la vie des sédentaires, mais plutôt d'un genre de vie basé sur l'agriculture et l'élevage. ${ }^{49}$ Il ne parle même pas de tribus nomades, mais de groupes de pasteurs villageois. ${ }^{50} \mathrm{Il}$ en trouve un exemple dans le genre de vie qui combine l'agriculture et l'élevage dans la confédération tribale 'Agêdât: "The economic activity of the 'Agêdât certainly does not justify a radical contrast between sedentary and pastoral groups. Their pattern of existence rather is a vivid illustration of the necessity of pastoral and sedentary interdependence. Evidence for a similar interdependence between pastoralists and sedentary groups in the ancient Near East must now be sought". 51 D'après lui, les tribus amurrites de Mari, qui habitaient dans la région du Moyen-Euphrate, ont combiné une agriculture villageoise avec l'élevage des ovins, et chaque année elles partaient pour les transhumances saisonnières dans les steppes du nord et du nord-ouest. L'économie des diverses tribus se ressemblait et on ne trouve aucune différence dans le degré de leur installation 52 : "The theory [de J.-R. Kupper] also assumes that nomads from the desert or steppe constantly wait to infiltrate or invade the sown and take possession of territory, thus creating a constant conflict with the settled population. This process is not attested at Mari". ${ }^{53}$ Au début de l'époque des archives il y a un témoignage de la présence des tribus dans la région du Moyen-Euphrate en unités politiques indépendantes, ${ }^{54}$ mais avec cela il souligne, comme J.-R. Kupper, que: "It is axiomatic to the understanding of Near Eastern nomadic groups, as related to urban political authority, that political stability tends to limit nomadic activity, while political disintegration permits mass migration, or invasion and conquest by nomads".55 J.T. Luke se réfère aussi à la pénétration amurrite en

\footnotetext{
$49_{\text {ibidem, p. } 71 .}$

$50_{\text {ibidem, p. } 37 .}$

$51_{\text {ibidem, p. } 29 .}$

52 ibidem, pp. 277-278.

53 ibidem, p. 278.

54 ibidem.

$55_{\text {ibidem, p. } 32 .}$
} 
Babylonie à la fin de l'époque de la troisième dynastie d'Ur. ${ }^{56} \mathrm{~A}$ son avis, les Amurrites n'étaient pas des nomades, mais ils ont combiné l'agriculture et l'élevage comme les tribus de Mari, et il ne faut pas non plus chercher leur origine dans la steppe syrienne, comme le pense J.-R. Kupper, mais plutôt dans les localités situées en bordure de la steppe. ${ }^{57} \mathrm{Et}$ encore: "The primary observation on these early Amorite movements indicated by the present study is that migration of this type is not necessarily synonymous with nomadism. The nomad-sedentary contrast cannot be depended upon as an explanation of movements nor the subsequent political involvement of the Amorites, leading to the rise of Amorite state". 58 Il faut signaler, qu'il dit aussi à propos des 'Agêdât: "The 'Agêdât tribes have maintained this traditional economic pattern [agriculture et élevage] for at least two centuries". 59 Mais il ne mentionne pas l'histoire d'Agêdât qui a précédé ces deux cents ans $! 60$

L'année même où J.T. Luke présentait sa thèse de doctorat à l'Université de Michigan, thèse dans laquelle il essayait d'appuyer la théorie de G.E. Mendenhall, M. Weippert présentait sa thèse de doctorat à l'Université Georg-August de Göttingen, dans laquelle il réfutait catégoriquement cette même théorie. La thèse a été publiée en 1967 sous le titre Die Landnahme der israelitischen Stämme in der neueren wissenschaftlichen Diskussion. ${ }^{61}$ Quand il discute des exemples historiques du processus d'installation des Israélites en Canaan il dit: "Die reichen Keilschrifttextfunde in der alten Königstadt Mari ... haben uns in die Lage versetzt, uns ein genaueres Bild von dem Vorgang der Niederlassung von Nomaden oder 'Halbnomaden' zu machen, die Alt der historischen Erklärung der israelitischen Landnahme zugrunde legte". ${ }^{2}$ D'après lui: "Es ist deutlich, dass sowohl Jamini-

\footnotetext{
${ }^{56}$ Voir ci-dessous Chapitre VIII.

57 ibidem, pp. 20, 279.

$58_{\text {ibidem, p. } 280 .}$

$59^{\text {ibidem, p. } 28 .}$

${ }^{60}$ Voir ci-dessous Chapitre VIII.

${ }^{61}$ Göttingen 1967. L'ouvrage a été traduit en anglais The Settlement of the Israelite Tribes in Palestine, London 1971.

62 ibidem, p. 106.
} 
ten wie Hanäer sich zur Zeit der Könige von Mari im Übergang von der nomadisierenden zur halb und ganz sesshaften Lebensweise befinden". ${ }^{63} \mathrm{Et}$ il s'oppose à l'avis de G.E. Mendenhall et J.T. Luke qui ont considéré que J.-R. Kupper avait exagéré en insistant sur l'élément nomade à Mari, et qu'à la vérité il s'agissait de nomadisme saisonnier. M. Weippert écrit: "Gewiss sehen wir neben den Schafherden der Nomaden, etwa der Jaminiten, in den Texten aus Mari auch die Herden des Königs der Stadt - und Dorfbewohner und - unter letzteren - auch die der inzwischen halb oder ganz ansässig gewordenen hanäischen Sippen und Sippenverbände. Aber es ist offenkundig, dass die Jaminiten wie die Hanäer und Sutäer von den wirklich sesshaften Leuten, den einheimischen Babyloniern wie ihrer babylonisierten 'amoritischen' Oberschicht, als schweifende barbarische Eindringlinge aus der Welt der Steppe, kurz, als Nomaden angesehen worden sind, die man nach Möglichkeit - eventuell mit staatlicher Hilfe - anzusiedeln oder aber auf bestimmte Gebiete einzuschränken oder zu vertreiben suchte. Es ist beinahe unnötig zu betonen, dass wir hier in relativer zeitlicher und räumlicher Nähe mutatis mutandis einen Prozess verfolgen können, der eine sachgemässe Analogie zu dem von Alt und seinen Schülern entworfenen Bild vom Hergang der israelitischen Landnahme darstellt. Wie die Israeliten siedeln sich die Jaminiten und ihre Verwandten zwischen den Städten an, die sie nicht erobern können oder wollen, mit denen sich aber ein differenziertes Gefüge von Beziehungen entwickelt. Wir sehen in Mari wie in Palästina die mit wechselndem Erfolg unternommenen Versuche der bodenständigen Mächte, sich die Eindringlinge untertan zu machen, wir sehen die Jaminiten von Dumtēn stolz die Fron verweigern [III.38.19-22], des Stamm Issachar hingegen um der Annehmlichkeiten des Landes willen seinen Nacken unter das Joch beugen [Gen. 49:14-15]. Dass die Jaminiten und Hanäer anders als die Israeliten am Ende sich gegen die bodenständige Bevölkerung nicht durchsetzen konnten und in ihr aufgingen, ist aus der verschieden gearteten politischen Struktur Mesopotamiens und Palästinas und bestimmten Eigentümlichkeiten Israels durchaus zu erklären. Alles in allem ist so das Urteil erlaubt, dass das von Alt ursprünglich an modernen arabischen Beispielen entwickelte Landnahmemodell den Gegebenheiten des zweiten Jahrtausends voll entspricht und dem

63 ibidem, p. 122. 
MENDENHALLschen, das, wie wir zu zeigen versucht haben, an unhaltbaren Prämissen krankt, durch die besseren Argumente überlegen ist".64

H. Klengel est revenu sur le sujet des tribus nomades de Mari dans son ouvrage intitulé $Z$ wischen Zelt und Palast. ${ }^{65}$ Dans cette étude il a élargi la discussion (à l'exemple de l'étude de J.-R. Kupper) sur le phénomène du nomadisme dans toute l'histoire de la Mésopotamie. (Il faut souligner que H. Klengel n'a pas pris connaissance encore de l'ouvrage de J.T. Luke). H. Klengel décrit ainsi, à la suite de J.-R. Kupper, la pénétration des nomades dans les pays de culture: "In den inschriftlichen Quellen lassen sich von Zeit zu Zeit verstärkte Zuwanderungen von Nomaden festellen, die in wissenchaftlichen Literatur zuweilen als 'Wellen' bezeichnet sind ... Solche 'Wellen' sind massierte Zuwanderungen von Stammesgruppen, die im Kulturland zur vollen Sesshaftigkeit übergehen und allmählich mit der älteren Bevölkerung verschmelzen. Dabei ist weniger an die Einwanderung ganzer Stämme zu denken als an Teile eines Stammes, an kleinere Gruppen ... Ob sich dieser Prozess langsam oder rasch vollzog, hing von verschiedenen Bedingungen $a b$... Neben den 'Wellen', die als ein Prozess zu verstehen sind, der sich über einen längeren Zeitraum hinziehen konnte, gab es eine ständige Infiltration ... Die Infiltration von einzelnen Nomaden, Familien oder kleinen Familienverbänden hat in den Quellen natürlich weniger Widerhall gefunden als die massierte Zuwanderung mit ihren politisch umwälzenden Ergebnissen ... 'Wellen' und Infiltration haben dazu geführt, dass sich das Bevölkerungsbild im Kulturland von Zeit zu Zeit wandelte". ${ }^{66} \mathrm{H}$. Klengel cherche à expliquer la raison de la pénétration des nomades dans le pays cultivé, en vagues ou en infiltration continue, en s'appuyant sur le matériel ethnographique qu'il utilise amplement dans le présent ouvrage. Les raisons peuvent être diverses: de faibles tribus qui sont poussées par de fortes tribus, des années de disette, des changements climatiques et des diminutions de troupeaux qui amènent avec eux des périodes de famine; accroissement naturel des nomades au delà de la capacité économique de la steppe; et enfin,

\footnotetext{
${ }^{64}$ ibidem, pp. 122-123.

${ }^{65}$ Vienne 1972.

$66_{\text {ibidem, pp. 37-38. }}$
} 
l'affaiblissement du gouvernement dans le pays cultivé ${ }^{67}$ Mais, comme J.-R. Kupper, il insiste sur le fait, que, tout comme on trouve des nomades qui deviennent sédentaires, on trouve aussi des sédentaires qui deviennent nomades à cause de raisons politiques ou de catastrophes naturelles. ${ }^{68} \mathrm{H}$. Klengel montre la symbiose qui existait dans le Moyen-Euphrate entre les nomades et les sédentaires: "Ackerbauern und Nomaden lebten in diesem Bereich am Euphrat und Habur neben- und miteinander; durch Ableiten von Flusswasser war Feldbau möglich, der gute Erträge brachte, während andererseits die nahen Weidegebiete den Herden wenigstens zeitweilig ein saftiges Futter boten. Hier war ein besonders enger Kontakt zwischen Sesshaften und Nomaden gegeben - eine Situation, die in den Texten von Mari wenigstens für einen bestimmten, kurzen Zeitraum ihre Widerspiegelung erfahren hat. Auch heute noch kann man, wenn man durch das Tal des Mittleren Euphrat reist, immer wieder zwischen den Siedlungen der Sesshaften sowie am Rande des Wüstenabbruchs die schwarzen und braunen Zelte von Nomaden sehen". ${ }^{69}$ Enfin, il faut souligner que dans la tribu il voit, contrairement à l'avis de G.E. Mendenhall, une unité ethnique: "Unter einem Stamm verstehen wir den Zusammenschluss einer Gruppe von Grossfamilien oder Sippen zu einer Einheit, die sich von einem gemeinsamen Ahnherrn ableitet".70

Durant quinze années, 1967-1981, M.B. Rowton a publié une série d'articles, destinés à constituer à la fin un seul ouvrage, où il a étudié les divers aspects du nomadisme dans le Proche Orient antique et moderne. Les titres de plusieurs de ces articles peuvent donner une idée des questions qu'il étudie: The Topological Factor in the Hapiru Problem, ${ }^{71}$ The Physical Environment and the Problem of the Nomads, ${ }^{72}$ Urban Autonomy in a Nomadic Environment, ${ }^{73}$ Autonomy and

\footnotetext{
67 ibidem, pp. 39-40.

68 ibidem, pp. 40, 219.

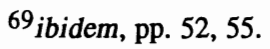

$70_{\text {ibidem, p. } 104 .}$

${ }^{71} A S$ XVI (1967), pp. 375-387.

${ }^{72} C R X V^{e_{R A I}}$, pp. 109-121.

${ }^{73}$ JNES XXXII (1973), pp. 201-215.
} 
Nomadism in Western Asia, ${ }^{74}$ Enclosed Nomadism, ${ }^{75}$ Dimorphic Structure and the Problem of the 'Apirû-'brîm, ${ }^{76}$ Dimorphic Structure and Topology, ${ }^{77} \mathrm{Di}$ morphic Structure and the Tribal Elite, 78 Dimorphic Structure and the Parasocial Element, ${ }^{79}$ Economic and Political Factors in Ancient Nomadism. ${ }^{80}$ M.B. Rowton définit ainsi le sujet de sa recherche: "The main subject under discussion in these articles is interaction between tribal society and urban society, with special emphasis on 'topology' a term used in these articles to denote the effect of physical environment on the history of a given region. I am using the term 'dimorphic structure' for the type of social and political structure which results from close interaction between tribal society and urban society, between nomad and sedentary. The kind of nomadism involved is very different from bedouin nomadism; it is termed 'enclosed nomadism' ". 81

M.B. Rowton énumère six catégories de la société dimorphique:82

"(i) The fully settled tribe which retains tribal institutions and traditions.

(ii) The semi-nomadic tribe which reverts to a nomadic mode of life for a few months each year.

(iii) A tribe, part of which is settled, part nomadic.

(iv) A region in which tribe and town, though distinct, are in close interaction.

(v) A village which pays taxes and tribute, both to a provincial town and to a nomadic tribe; undoubtedly a factor in the tendency of the peasants to desert the countryside.

${ }^{74}$ Or. XLII (1973), pp. 247-258.

75 JESHO XVII (1974), pp. 1-30.

76 JNES XXXV (1976), pp. 13-20.

${ }^{77} O A$ XV (1976), pp. 17-31.

${ }^{78}$ Festschrift J. Henninger, Studia Instituti Anthropos, XXVIII, Bonn 1976, pp. 219-257.

${ }^{79}$ JNES XXXVI (1977), pp. 181-198.

${ }^{80}$ Nomads and Sedentary People, J.S. Castillo (éd.), XXX International Congress of Human Sciences in Asia and North Africa, El Colegio de Mexico 1981, pp. 25-36.

81 JNES XXXVI, p. 181.

${ }^{82} C R X V^{e} R A I$, p. 116. 
(vi) A band composed of the socially uprooted, whether from tribe or town; so the hapirû in the Bronze Age."

M.B. Rowton définit ainsi le nomadisme enclos, qu'on trouve aussi à Mari: "enclosed nomadism involves four basic factors. First, the town in nomadic territory, highly characteristic of this form of nomadism. The town acts as a link between the nomad and the state ... Second, season migration. Because the migration goes through, or into, regions inhabited by the sedentary, it is chiefly responsible for close interaction between nomad and sedentary, tribe and state. Again, a very characteristic feature of enclosed nomadism. Third, the tendency towards symbiosis. Enclosed nomadism has its roots in a characteristic physical environment, namely in regions where pastoral land and agricultural land are closely interwoven. There is therefore a natural tendency towards symbiosis between nomad and sedentary ... The tendency towards symbiosis finds its fullest expression in the tribe which includes both full time nomads and full time sedentary ... Fourth, continuous sedentarization". 83 M.B. Rowton trouve un lien entre le déplacement saisonnier durant les mois d'été vers le pays habité et les invasions de la Mésopotamie: "When the power of the state was weak this ingressive migration could only too easily become aggressive". 84 Et encore: "... when the tribes invaded a settled areas conquest of the cities would often not have been their objective. They would be perfectly content just to secure fields and grazing land in the territory between the towns", 85 et encore: "... we have to reckon with the possibility that in those invasions conquest was only one of the factors involved. In the levels corresponding to the Gutean, Amorite, Kassite, and Aramean invasions excavation has hitherto revealed little trace of massive destruction ... For with tribesmen occupying large tracts of the countryside, with food supplies curtailed, and trade diminished, the cities would tend to shrink in upon themselves and lapse into sterile poverty". 86

\footnotetext{
${ }^{83}$ Or. XLII, pp. 257-258.

84 ibidem, p. 252.

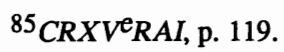

86 ibidem, p. 120.
} 
Inspiré par les études de M.B. Rowton, V.H. Matthews a écrit une thèse de doctorat, qui parut en 1978 sous le titre: Pastoral Nomadism in the Mari Kingdom (ca. 1830-1760). ${ }^{87} \mathrm{Il}$ définit son étude comme une recherche interdisciplinaire. Il y compare les sujets de Mari et un matériel anthropologique varié en faisant un vaste usage des définitions de M.B. Rowton. Il étudie des sujets tels que Definition and Character of Pastoral Nomadism, The Aspects of Herding among the Pastoral Nomads, The Social and Economic Aspects of a Symbiotic Community, The Relations between Tribe and State. Et sous l'influence de G.E. Mendenhall il parle de "la retraite" dans les documents de Mari. ${ }^{88}$

La revue de l'histoire de la recherche sur les tribus amurrites de Mari montre que deux théories ont été proposées pour expliquer l'origine des tribus amurrites et leurs relations avec les sédentaires. D'après l'avis de G. Dossin, J.-R. Kupper, H. Klengel, M. Weippert et M.B. Rowton, les tribus amurrites sont des semi-nomades, des pasteurs ayant une origine ethnique commune, qui ont pénétré de la steppe en Mésopotamie parfois en petits groupes et parfois par vagues violentes. Par contre, d'après G.E. Mendenhall et J.T. Luke, nous avons affaire à des relations entre deux éléments sociaux dans une même société: d'un côté des citadins et de l'autre, des villageois ayant une origine ethnique variée qui sont gouvernés par les citadins. Les deux théories établissent une analogie entre les tribus amurrites à Mari et les Fils d'Israël en Canaan.

Dans la présente étude nous comptons faire l'état de la question sur les tribus amurrites de Mari. Il faut se rappeler que J.-R. Kupper n'avait à sa disposition que les volumes $A R M I-V I$, J.T. Luke disposait en plus des volumes $A R M$ VII-IX, XI-XII et V.H. Matthews disposait en plus de ceux-ci des volumes $A R M \mathrm{X}$, XIII-XIV. Entre temps ont paru les volumes $A R M$ XIX, XXI-XXVI/1-2 et les annuaires M.A.R.I. I-V. De même il faut signaler les quelques 1 400-1500 textes inédits cités entièrement ou partiellement (la plupart). Nous allons étudier les divers aspects qui concernent les tribus: "L’organisation tribale", "La distribution géographique", "Les dirigeants tribaux", "L’économie des tribus", "Les relations entre les tribus et les autorités". Des sujets qui concernent les tribus comme la

\footnotetext{
${ }^{87}$ Cambridge MA 1978.

88 ibidem, p. 7.
} 
religion, les coutumes et la justice ne figurent presque pas dans les documents. Mais sur les sujets qui sont mieux documentés, l'information n'est pas complète et il existe pas mal de lacunes. Là où c'était possible nous avons essayé de combler ces lacunes par les informations tirées des ouvrages ethnographiques tels que ceux de H. Charles, ${ }^{89}$ L. Stein ${ }^{90}$ et M.P. von Oppenheim ${ }^{91}$ et les souvenirs de voyage de E. Sachau. ${ }^{92} \mathrm{Au}$ demeurant, nous avons évité d'abuser de ces sources d'information, craignant de décrire des phénomènes qui n'existaient pas à l'époque de Mari. De sorte que nous n'avons utilisé ces sources que là où les phénomènes sont attestés par les documents de Mari.

Avant de pouvoir étudier les tribus elles-mêmes, il nous faudra décrire l'arrièreplan historique dans lequel les tribus vivaient. C'est pourquoi nous commençons notre étude par un chapitre sur la chronologie de Mari, de l'époque des anciens rois jusqu'à celle de Zimri-Lim, suivi d'un chapitre sur l'histoire de Mari à l'époque des dynasties amurrites. Afin de décrire l'époque de Samsi-Addu et YasmahnAddu, nous nous servirons aussi des archives de Shemshāra, l'ancienne Sušarrā, à l'est du Tigre et au nord du Zāb inférieur, ${ }^{93}$ et de Chagar Bazar dans le triangle du Hुabur. ${ }^{94}$ Pour décrire l'époque de Zimri-Lim nous utiliserons aussi les archives de Tell al Rimah, l'ancienne ville de Qațtarā, située au sud-est du Djebel Sindjar. ${ }^{95}$ Mais avant de commencer la discussion sur tous ces sujets, il faut décrire brièvement la géographie et le climat du pays où se sont déroulés les événements

${ }^{89}$ Tribus moutonnières.

${ }^{90}$ Die Schammar-Gerba, Beduinen im Übergang vom Nomadismus zur Sesshaftigkeit, Berlin 1967.

${ }^{91}$ Die Beduinen, I.

${ }^{92}$ Reise in Syrien und Mésopotamien, Leipzig 1883.

93J. Laessøe, The Shemshăra Tablets, Copenhague 1959, et voir la liste des publications isolées dans HKL I, pp. 262-263; II, pp. 153-154 et ajouter J. Eidem Iraq XLVII (1985), pp. 83-107. ${ }^{94}$ C.J. Gadd, Iraq IV (1937), pp. 178-183, Fig. 1-2; VII (1940), pp. 22-61, Pls. I-IV; O. Loretz, AOAT I (1969), pp. 199-260.

${ }^{95}$ St. Dalley, C.B.E. Walker et J.D. Hawkins, The Old Babylonian Tablets from Tell al Rimah, British School of Archaeology in Iraq, 1976. 
décrits dans les archives de l'époque, car l'histoire de chaque pays est conditionnée par sa structure géographique.

\section{La nature du pays}

Depuis le bas du plateau de l'Arménie s'étend vers le sud une vaste steppe désertique (bādia) qui aboutit au désert de Néfoud dans la péninsule arabique. La steppe est coupée par l'Euphrate, dans lequel se déversent, de son côté gauche, les deux affluents, le Balīh et le Habur. L'Euphrate coule à une profondeur de 15 à $250 \mathrm{~m}$ au-dessous du plateau steppique et forme ainsi une vallée graduée dont la largeur varie de 2 à $17 \mathrm{~km}$. Le gradin inférieur s'appelle Ez-Zur. Les falaises de l'ouest de la vallée de l'Euphrate sont beaucoup plus hautes que celles de l'est, par contre le bord ouest de l'Euphrate est beaucoup plus étroit que celui de l'est.

L'Euphrate divise la steppe en deux contrées. La steppe occidentale est appelée Chamieh (le désert de Damas) dans la partie qui s'étend jusqu'à la ligne DamasÂna, ${ }^{96}$ et au-delà de cette ligne le plateau steppique porte le nom de Hamad, avec au centre le Djebel 'Aneiza. La partie orientale du Hamad s'appelle Wādiyān ("oueds") à cause de la rangée des oueds qui se déversent dans l'Euphrate. Entre Damas et Deïr ez-Zôr s'étend un alignement montagneux. La montagne proche de l'Euphrate, qui s'élève à une hauteur de 863 mètres, s'appelle Djebel Bichrî. Cette montagne a un lien particulier avec l'histoire des tribus amurrites. La steppe sur la rive gauche de l'Euphrate s'appelle Djéziréh ("île"), la Mésopotamie. La Djéziréh est divisée d'après un premier système ${ }^{97}$ en trois parties: (1) La Djéziréh supérieure, c'est-à-dire le plateau arménien où s'élèvent les montagnes: Tur Abdîn, à 1450 m d'altitude et Djebel Qaraja, à 1919 m d'altitude; (2) la Djéziréh moyenne, qui s'étend jusqu'à la région de Bagdad - dans sa partie septentrionale s'élèvent des deux côtés de la rivière Habur deux chaînes montagneuses: Djebel Sindjar, à 1400 m d'altitude, et Djebel Abd el-Aziz, à 920 m d'altitude; (3) la Djéziréh inférieure, c'est à dire l'Irak, la Babylonie. D'après un second système, ${ }^{98}$ la Djézi-

\footnotetext{
96V. Müller, En Syrie avec les Bédouins, Paris 1931, pp. VII, IX, n. 1.

97 ibidem, p. IX, n. 1.

98P. Sanlaville, "L'espace géographique de Mari", M.A.R.I. IV (1985), pp. 18, 20.
} 
réh est divisée en deux parties: (1) la haute Djéziréh, au nord de l'isohyète de 250 mm jusqu'au pied des montagnes, (2) la basse Djéziréh, de l'isohyète de $250 \mathrm{~mm}$ jusqu'à l'Euphrate.

Dans la steppe, le facteur qui permet des cultures non irriguées produisant des récoltes annuelles, est la quantité des précipitations et leur bonne répartition et non pas la qualité du sol. 99 Au-dessus de l'isohyète de $400 \mathrm{~mm}$, on peut pratiquer de façon assez sûre des cultures non irriguées. L'irrigation aide, mais n'est pas obligatoire. Entre l'isohyète de $400 \mathrm{~mm}$ et l'isohyète de $200 \mathrm{~mm}$ ou de $250 \mathrm{~mm}$, on peut pratiquer des cultures non irriguées, mais cela entraîne des risques, car on doit avoir des pluies chaque année et dans la saison adéquate. Dans cette région on peut obtenir une stabilité économique soit par l'irrigation, soit par une variété économique qui combine l'agriculture avec l'élevage des troupeaux. Ainsi, dans les années qui ne donnent pas de bonne récolte, on peut vivre du revenu des troupeaux. Dans cette partie de la Djéziréh se trouvent aujourd'hui de nombreux tells, qui prouvent ainsi que dans l'antiquité la région était habitée en densité. Entre l'isohyète de $200 \mathrm{~mm}$ et l'isohyète de $100 \mathrm{~mm}$ s'étend la zone semi-désertique; 100 on ne peut pas y pratiquer de cultures non irriguées, mais pendant les mois d'hiver, à partir du mois de novembre, et durant les mois du printemps, la steppe se couvre de végétation et l'eau s'accumule dans les dépressions naturelles, et elle devient ainsi apte au pâturage. Par contre, en été, à partir des mois d'avril-mai, on ne peut séjourner dans cette contrée que près des puits et dans les vallées des fleuves. Dans une région entre l'isohyète de $200 \mathrm{~mm}$ et l'isohyète de $100 \mathrm{~mm}$, on peut pratiquer des cultures irriguées dans les vallées des fleuves, et obtenir ainsi de très belles récoltes. On peut aussi pratiquer l'agriculture dans les oasis, dans les lits d'oueds, dans les cirques et dans les vallées de la steppe, qui ont absorbé assez d'eau pendant l'hiver. L'isohyète de $200 \mathrm{~mm}$ donne des oscillations beaucoup plus grandes que celles de l'isohyète de $400 \mathrm{~mm}$. Au sud de l'isohyète de $100 \mathrm{~mm}$ il n'y a pas, en général, assez de pâturages pour les troupeaux.

\footnotetext{
99J.-R. Kupper, Nomades, p. IX; M.B. Rowton, OA XV (1976), pp. 20-22.

100 J.-R. Kupper, Nomades, pp. IX-X.
} 
En résumé, on peut diviser la steppe en deux types d'après leur caractère économique:101

1. Des zones où on peut pratiquer des cultures irriguées et non irriguées - les vallées des fleuves et la plaine qui s'étend au nord du Djebel Abd el-Aziz et du Djebel Sindjar.

2. Des zones où les troupeaux peuvent paitre en hiver et au printemps - la steppe qui s'étend au sud de ces montagnes et à l'ouest de l'Euphrate.

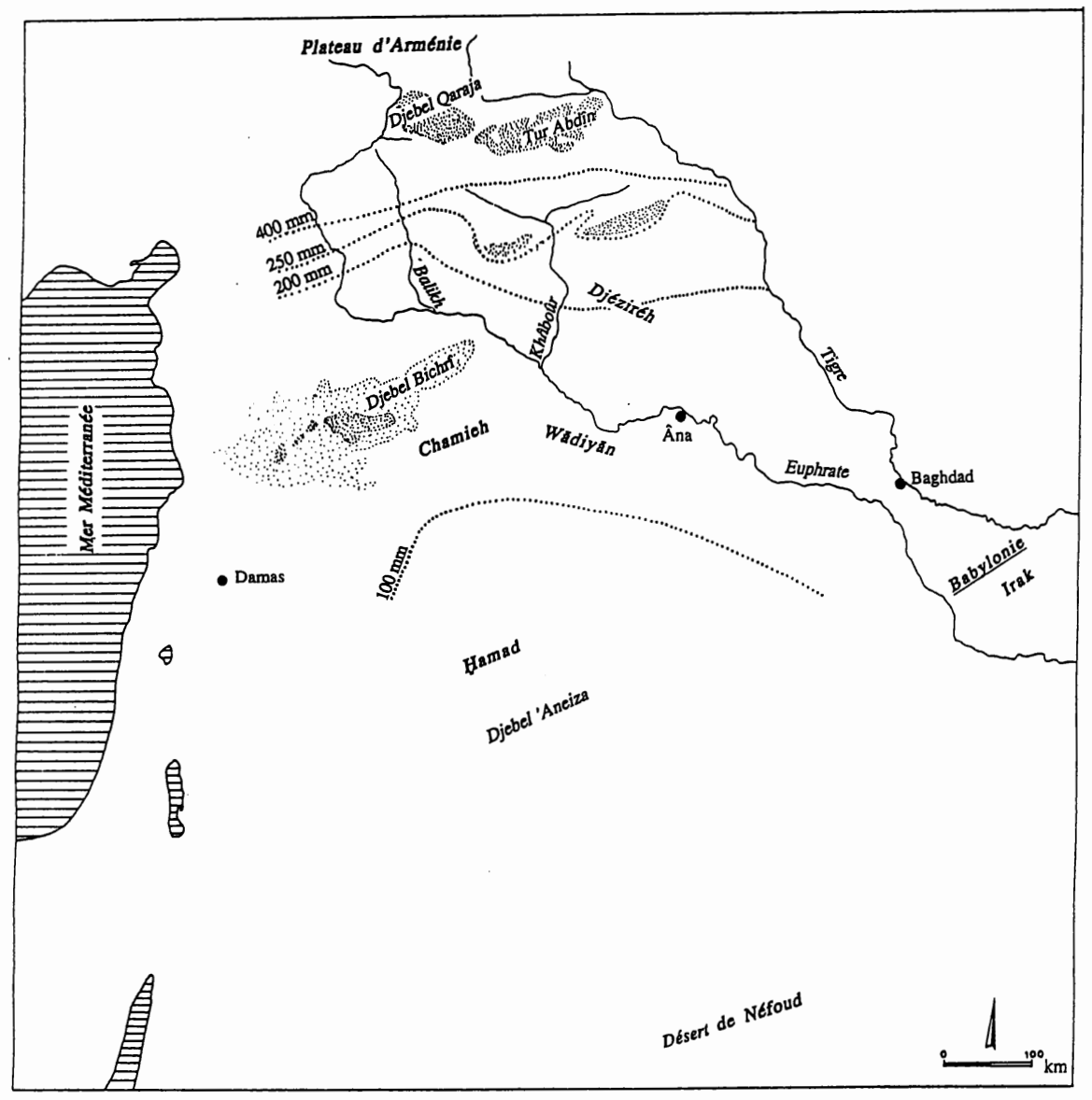

1. Une carte des régions et des précipitations

${ }^{101}$ H. Klengel, Zelt und Palast, p. 50. 


\section{Chapitre I: La chronologie de Mari}

Les documents qui ont été découverts à Mari appartiennent à trois époques: 1. L'époque des anciens rois, 2. L'époque des gouverneurs, 3. L'époque de la dynastie "Lim" et l'époque de Samsi-Addu - Yasmahn-Addu.

\section{L'époque des anciens rois}

L'époque des anciens rois correspond à celle qui va des anciennes dynasties de Sumer jusqu'à celle du royaume d'Agadé - du 27 ème siècle jusqu'au $23^{\text {ème siècle }}$ avant notre ère. Sargon, le roi d'Agadé (2334-2279 avant notre ère) a conquis Mari en 2332 avant notre ère. Cette conquête est attestée par l'un de ses noms d'années ainsi que par une inscription. Narām-Sîn, le petit-fils de Sargon (22542218 avant notre ère), a infligé une défaite à Migir-Dagan, le roi de Mari.102 Et en effet, à Mari ont été trouvés des bols portant les noms des filles de Narām-Sîn. 103 D'après la liste royale sumérienne ${ }^{104}$ au début de l'histoire de Mari, ont régné six rois.(*) D'après les documents qui ont été découverts à Ur, Mari et Ebla, nous connaissons les rois de Mari: à Ur on trouve le nom d'Ilšu, ${ }^{105}$ qui d'après la liste royale sumérienne était le premier roi de Mari. A Mari, on trouve en ordre alphabétique: Iblul-El, Ikū(n)-Šamagan, Ikū(n)-Šamaš, Išqi-Mari, Saba. ${ }^{106} \mathrm{~A}$ Ebla, on trouve en ordre chronologique - Sa'umu, Ištup-Šar, Iblul-El (qui figure aussi à Mari), Nizi, Enna-Dagan, Ikū(n-I)šar, Hiida'ar. ${ }^{107}$ A ces rois s'ajoute le roi Ikum-Mari, qui figure dans une inscription sur une jarre dont la provenance est

${ }^{102}$ E. Sollberger et A.K. Grayson, RA LXX (1976), pp. 112, 125.

${ }^{103}$ M. Lebeau, M.A.R.I. IV, p. 127; J.-M. Durand, ibidem, p. 158.

${ }^{104} \mathrm{~T}$. Jacobsen, loc. cit.

${ }^{105}$ IRSA, pp. 87-90, § IG1.

${ }^{106}$ IRSA, pp. 87-88, § IG2-6.

${ }^{107}$ A. Archi, SEb IV (1981), pp. 129-166; M.A.R.I. IV, pp. 47-51. 
inconnue. ${ }^{108}$ Il faut souligner que les vestiges des noms des cinq rois qui ont suivi Ilšu, et qui sont enregistrés dans la liste royale sumérienne (la tablette est endommagée à cet endroit) ne correspondent pas aux noms des rois de Mari connus de Mari et d'Ebla; aussi faut-il ajouter encore cinq rois au nombre des rois de Mari. En tout, nous avons affaire à dix-huit rois connus de Mari. Les documents d'Ebla permettent de dresser un synchronisme relatif avec les derniers rois d'Ebla: Nizi et Enna-Dagan étaient contemporains d'Arennum, Ikū(n-I)šar régnait au temps d'Ibrium et Hida'ar au temps d'Ibbi-Zikir. Ebla a été détruite probablement par Naräm-Sîn. ${ }^{109}$ Il faut souligner que nous ne connaissons pas les dates des règnes des rois d'Ebla, et nous ne pouvons donc pas fixer les dates exactes des règnes des rois contemporains de Mari. Nous n'avons pas de synchronisme entre les rois de Mari et les rois de Sumer, mais néanmoins il faut signaler que parmi les objets précieux qui ont été trouvés dans le "trésor" de Mari, figurait une perle fuselée en lapis-lazuli portant une courte inscription mentionnant le roi d'Ur Mesane-pada (2563-2524 avant notre ère). ${ }^{110}$

\section{L'époque des gouverneurs}

L'époque des gouverneurs šakkanakkū, qui étaient à vrai dire des rois, commence au début de l'époque d'Agadé et se termine au début de l'époque babylonienne

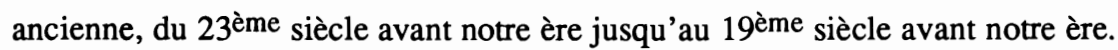
Nous connaissons les noms de 23 de ces gouverneurs-rois, ${ }^{111}$ et voici une reconstitution possible de leur ordre: Ididiš (60 ans), Šu-Dagān (5 ans), Išmah̆-Dagān (45 ans), Nūr-Mēr (5 ans), Išțup-El (11 ans), Išgum-Adad (8 ans), Apil-kēn (35

\footnotetext{
${ }^{108}$ F.M. Fales, M.A.R.I. III (1984), p. 269.

${ }^{109}$ IRSA, p. 107, § IIA4e; J. Boese, ZA LXVIII (1978), pp. 6-33.

110 IRSA, p. 42, § IB4c; J.-R. Kupper, $R L A$ VII/5-6, p. 383.

111 J.-R. Kupper, JCS XXI (1967), pp. 123-125; RA LXV (1971), pp. 113-118; IRSA, pp. 165-167, § IIIE; J.-M. Durand, RA LXXV (1981), pp. 180-181; D. Beyer, M.A.R.I. IV, pp. 173-189, 375-384; J.-M. Durand, M.A.R.I. IV, pp. 147-172; J. Margueron, M.A.R.I. IV, pp. 487-507, et voir M. Anbar, Bi Or XLIV/1-2 (1987), cols. 174-176; J.-R. Kupper, $R L A$ VII/5-6, pp. 384-385.
} 
ans; = Ur-Nammu), X (5 ans), X, ... Itūr-X, Am[er-Nunu] (6? ans), Tēr-Dagān (8 ans), Dagān-X (6 ans) ... Ili-Išar (12 ans), Turām-Dagān (20 ans), Puzur-Eštar (25 ans), Ḩitlal-Erra (7 ans), Ḩanun-Dagān (8 ans), X. Il faut intercaler dans les lacunes les deux paires suivantes: Idin-Ilum et Zinuba, Iși-Dagān et Ennin-Dagān, et les noms isolés: Idi-Dagăn, Niwar-Mēr, Laškan (?). La somme des années notées s'élève à 266 ans. Nous avons un synchronisme à cette époque entre Apilkēn, le roi de Mari, et Ur-Nammu, le fondateur de la troisième dynastie d'Ur (2112-2095 avant notre ère). ${ }^{112}$ On peut supposer que le début du règne d'Apilkën se situe dans l'espace entre 2146 et 2061 inclus. En conséquence, le début du règne d'Ididiš se situe dans l'année 2280 ou 2195 . Les derniers gouverneurs sont proches de l'époque d'Ilā-kabkabû et Yahdun-Lim dont le règne a commencé probablement en 1806/1805.

\section{L'époque de la dynastie "Lim" et l'époque de Samsi-Addu - Yasmah-Addu}

A l'époque paléobabylonienne, quatre rois ont régné à Mari: Yahdun-Lim, le fils de Yaggid-Lim, Sūmu-Yamam, le fils de Yaggid-Lim ou Yahdun-Lim, ${ }^{113}$ Yasmah̆-Addu, le fils de Samsi-Addu, et Zimri-Lim, le "fils" de Yahdun-Lim. Pendant les règnes de Yahdun-Lim, Sūmu-Yamam et Zimri-Lim chaque année du règne était nommée d'après un événement qui avait eu lieu l'année précédente, comme en Babylonie. Par contre pendant le règne de Yasmah-Addu les années portaient les noms d'importants personnages (des éponymes), comme en Assyrie. Notre discussion ira dans un ordre inverse à celui des rois, de sorte que nous commençons notre étude par Zimri-Lim.

A. Zimri-Lim - A cette époque nous possédons un synchronisme entre ZimriLim et Hammurabi, le roi de la Babylonie: 30 Hammurabi (1763/1762 avant notre ère) $=11^{\prime}$ Zimri-Lim. La date de la conquête de Mari par Hammurabi est attestée par des étiquettes de paniers à tablettes trouvées à Mari et qui sont datées du mois

${ }^{112}$ IRSA, p. 165, § IIIEIa; J.-R. Kupper, $R L A$ VII/5-6, pp. 384-385.

113 J.-R. Kupper, $R L A$ VII/5-6, p. 386. 
de Tichré de la 32ème année de Hammurabi (ETIQ.). ${ }^{114}$ En plus on a trouvé à Mari quatre tablettes, qui enregistrent des soldats babyloniens, datant de la même année. ${ }^{115}$ Cette année est en effet l'année de la conquête de Mari par Hammurabi attestée dans l'un de ses noms d'année. Le fait qu'on n'a trouvé à Mari aucun document administratif qui puisse être attribué à la première moitié de cette année montre, nous semble-t-il, que Mari fut conquise aux premiers jours de l'année. La première date de l'ère de Zimri-Lim est le quatrième jour du premier mois de l'année Bït-abišu, ${ }^{116}$ en conséquence on devrait tirer la conclusion que l'avènement de Zimri-Lim est antérieur à cette année, probablement deux ou trois mois auparavant (voir ci-dessous). Nous ne possédons pas de liste originale de l'ordre des années, mais on est parvenu à restaurer l'ordre de manière assez satisfaisante ${ }^{117}$ (la première colonne donne la date absolue, la seconde - les années de Hammurabi, la troisième - les années d'Ibal-pī-El, la quatrième - la place provisoire de l'année de Zimri-Lim, la cinquième - les abréviations des noms d'années):

Dates absolues Ḩammurabi Ibal-pi-El Zimri-Lim Noms d'années

$\begin{array}{lllll}1776 / 1775 & 17 & 4 & 0 & \text { après 11.X } \\ 1775 / 1774 & 18 & 5 & 1 & \begin{array}{l}\text { Bït-abišu et Annunītum } \\ \text { Kahat }\end{array} \\ 1774 / 1773 & 19 & 6 & & \text { Ah̆-Purattim } \\ 1773 / 1772 & 20 & 7 & 1 & \text { Bini-Yamina et Mišlăn } \\ \text { Samănum } \\ 1771 / 1771 & 21 & 8 & 2 & \begin{array}{l}\text { Ašlakkă, Addu de Halab et } \\ \text { Diritum }\end{array}\end{array}$

${ }^{114}$ D. Charpin, M.A.R.I. V (1987), pp. 661-662.

${ }^{115}$ A. Parrot, Syria XXIX (1952), pp. 198-199.

${ }^{116}$ XXII.313; D. Charpin, M.A.R.I. IV, p. 454.

${ }^{117}$ M. Birot, Syria LV (1978), pp. 333-343; M. Anbar, IOS IX (1979), pp. 1-8; J.-P. Materne, M.A.R.I. II (1983), p. 195, n. 1; K.R. Veenhof, M.A.R.I. IV, pp. 207-209; D. Charpin, M.A.R.I. IV, p. 253; M. Anbar, M.A.R.I. V, pp. 640-642; NABU 1989/18; J.-R. Kupper, $R L A$ VII/5-6, p. 389. 


$\begin{array}{lllll}1770 / 1769 & 23 & 10 & 4^{\prime} & \text { Samaš et Ḩabur } \\ 1769 / 1768 & 24 & 11 & 5^{\prime} & \text { Tébibtum } \\ 1768 / 1767 & 25 & 12 & 6^{\prime} & \text { Dür-Yahdun-Lim } \\ 1767 / 1766 & 26 & 13 & 7^{\prime} & \text { Hatta de Kakkulum } \\ 1766 / 1765 & 27 & 14 & 8^{\prime} & \text { Elam et Muballittum } \\ 1765 / 1764 & 28 & & 9^{\prime} & \text { Addu de Mahanum, Addu } \\ & & & & \text { d'Appăn et Yamhăad } \\ 1764 / 1763 & 29 & & 10^{\prime} & \text { Babylone et Qarni-Lim } \\ 1763 / 1762 & 30 & & 11^{\prime} & \text { Dagăn de Terqa } \\ 1762 / 1761 & 31 & & 12^{\prime} & \text { Ašlakkã II et Eluhtum } \\ 1761 / 1760 & 32 & & & \end{array}$

L'année commençait, comme en Babylonie, au printemps au mois d'Urahum = Nissan:

$\begin{array}{ll}\text { I. } & \text { Urahum } \\ \text { II. } & \text { Malkănum } \\ \text { III. } & \text { Lahhumum } \\ \text { IV. } & \text { Abum } \\ \text { V. } & \text { Huibirtum } \\ \text { VI. } & \text { IGI-KUR } \\ \text { VII. } & \text { Kinūnum } \\ \text { VIII. } & \text { Dagån } \\ \text { IX. } & \text { Lilliyatum } \\ \text { X. } & \text { Bèlet-biri } \\ \text { XI. } & \text { Kiskisum } \\ \text { XII. } & \text { Ebūrum }\end{array}$

Mois intercalaires: II ${ }^{\text {bis }}\left(\mathrm{ZL} \mathrm{5}^{\prime}\right)$, III ${ }^{\text {bis }}$ (ZL 5'), $\mathrm{V}^{\text {bis }}$ (ZL 5', ZL 6', ZL 10', ZL 11'), XII bis (Kahat, ZL 2') 
B. Yasmah-Addu - Samsi-Addu - La chronologie de cette époque est basée essentiellement sur les "Chroniques assyriennes" de Mari, qui donnent les noms des éponymes de l'histoire de la maison de Samsi-Addu. ${ }^{118}$ Le premier document de Yasmah-Addu date du 30ème jour du dixième mois de l'éponyme Hुāya-Malik. Le dernier document de l'époque de Yasmah-Addu date du 11 ème jour du cinquième mois, deux mois et demi avant le premier document de l'époque de ZimriLim. Voici l'ordre des éponymes (la première colonne donne la date absolue, la seconde - le numéro de l'année, la troisième - le nom de l'éponyme):

\begin{tabular}{|c|c|c|}
\hline $1793 / 1792$ & 1 & Ḩāya-Malik \\
\hline $1792 / 1791$ & 2 & Šalim-Aššur I \\
\hline $1791 / 1790$ & 3 & Salim-Aššur II \\
\hline $1790 / 1789$ & 4 & Ennam-Aššur \\
\hline $1789 / 1788$ & 5 & Sîn-muballiṭ \\
\hline $1788 / 1787$ & 6 & Rīš-Šamaš \\
\hline $1787 / 1786$ & 7 & Ibni-Addu \\
\hline $1786 / 1785$ & 8 & Aššur-imitti \\
\hline $1785 / 1784$ & 9 & Ili-ellati \\
\hline $1784 / 1783$ & 10 & Rigmānum \\
\hline $1783 / 1782$ & 11 & Ikūn-pīya \\
\hline $1782 / 1781$ & 12 & Asqudum \\
\hline $1781 / 178$ & 13 & Aššur-Malik \\
\hline $1780 / 1779$ & 14 & Awīliya \\
\hline $1779 / 1778$ & 15 & Adad-bani/Nimer-Sîn \\
\hline $1778 / 1777$ & 16 & Nimer-Sîn/Adad-bani \\
\hline $1777 / 1776$ & 17 & Tāb-şilli-Aššur \\
\hline $1776 / 1775$ & 18 & warki Țāb-şilli-Aššur \\
\hline
\end{tabular}

${ }^{118}$ M. Birot, M.A.R.I. IV, pp. 219-242. 
L'année éponymale a commencé en automne au mois de Niqmum = Eloul:

$\begin{array}{ll}\text { I. } & \text { Niqmum, IGI-KUR } \\ \text { II. } & \text { Kinūnum } \\ \text { III. } & \text { Tamḥirum, Dagān } \\ \text { IV. } & \text { Nabrûm, Lilliyatum } \\ \text { V. } & \text { Mammitum, Bēlet-biri } \\ \text { VI. } & \text { Mana, Kiskisum } \\ \text { VII. } & \text { Ayyarum, Ebūrum } \\ \text { VIII. } & \text { Addarum, Uraḥum } \\ \text { IX. } & \text { Magrānum, Malkānum } \\ \text { X. } & \text { Tamuz, Lahhumum } \\ \text { XI. } & \text { Abum } \\ \text { XII. } & \text { Tirum, Huibirtum }\end{array}$

C. Sūmu-Yamam - Sūmu-Yamam n'a régné qu'une courte période, car on ne le mentionne pas avec les autres rois de Mari: Yahdun-Lim, Samsi-Addu et ZimriLim. ${ }^{119}$ On connaît trois noms d'années de son époque: bït abišu et Halabit, Saggarātum et la deuxième année de Saggarātum. ${ }^{120}$ On peut supposer que le nom provisoire correspond à l'année de la conquête de Mari par Samsi-Addu, donc 1793/1792 avant notre ère, de sorte qu'on arrive aux dates suivantes:

$\begin{array}{lll}1795 / 1794 & 1 & \text { bit abišu et Halabit } \\ 1794 / 1793 & 2 & \text { Saggarātum } \\ 1793 / 1792 & 3 & \text { la deuxième année de Saggarātum }\end{array}$

${ }^{119}$ M. Anbar, IOS III (1973), p. 10; D. Charpin et J.-M. Durand, M.A.R.I. IV, p. 297 et n. 22. ${ }^{120}$ N.AN., pp. 52-53; J.M. Sasson, Dated Texts from Mari: A Tabulation, Aids and Research Tools in Ancient Near Eastern Studies, IV, Malibu 1980, p. 4. 
D. Yaḩdun-Lim - Nous connaissons 11 noms d'années de son époque, ${ }^{121}$ sans connaître leur ordre. Voici donc la liste des mots clés de ces noms d'années par ordre alphabétique: 1. Abattum, Bini-Yamina et Imār 2. Buzuran, 3. Ekallātum, 4. Habur, 5. H̦ên, 6. Mari et Terqa, 7. Pahududar, 8. Samsi-Addu, 9. Śamaš (MU-2-KAM), 10. Tuttul, 11. Zalpah. D'après notre reconstitution il a été remplacé par Sūmu-Yamam durant l'année 1795/1794 (avant notre ère). Il a commencé son règne au moins durant l'année 1806/1805 (avant notre ère). Yahdun-Lim figure dans le fragment S.24-3 des "Chroniques assyriennes" de Mari durant l'éponyme Atanum, l'avant-dernier éponyme figurant sur ce fragment. Le fragment qui suit, S.24-2, commence avec l'éponyme [Ennam]Aššur de l'ère de Yasmahn-Addu. ${ }^{122} \mathrm{D}$ 'après notre reconstitution l'écart entre le fragment S.24-3 et le fragment S.24-3 est de 4 éponymes: ${ }^{123}$

$\begin{array}{llll}\text { Date } & \text { Samsi-Addu } & \text { Sūmu-Yamam } & \text { Fragment } \\ 1796 / 1795 & \text { Atanum } & & \text { S.24-3 } \\ 1795 / 1794 & \text { Aššur-taklāku } & \text { bĩt abišu et Halabit } & \text { S.24-3 } \\ 1794 / 1793 & & \text { Saggarătum } & \\ 1793 / 1792 & \text { Ḩăya-Malik } & \text { la deuxième année } & \\ & & \text { de Saggarătum } & \\ 1792 / 1791 & \text { Salim-Ǎšsur I } & & \\ 1791 / 1790 & \text { Šalim-Aššur II } & & \\ 1790 / 1789 & \text { Ennam-Aššur } & & \end{array}$

Le fait qu'on n'ait pas trouvé à Mari son nom d'année bït abišs, l'année de son accession au pouvoir, n'est probablement pas dû au hasard, car Yahdun-Lim qui était originaire de Șuprum, a conquis Mari des mains de son dernier gouverneur.

\footnotetext{
${ }^{121}$ N.AN., p.52; J.M. Sasson, loc. cit.; G. Dossin, CRAIBL 1965, p. 403; D. Charpin et J.-M. Durand, M.A.R.I. IV, p. 294.

${ }^{122}$ M. Birot, M.A.R.I. IV, p. 239

${ }^{123}$ Cfr. K.R. Veenhof, M.A.R.I. IV, p. 207.
} 
E. Résumé - Voici le cadre chronologique que nous proposons pour l'histoire de Mari:

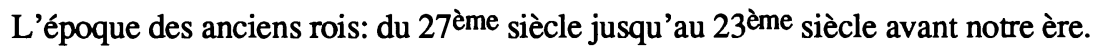

L'époque des gouverneurs: de l'année 2280 ou 2195 jusqu'à l'année 1806/1805 avant notre ère.

L'époque de la dynastie "Lim" et l'époque de Samsi-Addu - Yasmah-Addu:

$\begin{array}{ll}\text { Yahddun-Lim } & 1806 / 1805-1796 / 1795 \\ \text { Sūmu-Yamam } & 1795 / 1794-1793 / 1792 \\ \text { Yasmah-Addu } & 1793 / 1792-1776 / 1775 \\ \text { Zimri-Lim } & 1775 / 1774-1761 / 1760\end{array}$





\section{Chapitre II: L'histoire de Mari à l'époque de la dynastie "Lim" et à l'époque de Samsi-Addu - Yasmah-Addu}

\section{Yahdun-Lim (1806/1805-1796/1795 avant notre ère)}

Yaggid-Lim, le père de Yahdun-Lim, avait sa capitale à Șuprum, et sous sa domination se trouvait probablement aussi la ville de Terqa. ${ }^{124}$ Nous supposons que son royaume atteignait Dūr-Igid-Lim sur le Habur, ${ }^{125}$ l'actuel Tell Cheikh Hamed. Il est peu probable qu'il ait régné à Mari, car on n'y a trouvé aucun document datant de son époque. ${ }^{126}$ Un sceau-cylindre nous fait connaître le nom d'un de ses serviteurs, il s'agit d'Išme-El. ${ }^{127}$ Un document du temps des gouverneurs (M.5037+.3'-4') mentionne ensemble Yahdun-Lim et Yaggid-Lim. ${ }^{128}$ Tout ce que nous savons de l'histoire de son règne se trouve dans une lettre que YasmahAddu a écrite au dieu Nergal (I.3). ${ }^{129}$ D'après cette lettre, il s'avère qu'une étroite alliance existait entre Yaggid-Lim et Ilā-kabkabû, le roi d'Ekallātum ${ }^{130}$ : "Autrefois, Ilā-kabkabû et Yaggid-Lim avaient prononcé entre eux un serment solennel par le dieu". A la suite de la rupture de cette alliance, Ilā-kabkabû a détruit la forteresse de Yaggid-Lim, sans doute Șuprum, et a conquis le [pay]s(?) de Yahdun-Lim (11. 8-17). La conquête de Ṣuprum date de l'éponyme Sū-bēlī, la dernière ou l'avant-dernière année d'Ilā-kabkabû d'après les "Chroniques as-

${ }^{124}$ D. Charpin et J.-M. Durand, M.A.R.I. IV, p. 294; M. Anbar, Bi Or XLIV/1-2 (1987), col. 176.

${ }^{125} \mathrm{M}$. Anbar, IOS III, pp. 5-6.

${ }^{126}$ M. Anbar, IOS III, p. 7; D. Charpin et J.-M. Durand, loc. cit., p. 294.

${ }^{127}$ E. Weidner, AfO XVIII (1957-1958, p. 123.

${ }^{128}$ D. Charpin et J.-M. Durand, loc. cit., p. 296.

${ }^{129}$ Voir aussi M.5037+.3'-4'.

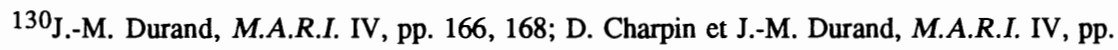
294-295. 
syriennes". ${ }^{131}$ En raison de plusieurs lacunes dans la liste éponymale, on ne peut pas fixer la date exacte de cet événement, et on doit se contenter de la date approximative de $1825 / 1824+X .{ }^{132}$ Durant l'année qui a suivi la conquête de Șuprum par Ilā-kabkabû, Samsi-Addu est monté sur le trône de son père (1824/ $1823+\mathrm{X}$ ), et a contrôlé le bord de l'Euphrate conquis par son père (I.3.F.18Rev.2').133

La ville de Mari a été conquise par Yah̆dun-Lim, le fils de Yaggid-Lim, des mains du dernier gouverneur de la ville, qui était soumise à cette époque à l'autorité d'Ešnunna. ${ }^{134} \mathrm{La}$ conquête de Mari s'est réalisée peut-être avec le soutien d'Alep (A.1968).135 (*) Nous ignorons la date exacte de la conquête, mais compte tenu du nombre de ses années attestées à Mari, on peut supposer qu'il s'agit environ de l'année 1806/1805. A la suite de la conquête, Mari est devenue la capitale de Yahdun-Lim, ${ }^{136}$ il y a transporté ses archives, où celles-ci ont été modernisées, ${ }^{137}$ et son personnel est passé aussi de Șuprum à Mari, où il s'est joint au personnel du régime précédent. ${ }^{138} \mathrm{La}$ conquête de Mari par Yaḩdun-Lim marque le début de l'écriture dans le dialecte babylonien ancien et l'apparition de l'élément typique des noms propres amurrites Ya-, absent à l'époque des gouver-

${ }^{131}$ M. Birot, M.A.R.I. IV, pp. 222, 229 .

${ }^{132} \mathrm{De}$ l'éponyme Šu-bēli jusqu'au dernier éponyme qui figure sur la tablette S.24-1+S.24-2+ A.1614b, avant la cassure, nous comptons 24 éponymes. Après une lacune la liste continue dans la tablette A.1288 avec trois éponymes, puis encore une lacune, et la liste continue avec la tablette S.24-3 avec 4 éponymes, et puis encore une lacune, qui d'après notre calcul (ci-dessus Chapitre I) compte 4 éponymes suivis de la tablette S.24-2 qui commence avec l'éponyme [Ennam]-Aššur. Si nous acceptons que la tablette S.24-3 commence en 1798/1797, la conquête de Şuprum date de l'année: $1798 / 1797+3+24+\mathrm{X}_{1}+\mathrm{X}_{2}$. Comme nous ne connaissons pas l'importance des lacunes, nous pouvons seulement affirmer que la date est 1825/1824 + X.

${ }^{133}$ M. Anbar, Bi Or XLIV/1-2 (1987), col. 177.

${ }^{134}$ D. Charpin et J.-M. Durand, M.A.R.I. IV, p. 294.

135 ibidem, p. 297.

136J.-M. Durand, M.A.R.I. IV, p. 166; D. Charpin et J.-M. Durand, M.A.R.I. IV, p. 294.

${ }^{137}$ D. Charpin et J.-M. Durand, ibidem.

138J.-M. Durand, ibidem, pp. 160, 168. 
neurs. ${ }^{139}$ Yahdun-Lim a fait de Mari la capitale d'un vaste royaume. En effet, ses noms d'années nous font savoir qu'il a conquis des villes dans l'Euphrate supérieur: Tuttul (l'actuelle Tell Bîaâ), Abattum, à mi-chemin entre Tuttul et Imār (l'actuelle Eski-Meskéné), et Imār elle-même; la ville du Balīh, Zalpah̆, et la ville de Pahudar, à l'est de Harrān. ${ }^{140}$ Une lettre d'un vassal de Yahdun-Lim montre que l'autorité de Yahdun-Lim comprenait une vaste région en face des royaumes de Hुaššum (peut-être Araban ${ }^{141}$ ), Ursum (peut-être Gaziantep ${ }^{142}$ ), Karkamiš et Yamhad, dont la capitale était Halab, (I.1.1'-9'). Il s'avère qu'il dominait aussi l'Idamaraş, ou au moins sa partie occidentale. Son représentant siégeait dans la ville de Talhāyum (XIII.143.5-9), et les textes économiques attestent une présence mariote dans les villes de Kahat (l'actuelle Tell Barri; XXII.227 et cfr. 277-278), de Șubat-Ištar (XXII.272-273) et de Tarnip (XXII.123, 161-162). ${ }^{143}$ C'est à Kahat que Yaḩdun-Lim a envoyé ses troupeaux en pacage. ${ }^{144}$ Dans son inscription de fondation, Yahdun-Lim raconte qu'il était le premier parmi les rois de Mari à parvenir à la Méditerranée, qu'il a soumis à son autorité le pays au bord de la mer, qu'il a apporté des bois des "Montagnes de Cèdres et de Buis" (M.2802.I.28-III.2). Durant l'année de cette expédition, a eu lieu le grand conflit entre Yahdun-Lim et les tribus amurrites de la vallée de l'Euphrate dont nous parlerons plus amplement dans les chapitres sur les tribus. Yahdun-Lim était aussi un roi bâtisseur. ${ }^{145}$ Il a bâti la ville de Dūr-Yaḥdun-Lim, ${ }^{146}$ et a creusé un canal pour l'alimenter en eau. ${ }^{147}$ Il a creusé encore deux autres canaux, 148 il a bâti le

${ }^{139}$ J.-M. Durand, ibidem, pp. 161, 170-171.

${ }^{140} \mathrm{~K} . \mathrm{R}$. Veenhof, Aspect of Old Assyrian Trade and its Terminology, Leyde 1972, p. 241, et voir aussi XXII.160 (cfr. J-R. Kupper, M.A.R.I. III, p. 183).

${ }^{141}$ A. Archi, P.E. Pecorella et M. Salvini, Gaziantep e la sua regione, Rome 1971, p. 44.

142 ibidem, p. 45.

${ }^{143}$ Voir J.-R. Kupper, ibidem et D. Charpin et J.-M. Durand, RA LXXXI (1987), p. 138. (*)

${ }^{144}$ D. Charpin et J.-M. Durand, M.A.R.I. IV, p. 332, et voir ausi AREP., p. 109. (*)

145J.-R. Kupper, RLA VII/5-6, p. 385.

${ }^{146}$ Cfr. J.D. Safren, JESHO XXXII (1989), pp. 1-47.

${ }^{147}$ IAHD. 35-49.

${ }^{148}$ G. Dossin, CRAIBL 1965, p. 403. 
temple de Samaš ${ }^{149}$ et il a fortifié Mari et Terqa. ${ }^{150}$ Sur ses relations économiques extérieures nous n'avons qu'un indice, à savoir l'empreinte trouvée en Asie mineure du sceau-cylindre de sa fille ${ }^{151}$ Nous pensons que pendant une certaine époque, une alliance existait entre Yahdun-Lim et Samsi-Addu (I.3.1'-2'). ${ }^{152}$ Yahddun-Lim a même rendu visite à son allié dans sa capitale Ekallātum. ${ }^{153}$ Puis Yahdun-Lim a rompu l'alliance, et une guerre a éclaté entre les deux à Nagar, ${ }^{154}$

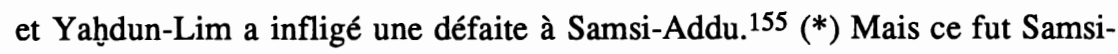
Addu qui remporta la victoire décisive (M.5037.5'-6'), ${ }^{156}$ peut-être durant l'éponymat d'Atanum. ${ }^{157}$ Dans cette guerre, Yahdun-Lim faisait partie d'une coalition de 12 alliés. On pourrait comprendre d'après la lettre de Yasmah-Addu qu'à la suite de la défaite de Yahdun-Lim, Sūmu-Yamam, son fils, l'a chassé (?) de Mari (I.3.Rev.4'-5').

\section{Sūmu-Yamam (1795/1794-1793/1792)}

D'après ses noms d'années il a bâti la ville de Halabit, proche de Tuttul du Balîh, et Saggarātum, près de l'embouchure du Habur. ${ }^{158}$ Les documents de son époque nous apprennent que parmi ses fonctionnaires se trouvaient ceux qui servaient aussi sous Yahdun-Lim, ${ }^{159}$ et ils nous font connaître des membres de sa famille

${ }^{149}$ VIII.61.4'-6'; M.2802.IV.5-14.

${ }^{150}$ IAHD.31-34; G. Dossin, RA LXIV (1970), p 166, n. 2.

${ }^{151}$ Cfr. D. Charpin et J.-M. Durand, RA LXXX (1986), p. 152.

${ }^{152}$ M. Anbar, Bi Or XLIV/1-2 (1987), col. 177.

${ }^{153}$ D. Charpin et J.-M. Durand, M.A.R.I. IV, p. 294.

${ }^{154}$ A localiser entre Kahat et Qattunăn, cfr. M. Anbar, IOS III, p. 14.

${ }^{155}$ M. Anbar, ibidem; K.R. Veenhof, M.A.R.I. IV, p. 207; D. Charpin et J.-M. Durand, M.A.R.I. IV, p. 295.

${ }^{156}$ D. Charpin et J.-M. Durand, M.A.R.I. IV, pp. 295-296, p. 297, n. 19, 1.3'.

${ }^{157}$ K.R. Veenhof, M.A.R.I. IV, p. 207; D. Charpin et J.-M. Durand, M.A.R.I. IV, p. 295.

${ }^{158}$ N.AN., p. 52 . Pour la localisation de Saggarătum cfr. notre remarque dans BiOr XLIV/1-2 (1987), col. 183, et XXVI.158.5-10. $\left(^{*}\right)$

${ }^{159}$ D. Charpin et J.-M. Durand, M.A.R.I. IV, p. 298. 
(A.4634). ${ }^{160}$ Sur ses relations extérieures nous savons seulement qu'il maintenait des relations avec le Yamhad (XXII.269). Tout ce qu'on sait de son histoire se trouve dans une lettre de Yasmah-Addu (I.3). On peut en conclure que SümuYamam était un vassal de Samsi-Addu, puis qu'il s'est insurgé contre son suzerain, et enfin qu'une révolte éclata au palais durant laquelle il fut assassiné par ses propres serviteurs ${ }^{161}$ et Samsi-Addu s'empara à nouveau du bord de l'Euphrate (I.3.Rev.6'-15'). ${ }^{162}$ La ville de Mari fut conquise par Samsi-Addu durant l'éponymat de Ḩāya-Malik, 163 et Samsi-Addu y installa son fils YasmahAddu (I.3.Rev.18'-19'). La conquête de la ville n'entraîna pas sa destruction, par contre on voit que les fonctionnaires de l'ère de Sūmu-Yamam gardèrent leurs fonctions et ils se chargèrent même du transfert du trésor du palais de Mari à la ville d'Aššur. ${ }^{164}$ Il est intéressant de noter que le transfert du pouvoir n'a pas entraîné un changement dans la hiérarchie du harem, ${ }^{165}$ et que Yasmah-Addu a joint à son harem "une bonne partie des princesses capturées au moment de la prise de Mari"..166

\section{Yasmab-Addu (1793/1792-1776/1775)}

Les règnes de Yasmah-Addu et de Zimri-Lim sont amplement documentés par les archives de Mari. Toutefois, la majeure partie des événements décrits dans les lettres ne sont pas datés, de sorte qu'il est presque impossible de les placer en ordre chronologique. Néanmoins, les historiens sont parvenus, grâce à toutes sortes de recoupements, à tresser des toiles historiques de ces deux époques. Dans

\footnotetext{
${ }^{160}$ D. Charpin et J.-M. Durand, M.A.R.I. IV, p. 298; J.-M. Durand, ibidem, p. 431.

${ }^{161}$ M. Anbar, IOS III, p. 17; D. Charpin et J.-M. Durand, loc. cit, J.-R. Kupper, RLA VII/5-6, p. 386.

162M. Anbar, Bi Or XLIV/1-2 (1987), col. 177.

${ }^{163}$ D. Charpin et J.-M. Durand, ibidem.

${ }^{164}$ D. Charpin, M.A.R.I. II (1983), pp. 211-214.

${ }^{165}$ D. Charpin et J.-M. Durand, ibidem, p. 304.

166J.-M. Durand, Le système palatial en Orient, en Grèce et à Rome, éd. E. Lévy, Strasbourg 1987, p. 85.
} 
notre description historique nous nous sommes presque toujours limités aux déroulements historiques dont on connaît, ou du moins dont on peut reconstituer, le cadre chronologique.

L'année Ḥāya-Malik (1793/1792): Cette année n'est pas conservée dans les "Chroniques assyriennes". Par contre, la conquête de Mari par Samsi-Addu est mentionnée dans l'une de ses inscriptions: "Lorsqu'Itūr-Mēr, mon seigneur, m'eût donné sans réserve le pays de Mari et les bords de l'Euphrate pour (les) posséder et (les) gouverner" (A.2231.4-9). L'installation de son fils YasmahAddu sur le trône de Mari est rappelée par ce dernier dans un passage endommagé: "[Tu a ren]du [aux mains (de Samsi-Addu) la ville de Mari] et les bords de l'Euphrate. Alors, il me prit et m'installa [...] de Mari" (I.3.16'-18'). Le premier document de la nouvelle ère date du 30.X. Les documents attestent la présence du roi (probablement Yasmah-Addu) à Mari le 6.XI et le 8.XII. Toute la documentation de Mari durant les trois derniers mois de l'année s'occupe de l'inventaire des trésors du palais de Mari et du transfert d'une partie à Aššur qui se trouvait sous la responsabilité de deux anciens fonctionnaires du temps de Yahdun-Lim et de Sūmu-Yamam. ${ }^{167}$

Les deux années suivantes, nommées d'après les éponymes Salim-Aššur I et Šlim-Aššur II (1792/1790), ne sont pas elles non plus conservées dans les "Chroniques assyriennes", en outre, les 6 documents économiques datés d'après ces éponymes ne fournissent pas de renseignements historiques. Quant aux années qui les suivent Ennam-Aššur, Sîn-muballiț, Rīš-Šamaš et Ibni-Addu (1790/1786), les "Chroniques assyriennes" n'ont conservé que quelques mots tels que: "Samsi-Addu", "pays", "Išme-Dagān" suivi de "victoire" (de l'ennemi d'Išme-Dagān). Les 13 documents de cette époque ne fournissent pas de renseignements historiques. ${ }^{168}$

\footnotetext{
${ }^{167}$ D. Charpin, M.A.R.I. II (1983), pp. 211-214; M.A.R.I. IV , pp. 256-257.

${ }^{168}$ D. Charpin, M.A.R.I. IV, p. 257.
} 
L'année Aššur-imitti (1786/1785): Les "Chroniques assyriennes" ont conservé les mots clés suivants: "Samsi-Addu", "victoire" (de l'ennemi de SamsiAddu), "pays" par deux fois, "Me-Turan" (dans la région de la Diyāla) et "Dādūša", le roi d'Ešnunna. Ces bribes d'informations montrent qu'il s'agit d'une campagne de Samsi-Addu à la frontière sud-est de son royaume, dans la région de la Diyāla, peut-être en collaboration avec Dādūša. 169

Pour les deux années suivantes Ili-ellati et Rigmānum (1784/1783), les libellés conservent seulement les noms d'éponymes. Les 8 documents de l'époque ne fournissent pas de renseignements historiques. 170

L'année Ikūn-pīya (1783-1782):171 Pour cette année, nous ne lisons que l'énoncé suivant: "Muna-[... victoire ... et Samsi-Addu ... Me-Turan ... à Dādūša ... ". Le même nom propre, Muna-[, apparaît, peut-être, aussi pendant l'année précédente, année de l'éponyme Rigmānum (1784/1783): Mu-[ . .172 Trois ans auparavant, pendant l'année de l'éponyme Ǎšur-imitti (1786-1785), on trouve aussi comme nous l'avons vu: "Samsi-Addu", "victoire", "Me-Turan" et "Dādūša". Nous ne savons pas dans quelle partie de l'année se situent les événements mentionnés dans les "Chroniques assyriennes". D'autre part, nous pouvons situer plusieurs événements qui concernent Yasmah-Addu grâce aux textes économiques. Yasmahn-Addu se déplace de 24.VIII jusqu'au 15.IX, il va de Mari, via Șuprum, à Terqa et puis rentre dans sa capitale. Ces déplacements sont sans doute liés à l'arrivée de Bēltum, la princesse de Qatanum, au 6.IX pour se marier avec Yasmah-Addu et ainsi devenir la reine de Mari. ${ }^{172}\left({ }^{*}\right)$ Quatre lettres traitent du

${ }^{169}$ M. Birot, M.A.R.I. IV, p. 226; J. Eidem, Iraq XLVII (1985), p. 88.

${ }^{170}$ D. Charpin, M.A.R.I. IV, pp. 257-258.

${ }^{171} \mathrm{La}$ description des événements durant les années éponymales suivantes est basée sur notre étude "La fin du règne de Samsi-Addu 1 er", "Reflets des Deux Fleuves", Louvain 1989, pp. 713.

${ }^{172}$ D. Charpin et J.-M. Durand (M.A.R.I. IV, pp. 311-312) proposeraient de restituer le nom Munawwirum, qui serait le nom d'un général assyrien.

172 B. Lafont, Miscellanea Babylonica, Paris 1985, p. 167. 
mariage de Yasmah-Addu avec Bēltum (I.24+M.5012; I.46; 54; 77). D'après la lettre I.24+ Sūmu-epuh, le roi de Halab razzie le pays d'Išhi-Addu, le roi de Qatanum (cfr. IV.10). Išḩi-Addu demande de l'aide à Samsi-Addu. Ce dernier répond qu'il doit d'abord atteindre son but, dans un ou deux mois. Ce "but" pourrait être une victoire dans la région de la Diyāla à laquelle font allusion les "Chroniques assyriennes", d'où la conclusion que les exploits dans la région de la Diyāla avaient eu lieu aux alentours du gème mois. On mentionne aussi une première montée de troupes d'Ešnunna, puis la montée de toute l'armée d'Ešnunna. Compte tenu des "Chroniques assyriennes", on peut supposer que SamsiAddu espère pouvoir atteindre son but grâce aux Ešnunnéens (cfr. I.42.39-41). Samsi-Addu va envoyer à Išhi-Addu une armée une fois qu'il aura atteint son but (cfr. aussi I.23; II.5). Pour le moment, il écrit à Sîn-tiri de rassembler les Hanéens et de piller les troupeaux de Sūmu-epuh et des Rabbéens. En outre, on apprend que Haššum, Ursum et Karkamiš forment une coalition avec Samsi-Addu contre Sūmu-epuh. Cette alliance date peut-être encore de l'époque du dernier conflit entre Samsi-Addu et Yahdun-Lim (I.1.4'-9'). Nous ne savons pas si Samsi-Addu est enfin arrivé chez son allié Išhi-Addu, car dans la lettre V.17+A.1882.46-47 il est dit que Samsi-Addu n'a pas envoyé l'armée. Pourtant un retour d'une armée de Qatanum est mentionné dans la lettre I.20.6'-7'.

L'année Asqudum (1782/1781): Dans les "Chroniques assyriennes" n'est conservé de cette année que le nom de Samsi-Addu. La description, datée, du déroulement des événements de cette année est basée sur l'inscription de la stèle de Samsi-Addu. ${ }^{173}$ D'après cette stèle, Samsi-Addu a conquis Arraphum au VIIİ̀me mois (col. I - col. II.11), le 20.VIII Samsi-Addu a traversé le Zāb (inférieur;col. II.12-15), a fait une razzia dans le pays de Qabrā et a détruit sa moisson (col. III.1-6). ${ }^{174}$ Au IXème mois, Samsi-Addu a conquis toutes les villes fortifiées d'Urbēl (l'Erbil moderne; col. III.7-14), et la ville de Qabrā reste seule (col. III.15 - col. IV.2). C'est probablement pendant le $X^{\grave{e ̀ m e}}$ mois que Samsi-

\footnotetext{
${ }^{173}$ H. Genouillac, $R A$ VII (1910), pp. 151-156.

${ }^{174}$ Cfr. SH.811.23-24 et SH.818.5-12.
} 
Addu l'a conquise (col. IV.6-10). Par contre d'après la stèle de Dādūša, ${ }^{175}$ c'est lui, Dādūša, qui a conquis toutes les villes d'Urbēl, capitale de Bunu-Ištar, puis c'est encore lui qui a conquis Qabrā et l'a octroyée à "Samsi-Addu, le roi d'Ekallātum". Le fait que deux rois réclament pour eux la même victoire ne doit pas nous étonner, la réalité historique qui se cache derrière ces revendications est, probablement, un exploit commun aux deux rois. ${ }^{176}$ La coopération entre SamsiAddu et Dādūša pendant ces années est suggérée par les deux "à Dādūša" qui apparaissent l'année précédente et l'année suivante dans les "Chroniques assyriennes". Cette coopération est attestée aussi par la lettre SH.882.8-10. D'après la lettre SH.827 Samsi-Addu, après avoir conquis la ville d'Arraphum, s'est dirigé sur Qabrā et a envoyé son fils Išme-Dagān assiéger Nurrugum (11. 814). Cette lettre donne l'impression que les événements sont consécutifs, mais en vérité il faut séparer la campagne d'Arraphum et de Qabrā de celles de Nurrugum et d'Ahazum. Le siège de Nurrugum qui dura jusqu'à l'année éponymale suivante a commencé peut-être à ce moment-là. ${ }^{177}$ La conquête des villes de Qabrā par Samsi-Addu et sa marche sur Qabrā sont relatées aussi dans deux lettres d'IšmeDagān à Yasmah-Addu, I.121 et IV.49 (cfr. aussi SH.861.14-15). Dans la lettre SH.809, il existe un lien entre la conquête d'Arrapha et la conquête des villes de Qabrā: Yašūb-Addu, le roi d'Ahazum, a prêté serment à Samsi-Addu à Arrapḩa puis à A'innum, l'une des villes de Qabrā (11. 19-22). La lettre I.135 (cfr. A.4413), elle aussi, raconte la conquête des villes de Qabrā, mais par Išme-Dagān. Dans les lettres I.131 et 138, c'est Išme-Dagān qui a conquis les villes de BunuIštar, le roi d'Urbēl. La lettre I.92, quant à elle, mentionne la défaite de Qabrā, suivie de la défaite de la tribu amurrite Ya'ilānum (cfr. aussi IV.33, SH.827.29 et le "nom d'année" dans VIII. 11). Si la lettre I.8, qui date du 15.XII, est en rapport avec la guerre contre les Ya'ilānéens nous obtenons la date de cette guerre. ${ }^{178} \mathrm{La}$

\footnotetext{
175 B.Kh. Ismail, Im Bannkreis des Alten Orients, Mélanges K. Oberhuber, Innsbruck 1986, pp. 105-108.

176J. Eidem, Iraq XLVII, p. 88.

${ }^{177}$ Cfr. J. Eidem, Iraq XLVII, p. 99.

${ }^{178}$ Cfr. J. Eidem, Iraq XLVII, pp. 85, 97; D. Charpin et J.-M. Durand, M.A.R.I. IV, pp. 314, n. 94 .
} 
lettre IV.25 nous prouve qu'Išme-Dagān domine les pays d'Arrapḥum et de Qabrā (11. 20-23). Une indication intéressante se trouve dans la lettre SH.878.24: Samsi-Addu (le seigneur de Kuwari) va arriver à Qabrā, puis à la fin du mois il sera déjà à Subat-Enlil, 11. 15-17. Il pourrait s'agir de la fin du Xème mois.(*)

L'année Aššur-Malik (1781/1780): Les "Chroniques assyriennes" donnent pour cette année plus d'informations que pour les années précédentes. On y lit: "Išme-Dagān victoire ... et Samsi-Addu Nur[rugum ... Kibrum, le roi de ... , Yašūb-Addu, le roi d'[Aḩazum], Yašǔb-Lim, le roi de ... , ces 9(?) rois il les a captu[rés ... à Dādūša ...". Ainsi que nous l'avons déjà vu d'après la lettre SH. 827 , on peut conclure que l'année précédente Išme-Dagān a commencé le siège de Nurrugum (11. 12-14). Ici et dans les "Chroniques assyriennes" il s'agit sans doute de la Nurrugum orientale sise à l'est du Tigre, ${ }^{179}$ vraisemblablement vers le nord du Zāb inférieur. La conquête de cette ville a été signalée par Samsi-Addu comme un acte essentiel de son règne. ${ }^{180} \mathrm{La}$ campagne de Nurrugum est mentionnée aussi dans les lettres S.115, no. 72-2 et I.22.10. On voit d'après les "Chroniques assyriennes" que la victoire sur Nurrugum est liée à une victoire sur plusieurs rois, parmi eux Yašūb-Addu, le roi d'Ahazum. Ce lien entre la campagne de Nurrugum et la campagne d'Ahazum se trouve aussi dans les lettres SH.888, SH.913, SH.915 et SH.917. Dans la lettre 1.69+M.7538, nous lisons qu'Išme-Dagān a pris la tête des troupes qui se sont rassemblées à Qabrā pour une expédition contre le pays d'Ahazum et qu'il a conquis tout le pays d'Aḩazum. Samsi-Addu, quant à lui, est parti pour "la ville", Ninive en occurrence. La lettre I.69+ contient à la fin un synchronisme important. Samsi-Addu encourage Yasmahn-Addu à aller à la tête de ses troupes à Qatanum (11. 38-43). Une série de lettres traite de la montée de Yasmah-Addu à Qatanum. Dans II. 51, Išḩi-Addu demande à Yasmah-Addu de prendre avec lui sa fille lors de son voyage. Dans les lettres I.85+A.1195 et I.42.6-7, 45 il est aussi question de l'expédition de Yasmah̆-Addu. ${ }^{181}$ Enfin, dans la lettre V.16 Išḩi-Addu écrit à Yasmah-Addu, qui

\footnotetext{
${ }^{179}$ Pour la Nurrugum occidentale cfr. XVI/1, p. 25.

${ }^{180}$ Voir R. Borger, EAK 1, p. 9, col. I.17.

181J. Eidem, Iraq XIVII, p. 101; J.-M. Durand, M.A.R.I. V (1987), p. 160.
} 
va partir pour Qatanum, qu'il se réjouit des nouvelles d'Išme-Dagān. Il s'agit sans doute de ses victoires sur Nurrugum et Ahazum (cfr. I. 69+). Des lettres inédites nous informent que "le corps expéditionnaire arriva bien à destination et eut plusieurs réussites à son actif". ${ }^{182} \mathrm{La}$ dernière date qui atteste la présence de Yasmaḩ-Addu à Mari est le 9.VII. Le 8.VII Samsi-Addu envoie une lettre à Yasmah-Addu depuis Ninive lui ordonnant d'aller à Subat-Samaš (I.60). (*) Un mois après, le 12.VIII, Samsi-Addu se trouve dans "la ville" négociant avec Ešnunna (cfr. aussi I.50 du même mois), et demande à Yasmah-Addu de ne pas aller à Qattunān pour attendre son arrivée à Subat-Enlil, mais d'aller au "coeur du pays", à Haššum-de-Membida pour purifier les Hanéens (I.37; cfr. II.1.10-28). Dans trois lettres qui précèdent la lettre I.37, Samsi-Addu écrit qu'il s'apprête à arriver à Subat-Enlil à la fin du mois (I.82.19; 87.15; SH.878.15), il s'agit probablement de la fin du Xème mois. Le voyage de Samsi-Addu a été retardé à cause des négociations avec Ešnunna. La présence de Yasmah-Addu à SubatSamaš le 6.X est attestée par un document économique (no. 102183). Deux lettres, non datées, attestent aussi la présence de Yasmah-Addu à Šbat-Samaš (I.97; X.107). Pendant le $X^{\text {ème }}$ mois Samsi-Addu voyage de Ninive à Subat-Enlil (I.10.9'-10') et se prépare à partir pour Zalmaqum, la zone montagneuse au nord de la ligne Harrān-Nisibin (1. 7'). Deux lettres sont antérieures à cette lettre: I.22 et I.29. La lettre I.22 fait allusion à la défaite de Nurrugum (11. 10-11), elle date probablement du Xème mois. Le 6, le 8 et le 13 du XIème mois, Samsi-Addu se trouve encore à Subat-Enlil (I. 67; I.5; I.19+M.9541). ${ }^{184}$ Le 30.XI, Samsi-Addu envoie une lettre à Yasmah̆-Addu depuis Subat-Enlil lui annonçant qu'il arrivera chez lui le 15.XII (II. 8.17-18). Il lui écrit aussi à propos d'Išme-Dagān qui va sans doute se joindre à Yasmah-Addu. J.-M. Durand place ici l'invasion de la

182J.-M. Durand, ibidem.

${ }^{183}$ Les textes économiques précédés de "no." sont cités d'après l'article de D. Charpin dans M.A.R.I. III (1984), pp. 83-126.

${ }^{184}$ Ici doit être intercalée, pensons-nous, la lettre XXVI.266. 
vallée de l'Euphrate par Dāduša et la seconde reconquête de Rapiqum (la première conquête est antérieure à l'éponyme Aššur-Malik). ${ }^{185}$

L'année Awīliya (1780/1779): Dans les "Chroniques assyriennes" est conservé seulement le nom de la tribu hurrite Turukkum. Le 25 et le 26 du Ier mois, on constate qu'Išme-Dagān s'est joint à Yasmah̆-Addu (II.10; I.39; IV.28 [cfr. aussi IV.51]). Le 3 du mois suivant, le IIème mois, Samsi-Addu écrit à Yasmah-Addu une lettre (I.53+M.7340) qui nous apprend que celui-ci continue sa campagne dans la région de Zalmaqum. De cette lettre, on apprend aussi qu'une armée de Samsi-Addu, revenue de Babylonie, s'est jointe aux 3000 Ešnunnéens et qu'ensemble ils vont régler l'affaire des Turukkum (cfr. I.5; IV.25 et surtout V.17+A.1882), puis Samsi-Addu prendra la tête des troupes et ils iront à Zalmaqum. Ainsi que nous l'avons vu, seul le nom de Turukkum apparaît dans les "Chroniques assyriennes". Peut-être faut-il ajouter ici deux lettres datées du 7.[II] et du 10.II (I.107; IV.35) qui montrent que Samsi-Addu, Išme-Dagān et Yasmah-Addu vont se réunir à Nahur. Ici, on est tenté de situer la victoire de la triade sur Larīm-Numahā, roi de la ville d'Aparḩā, qui est à situer dans la région de Zalmaqum (V.21; X.178). Or, la lettre V.21 émane de Sümu-epuh qui semble être en très bons termes avec Yasmah-Addu. On pourrait supposer qu'entretemps les relations entre les deux royaumes se sont améliorées.

L'année Adad-bani (1779/1778): Dans les "Chroniques assyriennes", on ne trouve ni l'éponyme Adad-bani ni les éponymes suivants. D'après les textes économiques de Mari, la présence de Yasmaḩ-Addu à Mari est attestée pour le IIème mois (XXV.198 [le jour manque]), pour le VIème mois (no. 76 [le jour manque]) et pour le 22.IX (XXV.113). Le 26.IX Yasmah̆-Addu va à Imār (VII.7). Trois visites sont attestées dans les textes économiques: le roi de Telmun le 21.IV (no. 61), le Grand Roi (Samsi-Addu) le 2.V (no. 97) et le 25.VIII les rois de Haššum et d'Ursum (no. 86). Les textes économiques de Chagar Bazar (cfr. aussi V.51) attestent des distributions de nourriture au cours du recensement

${ }^{185}$ XXVI/1, p. 248 , n. 85, pp. 255, 324c (XXVI.156) et sans doute aussi V.59 (cfr. XIV.104+. 5) $>$ V. $33>$ AREP, p. 122 b > IV. $88>$ IV. $74>$ II. $17>$ I. 123 . 
effectué par l'administration de Mari du 30.VI jusqu'au 6.VII. Ces archives attestent aussi la présence de Yasmaḩ-Addu à Chagar Bazar pendant la première moitié du IX ${ }^{\mathrm{ème}}$ mois. En supposant que Yasmah-Addu était présent à Mari lors des visites de Samsi-Addu et des rois étrangers dans la ville, on peut conclure que Yasmahn-Addu s'est trouvé à Mari du début de l'année jusqu'à la fin du VIIİ̀me mois, puis qu'il est parti pour un recensement dans l'Idamaraş, et qu'il est resté à Chagar Bazar jusqu'à la moitié du IXème mois. De là, il est retourné à Mari pour partir de nouveau en voyage, cette fois pour Imār, sur la boucle de l'Euphrate. C'est peut-être ici qu'il faut intercaler la lettre I.91+M.5461 ("Et comme tu te trouves à Tuttul et que tu es là tout près", 11. 6-7), qui atteste la disparition de Sūmu-epuh, ${ }^{186}$ suivie sans doute de l'accession au trône de son fils Yarīm-Addu. Le seul événement mentionné dans les lettres de Mari qui pourrait, peut-être, être daté, est la guerre commune d'Išar-Lim, à la tête de l'armée de Yasmaḩ-Addu, d'une force d'Ešnunna et d'une force babylonienne contre Malgûm (entre l'embouchure de la Diyāla et Kūt al-Imara). Deux lettres se rattachent à ces événements: I.27 et I.129, or dans I.129 on parle du recensement. Si c'est le même recensement qui apparaît dans le document de Chagar Bazar, ${ }^{187}$ on obtient une date de la victoire sur Malgûm. Le dernier éponyme dont le nom est conservé dans les "Chroniques assyriennes" est [Awīli]a. On pourrait postuler qu'il était suivi seulement d'un autre éponyme, donc Adad-bani. Durant cette année éponymale on devrait par conséquent placer la victoire remportée, probablement, à la porte de Saggarātum, par Yasmaḩ-Addu sur les Bini-Yamina, après la défaite de Yasmah̆Addu devant ces derniers à Șuprum, II.44; V.2.

L'année Nīmer-Sîn (1778/1777): D'après les textes économiques de Mari, le 6.VI on trouve Išme-Dagān en visite à Mari (no. 57) et le 6.XII un messager du Grand Roi (Samsi-Addu) se trouve à Mari (no. 59). Un document du 15.IV donne une liste des gens qui ont été transportés à Subat-Samaš depuis

${ }^{186}$ D'après D. Charpin et J.-M.Durand, M.A.R.I. IV, p. 310, l'événement date de l'éponyme Ikūn-pīya.

187J.-M. Durand, M.A.R.I. III, p. 142; D. Charpin et J.-M. Durand, M.A.R.I. IV, pp. 311, 316. 
l'Aḩ-Purattim (XXIII.595). Peut-être ces gens ont-ils été envoyés à Subat-Samš pour prendre part aux travaux de fortification qui sont mentionnés dans un "nom d'année" de Yasmaḩ-Addu (VIII.43).

L'année Tāb-şilli-Aššur (1777-1776): D'après des textes économiques de Mari le 27.VII Yasmah̆-Addu est allé à Ekallātum (no. 98), et le 8.I et le 20.XII la fille d'Išme-Dagān se trouve à Mari (VII.22;18), et enfin le 28.XII des messagers apportent des tablettes envoyées par Išme-Dagān (VII.21). Peut-être faut-il ajouter ici un autre document (no. 46) du 28 ème jour (sans mois), qui enregistre les quantités d'huile données à des gens qui sont venus d'Ekallātum. On constate, donc, pendant cette année des contacts étroits entre Yasmah-Addu et son frère Išme-Dagān. Il faut aussi noter que du 21.IX jusqu'au 10.X, on trouve YasmahAddu et son entourage à Terqa où a lieu une distribution d'armes et d'habits. On distribue aussi des armes aux habitants du district de Saggarātum le 14.X (XXII.222) et enfin le 18.X on distribue d'autres armes mais sans préciser le lieu (XXII.231). ${ }^{188}$ Ces distributions peuvent signifier qu'un danger menaçait le domaine de Yasmah-Addu, mais il n'est pas précisé d'où venait le danger.

L'année warki Tāb-şilli-Aššur (1776/1775): D'après des textes économiques de Mari, le Grand Roi se trouve à Mari le 1.II (VII.62) et le 1.V (VII. 81). Le 4.III on lui envoie de l'huile (VII.85). Des émissaires d'Ekallātum viennent à Mari le 16.I (VII.42) et le 20.II ils viennent de chez Išme-Dagān (VII.75). Le 15.II et le 11.IV, on atteste de nouveau la présence de la fille d'Išme-Dagān à Mari (VII.71; 31). Deux documents concernent des fugitifs qui arrivent à Mari au IVème mois, l'un venant de Dīr (dans le district de Mari) le 17 (VII.33) et l'autre venant de Tizrah (aussi dans le district de Mari) le 21 (VII.35). Le dernier document de l'année éponymale "warki Ṭāb-șilli-Aššur" est du 11.V (M.6088) et le premier document de l'époque de Zimri-Lim date d'environ trois mois plus tard, du 4 du mois de Nissan ${ }^{189}$ de 1' "année où Zimri-Lim est monté sur le trône de la maison de son père" (XXII.313; TH 82. 116). Il nous semble que peu après sa

\footnotetext{
188 J.-M. Durand, M.A.R.I. V, p. 185.

${ }^{189}$ VIII ème mois de l'année éponymale, ${ }^{\mathrm{er}}$ mois du calendrier mariote.
} 
visite à Mari au début du Vème mois le grand roi Samsi-Addu, fondateur du grand royaume mésopotamien, a disparu de la scène historique et qu'il a été remplacé par Išme-Dagān. Ainsi dans la "Stèle de victoire de Zimri-Lim" Išme-Dagān porte le titre officiel de la dynastie de Samsi-Addu "roi d'Ekallātum" (11. 2'-3'). ${ }^{190}$ La défaite de Yasmah̆-Addu et d'Išme-Dagān devant Zimri-Lim est décrite dans la "Stèle de victoire de Zimri-Lim"191, dans les lettres A.489.2'-4' et X.140.20-21 et dans le texte juridique A.3696.20.192 Des Babyloniens se trouvaient à côté de Yasmah-Addu jusqu'au jour de sa défaite devant Zimri-Lim ("Stèle de victoire de Zimri-Lim", 11. 21'ss.). Il faut supposer que la conquête de Mari par Zimri-Lim a eu lieu à un moment donné entre la seconde moitié de Vème mois et la fin du VIIème mois: Zimri-Lim "a triomphé d'Išme-Dagān et a expulsé Yasmahn-Addu de Mari" (A.489.3'-4')193. Le palais de Mari a été pillé: "lorsque Yasmah-Addu a été expulsé de Mari, le palais a été pillé" (X.140.20-21). Mais le harem était passé intact aux mains de Zimri-Lim. ${ }^{194}$ Après la conquête de Mari, Zimri-Lim attaqua la capitale d'Išme-Dagān ("Stèle de victoire de Zimri-Lim", 11. 14'-15').

Le royaume de Samsi-Addu était un vaste royaume centralisé, basé sur trois centres: Subat-Enlil (Tell Leilan) le siège du "grand roi", Ekallātum (à une journée de marche au nord d'Aššur) le siège d'Išme-Dagān, et Mari le siège de Yasmah̆Addu. ${ }^{195}$ Cette structure se manifeste clairement par la titulature de Samsi-Addu: "Prince de Mari, roi d'Ekallātum, gouverneur de Subat-Enlil" (A.889.9-11). Yasmah̆-Addu et Išme-Dagān, portaient tous les deux le titre de "roi", mais ils

${ }^{190}$ D. Charpin et J.-M. Durand, M.A.R.I. V, pp. 320-321 et D. Charpin, Miscellanea Babylonica, p. 60.

${ }^{191}$ Cfr. nos restitutions dans Bi. Or. (1987/1-2), p. 86.

${ }^{192}$ Nous n'avons pas pris en considération l' "Epopée de Zimri-Lim", car nous pensons qu'elle décrit la victoire sur les Bini-Yamina, cfr. la remarque de D. Charpin et J.-M. Durand, loc. cit., p. 325 , n. 139 .

${ }^{193}$ Cfr. aussi A.3696.

194J.-M. Durand, M.A.R.I. IV, p. 389 et dans Le système palatial, p. 85.

195J.-M. Durand et D. Charpin appellent ce royaume "Royaume de Haute-Mésopotamie", cfr. M.A.R.I. IV, p. 299. 
étaient à vrai dire des princes, des vice-rois, qui gouvernaient sous l'égide de leur père "le grand roi" Samsi-Addu. Išme-Dagān servait comme main droite de son père en l'aidant à gérer les affaires du royaume, et ainsi avait-il un grade plus élevé que son frère Yasmah-Addu. Il était responsable en outre de la partie orientale du royaume, la région de la Diyāla. Yasmah̆-Addu était responsable de la partie occidentale du royaume, qui couvrait plus au moins le royaume de Mari au temps de Yahdun-Lim, ainsi que d'une région au sud, sud-est du Djebel Sindjar. SamsiAddu ne se fondait pas sur la capacité de Yasmah̆-Addu à gérer proprement les affaires d'Etat, et ainsi il postait à ses côtés plusieurs de ses loyaux ministres pour qu'il l'aide: "mes anciens serviteurs, sur qui mes yeux se trouvent, je les ai mis à ton service" (I.113+.43-45). En outre, Yasmaḩ-Addu consulte souvent son père: "Présentement, que mon seigneur s'entretienne avec son père", écrit un fonctionnaire à Yasmahn-Addu (XXVI.292.23-24). Le statut de Yasmah-Addu se reflète dans une phrase d'une lettre qui lui est envoyée: "j'ai envoyé à mon seigneur (Yasmah-Addu) le doublet de ma tablette que j'avais fait porter au roi (Samsi-Addu)" (M.8002.7'-10'). Comme à l'époque de Yahdun-Lim, à l'époque de Yasmah-Addu aussi, dans cette partie du royaume qu'on peut appeler "le royaume de Mari", se trouvaient deux centres: Mari et Tuttul du Balīh. Dans trois lettres Yasmah-Addu rappelle sa nomination à Mari:196 "Pourtant, c'est Addâ (Samsi-Addu) qui m'a élevé; c'est à son ordre que mon destin a été fixé" (I.108.13-14), ou encore: "Addâ m'a installé à Mari" (I.113+.37-38). Une autre lettre fait mention de l'octroi de Tuttul à Yasmah-Addu: "je t'ai donné cette ville" (IV.11.10). Yasmah-Addu voulait étendre aussi son autorité sur Subat-Šamaš, près de Harrān. Išme-Addu a écrit à Yasmah-Addu à ce propos: "Au sujet de la demande au roi (de la ville) de Subat-Samaš que tu m'as écrit" (IV.27.5-6). Mais à la fin Yasmah-Addu n'a pas reçu la ville, et elle est resté sous l'autorité de Samsi-Addu. En dépit du fait que la ville de Tuttul était gérée directement par Yasmah-Addu, elle était néanmoins considérée comme une ville dépendante de Samsi-Addu: "Le palais de Subat-Samaš, en vérité, appartient-il à un autre roi ? Ou encore le palais de Tuttul appartient-il à un autre roi ? Ces palais appartiennent tous les deux à Addâ" (I.118.21'-23'). Le coeur du royaume de Yasmah-Addu,

${ }^{196} \mathrm{I} .3 .18 ’-20 ’ ; 108.4-26 ; 113+$. 
qui s'appelle "Ah-Purattim", s'est étendu de Mari jusqu'à Saggarātum, comme il ressort clairement de la lettre du devin Asqudum: "Dès mon arrivée même à Terqa, Tarim-Šakim en personne est arrivé, et je l'ai interrogé comme il suit: Zunan (un devin) a-t-il pris les présages pour le salut du pays et des forteresses ? ... je suis retourné avec lui à Saggarātum, et j'ai pris les présages pour le salut de la ville de Saggarātum ... Et aussitôt, à la forteresse de mon seigneur (Dūr-Yasmah̆Addu), à Terqa, à Șuprum et à Mari, je (les) prendrai" (XXVI.88.5-24).197

Le royaume était divisé en districts, dirigés chacun par des gouverneurs appelés šāpiţum. ${ }^{198}$ Les districts avaient leurs propres capitales avec les palais des gouverneurs. La nomination des gouverneurs a dû obtenir le consentement de SamsiAddu. En effet, Yasmah-Addu proposait le candidat, mais il devait recevoir l'approbation de son père Samsi-Addu. Les gouverneurs étaient choisis soit parmi les gens du district qu'ils devraient gouverner, soit parmi les hauts fonctionnaires, et on pouvait même transférer un gouverneur d'un district à un autre. Le gouverneur doit être d'après Samsi-Addu, "un homme de confiance que tu (YasmahAddu) choisiras, le meilleur du pays, qui est avisé et en qui tu as confiance" (I.9.16-18). A côté du gouverneur, on place "un chef de maison sûr, un fonctionnaire du cadastre sûr" (I.18.34-36). ${ }^{199}$ Les régions de la steppe, où paissaient les troupeaux, étaient soumises à l'autorité d'un fonctionnaire spécial, qui portait le titre de merhhum:200 "Il devra gouverner un vaste pays" écrit Samsi-Addu à Yasmah-Addu (I.62.10'). Le rôle des gouverneurs était avant tout de garder le district: "Garde la ville et le pays pour leur maitre" (I.18.30). En temps de guerre, les gouverneurs rassemblaient les gens de leur district dans les forteresses, et quand le roi s'apprêtait à partir en expédition, ils lui fournissaient des gens et des provisions. De temps en temps, ils devaient procéder au recensement de leurs gens, et ils pouvaient même juger les gens de leur district. Dans chaque district se

${ }^{197}$ Voir aussi A.2231.Rev.5'-6'; XXVI.158 et D. Charpin et J.-M. Durand, $R A$ LXXX, p.149. ${ }^{198}$ M. Anbar, Samši-Addu, pp. 102-103, 107-108; M. Marzal, JNES XXX (1971), pp. 186217; J.D. Safren, HUCA L (1979), pp. 1-15; J.-R. Kupper, RLA VII/5-6, p. 388.

${ }^{199}$ Et au temps de Zimri-Lim voir XXVI.398.18.

${ }^{200}$ M. Anbar, Samši-Addu, pp. 104-106; M. Marzal, ibidem, pp. 194-203; J.D. Safren, Or. LI (1982), pp. 1-29; D. Charpin et J.-M. Durand, RA LXXX, p. 152. 
trouvaient des champs et des troupeaux royaux, qui étaient soumis à la responsabilité des gouverneurs: ils veillaient à la culture de ces champs et à la moisson du blé, ils s'occupaient de la tonte des troupeaux, du traitement de la laine et du tissage des vêtements. Ils étaient aussi chargés de prélever les taxes sur les fermiers royaux, en plus des taxes qu'ils devaient prélever sur leur district pour le palais (XXI.200 [biltum]; XXVI.398.20 [šibšum]). ${ }^{201}$ Dans une lettre de SamsiAddu qui concerne ces taxes nous lisons: "En une année, un talent $(30 \mathrm{~kg})$, deux talents d'argent qu'il recueille. Ce n'est pas au moyen du blé, de l'huile et du vin qu'il vendrait régulièrement et qu'il rassemblerait cet argent ?" (I.73.12-17). A la disposition des gouverneurs se tenait le garde frontière, le sakbum, et probablement aussi des unités de l'armée royale. En cas de besoin, le roi fournissait aux gouverneurs des gens pour des activités civiles et militaires. Dans l'administration du royaume, le vizir était responsable aussi des gouverneurs, en créant un lien entre eux et le roi. Toute la hiérarchie administrative apparaît dans une lettre de Samsi-Addu à Yasmaḩ-Addu: "La tablette que Yašūb-El (le gouverneur) a envoyée à Lā'ûm (le vizir) et que Lā'ûm t'a envoyée, tu me l'as envoyée; je l'ai entendue (lire)" (I.47.5-8).

\section{Zimri-Lim (1775/1774-1761/1760)}

Zimri-Lim n'était pas d'origine royale. Il était le fils de Hatni-Addu et Addudūri. ${ }^{202}$ C'est seulement après son accession au trône de Mari qu'il s'est rattaché à la famille royale en s'intitulant "fils de Yahdun-Lim", et pour resserrer encore ses liens avec cette dynastie il donna à ses fils les noms de Yahdun-Lim et Yaggid-Lim. Mais pour Hammurabi, le roi de la Babylonie, il appartenait à la dynastie mariote. Ainsi dit-il au messager de Zimri-Lim: "auparavant son père et son aieul ne faisaient pas de rapports complets à cette maison" (XXVI.449.18-19). D.

${ }^{201}$ Voir aussi D. Charpin, M.A.R.I. II (1983), p. 90 qui fait référence à un document inédit "qui mentionne des quantités d'argent, des moutons et des boeufs (correspondant), aux charges de gouverneur, chef de cadastre et intendant", pour le šibšum cfr. aussi XXVI.265.20.

${ }^{202}$ D. Charpin et J.-M. Durand, M.A.R.I. IV, pp. 336-338. (*) 
Charpin et J.-M. Durand voient en Zimri-Lim un Bini-Sim'ālite. ${ }^{203}$ Leur avis est basé, lui aussi, essentiellement sur les paroles de Hammurabi qui se réfère dans une lettre à Zi-im-ri-Li-im LUGAL DUMU Si-im-a-al, qu'ils traduisent par: "le roi Zimri-Lim, un fils de Sim'al/Zimri-Lim, le roi Sim'alite" (RA LXXX, p. 150/ XXVI.385.6'). Or, il nous semble qu'on devrait traduire plutôt: "Zimri-Lim le roi des Bini-Sim'āl". 204 On peut comparer ce titre aux titres trouvés dans la fameuse lettre de Bahdi-Lim: LUGAL HAA-NA-MES LUGAL $\boldsymbol{A k}$-ka-di-im (VI. 76.2021). Zimri-Lim était le roi des Bini-Sim'āl, tout comme il était le roi des Huanéens et des Accadiens. A l'instar de ses prédécesseurs, il a aussi adjoint à son harem le harem de son prédécesseur, en l'occurrence, Yasmahn-Addu. ${ }^{205}$

Zimri-Lim, le nouveau roi de Mari, trouvait un changement considérable dans les relations internationales, en comparaison avec la situation du temps de SamsiAddu. Au temps de ce dernier, la Mésopotamie (la Djéziréh) était unie sous un régime centralisé fort et stable, qui avait conclu des alliances avec les pays voisins, dans lesquels Samsi-Addu représentait la partie dominante, le seigneur de ses rois-vassaux. A l'époque de Zimri-Lim le tableau a changé sans qu'on puisse le connaître: Mari n'était qu'une des forces qui luttaient pour l'hégémonie en Mésopotamie, et même pas la plus forte d'entre elles. La stabilité internationale s'ébranlait, ainsi que la stabilité à l'intérieur du royaume de Zimri-Lim. Plusieurs lettres de l'époque illustrent d'une façon remarquable la situation internationale et celle de Zimri-Lim. Itūr-Asdu, le chef de la garnison mariote à Nahur (à l'ouest du haut Habur) écrit, vers le milieu du règne de Zimri-Lim, une lettre, devenue classique, où il décrit le parallélogramme des forces lors de son discours aux rois de l'Idamaraş qu'il veut convaincre de rejoindre Zimri-Lim: "Il n'y a pas un roi, qui à lui seul, soit (réellement) puissant ! 10 (ou) 15 rois suivent Hammurabi, le roi de Babylone, autant Rīm-Sîn, le roi de Larsa, autant Ibal-pī-El, le roi d'Ešnunna, autant Amud-pi-El, le roi de Qatanum; 20 rois suivent Yarìm-Lim, le roi de Yamhad" (A.482.24-29). ${ }^{206}$ Zimri-Lim, quant à lui, décrit dans une lettre qu'il

${ }^{203}$ RA LXXX (1986), p. 150.

${ }^{204}$ Pour DUMU au lieu du pluriel DUMU-MES qu'on attendrait cfr. XVI/1, p. 30. (*)

205 J.-M. Durand, Le système palatial, p. 85.

${ }^{206}$ Cfr. aussi XXVI.303.20'-22' et XXVI/2, p. 58, n. j. 
adresse à son beau-père Yarīm-Lim, les perpétuelles agitations qui règnent dans son pays: "C'est mon père (Yarīm-Lim) qui m'a fait remonter sur mon trône, et c'est lui seul qui m'a soutenu et qui a consolidé la fondation de mon trône. Maintenant, depuis les jours nombreux où je suis remonté sur le trône, je ne livre que combats et batailles, et jamais encore je n'ai pu faire rentrer dans mon pays une récolte dans la paix" (A.1153.24-30). La troublante situation atteignait parfois le palais même. Kibri-Dagān, le gouverneur de Terqa, prie Zimri-Lim: "pendant trois (ou) quatre jours, que mon seigneur ne sorte pas de son palais !" (III.18.2527). Et enfin sa femme Sibtum lui transmet le présage du devin Selibum: "ZimriLim, on va t'éprouver par un soulèvement. Prends garde à toi. Place à tes côtés tes fidèles serviteurs, que tu aimes. Mets-les en faction et qu'ils te gardent. Ne déambule pas livré à [toi]-même" (XXVI.213.8-19). Les rapports internationaux de Zimri-Lim sont illustrés admirablement par le va-et-vient des messagers, par exemple, Bahdi-Lim, le gouverneur de Mari, demande à Zimri-Lim: "Des messagers, de passage, (venant) de Babylone, Ešnunna, Ekallātum, Karanā, Qabrā et Arraphāā, lesquels sont envoyés à Yamḩad, Qatanum, Hașurā et C[arkémi]š, vont arriver; dois-je les laisser aller, dois-je les retenir ? En outre, si des messagers (venant) de Yamhad, Q[atanum, Haşu]rā et Carkémiš, [lesquels sont envoyés à Babylone, Ešnun]na, [Qabrā, Arraph]]ā, [Ekallātum et Karanā arrivent; dois-je les laisser aller], dois-je les retenir ?" (VI.23.19-32).

Comme pour l'époque de Yasmah-Addu, nous allons décrire par la suite seulement les déroulements historiques dont on connaît, ou dont on peut au moins restituer, le cadre chronologique.

L'année ZL 0, l'année de l'accession au trône (1776/1775): Entre le 11.X et la fin de cette année, Zimri-Lim est monté sur le trône de Mari après avoir expulsé Yasmaḩ-Addu de la ville, et Išme-Dagān d'Ekallātum. Au temps de sa conquête de Mari, Zimri-Lim profita d'un soutien important de la part de YarïmLim, le roi de Yamhad, qui s'exprime ainsi: "C'est moi qui ai fait remonter ZimriLim sur son trône" (A.1153.8-9). L'alliance entre Mari et Yamhad était décrite par l'image suivante: "Le jour où un incendie s'allumera au pays de Yamhad, viens l'é[teidre] ! Mais le jour où un incendie s'allumera à Mari, c'est moi qui 
viendrai l'éteindre !" (S.115, no. 72-39). (*) Et en effet, Yarīm-Lim était considéré comme le père de Zimri-Lim (XXVI.148.8, 15, 4'), c'est à dire son suzerain.

L'année ZL 1 Bīt abišu/ Annunītum (1775/1774): A la fin du IVème mois (XXII.258), milieu du Vème mois (XXIV.53), on trouve Zimri-Lim en Idamaraș (le triangle du Habur) sans doute après avoir conquis la ville de Kaḩat (Tell Barri). ${ }^{207}$ Pour consolider son autorité en Idamaraș Zimri-Lim a marié sa fille Simatum avec son principal allié dans la région, Hāaya-Sūmû, le roi d'Ilānşurā. 208

L'année Kah̆at (1774/1773): Zimri-Lim a rétabli l'ordre dans l'Aḥ-Purattim. Il a quitté Mari le 27.X pour le district supérieur, probablement Terqa, et y est retourné le $15 . X I{ }^{209}$ Ce même mois Simaḩlānē, le roi de Kurdā, visite Mari. ${ }^{210}$ La contre-dot (terhatum) pour Sibtum a été envoyée au Xème mois (XXV.616; M.13254; M.13180). ${ }^{211}$

L'année ZL 1' (1773/1772): Zimri-Lim, aidé par la Babylonie,212 a battu les Bini-Yamina, qui ont eu le soutien d'Ešnunna, ${ }^{213}$ dans le district de Saggarātum, et a détruit les remparts des villes bini-yaminites de Mišlān du district de Mari, et de Samānum, du district de Terqa. La date de ce conflit précède le 26.VIII, car on sait qu'à cette date la ville de Mišlān est entre les mains de Zimri-Lim (XXV. 424). Dans la seconde moitié du XIIème mois, Zimri-Lim part pour Subat-Enlil (XXIII.370;214 514).

207 T.82.

${ }^{208}$ J.-M. Durand, M.A.R.I. III, p. 162-172; B. Lafont, CRXXX'̀me $R A I$, pp. 118-119.

${ }^{209}$ Voir en dernier lieu J.-P. Materne, M.A.R.I. II (1983), p. 196; ajouter XXVI.61 et XXVI.5 (cfr. XXVI/1, p. 204, note).

210Voir la note précédente.

${ }^{211}$ XXVI/1, p. 102.

${ }^{212 X X V I / 1, ~ p . ~} 140$.

${ }^{213}$ XXVI/1, pp. 139.

${ }^{214}$ XXIII, p. 293. 
L'année ZL 2' (1772/1771): C'est l'année du mariage entre Zimri-Lim et Sibtum, la fille de Yarīm-Lim, le roi de Yamhad. A la fin du Vème mois, début du VIème mois, elle voyageait de Halab à Mari, accompagnée d'Asqudum, qui a apporté à Halab le cadeau de mariage (biblum) (XXVI.10; 14; 15). ${ }^{215}$ Le premier document qui atteste la présence de Sibtum à Mari date du 30.VIII (XXIII. 445). Zimri-Lim est monté encore une fois en Idamaraş et a conquis la ville d'Ašlakkā216 et de Nahur. ${ }^{217}$ Par la suite les rois de l'Idamaraş, qui se sont rassemblés à Nahur devant Haāya-Sūmû déclarent: "Il n'existe pas d'autre seigneur et père que Zimri-Lim et Haāya-Sūmû" (XXVI.347.9-12). Pour resserrer ce lien avec Hāya-Sūmû, Zimri-Lim donna une seconde fille, Kirûm, en mariage à celui-ci (cfr. XXVI.303.24'), mais elle n'est partie à Ilānșūra qu'au milieu de l'année suivante. $\left(^{*}\right)$ Un document du 10.VIII s'occupe de femmes faisant partie du butin de Samānum et Raqqum qui ont été transférées du palais de Mari aux rois de Zalmaqum et d'Idamaraș (XXII.66.1-11), à Hāya-Sūmû le roi d'Ilāṣūra (11. 1219), aux filles du roi (11. 20-30; XXI.412). Le 26.IX, d'autres femmes BiniYaminites sont envoyées au Yamḩad, chez Sarraya, le roi d'Elahuut, à Kurdā, à Hatnu-rabi le roi de Qațțarā et à Sarrum-kīma-kalima, le roi de Razamā (XXII. 27+). Au IXème mois, est attesté le premier envoi d'étain à Yarīm-Lim, le roi de Yamḩad, et Amud-pī-El, le roi de Qatanum (VII.87). Le 3.X, est enregistré l'argent pour la statue de Zimri-Lim qui sera envoyée à Halab (XXII.248). Des envois de cadeaux à la fin de l'année témoignent des relations internationales de Zimri-Lim: avec l'ouest - Yamḩad et Qatanum, avec le nord - les rois de Zalmaqum et d'Idamaraş, avec l'est - Qațtarā, Razamā, les Turukkéens et avec le sud - la Babylonie et l'Elam. Par contre, Ibal-pī-El, le roi d'Ešnunna, a conquis Rapiqum, ${ }^{218}$ et ainsi il a menacé le Suhum, le district méridional de Zimri-Lim.

\footnotetext{
${ }^{215} \mathrm{XXVI} / 1$, pp. 97-98.

216Nom d'année ZL 3'.

${ }^{217}$ XXVI.346.6, sur l'importance de la ville de Nahur cfr. XXVI/2, pp. 42-43.

${ }^{218}$ Nom de sa $9^{\text {ème }}$ année et peut-être aussi XXVI.503-504 (cfr. XXVI/2, p. 446, n. a au no. 504).
} 
L'année ZL 3' (1771/1770): En ce qui concerne les événements de cette année, $\left({ }^{*}\right)$ notre connaissance se base non seulement sur les documents de Mari mais aussi sur ceux de Tell al Rimah. Tout d'abord il faut souligner que ZimriLim est absent de Mari du 6.II jusqu'au 9.X. ${ }^{219}$ Les événements que nous allons décrire ont commencé durant notre année et se sont poursuivis jusqu'à la moitié de l'année suivante. Qarni-Lim, le roi d'Andarig, s'est associé à l'homme d'Ešnunna qui est monté au triangle du Habur, ainsi qu'on l'apprend dans une lettre de ZimriLim, écrite à Hुatnu-rabi, le roi de Qațtarā et Karanā: "Qarni-Lim a envoyé un rapport complet à l'homme d'Ešnunna en disant: parce que j'ai conclu un accord avec toi, tous les rois des environs se sont révoltés contre moi et Zimri-Lim s'est joint à eux" (OBTR 3.6-11). Ešnunna a conquis Razamā (XIV.106) et SubatEnlil. ${ }^{220}$ Nous savons que le 23.VIII les alliés demeuraient à Subat-Enlil, qui était peut-être sous le patronage de Qarni-Lim (A.2821). Cette lettre nous apprend, en outre, qu'après son séjour à Subat-Enlil, l'armée d'Ešnunna est retournée dans son pays (cfr. aussi OBTR 5). Zimri-Lim a chassé Ešnunna, peut-être à la porte même d'Andarig (XXVI.303.26'-29').221 La ville de Qațarā, quant à elle, est évacuée au Xème mois. $222 \mathrm{Il}$ semble qu'après le départ d'Ešnunna, Qarni-Lim ait été attaqué par une coalition de rois (OBTR 3). Qarni-Lim appelle son allié Ešnunna à l'aide, et pour sauver Qarni-Lim, qui était assiégé par H̦atnu-rabi et ses alliés, Ešnunna s'est préparé à attaquer Mari elle-même (OBTR 2.3-19). D'après une autre lettre des mêmes archives (OBTR 4), Zimri-Lim demande de l'aide contre cette menace. ${ }^{223} 15$ mille Ešnunnéens avec Yaggih-Addu vont attaquer l'Ah-Purattim (X.155.11-17).224 L'armée ešnunnéenne a été conduite par le commandant Šallurum (XXVI.480.14-15).225 Elle a réussi à s'emparer de Huarbû

\footnotetext{
${ }^{219} \mathrm{XXVI} / 1$, p. 144.

${ }^{220} \mathrm{XXVI} / 2$, p. 39.

${ }^{221} \mathrm{XXVI} / 1$, p. 145; XXVI/2, p. 37, cfr. aussi A.2238.

${ }^{222} \mathrm{XXVI} / 1$, p. 144.

${ }^{223} \mathrm{XXVI} / 1$, p. 145.

${ }^{224} \mathrm{XXVI} / 2$, p. 35 , n. 39.

${ }^{225}$ XXVI/1, pp. 140, 144-145; XXVI/2, pp. 403, 443.
} 
(1. 9), de Yabliya (11. 4-5) et de Ḩanat où un gouverneur a été nommé.226 Elle a commencé de se retirer au plus tard avant le XIIème mois. ${ }^{227} \mathrm{D}$ 'après le nom de la 10 ème année d'Ibal-pi-El II, celui-ci a vaincu Subartum - les rois d'Idamaraș - et Ḩanûm - l'armée de Zimri-Lim. 228

L'année ZL 4' (1770/1769): D'après le nom de l'année suivante, nous savons que Zimri-Lim a effectué un recensement général dans le pays. Le recensement a eu lieu aux Xème et XIİ̀me mois. ${ }^{229}$ Une lettre qui fait allusion à cet événement nous apprend aussi que Zimri-Lim se prépare à reconquérir l'Idamaraș: "J'ai appris le recensement dans l'Ah̆-Purattim. Que mon seigneur donne des ordres sévères. Que mon seigneur prenne en main les troupes et qu'au printemps mon seigneur agisse pour ramener à ses côtés le pays d'Idamaraş tout entier" (II.130. 33-40). C'est peut-être ici qu'on doit introduire les lignes de 1'"Epopée de ZimriLim": "Jusqu'à ce que le roi eut atteint son but, et que l'Idamaraș, il eut courbé à ses pieds" (11. 112-113). C'est Sammetar qui part pour l'Idamaraș, à la seconde moitié de l'année, pour apaiser les esprits et obtenir la réconciliation avec QarniLim. ${ }^{230} \mathrm{Au}$ IVème mois Narāmtum la fille de Zimri-Lim est donnée en mariage à Sarriya, le roi d'Eluhtum (XXIII.84; 423; XXII.232). A partir du Vème mois on commence à libérer les Bini-Yamina qui ont été capturés en $Z \mathbf{L L} 1$ '.231

D'après une lettre de Hali-hadun du 23.VIII (A.2821), on sait que Qarni-Lim et les rois des environs de Subat-Enlil ont placé sur le trône un fils de Turumnatki, le roi de Subat-Enlil, à la place de son père. Enfin, le VIIème mois de l'année, ZimriLim a conclu une alliance avec Ibal-pī-El, le roi d'Ešnunna (A.4356.10'-13') et celui-ci est reconnu par Zimri-Lim comme son suzerain (XXVI.37.10). ${ }^{232}$ Après la retraite d'Ešnunna l'année précédente, Hammurabi, le roi de Babylone, qui avait

${ }^{226} \mathrm{XXVI} / 1$, p. 146 et n. 61, p. 149.

${ }^{227} \mathrm{XXVI} / 1$, p. 146.

${ }^{228} \mathrm{Cfr}$. XXVI/1, p. 150.

229J. Sasson, M.A.R.I. IV (1985), pp. 443-444.

${ }^{230} \mathrm{XXVI} / 1$, p. 151 et A.505.

${ }^{231}$ XXIII, pp. 476-505.

${ }^{232} \mathrm{XXVI} / 1$, p. 150; XXVI/2, p. 404. 
aidé Zimri-Lim a repousser Ešnunna, s'installe à Rapiqum, qui lui a été autrefois octroyée par Samsi-Addu, lequel avait pris la ville aux Ešnunnéens (XXVI.449.61-62). Quant aux villes de Hit, de Harbû et de Yabliya, il s'avère qu'elles ont été autrefois données à Zimri-Lim par le "père" de Hammurabi (11. 4950) et de Zimri-Lim (XXVI.362.43-44; A.2730.12), le souverain d'Elam. Maintenant Hुammurabi revendique Hit tout en cédant Yabliya et Harbû à ZimriLim (XXVI.449.60). ${ }^{233}$ Enfin, il faut souligner que Zimri-Lim a effectué au Vème mois des travaux de creusage dans le lit du Habur (XXIII.42.6-7).

L'année ZL 5' (1769/1768): Au IIIème mois Zimri-Lim a mené des travaux de réfection des remparts de Dūr-Yahdun-Lim du district de Saggarātum (S. 108, no. 787). Le VIème mois on le trouve à Šehnä/Subat-Enlil. ${ }^{234}$ Il est possible qu'il soit parti en voyage déjà le Vème mois, et qu'il soit resté en Idamaraș jusqu'au milieu du VIIème mois. ${ }^{235}$

L'année ZL 6' (1768/1767): Au premier mois de l'année la fille de ZimriLim, Hazala, est donnée en mariage à Sibkuna-Addu, le roi de Šudā (en Idamaraș) (M.8214). A la fin du VIIème mois Zimri-Lim visite la ville de Hanat, dans le Suhum. ${ }^{236}$ Enfin des rois vassaux rendent visite à Zimri-Lim: Sarrãya, le roi de Razamā, accompagné de Masum-adal de la ville d'Alilānum, Hazib-Ulme de la ville d'Azuhinum et le général de la ville de Șubat-Ištar (II.78). Et au 17.X. HāayaSūmû, le roi d'Ilānșūra, vient à Mari suivi par Qarni-Lim, le roi d'Andarig, lequel arrive au Xème mois. ${ }^{237}$ Avant leur départ pour Mari les rois se sont rencontrés avec les rois de l'Idamaraș dans Urgiš, puis à Nahur pour calmer l'Idamaraș (XXVI.352). ${ }^{238}$

${ }^{233}$ XXVI/1, pp. 149-150; XXVI/2, pp. 404, 443, 452 et cfr. aussi XXVI.40.10-13..

${ }^{234}$ M.11594; XXVI.360.4.

${ }^{235}$ Cfr. IX.124 (cfr. XIII.36); XXV.114.

${ }^{236}$ XI.250; XXI.110; M.10497; M.10645; .

${ }^{237}$ XI.207, 265; XXIII.216; VII.150; XXVI.352 et voir M.L. Burke, $R A$ LIII (1959), pp. 139 146.

${ }^{238} \mathrm{XXVI} / 2$, pp. $118-119$. 
L'annee ZL 7' (1767/1766): Des rapports entre Mari et l'Elam, qui se manifestaient par des cadeaux et des achats d'étain, sont attestés pour le Ier, IIème, VIème, VIIİ̀me et IX X̀me mois de l'année. Ces rapports culminent avec l'envoi d'une armée à l'aide de l'Elam. ${ }^{239}$ La présence d'une armée de Zimri-Lim auprès du souverain d'Elam est mentionnée dans une lettre du gouverneur de Saggarātum Yaqqim-Addu (XIV.122.26-27). Au milieu du Vème mois "Zimri-Lim et une bonne partie de sa cour sont allés dans le nord-est de la Mésopotamie",240 en Idamaraş. En effet on le trouve dans les villes d'Ilānșurā, de Tâdum, Razamā et Hušlā (VII.104; 117). La dernière ville constituait le but de son voyage. ${ }^{241} \mathrm{Au}$ cours de son déplacement il entre en contact avec Kabiya, le roi de Kaḩat, HăyaSūmû, le roi d'Ilănșurā, Qarni-Lim, le roi d'Andarig, Ḩammurabi, le roi de Kurdā, Sarrāya, le roi de Razamā, Sadun-šarri, le roi d'Azuhinum, et avec la ville de Karanā. Il est revenu de son expédition à la fin du Vème mois. Enfin, au IXème mois Kabiya, le roi de Kahat, et Yumraş-El, le roi d'Apilum, ont rendu une visite à Mari (M.11359+).

L'année ZL 8' (1766/1765): Les rapports entre Mari et l'Elam, qui se manifestaient par des cadeaux et des achats d'étain, sont attestés aussi durant l'année ZL 8', et cela pour le Ier, IIİ̀me, IVème, VIème et VIIİ̀me mois de l'année. Au début du XIIème mois de l'année, Zimri-Lim part en expédition et ceci va durer cinq mois. ${ }^{242} \mathrm{Le}$ but de cette expédition figure dans une variante de l'un des noms de l'année suivante (ZL 9'): "L'année où Zimri-Lim est monté au secours du Yambad" (S.108, no. 1316). Zimri-Lim est accompagné d'une partie de la cour: l'une de ses femmes, Yataraya, d'importants dignitaires et des orfèvres, tandis qu'à Mari Zimri-Lim a laissé le gouverneur Bahdi-Lim, afin qu'il garde le palais. L'itinéraire qu'a suivi l'expédition passait par Terqa, Saggarātum, Zilhan, Qardahat, Yakaltum, Hakkulan, Yabuhum, Zalpah ša mahirêtim, Yakullum, Tuttul, Hạ-

${ }^{239} \mathrm{D}$ 'après le nom de l'année suivante et A.2730.12-13.

240J.-M. Durand, N.A.B.U. 1987/98.

${ }^{241} \mathrm{XXVI} / 2$, p. 315 , n. a.

${ }^{242}$ P. Villard, UF XVIII (1986), pp. 387-412; XXVI/2, pp. 238-239. 
lab, Muzunnum, Layašum, Hazazar pour aboutir à Ugarit sur la Méditerranée. Au cours du voyage, Zimri-Lim échangea des cadeaux diplomatiques avec plusieurs rois des régions traversées, comme H̦āya-Sūmû, le roi d'Ilānșurā, Yawi-Ilā, le roi de Talhāyum, Dādi-ḩadun, le roi de Rabbûm, Šnnam, le roi d'Ursum, Aplaḩanda, le roi de Karkamiš, Yarìm-Lim, le roi de Yamḩad, Amud-pī-El, le roi de Qatanum, Yantin-Hammû, le roi de Gublā et Ibni-Addu, le roi de Ḩașurā. A Ḩakkulan, Zimri-Lim a rencontré son beau-père, Yarīm-Lim, sa belle-mère Gašera, et son beau-frère Hammurabi, et ensemble ils ont fait route jusqu'à Ugarit.

Le premier document qui concerne l'expédition atteste le séjour de Zimri-Lim à Terqa le 2.XII, le 8.XII, on le trouve à Saggarātum et le 19.XII il est déjà à Zilhan, qui est à situer dans le district de Qattunān.

L'année ZL 9' (1765/1764): Le 1.I Zimri-Lim se trouve encore à Zilhan. L'étape suivante était la ville de Qardahat, sur le Haut-Habur, en Idamaraş. La présence de Zimri-Lim dans cette ville est attestée le 10.I et le13.I. Il nous semble que la raison d'être du passage de Zimri-Lim par l'Idamaraș était de recruter une armée qu'il conduirait à l'aide de Yarìm-Lim. En effet, dans une lettre d'ItūrAsdu, le gouverneur de Naḩur, nous lisons "Auparavant, lorsque mon seigneur était allé à Ugarit, et que les troupes d'élite (bihrum) de l'Idamaras étaient parties avec mon seigneur" (A.2966+.16-18). Ces troupes d'élite étaient très probablement des Ḩanéens, comme il ressort clairement d'une lettre de Bahdi-Lim où les Hanéens sont justement qualifiés comme étant des "troupes d'élite" (bihrum) (VI.28.7-9). Notre supposition selon laquelle il s'agit des Hanéens, pourrait expliquer le rachat pour 3 mines 1/3 d'argent de quelque 25 Hanéens aux BiniYamina le III.ZL 9', sans doute à Ugarit (M.11351; XXIII.540). P. Villard suggère "que cette libération accompagnait une promesse d'alliance". Et en effet, une lettre du roi des Uprapéens, Hammi-ištamar, nous révèle l'existence d'une alliance conclue à Ugarit sans doute dans le temple de Addu de Halab (A.2094. 910). Notre supposition quant au but de cette expédition pourrait trouver un autre appui dans une lettre du gouverneur Kibri-Dagān, qui est à dater de la fin du IIİ̀me mois où celui-ci écrit: "Que mon seigneur vienne ici sain et sauf ... et ils (les Anciens de Terqa) prient pour mon seigneur et les armées" (III.17.14-20). A Ugarit, l'expédition resta environ un mois, puis elle retourna à Mari via Dūr- 
Sūmu-epuh. Le premier document qui atteste la présence de Zimri-Lim à Mari date du 6.V (M.11167). Lors du séjour de Zimri-Lim à Ugarit, des événements dramatiques se déroulaient dans "son pays", l'Idamaraş, et en Babylonie. A l'époque de la moisson, ${ }^{243}$ le IIÌ̀me mois, les relations entre l'Elam et Hammurabi d'un côté (XXVI.370.15'-16'; 303.49') et Rīm-Sîn, le roi de Larsa, de l'autre, se sont dégradées, et ils ont craint une éventuelle attaque de l'Elam (XXVI.367; 303.49'). ${ }^{244}$ Et en effet, une guerre éclata entre l'Elam et la Babylonie, secondée par Mari245 et Išme-Dagān (XXVI.384.23'). ${ }^{246}$ La bataille décisive s'est livrée dans la ville de Hirītum, sur l'Irnina, au sud de Sippar, ${ }^{247}$ et l'Elam qui assiégeait la ville (XXVI.384.23') fut vaincu (II.30+; XXVI.327.6'-7'; 328.21-22) et se replia vers son pays via Ešnunna qu'il pilla (XXVI.376. 6-10; 377.5-8; A.3073+). ${ }^{248}$ On parvient à dater le dernier événement grâce au recoupement de deux textes économiques XXIII.542 et M.8806.249 Après avoir conquis Ešnunna, les Elamites retournent dans leur pays et les Ešnunnéens choisissent un nouveau roi à la place d'Ibal-pī-El, un certain centenier ${ }^{250}$ nommé Șilli-Sîn (XXVI.377.516). Il faut aussi signaler qu'à cette époque, Išme-Dagān, le roi d'Ekallātum, qui se trouve dans un état physique pitoyable, a trouvé asile chez Hammurabi (XXVI.370.46' ss.), probablement après avoir été livré par les rois de Subartum

\footnotetext{
${ }^{243}$ XXVI.365.28-30, 38; 370.23'-25'.

${ }^{244}$ XXVI/2, pp. $159-160$.
}

${ }^{245}$ De cette époque date sans doute l'alliance entre Zimri-Lim et H̦ammurabi contre Șiwapalarhuuhpak (M.6435+M.8987).

${ }^{246}$ XXVI/2, pp. $150,154$.

${ }^{247} \mathrm{XXVI} / 2$, p. 34 et $\mathrm{n} .36$.

${ }^{248} \mathrm{XXVI} / 2$, pp. 34-35, 38, 150.

249J.-M. Durand "Fragments rejoints pour une histoire élamite" dans Fragmenta historiae Elamicae, Mélanges offerts à M.-J. Stève, L. De Meyer, H. Gasche et F. Vallat (éd.), Paris 1986, pp. 118, 121 et cfr. XXVI.361.27-28; 384.21' et XXVI/2, pp. 34, 146, 152, 159. Cette proposition est confirmée par un synchronisme entre Ibal-pī-El et Mari, voir ci-dessus Chapitre I, § $3 \mathrm{~A}$.

${ }^{250}$ En nous basant sur le document XXIII.435, nous proposons de traduire LÚ-GAL-KU 5 par centenier, LÚ-NU-BANDA par cinquantenier et UGULA-10 par dizenier. 
au souverain d'Elam qui se trouvait alors à Ešnunna (XXVI.384.20'-22'). ${ }^{251} \mathrm{Il}$ faut supposer que parallèlement, le IIIème mois (XIV.104+.18'), une force élamite à la tête de laquelle se trouvait Kunnam, est montée vers la Haute-Mésopotamie par Ekallātum (XIV.124.7') ${ }^{252}$ comme on lit dans une lettre de Huāya-Sūmu, le roi d'Ilānșurā, informant Baḩdi-Lim, le gouverneur de Mari, qu' "une troupe importante d'Elam et d'Ešnunna [est mont]ée vers le pays de Zimri-Lim, vers l'Idamaraș, et il n'y a personne qui puisse sauver le pays d'Idamaraș" (VI.66.48). ${ }^{253}$ En effet, nous constatons par une lettre du gouverneur de Saggarātum, Yaqqim-Addu, envoyée le 27.III, que des troupes d'Elam et d'Ešnunna accompagnées par Atamrum, le roi d'Allaḩad (XXVI.392. 23-24), 254 assiègent Razamā, la ville de Sarrāya l'allié de Zimri-Lim (A.2730. 7-8), après avoir conquis Ašnakkum, Susā, Andarig (XXVI.303.11'-12'; 305.2'-3') ${ }^{255}$ et SubatEnlil (XIV.104+; XXVI.307; 323.3-5). ${ }^{256}$ La conquête de Subat-Enlil, considérée, à cette époque, comme la ville du souverain d'Elam (XIV.104+), montre, nous semble-t-il, que l'Elam a essayé de créer un nouveau royaume à la place du royaume de Samsi-Addu, dont la capitale était justement la ville de SubatEnlil. La nouvelle de l'expédition de Kunnam est parvenue à Zimri-Lim très probablement le 5.IV dans la ville de Hazazar sur le chemin du retour (XXV.134.4). ${ }^{257}\left(^{*}\right)$ Quelques jours plus tard, déjà à Halab, Zimri-Lim essaie de gagner les faveurs de Šplarpak, le roi d'Anšan, et de Kudušuluš, le roi de Sušum, en leur envoyant des vases précieux (XXIII.542.22-30). ${ }^{258}$ Mais ces cadeaux n'ont pas eu l'effet escompté sur les envahisseurs et Zimri-Lim, après un court séjour dans sa capitale, dut partir pour sauver "sa ville" (XIV.103.8)

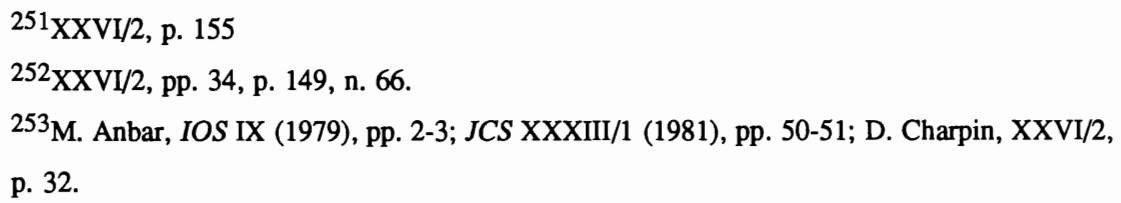


Razamā, probablement le 23.V (XXIII.545.11-14; XXVI.310.26-32; 311. 39). ${ }^{259}$ Hammurabi, le roi de Babylone, a envoyé à l'aide de Zimri-Lim "un contingent important" (VI.54), et en effet les documents administratifs attestent la présence de troupes babyloniennes à Mari le VIIIème mois. Il se peut qu'avec ce contingent, Hammurabi ait renvoyé à Zimri-Lim les troupes hanéennes qui lui avaient été autrefois envoyées par Zimri-Lim, accompagnées de troupes de Hammurabi, le roi de Yamḩad. Après son succès, l'Elam contrôla pour quelque temps l'Idamaraș (M.13014), et les rois qui étaient jadis des vassaux de Zimri-Lim se rendirent auprès de l'élamite Kunnam (XXVI.303). Mais l'aventure élamite s'est terminée assez vite, Elam et Ešnunna retournent chez eux, laissant à Ekallātum une garnison ešnunnéenne (A.655). ${ }^{260}$ Le seul vainqueur est Atamrum, le roi d'Allahad, qui annexe Andarig et Subat-Enlil (A.1925). ${ }^{261}$ Il faut souligner que pendant cette époque troublée plusieurs des vassaux de Zimri-Lim lui restent fidèles: HāayaSūmû, le roi d'Ilānșurā, Hammurabi, le roi de Kurdā et Ḩatnu-rabi, le roi de Qațtarā, Sarrum-kīma-kalima, le roi de Razamā-Yamutbal (A. 2730.5; A.3000.12$15),{ }^{262}$ et après la conquête d'Andarig aussi Atamrum. ${ }^{263}$

L'année ZL 10' (1764/1763): Selon D. Charpin, c'est vers le milieu de l'année qu'Išme-Dagān est retourné de la Babylonie à Ekallātum (A.649). ${ }^{264} \mathrm{Au}$ IIème mois on voit que le contingent babylonien, commandé par Nidnat-Sîn, qui avait été envoyé par Hammurabi, l'année précédente, était stationné à Mari (XIV.69; 70). ${ }^{265}$ Par contre un contingent bini-yaminite se préparait à partir le même mois pour la Babylonie (XXIII.428-429). D'après des textes administratifs et deux lettres de Yaqqim-Addu, le gouverneur de Saggarātum, nous savons que des troupes babyloniennes stationnaient dans le district de Saggarātum. Au IXème

\footnotetext{
${ }^{259} \mathrm{XXVI} / 2$, p. 36, n. 51.

${ }^{260} \mathrm{XXVI} / 2$, pp. $154-155$.

${ }^{261} \mathrm{XXVI} / 2$, pp. $43,242$.

${ }^{262} \mathrm{XXVI} / 2$, p. 41, n. 89.

${ }^{263} \mathrm{XXVI} / 2$, p. 248.

${ }^{264}$ Cfr. XXVI/2, p.41, n. 89, p. 155.

${ }^{265}$ XXI.100; 406; XXVI.470.15'; XXIII.564-567 cfr. XXIV.94 et voir XXVI/2, p. 375.
} 
mois a eu lieu la révolte de Kukkutānum, le commandant de l'armée d'AškurAddu, le roi de Karanā, qui fut réprimée avec l'aide du contingent Mariote (XXVI.412.2-58; 413.3-28; 511.14). ${ }^{266}$ C'est aussi l'époque d'une alliance entre Išme-Dagān et Hammurabi, le roi de Kurdā, d'un côté, et Aškur-Addu, Atamrum et Mari de l'autre. ${ }^{267}$ Durant cette année Hammurabi, le roi de Babylone, a infligé une défaite aux Elamites et à Ešnunna (nom de sa 30ème année) .

L'année ZL 11' (1763/1762): A la fin du Ier mois et au début du IIème mois Habdu-Malik a effectué une tournée dans les capitales de la région du sud de Djebel Sindjar: à Kurdā, à Andarig, à Karanā et à Andarig de nouveau avant de retourner à Mari avec les cadeaux qu'il a reçus des rois qu'il a visités: Hammurabi, Atamrum et Aškur-Addu (XXVI.392; M.11948).268 D'après le nom de l'année suivante, Zimri-Lim a conquis de nouveau la ville d'Ašlakkā et la ville d'Eluhtum de la haute Mésopotamie. En effet, au IIIème mois Zimri-Lim part vers le nord dans la région de Hurwaš, ${ }^{269}$ probablement pour faire face à la pénétration d'Išme-Dagān dans le pays allié de Zimri-Lim (XXVI.419). ${ }^{270}$ Le 29 Zimri-Lim se trouve à Zurrā, en Idamaraș. ${ }^{271}$ Le 8.IV un recensement de l'armée Hanéenne est effectué à Zurrã (M.5705). ${ }^{272}$ Au début du IVème mois on trouve Zimri-Lim dans la ville d'Urzikkā. 273 Dans la première moitié du Vème mois on trouve à Mari des hommes et des femmes, prisonniers de Șidqani et Hurwaš, qui sont

${ }^{266} \mathrm{XXVI} / 2$, pp. 236,245 , n. i, pp. $283,463,481$, n. d.

${ }^{267} \mathrm{XXVI} / 2$, p.157, n. 104 , p. 467.

${ }^{268} \mathrm{XXVI} / 2$, pp. $463,467$.

${ }^{269} \mathrm{XXVI} / 2$, pp. 209,240 et n. 19 , pp. 305,468 et n. 32 . Il faut signaler que d'après XXVI.404.43 on est avant la moisson, et dans XXVI. 519.17 on mentionne l'aire.

${ }^{270} \mathrm{XXVI} / 2$, p. 248 et n. 60.

${ }^{271}$ M.A.R.I. V, p. 617 cfr. aussi XXI.348 (le 22.IV à Našilā ) et XXI.3 (le 4.V à Ilănşurã et à Tãdum).

${ }^{272}$ Cfr. J.-M. Durand, M.A.R.I. V, pp. 616-617.

${ }^{273} \mathrm{XXVI} / 2$, p. 305. 
offerts aux divinités ou renvoyés à leur roi. ${ }^{274}$ Un document mentionne aussi "les ânes faisant partie des ânes représentant le butin de l'Idamaraṣ, du pays de Sudā" (A.3796.6-9). En Babylonie les relations entre Hammurabi et Rìm-Sîn, le roi de Larsa, se sont détériorées. Celui-ci a effectué des raids sur le territoire de Hammurabi (XXVI.372.27-32; 385.8'-9'). 275 A cette époque, Hammurabi considérait Zimri-Lim comme son principal allié (XXVI.385.5'-8'), et on constate qu'un contingent mariote, envoyé par Zimri-Lim, participait à cette guerre (XXVI.383.3-5; 386.6'-9'). Ipiq-Ištar, le roi de Malgûm prit lui aussi le parti de Hammurabi, tandis qu'il semble que Warassa, le roi de Dìr, a pris le parti de RīmSîn (XXVI.372.41-46; 373.29-32; 385.48'-49'). ${ }^{276}$ Durant le VIème mois Hammurabi a conquis d'abord Maškan-šapir277 après un bref siège (XXVI.383.6; 385.38'-44'), ${ }^{278}$ puis il assiégea Larsa (XXVI.383.11-12; 378.6$13 ; 381.15)$ et la conquit probablement au début du mois de Tichré (XXVI.386.10'; 381.20'-25'; 471.7-8, 8'-9'). ${ }^{279}$ Au moment du siège de Larsa, Išme-Dagān a demandé, sans succès, une aide militaire à Hammurabi contre une attaque de la part du roi des Turukkéens, Zaziya (384.25'-28'), 280 qui a pénétré jusqu'à la porte d'Ekallātum (XXVI.522.11'-12'; 425; 526.13-23), ${ }^{281}$ mais il y a une information non vérifiée encore d'après laquelle 4000 (ou: 6000) des hommes d'Ešnunna viennent de monter chez Išme-Dagān (XXVI. 378.17-21), ${ }^{282}$ sans doute à la place de 500 Ešnunnéens sous le commandement de Lipit-Sîn (ou:

${ }^{274}$ Cfr. P. Villard, M.A.R.I. V, 634; J.-M Durand, Le système palatial, p. 83, et n. 136 (M. 15275+,M.15241+,M.15125+); XXVI/1, p. 396 (A.3796) et cfr. aussi XXVI/1, p. 369, n. d.

${ }^{275} \mathrm{XXVI} / 2$, p. 147.

${ }^{276} \mathrm{XXVI} / 2$, pp. 153-154.

${ }^{277}$ L'actuel Tell Abu Duwari sur la rive droite du Tigre au nord de Nippur, voir P. Steinkeller, Abstracts de la XXXVI ${ }^{\mathrm{e}}$ RAI; E.C. Stone et P. Zimansky, Măr Sipri II/1 (1989), pp. 1-2.

${ }^{278} \mathrm{XXVI} / 2$, p. 148.

${ }^{279}$ Cfr. M. Anbar, $R A$ LXXII (1978), p. 117, 1. 22; Bi Or XXXV/3-4 (1978), p. 217, n. 1; N.A.B.U. 1989/83.

${ }^{280} \mathrm{XXVL} / 2$, pp. $155-156 ; 470-471$.

${ }^{281} \mathrm{XXVI} / 2$, pp. 314,505, n. f.

${ }^{282} \mathrm{XXVI} / 2$, pp. $155-156$, n. 98. 
Lipissa) qui a participé du IIIème mois au VIIème mois avec Išme-Dagān à l'expédition au sud du Djebel Sindjar dans le territoire d'Aškur-Addu, le roi de Karanā, ${ }^{283}$ mais il n'a pas reçu cette armée de renfort (XXVI. 428.7-12; 341). ${ }^{284}$ Zimri-Lim lui aussi demande une aide militaire de la part de Hammurabi. ${ }^{285}$ C'est aussi l'époque au cours de laquelle une alliance entre Ešnunna et Babylone fut négociée (XXVI.372.4-26), ${ }^{286}$ et Hammurabi a proposé une alliance à Atamrum, le roi d'Andarig (XXVI.372.47-80). ${ }^{287}$ Himdiya, qui gérait la ville d'Andarig lors de l'absence pour plusieurs mois d'Atamrum, est parti en expédition contre Hammurabi, le roi de Kurdā, et on le trouve à Subat-Enlil et à Amaz (X.84; XXVI.431-435). ${ }^{288}$ Au Xème mois Atamrum est revenu de la Babylonie avec 6000 Babyloniens. ${ }^{289}$

L'année ZL 12' (1762/1761): Au début de l'année on constate que Himdiya figure comme roi d'Andarig (S.143, no. 16 [= M.18156?]) et qu'un fort contingent babylonien sous le commandement de Mutu-Hatkim, monte avec Hulālum, le frère d'Atamrum (A.2603), et l'installe comme roi d'Allahad (II.122; XXVI.440-440-bis; 451). ${ }^{290}$ Cette expédition cause de l'inquiétude dans Suhum et dans Mari même (XXVI.209; 210; 212). ${ }^{291}$. Avant la montée des Babyloniens Zimri-Lim monte en Idamaraș (XXVI.185-bis.22) ${ }^{292}$ où il se trouve durant le IIIème mois (XXI.141; XXV.119); et le IVème mois (XXI.5; XXI.64; XXI.142);

${ }^{283}$ XXVI.426.6-7; 522.8-9; 523; 521; 520; A.1925; XXVI.423; 513; 427; 419; 525; 526.24 25, 34-35; II.40; XXVI.491.12 et XXVI/2, pp. 109-110, 209, 237, 240, 249, 312-313, 407, $465,467,469,471$.

${ }^{284}$ XXVI/2, pp.313, 409, 471.

${ }^{285} \mathrm{XXVI} / 2$, pp. 148,195 , n. e.

${ }^{286} \mathrm{XXVI} / 2$, p. 153.

${ }^{287} \mathrm{XXVI} / 2$, pp. $156-157$.

${ }^{288} \mathrm{XXVI} / 2$, pp. 327-328.

${ }^{289}$ XXVI.341.9; 438.6'; 496; 498.19-21; 499.5-6; 500 et XXVI/2, p. 409.

${ }^{290} \mathrm{XXVI} / 2$, pp. $212,242,246,350-351,360,410$.

${ }^{291} \mathrm{XXV} / / 1$, p. 401.

${ }^{292} \mathrm{XXVI} / 1$, p. 369, n. h et p. $441, \mathrm{n}$. 
il est retourné à Mari dans la première moitié du Vème mois ${ }^{293}$. Au VIIème mois Atrušipti, le roi d'Ursum et Hammu-labā, le roi d'Ašnakkum se trouvaient en visite à Mari (XXV.120 = N.A.B.U. 1988/2). Enfin, on constate que les relations avec Ešnunna se sont améliorées, car le 5.VIII un vase est envoyé à Șilli-Sîn, le roi d'Ešnunna (XXV.19). Par contre, durant cette année, Hammurabi a infligé une défaite à Ešnunna (nom de sa 32 ème année).

L'année de la prise de Mari par Hammurabi (1761/1760): D'après le nom de la 33ème année de Hammurabi, nous savons que durant cette année il a conquis Mari. Des documents babyloniens permettent de préciser que la conquête a eu lieu dans la première moitié de l'année, peut-être aux premiers jours de l'année.

A l'époque de Zimri-Lim, en dépit du fait qu'on l'a appelé une fois šar kiššatim "Le roi de la totalité" (XXVI.409.12) comme autrefois on a appelé Samsi-Addu, ${ }^{294}$ le royaume s'est beaucoup réduit, de sorte qu'il est devenu moins grand que le royaume du temps de Yahdun-Lim. Dans une lettre du haut fonctionnaire Sammetar, on trouve la description des limites du royaume: "[J'ai envoyé] immédiatement du secours jusqu'à Qattunān, jusqu'à Tuttul et en aval jusqu'à Suhum" (A.3006.26-29). Suhum est le district méridional dont la capitale était la ville de Hanat. Ce district s'est étendu jusqu'aux environs de la ville d'Ida (Hît), qui était soumise à l'autorité de Hammurabi, le roi de la Babylonie. La ville de Yabliya était une ville frontière du royaume de Zimri-Lim (XXVI.505.8-11; 506.5-7).

En amont de l'Euphrate, la ville de Tuttul marquait la frontière du royaume. La ville d'Abattum, qui était sise à une journée de marche au nord de Tuttul, s'est retrouvée entre les mains des Bini-Yamina (XIV.83.36-37). La ville d'Imār qui se trouvait dans la boucle de l'Euphrate, était entre les mains du roi de Yamhad (A.1153). On peut même supposer que le Balīh constituait la frontière entre le royaume de Zimri-Lim et les territoires des Bini-Yamina (A.1086.4-6). La ville de Dìr sur le Balịh était considérée comme ville-frontière (A.2057.35). Sur le Habur,

\footnotetext{
${ }^{293}$ XXI.143; XXV.120 = N.A.B.U. 1988/2; XXI.144; XXI.145.

${ }^{294} X X V I / 2$, p. 279 , n. d.
} 
la zone frontière du royaume de Zimri-Lim passait près de la ville de Qattunān (peut-être Tell Fadgami295) (XXVI.141.18).296

De même qu'à l'époque de Yasmahn-Addu, à l'époque de Zimri-Lim aussi le royaume était divisé en districts, et dans leurs palais qui se trouvaient dans les capitales fortifiées étaient installés des gouverneurs. Dans un document économique, on trouve l'énumération des palais des districts: "Le palais de Mari, le palais de Terqa, le palais de Saggarātum, le palais de Dür-Yaḩdun-Lim, le palais de Qattunān" (VII.277.V', la suite de l'énumération manque). On constate que cette liste est presque identique à la liste des villes fortifiées du temps de Yasmah-Addu (V.65.17-24): Saggarātum, Dūr-Yasmah̆-Addu (= Dūr-Yahudun-Lim), Terqa, Șuprum et Mari. Dans la liste de l'époque de Yasmah-Addu manque Qattunān, et dans celle de Zimri-Lim manque Șuprum, qui était une ville fortifiée mais non pas une capitale de district. La comparaison nous montre qu'aucun changement radical dans la division des districts n'avait eu lieu au temps de Zimri-Lim, et que bien au contraire celui-ci avait maintenu la division existant à l'époque de Yasmah-Addu. Trois parmi ces districts, qui constituaient le coeur même du royaume de ZimriLim - les districts de Mari, de Terqa et de Saggarătum - se trouvent dans une liste de fournisseurs de vêtements au palais de Mari (XXII.127.8-12), d'après une autre liste ils ont reçu des bois du palais de Mari (XXII.303). Enfin, les juges de ces trois districts apparaissent dans un document économique (XXV.783, la suite de la liste manque).

Au territoire de Zimri-Lim, il faut ajouter une région à l'ouest du Habur supérieur, qui était encore comprise dans le royaume de Mari à l'époque de Yahdun-Lim. A ce moment, son gouverneur résidait à Talhāyum, et les villes de Talhāyum et d'Ilānşūra étaient considérées comme "villes-frontières de Zimri-Lim" (XIII. 147.27-28; XXVI.301.5-6).

L'influence politique de Zimri-Lim s'étendait jusqu'aux pays d'Idamaraş (le triangle du Habur)(*) et de Zalmaqum (la contrée montagneuse au nord de la ligne Nisibin - Harrān), qui parfois étaient considérés comme les pays de Zimri-Lim. A l'époque de Samsi-Addu, l'Idamaraş constituait le coeur même de son royaume,

${ }^{295}$ H. Kühne, AfO XXV (1974/1977), p. 255.

${ }^{296} \mathrm{Cfr}$. XXII.313 du 4.I.ZL 1: un devin de Zimri-Lim se trouve à Qattunăn. 
où se trouvait sa capitale Šubat-Enlil. L'époque de Zimri-Lim a vu un changement considérable dans la structure politique de ce pays. Dans les villes qui étaient autrefois les sièges des gouverneurs, on trouve maintenant des rois indépendants. ${ }^{297}$ Un cas intéressant concerne Atamrum le roi d'Allahad et d'Andarig. Cet Atamrum était le fils d'un gouverneur du temps de Samsi-Addu du nom de Warad-Sîn, dont un autre fils, Hulālum, est monté sur le trône d'Allaḩad après Atamrum. Au début de son règne, Zimri-Lim envoie à Tiš-Ulme, le roi de Mardamān, et aux autres rois de l'Idamaraş, une lettre dans laquelle nous croyons reconnaître leur nomination par le roi de Mari: "Maintenant écrivez-moi et je viendrai et je vous prêterai un serment solennel, et donnez-moi une ville pour que je la rende à son maître, et quant à vous, je vous établirai, vous et vos biens, là où vous me le direz" (S.115, no. 72-15.12-20).298 Cette lettre nous apprend que le pays d'Idamaraş tout entier est attentif aux ordres de Zimri-Lim, et dans une autre lettre on parle du "pays d'Idamaraș, (pays) allié" de Zimri-Lim (XIV.51.11-12).299 De même on voit les rois d'Idamaraş se rassembler devant Ḩāya-Sūmû, le roi d'Ilānșurā, dans la ville de Naḩur en déclarant: "Il n'existe pas d'autre seigneur et père que Zimri-Lim et Ḩāya-Sūmû. Nous ferons tout ce que dit notre seigneur Zimri-Lim" (XXVI.347.9-15), et Atamrum, le roi d'Andarig, considère ZimriLim comme son "frère aîné" (XXVI.409.48-50; 410.13'). Par une lettre de HāyaSūmû, le roi d'llānșurā, nous apprenons que Zimri-Lim va nommer Ibal-Addu, le roi d'Ašlakkā, et le roi d'Ašnakkum, sans doute Šadum-labā (II.62. 12'-13'). De même, nous apprenons de la bouche d'Ibni-Addu, le roi de Tādum que "ZimriLim m'a installé comme roi de Tādum" (XXVI.310.6-7). Il arrivait même que Zimri-Lim servît d'intermédiaire dans les cas de litige entre les rois: "Auparavant, Turumnatki (le roi d'Apum) avait péché et s'était [montre hos]tile envers ḨāyaSūmû (le roi d'Ilānșurā). Maintenant, par l'ordre de mon seigneur en personne, Ḩāya-Sūmû a accepté une alliance avec Turumnatki" (X.5.13-17). Vers la fin de son règne son influence dans le nord était toujours grande comme il en ressort des

${ }^{297}$ Cfr. D. Charpin, "Les Elamites à Subat-Enlil" dans Mélanges Stève, 130; D. Charpin et J.M. Durand, RA LXXXI (1987), p. 136 et aussi J.-M. Durand, $R A$ LXXXII (1988), p. 97 s.

${ }^{298}$ M. Birot, "Reflets des Deux Fleuves", Louvain 1989, pp. 21-25.

${ }^{299}$ Cfr. aussi XIV.79.23-24. 
paroles d'Atamrum: "Il n'y a pas d'autre roi que Zimri-Lim, notre père, notre frère aîné, et celui qui marche à notre tête" (XXVI.404.17-18), et il est même considéré comme "le roi du Haut-Pays" (11. 25-26). Le pays d'Andarig est considéré comme un "pays de Zimri-Lim" (XXVI.433.21), et la ville de Talhaãyum est la ville de Zimri-Lim (A.2417.6, 45-46). (*) Ses alliés lui ont envoyé une aide militaire: 13 rois d'Idamaraş lui ont envoyé 1218 de leurs gens (IX.298),300 et Zimri-Lim de son côté leur envoie des renforts: "La ville de Ilānşurā, le roi Ḥāya-Sūmû et les troupes que mon seigneur a envoyées pour la forteresse vont bien" (II.135.5-8). Ou encore: "[De même que] tu as fait entrer une troupe à Nahur, maintenant fais (en) rentrer à l'intérieur de Talhāyum" (A. 2417.20-23) écrivent les Anciens de Talhāyum à Zimri-Lim. Celui-ci traversait le pays en concluant des alliances avec ses rois et en nommant des résidents. ${ }^{301}$ Mais là aussi sa domination n'était pas stable, comme nous l'apprenons par une lettre de Yawi-Ilā, le roi de Talhāyum: "Puisque le pays de Zalmaqum est dans le trouble, je veux jeter le trouble chez eux afin de pouvoir ramener le pays aux mains de mon seigneur" (XIII.146.21-24). Les rois d'Idamaraș, de Zalmaqum ${ }^{302}$ et du sud du Djebel Sindjar étaient les alliés de Zimri-Lim, et plusieurs d'entre eux se sont mariés avec ses filles (entre parenthèses sont notées les dates des mariages): 303 Ibbatum avec Atamrum, le roi d'Andarig (ZL 9'), Inib-šarri avec Ibal-Addu, le roi d'Ašlakkā (ZL 3'), Hazala avec Sibkuna-Addu, le roi de Sudā (ZL 6’), Narāmtum avec Sarrāya, le roi d'Eluhuut (ZL 4'), Sìmatum et Kirûm avec Ḥāya-Sūmû, le roi d'Ilānșurā (avant ZL 1'; ZL 2'), Tizpatum avec Ili-Ištar, le roi de Sunā. Les alliés de Zimri-Lim étaient, soit ses égaux "frères", comme Habbum, Sibkuna-Addu, le roi de Sudā, et Amudpī-El, soit ses vassaux "fils", comme Kabiya, le roi de Kaḩat, Yumraş-El, le roi d'Apilum, Arriwuk, Hāya-abum, le roi d'Apum et Adal-šenni, le roi de Burundum, ou "serviteurs', comme Yawi-Ilā, le roi de Talhāyum. Zimri-Lim a envoyé ses chefs d'armée avec des garnisons auprès des rois d'Idamaraș: Haqba-

\footnotetext{
${ }^{300} \mathrm{XXVI} / 2$, p. 83 , n. d.

${ }^{301} \mathrm{X} .121 ; 140.4-15$; XIII.145.14-16, 24-27; 147.5-9; 148.8; B.308.9-14.

${ }^{302}$ Cfr. p. ex. la lettre A.2417 où est mentionné le fait que Zimri-Lim a prêté serment au Zalmaqum et à l'Idamaraş (11. 38-40).
}

${ }^{303}$ B. Lafont, CRXXXIII ${ }_{R A I}$, p. 120. 
ahum chez Ḩammurabi, le roi de Kurdā, Uštašni-El puis Yamșûm chez ḨāyaSūmû, le roi d'Ilānşurā, Yasīm-El, chez Atamrum, le roi d'Andarig, Iddiyatum chez Aškur-Addu, le roi de Karanā et Huaqba-ahum, chez Huammurabi, le roi de Kurdā. ${ }^{304}$ Les rois chez qui ces garnisons stationnaient étaient responsables de leur entretien. ${ }^{305}$ Zimri-Lim envoyait parfois ses troupeaux paître en Idamaraș (AREP., p. 109), à la lisière du Zalmaqum (II.35.7-8, 19) et au sud du Djebel Sindjar (A.638+).

Dans le royaume même, les gouverneurs des districts ont été nommés à leur poste par Zimri-Lim, comme il ressort d'une lettre de Yaqqim-Addu, le gouverneur de Saggarātum: "avant que mon seigneur (Zimri-Lim) ne m'eût désigné (pour administrer) le district de Saggarātum" (XIV.18.11-12). Les gouverneurs, comme les autres fonctionnaires de l'Etat, étaient considérés comme "les serviteurs de Zimri-Lim", et ils s'appelaient entre eux du nom de "frère" (III.65.1-4).

Il faut souligner que, de même que Zimri-Lim a continué de maintenir la division du pays en districts, comme elle existait au temps de Yasmah-Addu, il gardait aussi dans son administration des anciens fonctionnaires de l'ère de YasmahAddu.

${ }^{304}$ D. Charpin et J.-M. Durand, RA LXXXI (1987), p. 132, n. 22; XXVI/2, p. 130, n. 3. ${ }^{305}$ XXVI/2, p. 48. 


\section{Chapitre III: L'organisation tribale}

\section{Introduction}

Dans ce chapitre nous allons examiner d'une part ce que l'on sait au sujet de la structure des tribus amurrites de Mari, et d'autre part nous allons étudier les termes que les scribes ont employés pour désigner les diverses unités tribales. Cette information sera comparée avec ce que nous savons à propos des structures de la tribu bédouine ${ }^{306}$ et de la tribu biblique. ${ }^{307}$ Nous ouvrirons notre discussion par la description de ces structures.

La plus petite unité sociale et économique chez les bédouins est la famille patriarcale, le 'ā'ilā. La famille habite dans une maison ou une tente, elle est dirigée par le père, et elle est identifiée par le nom du père. Lors de la transhumance, la famille circule ensemble.

Plusieurs familles, ayant un lien de parenté entre elles, forment une unité tribale qui porte le nom ḩamūlā ou firqā "section, fraction", qui est une unité sociale et économique unie. Le groupe porte le nom de l'un des pères d'une génération proche, et à sa tête se trouve un chef, le ra'is de la hamülā. Dans les tribus semi-nomades, la hamūlā habite ensemble dans un quartier du village.

Plusieurs hamāūlā 's se joignent pour former la sous-tribu, le fahid, dont le nom apparaît sous la forme du pluriel. A la tête de la sous-tribu se tient un cheikh, qui est reconnu par les ra'is's. La sous-tribu agit en commun seulement quand se pose un problème spécial. Chez les tribus semi-nomades, une sous-tribu habite dans un village.

L'ensemble des sous-tribus forme une tribu, le 'ašīiā. La tribu porte le nom d'un éponyme, et les membres de la tribu sont liés entre eux par des liens généalogiques.

306 M.P. von Oppenheim, Die Beduinen, p. 23; H. Charles, Tribus moutonnières, pp. 154-155;

L. Stein, Die Schammar-Gerba, pp. 142-146; V. Müller, Bédouins, p. 198.

${ }^{307}$ Nom 1:2; Deut 29:17; Jos 7:14-24; Jug 9:1; 1Sam 10:20-21. 
Chaque tribu possède une marque particulière pour marquer les animaux, le wasm, et un cri de guerre typique.

L'ensemble des tribus forme une confédération tribale, le qabīlā. La confédération tribale doit avoir un intérêt commun, un cheikh supérieur, qui représente la confédération tribale dans ses relations avec l'extérieur, un nom commun, et un sentiment d'homogénéité, qui se forme par un arbre généalogique. La généalogie peut être vraie ou imaginaire. A la confédération tribale se joignent des éléments étrangers.

Dans la Bible on trouve la structure tribale de la façon la plus claire dans le récit de l'acte d'infidélité d'Akân, Jos 7:14 "Vous vous approcherez au matin, par tribus, et la tribu que le Seigneur aura marquée s'approchera par clans, et le clan que le Seigneur aura marqué s'approchera par maisons, et la maison que le Seigneur aura marquée s'approchera homme par homme". On voit donc la séquence suivante: la tribu šèbeth le clan mišpāhā, la maison bayit et enfin l'homme geber.

Après avoir passé en revue les structures tribales bédouines et bibliques, on peut examiner la question de la structure tribale des tribus amurrites de Mari pour voir si à Mari on trouve les mêmes notions. Le premier terme tribal à étudier est le mot amurrite gā'um ou gāyum. ${ }^{308}$ Le gāyum s'applique tout d'abord aux clans des tribus des Hanéens, par exemple ga-yu Ya-ka-li-it "clan Yakallitum" ou ga-yu A-mu-rum "clan Amurrum" (TEM III, pp. 16-19, tablette AB ${ }^{309}$, col.II.4, 44). Mais le même terme se réfère aussi aux tribus Bini-Yamina et Bini-Sim'āl ellesmêmes, comme on peut s'en rendre compte à la lecture d'un passage d'une lettre de Sîn-tiri: "les Hanéens qui ont traversé, Bini-Sim'āl (ou) Bini-Yamina, quelle est leur tribu (ga-a-šu-nu ma-an-nu-um) ?" (A.2560.6-8). Dans une lettre, citée partiellement par J.-M. Durand, on trouve la phrase suivante: "J'ai fait faire les présages pour les habitants de la ville (Hurrān), clan par clan (ga-yi-ša-am)" (A.3993.37'-38'). C'est la première fois qu'on trouve le trait typique de l'installation des nomades dans des villes. Les nomades s'installent dans les villes d'après leurs divers clans, sans doute dans des quartiers spéciaux (cfr. ci-dessous

${ }^{308} \mathrm{Cfr}$. XVI/1, p. 73; $C A D$ G, p. 59a; $A H w$, p. $284 \mathrm{a}, 1556 \mathrm{a}$; Ph. Talon, Miscellanea Babylonica, pp. 278-280. Pour les deux formes du mot cfr. le mot amurrite ayarum ou ha'arum qui correspond à l'hébreu 'ayir.

309Un document parallèle est M.13204. 
Chapitre IV, § 4). Un autre terme qui se réfère à une unité tribale est le terme hibrum. ${ }^{310}$ Itūr-Asdu, le préfet du palais de Mari, écrit à Zimri-Lim à propos des Sutûm: "un hibrum de Hुammi-telû [et] de Yapahum [1?] cent Sutûm sont arrivés ici (à Mari) de chez Simahlānē (le roi de Kurdā d'Idamaraș)" (A.2801.14-19). Hibrum apparaît encore dans cinq documents, dans trois cas dans des passages endommagés (I.119.10; VII.267.2'; IX.244.1[?]), qui ne permettent pas d'en faire usage. Dans le quatrième document (VIII.11) il s'agit d'un acte juridique qui règle l'octroi d'un champ à un membre de Bît-Awin, un sous-clan des Hanéens (?, voir ci-dessous $\S 1$ ). Une partie des membres de Bīt-Awin habite la localité d'Appān: 5 mārū A-wi-in wa-aš-bu-ut Ap-pa-an (ll. 9-10) et une autre partie sont des nomades: 8 mārū $A$-wi-in hi-ib-ru-um ša na-wi-im (11. 20-21). Nous avons affaire ici à un sous-clan qui comprend huit familles, dont cinq sont sédentaires et huit sont en transhumance dans la steppe. ${ }^{311}$ Le cinquième exemple concerne lui aussi les Hanéens. D. Charpin et J.-M. Durand citent une expression d'un fragment inédit (M.5172): Hanûm ḩibrum ša nawîm. ${ }^{312}$ (*) Est-ce que le hỉbrum désigne lui aussi une entité tribale (clan ou tribu) comme gāyum, ${ }^{313}$ ou au contraire, désigne-t-il un simple groupe humain comme le heber biblique et la $h^{a} b u \overline{r a ̄}$ michnaïque ? Nous pensons que la seconde possibilité est préférable et que le hibrum serait un groupe nomade peut-être en transhumance. ${ }^{314}$

Au terme de l'étude de la structure des tribus amurrites à Mari on arrive à la conclusion que la terminologie tribale est moins développée que celle des bédouins ou celle de la Bible. Le seul terme vraiment tribal est gāyum, car bĩt abim se réfêre aussi aux sédentaires. ${ }^{315}$

${ }^{310}$ Cfr. XV, p. 202; $C A D$ H, p. 181a; $A H w$, p. 344a, p. 1561a.

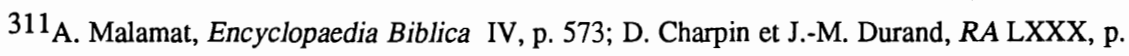
154 et n. 68 .

312 ibidem.

313J.-R. Kupper, Nomades, p. 20, n. 1; CAD H, p. 181a et $A H w$, p. 344a.

${ }^{314}$ Cfr. D. Charpin et J.-M. Durand, RA LXXX, p. 154 , n. 68 et p. 175 , n. 158.

${ }^{315}$ Cfr. CAD AI, pp. 73-75. 


\section{Hanûm}

Les Ḩanéens étaient divisés en huit ou neuf clans. Les noms de sept clans apparaissent dans une liste de distribution d'huile pour les garnisons de Mari et de Șuprum, qui se trouve dans le district de Mari (TEM III, pp. 16-19, tablette AB). Dans ce document, qui est divisé en huit colonnes, sont énumérés 222 "Hูanéens résidant à Mari" (V.4-8), et 36 "Ḥanéens résidant à Ṣuprum" (V.56-59), ces deux groupes comprenant 258 hommes "Ḩanéens résidant à Mari et à Ṣuprum" (V.6265). Les gens qui figurent dans la liste sont répartis en sous-groupes, des clans, des gāyum's. Les gens de Mari comprennent 30 hommes du clan Yer' $\mathbf{u}^{316}$ (I.32-35), 27 du clan Yakallit (II.3-5), 35 du clan Amurrum (II.42-45), 12 du clan Yabasu (II.59-60), 29 du clan Naḩanum (III.29-32), 7 du clan Niḩad (III.41-42), 24 d'un clan dont le nom manque (III.68-70), 15 du clan Ibalahu (IV.20-22), 43 d'un clan dont le nom manque (IV.[67-70]). Les résidents de Șuprum comprennent 9 hommes du clan Yabasu (V.19-20), 9 du clan Nihad (V.30-31), 9 d'un clan dont le nom manque (V.41-42) et 9 du clan Yer'u (V.52-53). Deux noms de clans résidant à Mari manquent tandis qu'un seul nom de clan résidant à Șuprum manque. Il se peut que les clans qui manquent à Mari figurent parmi les clans de Șuprum et vice versa, de sorte qu'à Mari et Șuprum résidaient les membres de sept clans. Dans

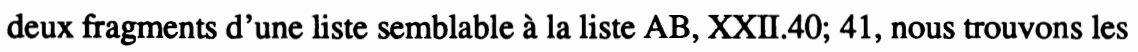
noms de quatre clans: Yakallit (40.II'.15'), Ibalahu (41.I'.7'), Nahan (41.II'.7') et Niḩad (41.II'.19'). Deux autres clans, Yabasu et Amurrum, qui figurent dans la liste $\mathrm{AB}$, apparaissent dans une liste de sugāgū, XXIV.235.5, 8. Jusqu'à maintenant nous avons compté sept clans. A ces sept il faut ajouter un huitième clan, Sibum. Dans certains documents représentant des listes de payements et de dettes des sugāgū (VII.227; IX.248; XXIV.58, 61, 62; XXV.760), les sugāgū en question appartiennent aux clans suivants: Amurrum, Ibalahum, Naḩanum, Niḩad, Sibum, Yabasu, Yakallit et Yer'u. Du fait que tous les clans sauf celui de Sibum

316 Lecture de Ph. Talon, op. cit., p. 282. J.-M. Durand (M.A.R.I. V, p. 232) lit Ya-bi-sa, qui serait une variante de Ya-ba-sa. Mais il faut noter que la variante apparaît par deux fois dans le document, et il est peu probable que le scribe emploie les deux formes dans un même document. 
soient identifiés comme nous l'avons vu comme appartenant aux Hanéens, nous pouvons déduire que lui aussi est un clan hanéen, le huitième.

Ces listes de sugāgū soulèvent le problème suivant: les sugāgū sont qualifiés soit par le nom d'un clan, soit par le nom d'une localité, soit encore par un nom propre tel que Mutatar homme de (LÚ) Abi-nakar (IX.248.Rev.11'; XXIV.61.III.4'), ou tel que Ani-Lim et Layasum hommes de Abi-nakar (XXIV.58.1-6), et encore Ibalpī-El homme de Nisiya[X] (XXIV.58.8-9). Ani-Lim et Layasum sont qualifiés dans un document comme appartenant à la tribu Hुanûm (XXIV.23.II'.9, 15) et dans un autre document encore comme appartenant au clan Yabasu (M. 11837). De ce fait Ph. Talon a tiré la conclusion que "Abi-nakar pourrait être un sous-clan du groupe de Yabasu."317 En comparant le cas d'Ibal-pi-El au cas d'Ani-Lim et Layasum, Ph. Talon propose de voir dans Nisiya[X] aussi un sous-clan Hanéen. ${ }^{318}$ Il nous semble préférable de voir dans ces noms des noms propres et non pas des noms de sous-clans: à l'exemple d'Ani-Lim et de Layasum, qui sont explicitement attribués au clan Yabasu, on peut ajouter l'exemple de Rip'i-Addu, qui dans un document (XXIV.54) est qualifié d'"homme (LÚ) d'Ibal-El" et dans trois autres cas il est qualifié de "fils (DUMU) d'Ibal-El le yer'éen" (VII.227. 16'; XXIV.62.I'.14'-15'; M.8575+.II). Ph. Talon cite encore dans son article le cas de Hammu-tar le sugāgu de I-sa-ri-i, qui figure dans trois documents. ${ }^{319} \mathrm{Or}$, dans l'un de ces documents, le nom I-sa-ri-i est suivi du déterminatif KI, ce que nous inclinerait à croire qu'il s'agit d'un terme géographique.

Il est possible qu'un document (XXIII.87.1-36) nous ait conservé les noms des sous-clans des Niḩad, un clan des Hुanéens (Hुanûm Niḩad). Il s'agit d'une liste divisée en deux colonnes. Dans la colonne de gauche figurent des noms uniques à Mari ou très rares et dans la colonne de droite sont enregistrés les noms des gens qui appartiennent à ces sous-clans: Hुalan (huit noms), Hुimum (trois), Bīt-Nūri (six), Bït-Wa/Yadabahah (trois), Bït-Wa/Yaninum (sept), Kuli'i (trois), Bahdatum (quatre) et Bït?-Ba-X (un). Nous supposons que ces noms sont les noms des sousclans des Hanéens. Enfin, dans un document juridique (VIII.11), on parle d'un

317 Miscellanea Babylonica, p. 281; voir aussi D. Charpin et J.-M. Durand, RA LXXX, p. 163. 318 p. 282.

319 p. 282. 
champ de Bīt-Awin, dont une partie des membres habite la localité d'Appān. Or, Appān est un village des Hanéens; de ce fait on pourrait conclure que Bīt-Awin est un sous-clan des Hanéens.

Dans les archives de Mari, nous trouvons un clan ou une tribu qui porte le nom de Yamahammûm. Une lettre en particulier fait penser que les Yamahammûm eux aussi appartenaient aux Hanéens. ${ }^{320}$ Il s'agit d'une lettre de La'ûm, le vizir de Yasmah-Addu, adressée à son maître, où il écrit entre autres: "Les moutons des Hanéens et des muškēnum (l'ensemble de la population en dehors de la couche supérieure et des nomades) d'Ah-Purattim ont traversé (le fleuve) en direction des oueds. Onze bergers relevant de Samaš-mušallim et les Hanéens Yamaḩammûm ont tous traversé (le fleuve). Ils paissent (leurs moutons) à Dūr-Yasmah-Addu et même en aval (de la ville)" (V.81.5-12). L'alternance entre Hanûm (1. 5) et Hanûm Yamahammûm (1. 9) et la tournure Ḩanûm Yamahammûm elle-même pourraient prouver que Yamahammûm appartenaient aux Hanéens. L'appellation Hanûm Yamaḩammû apparaît aussi dans une lettre qui concerne le Suhum (XXVI.468.22). D'après cette lettre, il semble qu'ils habitaient la ville de Yamaḩammûm. Les Yamaḩammûm, à l'instar des clans des Hanéens, des BiniYamina et Bini-Sim'āl, sont eux aussi qualifiés par le terme gā'um (IV.1.15-17). Le nom Yamahammûm, sous la forme du gentilice Ya/um(a)hammû, désigne aussi une localité dans le district de Saggarātum, à l'instar des autres unités tribales qui sont elles aussi des noms géographiques dans le district de Saggarātum: Amnanûm, Niḩadû, Yamutbal, Yariḩum, Zarri-Amnān et Zarri-Rabbium. ${ }^{321} \mathrm{Il}$ nous semble possible d'imaginer qu'à l'origine les Yamaḩammûm faisaient partie des Hanéens. Par la suite ils les auraient quittés pour devenir une tribu indépendante pouvant même rejoindre les Bini-Yamina, ${ }^{322}$ comme nous l'apprend une lettre écrite par le gouverneur d'un district (peut-être Saggarātum) à son seigneur:

320 J.T. Luke, Pastoralism, pp. 149-151; H. Klengel, Benjaminiten und Hanäer, pp. 211-212 et Ph. Talon, Miscellanea Babylonica, p. 283.

${ }^{321}$ VIII.67.4' (cfr. 1. 7' Nihadî [gén.]); XXI.59.22 (cfr. 1. 12 Zarri-Amnān); XXIII.69.9 (cfr. 1.5 Amnanî [gén.]; 1. 10 Niḩadî [gén.]); 427.IV.36' (cfr. 1. 37’ Niḥadî [gén.]); M.7450+.XII (cfr. Zarri-Amnăn, Nihadû); TRUP V.14 (cfr. 1.16 Niḥadû, VI.12 Yamutbal).

322 Voir Kupper, Nomades , pp. 20-21, 74. 
"Autre affaire. Au sujet des Bini-(ou: membres de la tribu) Yamaḩammûm, dont mon seigneur m'a écrit, depuis le jour où mon seigneur a frappé dans les campements des Bini-Yamina, ils (les Yamahammûm) sont partis. Il n'y en a plus aucun dans mon district. Le district va bien" (A.876.8'-14'). Jusqu'à maintenant nous avons constaté que la tribu Ḩanûm était divisée en huit ou neuf clans (Amurrum, Ibalahum, Naḩanum, Niḩad, Sibum, Yabasu, Yakallit, [Yamaḩammûm] et Yer'u). Un cas ébranle cependant quelque peu ce tableau homogène, il s'agit de la fameuse lettre de l'ordalie A.1251. Les habitants de la ville de Šnāā doivent déclarer à propos de la ville de Sunhum: "Et dep[uis] lo[n]gtemps elle avait vraiment été remise e[n] partage (à) aux $Y[a]-b[a]-s i-i m[\mathrm{k}] \mathrm{i} "$ (1. 25). Et leurs adversaires les habitants d'Apum eux doivent déclarer à propos de la même ville: "[Et d]epuis lon[gte]mps elle avait vraiment été remise [e]n partage (à) aux ḢA-

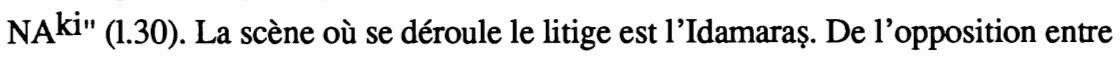
Yabasu et Hanûm on doit conclure que dans cette région, à un moment donné, les autres clans hanéens se sont opposés au clan Yabasu. Pour un phénomène semblable il suffit de se référer au livre des Juges chapitre 20, où est racontée la guerre entre tous les Fils d'Israël et la tribu Benjamin.

La plus petite unité tribale des Hanéens, le bït abim, apparaît une fois dans une lettre de Samsi-Addu à Yasmah-Addu, où il lui ordonne de choisir des Hुanéens pour la garde de son palais. Une moitié de ces Hanéens doivent être des gens riches, qui seront entretenus dans "les maisons de leurs pères" (II.1.21-23).

Les Hanéens se considèrent comme frères (A.1191.7, 11-12) et les membres d'un groupe hanéen qui stationnait dans le district de Qattunān pour le garder, s'appelaient entre eux "compagnons" tappū (XXVI.140.33). ${ }^{323}$ Enfin, les Ḩanéens considèrent aussi les Numhāa comme leurs frères (XXVI.358.6').

\section{Bini-Yamina}

Les Bini-Yamina, ${ }^{324}$ comme les Hanéens et les Sutûm, étaient eux aussi une con-

\footnotetext{
${ }^{323}$ Voir XXVI/1, p. 305, n. d.

${ }^{324}$ Les différentes façons d'écrire le nom sont enregistrées dans XVI/1, p. 39. Le second élément Yamina est toujours écrit syllabiquement, tandis que le premier élément est presque toujours écrit par l'idéogramme DUMU. Pour sa lecture trois possibilités sont proposées: 1. Lire cet idéogram-
} 
fédération tribale. Les Bini-Yamina comptaient cinq tribus. ${ }^{325}$ Dans la grande inscription de Yahdun-Lim on trouve trois de leur tribus qui sont identifiées explicitement comme appartenant aux Bini-Yamina: Uprapûm, Amnanûm et Rabbûm (M.2802.III.3-21). A ces trois tribus on peut ajouter une quatrième, les Yahrurûm, qui apparaît ensemble avec deux tribus mentionnées dans la grande inscription de Yahdun-Lim, les Uprapûm et les Amnanûm. Il s'agit d'une lettre de Kibri-Dagān, le gouverneur de Terqa, dans laquelle il identifie ces trois tribus comme des BiniYamina (III.50). Une cinquième tribu, les Yarihûm, apparaît dans une lettre de Samsi-Addu avec trois tribus dont nous venons de constater l'appartenance aux Bini-Yamina, les Uprapûm, les Yahrurûm et l'Amnanûm (I.42.29-32); d'où nous pouvons tirer la conclusion que les Yahrurûm appartenaient aussi aux BiniYamina. Les Yarihûm apparaissent avec les Uprapûm et les Yarihûum dans une autre lettre de Kibri-Dagān (XIII.105.5-10). Les tribus qui font partie de la confédération tribale Bini-Yamina se considèrent comme "frères" (I.6.9; 1.91+. $18^{\prime}$ ), appellation typique de toute une organisation tribale. Il faut aussi signaler que les noms de trois des tribus Bini-Yamina apparaissent en liaison avec le nom de la

me en accadien mãrum, selon l'avis de H. Tadmor, JNES XVII (1958), p. 130, n. 12. En effet nous trouvons une fois l'écriture syllabique Ma-ar (XI.43.20; J.-P. Materne, Miscellanea Babylonica, p. 229). 2. Lire l'idéogramme en Amurrite Bini, selon l'avis de Ph. Talon, Miscellanea Babylonica, p. 280, n. 13, qui s'appuie sur le nom propre Bi-ni-Ya-mi-na (XXII.328.III.16). Pour les noms propres qui sont des noms de tribus cfr. J.T. Luke, Pastoralism, p. 92, n. 19. 3. Lire tout simplement Yamina selon l'avis de I.J. Gelb, JCS XV (1961), p. 38. En effet nous avons une fois la forme l[ú Y]a-mi-na (XXI.339.7). Nous avons opté pour le nom devenu classique: Bini-Yamina, qui rappelle le nom de la tribu biblique Ben-Yamin, et par analogie avec le nom Bene-Qedem "les fils de l'est" (Gen. 29,1; Jug. 6,3).

${ }^{325}$ Voir J.T. Luke, Pastoralism, pp. 64, 67-68. Nous n'acceptons pas l'avis de M.B. Rowton (JNES XXXVI [1977], p. 189) selon lequel il y a une différence entre les Bini-Yamina et ces tribus. 
localité Zarri: Zarri-Amnān, Zarri-Rabbium (XVI/1, p. 41) et Zarri-Yarihûum (XXII.264.17'). Une preuve définitive de l'appartenance de toutes les cinq tribus aux Bini-Yamina se trouve dans deux documents économiques (XXIII.428-429), dont nous parlerons par la suite (voir ci-dessous Chapitre V, § 1B). Il est intéressant et peut-être significatif de signaler que le nom général de la confédération Bini-Yamina n'apparaît que dans un petit nombre de documents administratifs et économiques, à la différence du nom général Hुanûm qui est d'un usage répandu dans ces documents.

\section{Bini-Sim'āa}

Dans les lettres de Mari, nous trouvons le nom d'une tribu Bini-Sim'āl ("les fils du nord"), la tribu dont Zimri-Lim était le roi, au dire de Hammurabi, roi de Babylone (XXVI.385.6'). D'après G. Dossin ${ }^{326}$ les Bini-Sim'āl sont les "frères" des BiniYamina ("les fils du sud"). Et en effet, dans une lettre de Samsi-Addu (I. 60) nous trouvons l'appellation DUMU-MEŠ-Si-ma-al ù Ya-mi-in (ll. 9-10), où le membre DUMU-MEŠ "fils" est lié aux deux noms: Sim'āl et Yamin, au lieu de se répéter devant le nom Yamin. Dans une autre lettre on trouve la phrase suivante: "Cette ville est Bini-Sim'ālite et (ou: ou) cette ville est Bini-Yaminite (ou: ?)" (A.3080.1921). Et dans une autre lettre encore on lit: "[Au sujet des Han]éens qui ont [trav]ersé sur la rive ultérieure, à propos desquels mon seigneur m'a écrit en ces termes: les Huanéens qui ont traversé, Bini-Sim'ālites (ou) Bini-Yaminites, quel est leur gā'um ?" (A.2560.4-8). Mais il faut noter qu'à l'époque de Zimri-Lim nous rencontrons des cas où les deux tribus sont de vrais antagonistes: les rois d'Idamaraş demandent à Sibkuna-Addu, le roi de Šudā (ville de l'Idamaraș): "Pourquoi t'es-tu hâté de conclure une alliance avec Zimri-Lim et les Bini-Sim'āl et de parvenir à un accord avec eux ?". Sibkuna-Addu leur répond que cette alliance va permettre aux rois d'Idamaraș de pénétrer dans le Zalmaqum et de razzier le pays des Bini-Yamina. Nous voyons donc d'une part les rois d'Idamaraș, représentés par Sibkuna-Addu, Zimri-Lim et les Bini-Sim'āl, et d'autre part les Bini-Yamina (A.1208). On trouve la même situation dans l'Ah-Purattim, où Yaqqim-Addu, le

${ }^{326}$ BENJ., p. 995. 
gouverneur de Saggarātum, écrit à Zimri-Lim, que les Bini-Yamina se préparent à razzier les villages des Bini-Sim'āl qui se trouvent dans l'Ah-Purattim (XIV. 84+). Dans un autre cas encore, on voit dans les paroles de l'extatique Hubatum un antagonisme entre Zimri-Lim et les Bini-Sim'āl d'un côté et les Bini-Yamina de l'autre (XXVI.200.5-20). (*)

Au dossier des relations Bini-Yamina - Bini-Sim'āl, il faut ajouter aussi un document économique (XXV.130) qui enregistre $1 / 3$ mine d'argent pour le rachat d'une "fille de Sim'āl" sortie du service de Mukannišum. Or, ce document date de l'année ZL 4', l'année de la libération de beaucoup de Bini-Yamina (cf. XXIII, pp. 476478). Le fait que ce document date du 12.I.ZL 4' tandis que le premier document des Bini-Yamina date du 17.V.ZL 4' n'exclut pas, bien entendu, la possibilité que des Bini-Sim'āl aient été faits prisonniers lors de la guerre de Zimri-Lim contre les Bini-Yamina en l'an $\mathrm{ZL} \mathrm{1'327}$

\section{Hanûm, Bini-Yamina et Bini-Sim'āl}

Nous avons vu ci-dessus ( $§ 1$ ) les tournures "Hanûm Yamahammûm" et "Hanûm Nihad". Plus loin ( $\$ 5$ ) nous voyons la tournure "Sutûm Miḩalizayû". Dans les trois cas, le second nom d'une unité tribale est une sous-fraction du premier nom: Yamaḩammûm et Nihad sont deux clans des Hanéens et Miḩalizayû est un clan des Sutûm. Mais d'autre part nous trouvons (XXVI.62.7) le couple "Numhāā Yamutbal", et il est évident, nous semble-t-il, qu'il s'agit de noms de deux tribus ("Numhā̄" et "Yamutbal", VI.42.20; X.157.10). A la lumière de ces exemples il faut examiner les cas suivants: 1. Zimri-Lim donne l'ordre à Habduma-Dagān de "détruire les parcs à moutons et les ovins des Hanéens Bini-Yamina et de faire passer le Balīh (à ceux-ci)" (A.1086). 2. Dans une lettre nous lisons: "Là-bas, les Hanéens Bini-Sim'āl sont rassemblés par-devant mon seigneur. Qu'ici les Hanéens Bini-Yamina se rassemblent !" (A.556.40-41). 3. Ikšud-appašu écrit à Yasmah-Addu: "[Mon Seigneur] m’a envoyé po[ur réu]nir les Hanéens Bini-

${ }^{327}$ D. Charpin et J.-M. Durand écrivent au sujet des Bini-Sim'ăl dans RA LXXX, p. 150: "le fichier de Paris dans son état présent ... offre plus d'une soixantaine de références supplémentaires aux Benê-Sim'al". 
Yamina" (M.8512.5-6). La conclusion à tirer de ces exemples est sans doute que les Bini-Yamina et les Bini-Sim'āl sont des sous-fractions des Hanéens, car il est peu probable que nous ayons affaire à "Ḥanéens et Bini-Yamina" ou "Huanéens et Bini-Sim'āl". Nous devons cette conception nouvelle à D. Charpin et à J.-M. Durand. ${ }^{328}$ Ils ont apporté d'autres exemples pour étayer leur thèse, citons les cas suivants: 4. Sîn-tiri, le gouverneur de Harrān, écrit à Yasmah-Addu une lettre dont nous avons déjà cité le passage suivant: "[Au sujet des Huan]éens qui ont [trav]ersé sur la rive ultérieure, à propos desquels mon seigneur m'a écrit en ces termes: les Hanéens qui ont traversé, Bini-Sim'ālites (ou) Bini-Yaminites, quel est leur gā'um ?" (A.2560.4-8). 5. Yasmah-Addu, le roi de la tribu bini-yaminite Yarihûm, écrit à Zimri-Lim: "Précédemment déjà j’ai écrit à mon seigneur au sujet des troupes et mon seigneur m'a écrit: monte sur les bateaux de Lahun-Dagān et viens. Voilà ce que mon seigneur m'a écrit. Maintenant, je suis disposé à partir, mais les hommes de Yarihûm, les sugāgū des Hुanéens sont arrivés et m'ont retenu. Autre chose: les sugāgū des Bini-Yaminites se sont rassemblés à Zalpah, puis ils se sont rendus à Ahunā, et Șū[rā-Ḩammu et Yarīm-Lim] ont siégé et à [Șūrā-Huammu et] Y]ar[im-L]im ils ont parlé. Les s[ugāg]ū des Huanéens se sont levés et à Yar[īm-L]im et Șūra-Hămmu ont dit: [al]lez vers Zimri-Lim et réclamez nos villes. [S]i Lahunun-Dagãn [ne] veut pas aller, ou nous le tuerons, ou bien, luimême, nous le chasserons de son trône. Voilà ce que disent les sugāg[ū] des Bini-Yaminites. Or ça, [ma]intenant Yarīm-Lim, Șurā-Ḩammu et les sugāgū $\mathrm{v}$ [on]t chez mon seigneur. Tout ce qu'ils réclament, que mon seigneur ne leur refuse pas; et moi, j'arriverai après eux!" (II. 53). On constate l'alternance entre "Les sugāgu des Hanéens ... ont dit" (1l. 17-19) et "Voilà ce que disent les sugāgū des Bini-Yaminites" (11.25-26) - dans les deux cas on se réfère au même groupe. 6. J.-M. Durand ajoute un autre exemple de ces "Hanéens-Bini-Yaminites". 329 Après la conquête de la ville de Mišlān (XXVI.282.14-15) on conseille à ZimriLim: "Il faut conduire à la frontière deux Hुanéens vivants et, à la frontière, les mutiler. Ils doivent aller, vivants, aux Bini-Yamina pour dire que mon Seigneur a pris de force la ville de Mišlān" (1l. 19-25). Que vont faire ici ces deux Ḩanéens,

328'Fils de Sim'al'': les origines tribales des rois de Mari, RA LXXX [1986], pp. 141-183.

${ }^{329} \mathrm{XXVI} / 1$, p. 583 , n. e. 
car la ville de Mišlān est une ville bini-yaminite ? Et s'il s'agit en effet des "Hanéens-Bini-Yaminites" pourquoi distingue-t-on entre "deux Hanéens" (1. 19) et Bini-Yamina (1. 22) ? (*) Peut-on accepter pour toute la documentation de Mari la nouvelle compréhension de D. Charpin et J.-M. Durand ? Nous pensons que la réponse à cette question est négative, car de toute l'énorme documentation sur les nomades à Mari on n'a relevé que quelques exemples sûrs. A la rigueur on pourrait supposer, tenant compte de la thèse de D. Charpin et J.-M. Durand, qu'à l'origine, on aurait eu affaire à un seul groupe ethnique, celui des Hanéens, qui se serait scindé en deux à un moment donné: le groupe du nord, dans le triangle du Habur (Bini-Sim'āl) et le groupe du sud, sur le moyen-Euphrate (Bini-Yamina), comme les Mawali qui se divisent en Semāliyīn et Qibiliyīn, 330 " les gens du nord et les gens du sud", appellations qui ne représentent plus de réalité géographique. D'autre part, on pourrait aussi envisager la possibilité que dans certains cas Hanûm est employé comme un nom général signifiant "nomades". On trouve maint exemple de nom ethnique devenu nom général. Citons d'abord un exemple tiré de la première campagne de Sennachérib où le nom Aramu est employé d'abord comme nom général (I.39), puis comme nom ethnique (I.49). Le second exemple est biblique: Ismaélites dans Ps 83:7 est un nom ethnique tandis que dans Jug 8:24 il est employé comme nom général. ${ }^{331}$ Il semblerait donc, sans certitude absolue, que la dernière hypothèse convienne dans la plupart des cas.

\section{Sutûm}

Les Sutûm comme les Hanéens et les Bini-Yamina étaient eux aussi une confédération tribale. Nous connaissons les noms de trois de ces tribus: Dans un document qui enregistre les taxes que les Sutûm ont prélevées sur leurs troupeaux, étaient mentionnés les noms de trois tribus (IX.244). Aujourd'hui sont conservés seulement un nom complet et un nom partiellement restitué: Almutû, Miḩal[izayû]. Almutû apparaît, avec une autre tribu, dans une lettre de Yaqqim-Addu, le

330 Voir M.P. von Oppenheim, Die Beduinen, p. 311.

${ }^{331}$ Cfr. J.-R. Kupper, Nomades, pp. 73-74, 138-139, 143; M. Anbar, Biblica XLIX (1968), pp. 230-232. 
gouverneur de Saggarātum: "Ces hommes, qu'on les vende aux Sutûm (les plus) éloignés, aux Yaḩmamû ou aux Almutû" (XIV.78.8-10, 13). Quant aux Miḩalizayû, on les trouve dans une lettre de Bahdi-Lim, le gouverneur de Mari: "Les Sutûm Miḩalizayû ont dé[couvert] de l'argent, un trésor, au (Mont) Bisir" (VI. 44.5-6). Enfin D. Charpin et J.-M. Durand citent les noms de deux clans des Sutûm: Barḩalānum et Buli'ila (A.3592). Voici enfin les noms des tribus des Sutûm dans l'ordre alphabétique: Almutû, Barhạaānum, Buli'ila Miḩalizayû et Yahmamû.

\section{L'analyse des noms propres des membres des trois grandes tribus}

Dans l'analyse des noms, nous avons fait de notre mieux pour éviter les cas où le même membre d'une tribu, qui apparaît dans deux ou même plusieurs documents, soit compté pour plusieurs personnes différentes. Ceci risque bien entendu de diminuer un peu le nombre des personnes citées pour chaque tribu, puisque là où un nom qui apparaît plusieurs fois dans les documents, sans une qualification qui puisse l'identifier - à part l'appartenance à une certaine tribu - a été cité par nous seulement une fois, il peut très bien s'agir en fait de plusieurs personnes de la même tribu. Les noms qui ne sont pas complets à cause du mauvais état des tablettes n'ont pas été inclus dans le calcul, sauf si la partie conservée du nom contient un élément qui permette son classement. Dans le cas de Hुanûm, et surtout de Bini-Yamina, nous avons aussi pris en considération les habitants des localités dont on sait qu'elles appartenaient aux Hanéens ou aux Bini-Yamina. Voici les nombres des membres des tribus que nous avons comptés: 639 Ḩanéens, dont 85 noms sont incomplets, 483 Bini-Yaminites, dont 65 noms sont incomplets, 44 Sutéens dont 2 noms sont incomplets, 2 Bini-Sim'ālites portant des noms amurrites, 8 Yamutbaléens, dont 5 portent des noms amurrites et 3, des noms incomplets, 3 Numahéens dont deux portent des noms amurrites, la troisième un nom d'origine inconnue; enfin un Ya'ilānéen portant un nom amurrite.

L'analyse des noms propres nous montre que les Hanéens sont les moins accadianisés par rapport aux Bini-Yamina et même aux Sutéens. 
Am. $=$ Amurrite, Ac. $=$ Accadien, Sum. $=$ Sumérien, H. $=$ Hurrite, $?=$ d'origine inconnue, $\mathrm{h}=$ Hanûm, by $=$ Bini-Yamina, $\mathrm{s}=$ Sutûm, $\mathrm{g}=$ nom géographique, $\mathrm{e}=$ nom ethnique.

$\begin{array}{llllllll} & \text { he } & \text { hg } & \text { heg } & \text { bye } & \text { byg } & \text { byeg } & \text { s } \\ \text { Am } & 86.41 \% & 75 \% & 84.6 \% & 59.34 \% & 71.18 \% & 67.3 \% & 71.4 \% \\ \text { Ac } & 7 \% & 11.9 \% & 7.7 \% & 22.76 \% & 17.96 \% & 19.2 \% & 16.6 \% \\ \text { Am./Ac. } & 4 \% & 7.14 \% & 4.5 \% & 4.8 \% & 6.44 \% & 5.9 \% & 9.5 \% \\ \text { S. } & & & & 0.81 \% & & 0.23 \% & \\ \text { H. } & 0.2 \% & & 0.18 \% & & & & \\ ? & 2.33 \% & 5.95 \% & 2.8 \% & 12.19 \% & 4.4 \% & 6.6 \% & 2.3 \%\end{array}$

Il est intéressant de comparer ces données avec l'analyse des noms propres qui apparaissent dans les textes de Mari publiés jusqu'en 1981 (cfr. ci-dessous Chap. VIII). Nous possédons les noms de 1190 membres des tribus amurrites, dont 155 sont incomplets, et en conséquence ne sont pas inclus dans le calcul:

$\begin{array}{ll}\text { noms propres à Mari } & \text { noms propres de } \\ & \text { membres des tribus }\end{array}$

$\begin{array}{lll}\text { Am. } & 40.4 \% & 77.31 \% \\ \text { Ac. } & 33.6 \% & 12.75 \% \\ \text { Am./Ac. } & & 5.25 \% \\ \text { S. } & & 0.09 \% \\ \text { H. } & 9 \% & 0.09 \% \\ ? & 16 \% & 4.47 \\ \text { divers } & 1 \% & \end{array}$

Compte tenu du fait que dans le calcul de C. Rasmussen sont inclus aussi les membres des tribus amurrites connus à l'époque de son étude (1981), on peut affirmer que le pourcentage de noms amurrites des membres des tribus amurrites est le double de celui d'autres personnes qui figurent dans les textes de Mari. 


\section{Chapitre IV: La distribution géographique}

Dans le présent chapitre nous allons passer en revue tout ce que nous savons sur la présence des tribus amurrites dans le royaume de Mari et dans les pays voisins. La discussion s'organisera géographiquement et le résumé du chapitre sera arrangé tribu par tribu.

\section{Le district de Mari}

A. Ḩanûm - Dans une lettre que Baḩdi-Lim, le gouverneur de Mari, a envoyée à son maître Zimri-Lim, nous lisons qu'il a attendu cinq jours pour le rassemblement des Hanéens dans un endroit convenu. Or, les Hanéens, qui sont retournés à leurs villes depuis la steppe, n'ont pas consenti à se rassembler, en dépit du fait qu'il les a convoqués une fois, deux fois de leurs villes. Pour les stimuler à se rassembler à l'endroit convenu, Bahdi-Lim suggère à Zimri-Lim de les menacer par un acte dramatique: couper la tête d'un coupable et la faire circuler entre les villes des Ḩanéens "jusqu'à Ḩutnum et Appān" (II.48.5-20). Les sugāgū de Huutnum et d'Appān ont envoyé des moutons au Palais de Mari (VII.225.Rev.2', 4'; 226.4244). Appān est probablement la ville la plus éloignée parmi les villes des Hanéens dans le district de Mari. La ville apparaît comme une ville frontière dans une autre lettre, où on parle d'une installation des gardes pour empêcher le passage des Hanéens, ${ }^{306}$ probablement à la steppe. Les gardes seront installés "depuis Appān jusqu'à notre (de Mari) puits" (XXVI.41.4'-5'). ${ }^{307}$ Par un document juridique nous savons qu'à Appān vivaient des membres de Bīt-Awin (VIII.11.9-10). A une autre occasion, quand on veut rassembler les Hanéens, on lève des signaux lumineux dans la ville de Dīr, qui se trouve à une journée de marche au sud de Mari ${ }^{308}$ (SIGN., p. 183, n. 2). Il se peut que Dīr soit l'endroit où Bahdi-Lim

306.-R. Kupper, Nomades, p. 12, n. 6.

307J.-M. Durand traduit: "jusqu’à Ni'âtum-le-puits".

${ }^{308}$ M. Birot, RA LXVI (1972), pp. 135-136. 
attendait les Hanéens. Cette supposition pourrait être renforcée par le fait qu'à Dìr a eu lieu le recensement des Bini-Yamina avant leur départ pour la Babylonie (VI.30), et que des armées des Hanéens y sont passées à leur retour de la Babylonie (XIII.33.5-9). La ville de Dīr n'appartenait, nous semble-t-il, ni aux Hanéens ni aux Bini-Yamina, mais elle était un centre militaire à la frontière sud du district de Mari. Yatarum, fils de Tillabnû, un Hanéen qui vivait à Suqaqum, et deux Hanéens ont accompagné le roi d'Ašnakkum qui se rendait à Mari (III.46). ${ }^{309}$ On pourrait en conclure que Suqaqum était elle aussi une localité hanéenne. Enfin, des membres du clan Yamaḩammûm, qui servaient chez SamsiAddu, habitaient dans deux villages du district de Mari, à Harratum et à Amatum (IV.1).

Nous voyons que dans le district de Mari on trouve une très forte présence des Hanéens; pour cette raison il est possible que la désignation "pays des Hanéens" dans les inscriptions de Yahdun-Lim et Zimri-Lim ${ }^{310}$ se réfère au district de Mari $^{311}$ à l'instar du "pays d'Uprapûm", qui se réfêre au district de Samānum (voir ci-dessous § 2). La ville de Haman "le centre des Hanéens" (M.2802.III.28) se trouve, peut être, dans le district de Mari. ${ }^{312}$

B. Bini-Yamina - Au temps de Yasmah-Addu, on constate que parmi des gens de son district (I.20.12'; 42.15), celui de Mari, ${ }^{313}$ qui prennent part à l'expédition pour Qatanum, se trouvent six cents membres de quatre tribus des Bini-Yamina: les Uprapûm, les Yarihûm, les Yaḩrurûm et les Amnanûm (I.42.29-31). ${ }^{314}$ Les Bini-Yamina continuent à être présents dans le district de Mari aussi durant le règne de Zimri-Lim, comme nous l'apprennent les listes du recensement des Bini-

${ }^{309}$ Voir M. Anbar, N.A.B.U. 1988/32.

$310 \mathrm{XVI} / 1$, p. 13; D. Charpin et J.-M. Durand, RA LXXX, pp. 149-150.

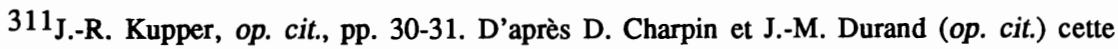
désignation se réfêre à l'Idamaraş.

312 J.-T. Luke, Pastoralism, p. 156.

313J.-R. Kupper, op. cit., p. 50.

314J.-R. Kupper, op. cit., p. 47 et J.-T.Luke, op. cit., p. 96, n. 70 ajoutent aussi I.6.6-21 comme preuve de la présence des Bini-Yamina dans le district de Mari. 
Yamina de l'année ZL 10' (XXIII.428-429): Zimri-Lim a envoyé une armée à Hammurabi dans l'année ZL 9'. Cette armée, qui était composée surtout de Hanéens, est retournée à Mari avant l'hiver de la même année, car Zimri-Lim a eu besoin d'elle pour ses campagnes en Idamaras.. A la place de cette armée, il envoie à Ḩammurabi au début de l'année ZL 10' une nouvelle armée qui inclut des supplétifs (warkûm) des Bini-Yamina. D'après les listes du recensement de ces Bini-Yamina, on constate que des Yahruréens habitaient dans les localités suivantes: Dunnum, Mišlān, Saadudum, Saa-ḥu/işurātim et Zarri, et des Yariḩéens habitaient dans Zurmahum. Zurmahum n'était pas la seule ville où habitaient les Yarihû̂m. Leur roi, Yasmah-Addu, mentionne des villes dans le district de Mari où se trouvent des gens de sa tribu (II.55.31). Parmi les Bini-Yamina qui ont été libérés par leurs parents durant l'année ZL 4', on trouve de nouveau des habitants de Mišlān, Ša-huu/iṣurātim et Zarri (cfr. XXIII, p. 481). Dans des documents de l'année ZL 2' est mentionné le butin de Mišlān (cfr. XXIII, p. 482). La ville de Mišlān figure parmi les localités qui ont levé des signaux lumineux au moment où l'employé de Zimri-Lim, Bannum, est arrivé à Zurubbān (SIGN., p. 178, 1. 13). Les sugāgū de Dunnum et de Mišlān ont envoyé des moutons au Palais de Mari (VII.226.39; XXI.56.9). Mišlān dont le roi fut Yagih-Addu, ${ }^{315}$ était une ville entourée d'un rempart, qui fut détruit par Zimri-Lim dans l'année ZL 1' (N.AN., p. 55, no. 5): "Depuis que mon Seigneur a pris la ville de Mišlān" (XXVI.282.1415). Les lettres des serviteurs de Yagih-Addu nous apprennent elles aussi que la ville était une ville fortifiée avec sa muraille et ses portes. ${ }^{316}$ Les autres localités n'étaient selon toute vraisemblance que de simples villages. Dans une des lettres du majordome du Palais de Mari, Yasīm-Sūmû, il est question "de champs du territoire des Bini-Yamina" (XIII.39.4, 7); il semble qu'il s'agisse d'un territoire du district de Mari où habitent des Bini-Yamina. ${ }^{317}$

C. Bini-Sim'āl - Yaḩdun-Lim n'était pas seulement considéré comme "roi de Mari et du pays des Ḩanéens" il se peut qu'il ait porté aussi le titre de "roi de Mari

\footnotetext{
315 D. Charpin et J.-M. Durand, RA LXXX, p. 145 (prince); XXVI/1, pp. 214(d), 337 .

316XXVI.168.34; 169.11; 170.24'-25'; 171.20', 25'; 172.15-16, 41-42.

${ }^{317}$ M. Birot, Syria XLI (1964), p. 49, et voir aussi M.8795.
} 


\section{Les tribus amurrites de Mari}

et du pays des Bini-Sim['āl] (DUMU Si-im- [a-aI])" au dire de la légende du sceau d'une de ses filles. ${ }^{318}$ Peut-être cette désignation se référait-elle au district de Mari à l'instar de la désignation "pays des Hanéens".

\section{Le district de Terqa}

A. Hanûm - Dans toutes les lettres de Kibri-Dagān, le gouverneur de Terqa, on ne trouve même pas un témoignage de la présence des villes des Hanéens dans son district. Pour cette raison Kibri-Dagān était forcé d'aller en Idamaraș pour ramener de là-bas trois mille Hanéens (III.14; XIII.102; 103). Par contre, nous savons qu'un nawûm (troupeaux et leurs bergers) des Hanéens paissait dans son district (III.15).

B. Bini-Yamina - Il semble que la présence des Bini-Yamina dans le district de Terqa ait été plus marquante que dans les districts de Mari et de Saggarātum (voir ci-dessous § 3B). ${ }^{319}\left(^{*}\right)$ Plusieurs lettres de Kibri-Dagān traitent de "villes des Bini-Yamina" de son district. ${ }^{320}\left(^{*}\right)$ Tous les habitants de ces localités avaient l'habitude de transhumer en amont de l'Euphrate: III.58.5-10.321 Dans les listes de recensement des Bini-Yamina apparaissent les localités suivantes du district de Terqa: Dašrān où habitaient les Yaḩrưûm, Amiyān et Damiqān où habitaient les Yarihûum, Kirêtum et Kulhitum où habitaient les Amnanûm, Dimtān où habitaient les Rabbûm, et Ilum-Muluk, Raqqum, Rasāyum et Samānum où habitaient les Uprapûm. Dans les listes des Bini-Yamina libérés ou attribués à d'autres services (XXI.412; XXIII, p. 481; M.7168) apparaissent de nouveau Dašrān, Dimtān, Ilum-Muluk, Raqqum, Rasāyum et Samānum, et en plus les localités suivantes:

${ }^{318}$ Voir N. Özgüç dans Ancient Art in Seals, E. Porada (éd.), Princeton, 1980, pp. 61-62; photo fig. 3 a, b. Pour la lecture DUMU Si-im- [a-al], cfr. D. Charpin et J.-M. Durand, RA LXXX, p. 152 et $n .56$.

${ }^{319}$ Cfr. XXIII, p. 361.

${ }^{320}$ II.92.5, 12, 15; III.12.19, 25; 16.5, 13, 22; 21.5, 13; 58.6; 70.6', et cfr. aussi la lettre XXVI.450.6-8.

${ }^{321}$ Cfr. aussi II.92.15-16, 25-26; III.12.18-20, $25-26$ 
Dabiš, Gadlum et Šalabatum. Les sugāgū de Dabiš, Damiqān, Dašrān, Dimtēn (= Dimtān), Ilum-Muluk, Kirêtum, Kulḩitum, Raqqum et Rasêm (= Rasāyum) ont envoyé des moutons au Palais de Mari (VII.201.1'; 225.2, 3; XXI.56.11-18). Sur quelques-unes de ces localités on peut rassembler les renseignements suivants:

a) Amiyān, Damiqān et Dumtān (= Dimtān): Le sugāgum de Dumtān refuse à l'appel de Zimri-Lim de rassembler ses gens dans les villes fortes (III.38.15-23). Les quatre localités: Amiyān, Damiqān, Dumtān et $[\mathrm{X}]$ refusent de prendre part au recensement que Kibri-Dagān a effectué (III.19.5-22; 20).

b) Dašrān: Un habitant de la ville du nom de Irra-Malik était un extatique muhhûm (M.9921.III).

c) Raqqum: Dans l'année ZL 2' est mentionné le butin de la ville de Raqqum (cfr. XXIII, p. 482). (*)

d) Samānum: La ville de Samānum était au temps de Yaḩdun-Lim une villeroyaume (cfr. Josh 10:2) aux mains du roi des Uprapûm, et un centre des BiniYamina où ceux-ci se rassemblaient pour faire la guerre à Yahdun-Lim (M. 2802.III.16-18). ${ }^{322} \mathrm{Au}$ début du règne de Zimri-Lim, Samānum était le siège de

322 Dans cette grande inscription de Yahdun-Lim (M. 2802.III) nous trouvons les phrases suivantes: "la ville de Samånum ummat Bini-Yamina (TUR-mi-im)", III. 16-17 et "la ville de H̆amån ummat H̦anûm ", III. 28. A. Malamat (UF XI [1979], pp. 527-536) compare cet umma$t$ (um) avec l'hébreu 'umma, "tribu". D'après $A H w$ (p. 1414b[1e]) le mot signifie ici "Hauptmacht ". Mais le fait que le mot est en apposition avec un nom de ville nous amène à accepter la traduction de A.L. Oppenheim (ANET S, col. 120 [556]) par "centre". A. Malamat à la suite de W. von Soden (Or. XXI [1953], p. 205, no. 74.22, 29 et 1414b[1c]) pense que dans les autres cas où apparaît le mot ummatum il s'agit aussi d'une unité tribale. A notre avis ce mot, qui à la différence de gå'um et de hibrum, est un mot accadien n'a aucun rapport avec l'organisation tribale. Dans des documents de Mari de l'époque assyrienne et de Qałłară on trouve la locution ummat persim "l'ummatum de la section" (ARMT IV.74.22, 29; OBTR 244.IV.21, V.2; 245 [passim]; 254.7; 263.3; 323.17). Comparons les deux listes de payements suivantes:

a. "Les capitaines, les centurions, les grands des sections, les sugãgū et l'ummatum des sections" (IV.74.20-22).

b. "les capitaines, les sugăgū, le forgeron, le héraut et les centurions (VII.215.34-35). 
Samsi-Addu, le bini-yaminite. ${ }^{323}$ Dans une lettre Kibri-Dagān annonce à son maître que dans les villes des Bini-Yamina de son district, nombreuses sont les femmes dont les maris se sont enfuis de leurs maisons pour joindre l'ennemi (sans doute leurs frères Bini-Yamina). Ces hommes entraient pendant la nuit en petits groupes chez leurs femmes et partaient en emportant avec eux des renseignements sur Kibri-Dagān. Un de ces groupes de maris se dirigeait vers Samānum (III. 16). Les villes de Samānum et Ilum-Muluk, du district de Terqa, et la ville de Mišlān, du district de Mari, ont levé des signaux lumineux, et toutes les villes des BiniYamina du district de Terqa leur ont répondu également par des signaux lumineux (SIGN., p. 178). Samānum, comme Mišlān du district de Mari, était entourée d'un rempart qui a été détruit une première fois par Yahdun-Lim (M.2802.III.25) et une seconde fois par Zimri-Lim durant l'année ZL1' (N. AN., p. 55, no. 5). L'employé Hali-ḩadun conseille à Zimri-Lim de donner aux Hanéens, qui descendront dans l'Ah-Purattim, un des villages des Bini-Yamina (BENJ., p. 984d, e). L'Ah-Purattim, qui signifie littéralement le "bord de l'Euphrate", se rapporte à la vallée de l'Euphrate entre Mari et Saggarātum (XIV. 84+.37-46; A.2231.5-6, 5'-6'). Or, comme dans sa lettre Hali-hadun parle d'une région où on ne trouve pas de localités des Hanéens, mais des localités des Bini-Yamina, on peut conclure qu'il s'agit ici du district de Terqa, où des Hanéens faisaient paître leurs troupeaux (III.15). Les troupeaux des Bini-Yamina entrent dans le district et cela y provoque de l'inquiétude (II.90). Et enfin, dans une lettre dont le nom de

On constate par cette comparaison que l' "ummatum des sections" dans une liste correspond aux forgeron et héraut danș l'autre. Comparons maintenant un autre couple:

a. "69 gens de la section de Awan" (XXII.40.II'.16').

b. "199 gens de l'ummatum de la section de Baliya" (OBTR 244.V.1-2).

On constate ici que "gens" et ummatum s'interchangent. L'ummatum signifie, semble-t-il, la masse de gens qui forme la section, à l'instar de la masse (ummatum) d'huile qui apparaît au cours de l'acte divinatoire par l'huile ( $A H w$, p. 1415a [6]). Un terme à part est le tuppi ummatim qu'on trouve une fois à Mari (VI.77.18) et plusieurs fois en Babylonie ( $A H w$, p. 1414b; D. Charpin, CR XXX $R A I$, pp. 121-140).

${ }^{323} \mathrm{XXVI} / 1$, p. 340, n. 26. 
l'expéditeur manque, mais il est très probable qu'il s'agit de Kibri-Dagān, on lit que les Bini-Yamina se révoltent (SIGN., p. 181a). $\left({ }^{*}\right)$

C. Bini-Sim'āl - On a mêlé des déportés de la ville de Rapiqum qui se trouvaient dans Terqa à des membres de la tribu Bini-Sim'āl pour garder la ville (XXVI.144.18'-21').

D. Sutûm - Les Sutûm arrivent dans le district de Terqa au cours de leurs transhumances. Kibri-Dagãn écrit à Zimri-Lim que les Sutûm campent à trois doubles-heures $(=30 \mathrm{~km})$ en amont de Terqa. La présence de ces Sutûm pourrait signifier des troubles. Pour cette raison, Kibri-Dagān calme Zimri-Lim en l'informant: "et ils viennent constamment, se rencontrent avec moi, puis s'en retournent" (III.12.10-15).

E. Numḩā et Yamutbal - Les Numḩā et Yamutbal arrivent eux aussi au district de Terqa. Le fonctionnaire Asqudum informe Zimri-Lim: "Je suis arrivé au district de Hišamta et Terqa. Numḩā, Yamutbal avec garçons, filles (ou: toute la population), esclaves, servantes, boeufs et ânes - ils sont innombrables (pānam u bābam

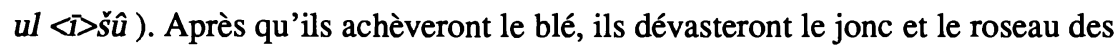
bords de l'Euphrate" (XXVI.62.5-13). Or, il est intéressant de noter que la présence des sauterelles est décrite par la même expression: "Les sauterelles (erbum) sont innombrables (pānam u bābam ul ǐsûu à Dīr" (A.3872+.4-6). La scène décrite dans ces passages rappelle la montée des Madiân, Amaleq et Bene-Qedem sur Israël décrite dans le récit de Gédéon: "En effet, ils montaient, eux et leurs troupeaux, avec leurs tentes, arrivaient aussi nombreux que des sauterelles (' $r b h$ ) eux et leurs chameaux étaient innombrables - et ils entraient dans le pays pour le ravager" (Jug VI:5).

\section{Le district de Saggarātum}

A. Ḩanûm - Dans une lettre que Yaqqim-Addu, le gouverneur de Saggarātum, écrit à Zimri-Lim, nous lisons qu'il a fait partir tous les Hुanéens des villes qui se trouvent dans son district (XIV.80.18-20). Sumḩu-rabi, le gouverneur de Tuttul, 
écrit que "les Hुanéens, depuis Halabit jusqu'à Lasqum ${ }^{324}$ se sont établis, séparément" (A.4018.4'-6'). Dans le district se trouve une ville qui porte le nom du sous-clan Niḩad - Niḩadû (XXIII, p. 592b). Les troupeaux des Yamaḩammûm paissaient dans la région de Dūr-Yasmaḩ-Addu (V.81). Des gens de Yamahammûm apparaissent dans les listes des habitants du district (XXIII, p. 595a; TRUP.V.14). Dans une liste, que nous supposons être une liste des sugāgū de Saggarātum, on trouve le nom de Zakira-Hammu (VII.213.4). Or ce nom était aussi celui d'un sugāgum du clan Nahan (VII.227.6') et d'un sugāgum du clan Ibalahu ${ }^{325}$ d'où on peut tirer la conclusion que soit le clan Naḩan soit le clan Ibalahu séjournait dans le district de Saggarātum. Les habitants de deux villes qui portent les noms de clans ḩanéens: Niḩadûm et Yamaḩammûm, avec les habitants des autres villes se rassemblent dans Dūr-Yahdun-Lim: "Les villes aux alentours de Dūr-Yaḥdun-Lim: Huurrān, Naḩadûm (= Niḩadûm), Yumḩammûm, Narâ se sont rassemblées à l'intérieur de Dūr-Yahdun-Lim" (A.3550.11-15).

B. Bini-Yamina - Les Bini-Yamina ont subi deux défaites dans le district de Saggarātum. La première de la main de Yasmaḩ-Addu ${ }^{326}$ environ en 1779/1778, et la seconde par Zimri-Lim ${ }^{327}$ en 1773/1772. On sait aussi qu'ils ont attaqué Dūr-Yahdun-Lim (XXVI.121.9-15), et ont subi là-bas une défaite devant ZimriLim (XXIV.170+258.4'-5'). Et parce qu'ils ont constitué un danger pour le cortège de Sibtum, on évita de passer par Ganibatum (XXVI.16.20-22). Dans le district de Saggarātum on trouve des localités habitées par les Bini-Yamina (XIV.89.11'). Ces localités, appelées villes (ālānu), étaient en vérité des villages (kaprātum) comme nous l'apprend une lettre de Yaqqim-Addu (II.102), qui annonce à Zimri-Lim que tous les habitants des localités (ālānu, 1. 7, kaprātum, 1. 9) ont transhumé en amont de l'Euphrate. Cette transhumance est rappelée dans une autre lettre de Yaqqim-Addu (II.92.14-29). La descente des Bini-Yamina et

${ }^{324}$ Dans le secteur de Halébiyé d'après J.-M. Durand, Miscellanea Babylonica, p. 81; XXVI/1, pp. 125-127.

${ }^{325} \mathrm{Ph}$. Talon, Miscellanea Babyonica, p. 281.

${ }^{326}$ M. Birot, M.A.R.I. IV, p. 226.

${ }^{327}$ N.AN., p. 55, no. 7; XXI.128.10-13. 
leur installation dans Saggarātum est rappelée dans un passage d'une lettre de Sammetar (XXVI.199.32-35). ${ }^{328}$ Nous connaissons les noms de quelques-unes des ces localités. Dans les listes du recensement des Bini-Yamina apparaissent les localités suivantes: Dabiš où habitaient les Yaḩrurûm, Zapat où se trouvaient les Yarihûm, Sahriya et Zarri-Amnān où vivaient les Amnanûm, et Zarri-Rabbiyum où habitaient les Rabbûm. Dabiš apparaît aussi dans les listes des Bini-Yamina libérés (cfr. XXIII, p. 481; M.7168). Le sugāgum de Dabiš a envoyé des moutons au Palais de Mari (VII.225.3). Dans une liste de rendements en orge du district, on trouve la localité Amnanûm (XXIII.69.5). Dans la ville de Saḩrû (= Saḩriya) habitaient des Amnanûm (XIV.64.10'-11'; 89.4-5). Șūra-ḩammu, le roi des Amnanûm, visitait les villes de Saḩrû et Zarri-Amnān où habitaient les membres de sa tribu (II.105.9-11; XIV.83.7-8 [cfr. aussi VIII.67.3-5]). En plus de ces localités, on constate que Lasqum, une région de la haute steppe, était le dīra (le terme arabe pour désigner un pâturage réservé à une tribu ${ }^{329}$ ) des BiniYamina (XIV.85.6-8; BENJ., p. 986, n. 1). Une fois, des troupeaux des Hanéens ont envahi cette région (XIV.81.4-16). On apprend par une lettre du gouverneur de Qattunān datant du début du règne de Zimri-Lim, que Yagih̆-Addu, le roi BiniYaminite, et 1000 de ses hommes ont conquis la ville de Manuhatān, et que tout le campement des Bini-Yamina s'est installé à Lasqum et dans la ville même. ${ }^{330}$ Enfin, une lettre nous fait savoir que "Les Yahruréens, après avoir passé quelques jours dans la forêt, ont traversé (l'Euphrate) à Halabit" (A.1176).

C. Yamutbal - Un document atteste la présence des gens de la tribu Yamutbal qui séjournaient dans le district de Saggarātum (TRUP.V.9, VI.12). Et ce peut être à cette région que doit être rattaché le "district de Yamutbal" mentionné dans une lettre (A.915.7').

\footnotetext{
${ }^{328}$ Voir aussi XXVI.39.30, 44-46; A.1281.

${ }^{329}$ E. Marx, American Anthropologist LXXIX (1977), p. 351.

${ }^{330} \mathrm{XXVI} / 1$, p. 141, n. 22 . Nous préférons traduire ici epešum par "conquérir" et non par "fortifier". En outre, si le verbe accadien qui se cache derrière "s'installer" est bien sakănum, on notera un autre cas où le verbe s'associe avec le nawûm.
} 


\section{L'Ab-Purattim}

Ah-Purattim signifie littéralement "Bords-de-l'Euphrate", mais il semble que, outre le fait qu'il s'agit d'un terme géographique pour désigner la vallée de l'Euphrate, il désigne aussi la région qui s'étend dans la vallée de l'Euphrate entre Saggarātum et Mari (XIV.84+.4'-13'). 331

A. Hanûm - Le fonctionnaire Hali-ḩadun conseille à Zimri-Lim: "Si c'est la volonté de mon seigneur, que les Hanéens descendent ici-même, sur l'Ah-Purattim, et qu'on leur donne un village parmi les villages des Bini-Yamina" (BENJ., p. $984 d$ et cfr. e). Du fait qu'il s'agit apparemment d'une région où on ne trouve pas de villes des Hanéens, mais seulement des Bini-Yamina, on peut conclure que nous avons affaire au district de Terqa. Au temps de Samsi-Addu, les Hanéens possédaient des champs dans l'Ah-Purattim (I.6.22-43).

B. Bini-Yamina - Il semble que les Bini-Yamina qui habitaient l'Ah-Purattim, étaient désignés sous le nom de "Fils du fleuve" mārū nārim (A.1281). (*)

C. Bini-Sim'āl - Les Bini-Yamina du district de Saggarātum ont l'intention de razzier les villages des Bini-Sim'āl qui se trouvent dans l'Ah̆-Purattim (XIV. $84+.10-11,13,27-32,37-38,45-46$ ). Il pourrait s'agir du district de Mari. ${ }^{332}$

L'Ah-Purattim était peuplé de dizaines de villages où habitaient les tribus seminomades. L'information que nous avons tirée des textes de Mari peut être complétée par ce que $\mathrm{H}$. Charles raconte sur les 'Agêdât qu'il a visités en 1939.333 On peut supposer que le déploiement des villages des tribus au temps de Mari était semblable au déploiement des villages des 'Agêdât quatre mille ans après. Entre Abou Kémâl et Deïr Ez-Zôr il y a environ cinquante villages où habitent 10240 familles. Les habitants des villages sont divisés d'après leurs sous-tribus. Beau-

${ }^{331}$ Cfr. ci-dessus Chapitre II, § 3, L'année warki Tăb-şilli-Aššur.

332J.-M. Durand et D. Charpin, RA LXXX, p. 151.

${ }^{333}$ Voir la carte à la page 84 de son ouvrage Tribus moutonnières. 
coup de villages sont divisés en quartiers, et dans chaque quartier habite une hamūlā. Il y a des villages où on trouve seulement trois quartiers, et d'autres qui sont divisés jusqu'en quarante quartiers. Un village peut s'étendre sur $15-20 \mathrm{~km}$, et les villages sont limitrophes, de telle manière qu'on a l'impression que toute la vallée de l'Euphrate est une suite continue de villages. Chaque famille habite soit dans une maison en argile ( $d \bar{a} r)$, soit dans une maison dont les murs sont en argile et le toit en branches d'arbre (şibāt). La maison est construite sur la terre familiale (šerd). Quand la famille grandit, elle se ramifie en nouvelles maisons, et un nouveau quartier peut ainsi être créé, où habitera une ḩamūlā. Des deux côtés de la vallée de l'Euphrate s'étendent les pâturages d'hiver, qui sont assez près de la rivière - de une à cinq journées de marche. Dans la Chamieh (à l'ouest de l'Euphrate) on paît dans les lits d'oueds, où se trouvent des sources d'eau. Parfois on s'éloigne dans la steppe vers le nord et vers le nord-ouest, vers le Djebel Bichrî, qui est riche en puits et en pâturages. Dans la Djéziréh (à l'est de l'Euphrate) on paît dans la steppe qui est riche en sources. A l'époque de la transhumance les membres des tribus habitent dans les tentes. A cette époque ils ne sont pas nommés par les noms de leurs villages, mais par les noms de leurs sous-tribus. Pendant la période de la transhumance la conscience tribale des membres des tribus se renforce.

\section{Le district de Qattunăn}

A. Hanûm - Samsi-Addu écrit à Yasmahn-Addu à propos du recensement effectué par ce dernier du district de Qattunān et des Huanéens qui s'y trouvent (I.82. 12-15). Au temps de Zimri-Lim le gouverneur de Qattunān, Zakira-ḩammû, informe son roi que lorsque les Ḩanéens ont traversé le fleuve (Habur), un bateau appartenant à la localité de Țābātum a coulé (II.80.6-8). Enfin le commandant Ibalpì-El informe Zimri-Lim que "[tous] les Hanéens attendent [mon seigneur] à Zilhanan (qui se trouve dans le district de Qattunān)" (II.27.5-7), et Asqudum et Hali-hadun lui font savoir que "les Hanéens (qui sont venus de la steppe) se sont rassemblés à Qațtunān" (XXVI.31.15-16). 
B. Bini-Yamina - Une lettre nous raconte que les Bini-Yamina menacent le district et forcent les habitants des villages du district à s'enfermer dans les villes fortes (BENJ., p. 989b). Ces Bini-Yamina ne séjournent vraisemblablement pas dans le district même. Ce sont peut-être ces mêmes Bini-Yaminites à propos desquels il est dit, au moment où le district de Qattunān se réunit dans les places fortes: "il n'y a plus d'orge à disposition. Les Bini-Yaminites et la garnison qui est là viennent d'épuiser le grain" (XXVI.189.19'-21'). Enfin un document signale des Bini-Yamina qui sont venus de Qattunān (TH.84.18).

\section{Idamaras}

A. Hanûm - La présence des Hanéens dans l'Idamaraș, le triangle du Habur, était si marquée que les scribes ont mis parfois le déterminatif géographique $\mathrm{KI}$ après le nom des Hanéens en parlant des Hanéens de cette région, comme s'il s'agissait de leur pays (XVI/1, pp. 13-14). Maints documents nous informent sur leur présence dans cette région. ${ }^{334}$ Commençons par des allusions aux Hanéens sans préciser l'endroit spécifique où se déroule la scène: Du passage suivant nous apprenons leur présence dans l'Idamaraș: "Les Ḩanéens m’ont répondu: Nous n'irons pas sur l'Ah-Purattim. C'est au milieu de l'Idamaraș que nous habitons" (M.6148). Une lettre de Tell al Rimah nous apprend que les gens d'Idamaraş ont attaqué un nawûm des Hanéens (OBTR 9.12'-13'); rappelons enfin que KibriDagān est allé en Idamaraṣ pour amener de là-bas trois milles Ḩanéens (III.14; XIII.102; 103). (*)

a) L'Idamaraș supérieur: Išar-Lim, le commandant de l'armée de Yasmah̆-Addu, a effectué un recensement des Ḩanéens qui se trouvaient dans l'Idamaraș supérieur, dans les districts de Nahurur, Talhāyum, Kirdaḥat et Ašnakkum (V.51). Ce recensement est attesté aussi dans les documents de Chagar Bazar du 30.VI jusqu'au 15.IX de l'éponyme Adad-bani (1779/1778). Ibal-El, le merhum (le responsable du pâturage) d'Idamaraș, est allé à Ašlakkā pour conclure une alliance entre les rois d'Idamaraș et les Huanéens. A la suite de cette alliance les Hanéens se sont

${ }^{334}$ Cfr. J.-M. Durand, $R A$ LXXXII (1988), p. 113. 
rassasiés dans Ḩurrā et dans tout l'Idamaraş (II.37). Etant donné que la ville de Hurrā était près d'Ašnakkum (II.33.Rev.; AREP., p. 123a) et d'Ašlakkā (SIGN., p. 184b), on peut conclure qu'il s'agit des Hanéens de l'Idamaraş supérieur. Dans une lettre nous lisons à propos du lien qui existait entre les villes d'Ašnakkum et d'Ašlakkā d'une part et les Ḩanéens d'autre part: "Šadun-labā (le roi d'Ašnakkum) et Ibal-Addu (le roi d'Ašlakkā) sont arrivés ici auprès de moi, à Siharata avec les Huanéens ... Maintenant j'irai avec lui (Šadun-labā) et les Hูanéens dans l'intérieur du pays" (II.33.11'-13', 17'-18').

b) Apqum-ša-Habur: Pendant sa première année de règne, Zimri-Lim a distribué à Apqum-ša-Habur, la source du Habur, des anneaux aux femmes hanéennes (XXII.258).

c) Apum: Yanuh-Samar, peut-être le vizir de H̦āya-abum, le roi de Šehnnā/ŠubatEnlil, ${ }^{335}$ informe Zimri-Lim que les Hanéens sont installés près d'un lac du nom de Hुālabā $\bar{a}^{336}$ et entre les deux Saphum, celle du pays d'Apum et celle au sud du Djebel Sindjar ${ }^{337}$ (XXVI.358.3-6). $\left({ }^{*}\right)$

d) Kahat: Kabiya, le roi de la ville annonce à Zimri-Lim qu'un nawûm des Ḩanéens paît dans son district (II.59). (*)

e) Maḩānum: Les Hुanéens se sont rassemblés à Mahānum à l'occasion d'une pluie vue comme un signe néfaste (A.1191.4-6).

f) Naḩur: Un membre du clan Naḩan se trouve dans l'entourage d'Itūr-Asdu, le chef de la garnison mariote à Nahurur (A.2588.50).

g) Raşûm: Asqudum va de Suhum à Rașûm, entre le Habur et le Djebel Sindjar, ${ }^{338}$ pour recruter des Ḩanéens qui se trouvent dans la steppe (XXVI.40.1929).

h) Sinamum: Les Hanéens ont dû recevoir une grande quantité de laine et d'orge du roi de Sinamum (B.590.4-14). ${ }^{339}$

${ }^{335} \mathrm{XXVI} / 2$, p. 130.

${ }^{336}$ D'après D. Charpin c'est la région inondée du oued ar-Rad (XXVI/2, p. 135, n. b).

${ }^{337} \mathrm{XXVI} / 2$, p. 135 , n. c.

${ }^{338} \mathrm{XXVI} / 1$, p. 178, n. d.

339J.-R. Kupper (Nomades, p. 15) pense qu'il s'agit de marchands hanéens, J.T. Luke (Pastoralism , p. 162) quant à lui suppose que les H̆anéens en occurrence sont des soldats. 
i) Subat-Enlil: Les Hanéens ont razzié les troupeaux du palais de Subat-Enlil et des notables (IV.80.3-5).

j) Šnā, Urgiš et Šnah̆: Les Anciens de Sunā, Urgiš et Šenahn et un Ḩanéen du nom de Yataphu apparaissent dans une liste qui enregistre des envois de bovins et ovins à un employé de Zimri-Lim (XXIII.504). Il se peut qu'une lettre d'IšmeDagān à Yasmahy-Addu, IV.40, nous renseigne sur les relations entre les Huanéens et les villes d'Urgiš et Šnah (cfr. XXIII, p. 434).

k) Sunhum: Subram, le gouverneur de Subat-Enlil,340 écrit à Zimri-Lim au sujet d'un litige qui oppose les habitants de la ville de Šnā et les habitants d'Apum à propos de l'appartenance de la ville de Sunhum soit au clan Yabasu soit à la tribu Ḩanûm (A.1251).

1) Tādum: Un messager informe Yaqqim-Addu, le gouverneur de Saggarātum, que "Ḥāya-Sūmu (le roi d'Ilānșurā) assiège (la ville de) Hazzakanum, et les Ḩanéens portent le grain de Tādum" (XIV.120.13-16).

m) Talhāyum: Des Ḩanéens se trouvaient sous l'ordre de Ḩammi-epuḩ, le roi de la ville (A.1610+.40-43).

n) Zalluhan: D'après le témoignage du roi de Zalluhan, Zakura-abu, les Hुanéens faisaient partie des habitants de son district (B.81.36-38).

o) Zurrā: 253 Ḩanéens ont été recensés dans la ville en l'année ZL 11' (M.5705. VI.1-3).

B. Bini-Yamina - Il semble qu'on n'ait pas de preuve de la présence constante de Bini-Yamina dans cette région. ${ }^{341}$ G. Dossin cite un passage où il est dit que "les Bini-Yamina se sont rassemblés dans Yaḩmumum" (BENJ., p. 986c)..$^{342}$ Cette ville était située près d'Ilānşurā (A.49.59-62), donc en Idamaraş. Mais on ne sait pas s'il s'agit d'un séjour permanent dans la région ou d'un séjour temporaire. Il faut aussi signaler le fait que les Bini-Yamina sont mentionnés en relation avec

340 D. Charpin, M.A.R.I. V, pp. 134-135.

341 Voir H. Klengel, Benjaminiten und Hanäer, p. 213 et voir la carte chez H. Klengel, Zwischen Zelt und Palast, p. 51.

${ }^{342}$ Monsieur M. Birot m'a écrit le 5 septembre 1982 que c'est le seul témoignage de la présence de Bini-Yamina dans l'Idamaraş. 
Adūna-Addu d'Idamaraş. D'après un témoignage, on voit que Adūna-Addu refuse d'aboutir à un accord avec les Bini-Yamina (BENJ., p. 990a) et d'après un autre témoignage, que les Uprapûm se préparent à conquérir son pays (S. 115, no. $72-17,11.5-8$ ). Un autre témoignage de la présence des Bini-Yamina en Idamaraş se trouve peut-être dans le fait que Qarni-Lim, le roi d'Andarig, a conclu une alliance avec eux quand il combattait à côté d'Ešnunna dans l'Idamaraş (BENJ., p. 990b; AREP., p. 108 + BENJ., p. 991a). ${ }^{343}$ Enfin, Ḩammi-ištamar, le roi d'Uprapûm, dément l'information que lui a transmise plusieurs fois le merhum, Ibal-El, d'après laquelle les Uprapéens ne cessaient de voler les Bini-Sim'āl (A.2094).

C. Bini-Sim'āl - Ibal-El qui se trouve en Idamaraş rassure son roi, Zimri-Lim, que "le campement pastoral (nawûm) et les Bini-Sim'āl vont bien" (II.33.21'-22'; 37.25). ${ }^{344}$ Un intéressant témoignage sur les Bini-Sim'āl en Idamaraş se trouve dans une lettre de Yawi-Ilā, le roi de Talhāyum, qui informe Zimri-Lim: "De la même manière qu'auparavant, du temps de Yahdun-Lim, ton père, nous avons remis aux Bini-Sim'āl le pays de Yapturum, la ville de Talhāyum et ses villages" (XIII.144.26-29). D'après D. Charpin et J.-M. Durand ${ }^{345}$ l'appellation "BiniSim'āl" se réfère ici à Zimri-Lim ou à Yahdun-Lim. Quant à nous, nous préférons y voir la tribu elle même. Peut-être faut-il supposer comme M.B. Rowton ${ }^{346}$ que la ville qui appartenait à l'origine aux Bini-Sim'āl, fut prise par Zimri-Lim, et que maintenant elle leur est rendue. Une autre lettre nous apprend que les rois "frères" de Sibkuna-Addu, le roi de Sudā, lui reprochent d'avoir conclu une alliance avec Zimri-Lim et les Bini-Sim'āl (BENJ., p. 991b). Il s'agit sans doute des BiniSim'āl qui séjournent près de Sudā. Enfin, les Bini-Sim'āl vont conclure une autre alliance avec les Anciens de la ville de Hurrā (A.641).

343J.-R. Kupper, Nomades , p. 48 ajoute BENJ., p. 992a.

${ }^{344}$ Cfr. aussi A.1610+.19-20; A.1212.18-19: "les Bini-Sim'al et les armées vont bien".

D. Charpin et J.-M. Durand ( $R A$ LXXX, p. 152 et n. 57) signalent l'existence de nombreux inédits où on trouve aussi cette phrase.

$345_{\text {RA LXXX, p. } 152 .}$

346 JNES XXXII (1973), pp. 212-213. 
D. Yamutbal: A propos des gens de Yamutbal en Idamaraş, nous apprenons par un passage d'une lettre qu'Iși-Nabû le Yamutbaléen commande trente Yamutbaléens, Huabirū (A.2939.11-14). Il s'agit sans doute des membres de la tribu Yamutbal qui ont quitté leur tribu pour devenir des Habirū, c'est à dire des déportés. Il faut signaler en outre que tous les Huabirū qui apparaissent dans les documents de Mari se trouvent en Idamaraş.

E. Yusan: Une ville, située à l'est de l'Idamaraş s'appelait Razamā-Yusan. ${ }^{347}$ On peut supposer que près de cette Razamā vivaient des membres du clan Yusan inconnu par ailleurs.

\section{La région d'amont}

A. Ḩanûm - a) Ahunā: Yaqqim-Addu, le gouverneur de Saggarātum, informe Zimri-Lim que les Hanéens ont quitté la ville d'Ahunā pour aller vers les pâturages (XIV.92.19-20).

b) Subat-Samaš: Samsi-Addu fait savoir à son allié Išhhi-Addu, le roi de Qatanum, qu'il a écrit à Sîn-tiri, le gouverneur de Subat-Samaš, afin que celui-ci rassemble tous les Hanéens pour razzier les moutons de Sümu-epuh, le roi d'Alep, et des Rabbûm (I.24+.39-42). Nous avons déjà vu (ci-dessus Chapitre III, Introduction) que l'on a demandé à Sîn-tiri des informations concernant les tribus Bini-Yamina et Bini-Sim'āl qui forment ensemble la tribu Hanûm (A.2560.4-8). (*)

B. Bini-Yamina - De même que les Hanéens étaient liés tout particulièrement à l'Idamaraş, les Bini-Yamina étaient liés à la région d'amont, la région située entre le Balīh et le Haut Euphrate, ${ }^{348}$ et c'est la raison pour laquelle les scribes mettaient

347D. Charpin et J.-M. Durand, RA LXXXI, p. 129, n. 8.

348 A cette région se réfere l'adverbe elênum qui est à traduire "en haut, en amont" cfr. $C A D \mathrm{E}$, p. $85 \mathrm{~b}(2) ; A H w$, p. $198 \mathrm{~b}(B .2)$ et la traduction de M. Birot de XIV.89.7, et H. Klengel $Z_{w i-}$ schen Zelt und Palast, p. 58. Monter là-bas est exprimé par l'adverbe eliš et descendre de làbas par l'adverbe ištu elênum. Nous n'identifions pas ces adverbes avec "le haut pays" mătum elitum, qui se réfère, à notre avis, au Zalmaqum. 
parfois le déterminatif géographique $\mathrm{KI}$ après les noms des Bini-Yamina en parlant des Bini-Yamina de cette région. ${ }^{349}$ Là se trouvaient des villes-royaumes des Bini-Yamina où régnaient leurs rois du temps de Yahdun-Lim (Tuttul et Abattum) et aussi sans doute du temps de Zimri-Lim; dans cette région transhumaient aussi les membres de la tribu qui habitaient l'Ah̆-Purattim. Yahdun-Lim mena une série de campagnes dans cette région; il conquit Tuttul, Abattum, qui se trouvent sur l'Euphrate entre le Balịh et le coude de l'Euphrate, et Imār, Zalpaḥ sur le Balīh et Pahudar à l'est de Harrān. Le but de ces conquêtes était, nous semble-t-il, de s'emparer du pays des Bini-Yamina à la suite de sa victoire sur eux à Samānum dans le district de Terqa.

Plusieurs villes qui sont liées aux Bini-Yamina sont connues par leurs noms:

a) Abattum: Au temps de Yahdun-Lim régnait dans la ville Ayālum, le roi de Rabbûm (M.2802.III.8-9). D'après un nom d'année de Yahdun-Lim il a remporté une victoire sur les Bini-Yamina et Imär à la porte d'Abattum (T.322+). Au temps de Zimri-Lim, nous lisons dans une lettre de Yaqqim-Addu, le gouverneur de Saggarātum, que les Bini-Yamina ont l'intention de conquérir Abattum (XIV.84+.14'-15'); dans une autre lettre, il informe Zimri-Lim que Dādi-hadun, le roi des Rabbûm, "moissonne son orge à Abattum" (XIV.83.36-37), d'où on peut déduire que Dādi-hadun contrôlait Abattum. En effet, c'est entre Tuttul et Imār que Dādi-hadun a l'habitude de traverser l'Euphrate lors de ses transhumances (A.9). La traversée était peut-être près d'Abattum où justement Samsi-Addu conseille à un chef nomade du nom de Zimrān de faire la traversée $(I .91+.17$ '18'). Le fait que Dādi-hadun habite dans la région explique pourquoi il est apte à servir d'intermédiaire à Zimri-Lim qui désire acheter Alaḥtum. ${ }^{350}$ Cela explique aussi les cadeaux qu'il a envoyés à Zimri-Lim lorsque celui-ci s'est trouvé à Yakaltum et à Halab allant vers Ugarit (XXV.38+; 48+; XXIII. 542.36), et le cadeau envoyé à son vizir Sumat-Erah lorsque Zimri-Lim s'est trouvé à DūrSūmu-epuh (XXV.136.1-5). (*)

b) Ahūna et Zalpah̆: Il est fort probable que ces deux villes appartenaient aux BiniYamina, ou au moins étaient incluses dans leur zone d'influence: "Les sugāgū des

${ }^{349}$ XVI/1, p. 39; J.T. Luke, Pastoralism, pp. 55, $62,66$.

350 D. Charpin et J.-M. Durand, RA LXXX, p. 148 . 
Bini-Yamina se sont rassemblés à Zalpaḩ, et puis ils sont allés à Ahūna, et se sont assis < devant> Șū[ra-hammû (le roi d'Amnanûm) et Yarìm-Lim (le roi de Yahrurûm)]" (II.53.12-16). ${ }^{351}$ Ils exigent de leurs rois d'aller chez Zimri-Lim pour réclamer leurs villes (dans l'Ah-Purattim, sans doute). Ils insistent en outre pour qu'un certain Lahuun-Dagān parte aussi (11. 20-25). Ce Lahuun-Dagān était un uprapéen (cfr. XVI/1, p. 142). Peut-être faut-il l'identifier avec un madārum (roi vassal) uprapéen de la ville de Subat-Samaš (XIV.88.14-16). Les rois du Zalmaqum qui sont entrés à Zalpah, invitent les rois des Bini-Yamina à un palabre (A.2526). Sur le lien existant entre les Bini-Yamina et la ville d'Ahunā on peut tirer parti aussi des faits suivants: 1 . Un messager de Șūra-hammû est parvenu à Mari depuis Ahunā (M.8990). 2. Dans les listes de messagers qui arrivent à Mari, les Bini-Yamina sont inscrits à côté des gens d'Ahunā. 352 3. Un voyageur qui allait à Ahunā a rencontré sur son chemin "un campement pastoral (nawûm) des Bini-Yamina" (BENJ., pp. 986d-987a). ${ }^{353}$ 4. Dans la ville d'Ahunā sont entrés les trois rois des Bini-Yamina: Hardûm, Samsi-Addu et Yagih̆-Addu (XXVI.24.6-7). Enfin il faut signaler que Zimri-Lim a ordonné de détruire les parcs à moutons et les troupeaux des Hanéens-Bini-Yamina et de leur faire passer le Balīh (A.1086), vraisemblablement vers la rive ouest, dans la région des BiniYamina.

c) Halabit (la moderne Halébiyé354): Dans une lettre envoyée depuis Aḥunā et Ṣerdā nous lisons: "Les Yahruréens, après avoir passé quelques jours dans la forêt, ont traversé (l'Euphrate) à Halabit" (A.1176).

d) Subat-Samaš: Yasmahn-Addu demande à Sîn-tiri, le gouverneur de SubatSamaš: "les Hanéens qui ont traversé, Bini-Sim'āl (ou) Bini-Yamina, quelle est leur tribu ?" (A.2560.6-8).

e) Tuttul: Au temps de Yaḩdun-Lim régnait dans la ville Baḩlu-kulim, le roi d'Amnanûm (M.2802.III.6-7). Nous avons des témoignages sur le lien qui existait du temps de Zimri-Lim entre la ville et les tribus des Bini-Yamina. Lana-

${ }^{351}$ Cfr. M. Anbar, Biblica XLIX (1968), pp. 227-229.

352VII.159.12-15; 165.10-12; 210.13'-18'; 211.15-19.

${ }^{353}$ Cfr. XXVI.24.32.

${ }^{354} \mathrm{XXVI} / 1$, p. 126. 
sûm, le préfet de Tuttul, raconte dans deux lettres que les Uprapéens sont allés à Tuttul (A.2769.5-7; A.4530-bis.5'-6'). Dans une autre lettre, on parle de Yarihéens qui vont entrer à Tuttul (XIV.55.15-17), et Ibal-El le merhum (préposé aux pâturages royaux), écrit à Zimri-Lim que "Șibān lui a apporté des renseignements concernant les rois des Bini-Yamina et des renseignements concernant Lanasûm" (II.36.10-14). A une intrigue visant à expulser Lanasûm de son poste à Tuttul a pris part Yasmah-Addu, le roi des Yarihûum (II.137). Dans cette lettre, nous lisons que Lanasûm "circule parmi tous les Bini-Yamina et dans les environs de la ville de Tuttul" (II.137.27-29) pour percevoir la taxe qu'ils doivent au Palais. Le même Lanasûm annonce à Zimri-Lim que les Bini-Yamina et les rois du Zalmaqum ont l'intention de conquérir la ville de Tuttul (A.215). Les Bini-Yamina ont eu l'habitude de faire des razzias dans les environs de Tuttul. Contre cette menace l'employé Hali-hadun conseille à Zimri-Lim d'envoyer un renfort à Lanasûm (BENJ., p. 987d). Tous ces témoignages montrent que les Bini-Yamina du temps de Zimri-Lim n'habitaient pas à Tuttul, mais dans ses environs. (*)

C. Bini-Sim'āl - a) Subat-Š̀amaš: Nous avons déjà cité plusieurs fois la lettre où Yasmah-Addu demande à Sîn-tiri, le gouverneur de la ville, si une tribu qui a traversé l'Euphrate était formée de Bini-Yamina ou de Bini-Sim'āl (A.2560.4-8).*

\section{Zalmaqum}

A. Bini-Yamina - Asditakum, le roi de la ville de Harrān, et les autres rois du Zalmaqum ont conclu une alliance avec les Bini-Yamina dans le sanctuaire de Sîn à Harrān (XXVI.24.10-12). Dans la contrée du Zalmaqum se trouve un territoire des Bini-Yamina (BENJ., p. 991b). En outre nous avons des informations concernant des activités des Bini-Yamina en commun avec les rois du Zalmaqum (BENJ., pp. 986b; A.215).

\section{Mont Bisir}

A. Bini-Yamina - Les Bini-Yamina se déplaçaient vers le Mont Bisir à l'époque de la transhumance (V.27.25-26). Il peut s'agir des Bini-Yamina qui habitent la région du Balīh̆, mais peut-être s'agit-il des habitants d'Ah-Purattim, 
car H. Charles raconte que la fraction des 'Agêdât, les Šwêt, qui habitaient cinq villages au sud d'El Aachâra, l'ancienne ville de Terqa, transhumaient au Djebel Bichrî (= Mont Bisir). ${ }^{355}$ Une lettre de Samsi-Addu à Yasmah-Addu (I.85+) nous apprend qu'une armée de 20000 hommes va partir pour Qatanum. Elle doit traverser le désert par l'un de ces trois chemins: celui qui part d'Abattum, celui qui part de Halabit ou celui qui part de Mari elle-même. Le choix dépend de l'existence de points d'eau. Ce sont les membres du clan Uprapûm qui connaissent ces chemins qui vont examiner la question de l'eau (1l. 21-25). On peut conclure du fait qu'on s'adresse aux Uprapûm qu'ils ont l'habitude de parcourir le désert entre Mari et Qatanum.356

B. Sutûm - Haammi-talû, le chef Sutéen, confirme à Bahdi-Lim, le préfet du palais de Mari, que les Sutûm qui circulaient dans le Mont Bisir ont trouvé une bourse d'argent d'une très grande valeur (VI.44). Comme les bédouins des temps modernes, ${ }^{357}$ les Sutûm ont fait des razzias dans les oasis de Tadmer et de Našalã (V.23).

Les Sutûm sont chez eux dans le désert qui sépare Mari de Qatanum. Pour cette raison Samsi-Addu demande à Yasmah̆-Addu de s'adresser à Ḩammi-talû pour qu'il accompagne une caravane qui va traverser le désert (I.17.37-43).358

\section{Yamb̧ad, Qatanum et Amurrum}

A. Bini-Yamina - D'après une information transmise par G. Dossin, dans les archives on trouve des documents qui attestent la présence des Bini-Yamina à Yamhad, Qatanum et Amurrum (RHPR XXX [1950], p. 7). Le clan de Rabbûm habitant la région d'amont avait l'habitude d'émigrer au pays de Yamhad (I.6.912; IV.6.5-12). Les Uprapûm voulaient se rendre chez les Rabbûm, probablement

355 Tribus moutonnières, p. 118.

${ }^{356}$ Cfr. J.-M. Durand, M.A.R.I. V, pp. 162-163. (*)

${ }^{357}$ E. Wirth, Syrien, Darmstadt 1971, pp. 444-446.

358 J.T. Luke, Pastoralism, p.128. 
au Yamb̧ad (BENJ., p. 985a.12'-14'). ${ }^{359}$ Sîn-tiri, le gouverneur de Subat-Šamaš, va rassembler tous les Hanéens pour razzier les moutons de Sümu-epuh, le roi d'Alep, et des Rabbûm (I.24+.39-42). Il s'agit ici aussi des Rabbûm qui se trouvent au Yamhad. Șūra-ḩammû, le roi d'Amnanûm, a proposé à Dādi-ḩadun, le roi de Rabbûm, de razzier les parcs à moutons des Yahrurûm et de faire passer leurs troupeaux au-delà de l'Euphrate (A.3821.19-21). Hammi-ištamar se rendait à Ugarit pour conclure une alliance avec Zimri-Lim dans le temple d'Addu de Halab (A.2094.9-10). Le fait que Zimri-Lim ait racheté à Ugarit des Hanéens, probablement appartenant à son armée, aux Bini-Yamina montre que ceux-ci étaient présents dans cette contrée (XXIII.540; M.11351). J.-M. Durand nous informe en outre d'après des textes inédits qu' "on retrouve après la victoire de Zimri-Lim sur Yasmahn-Addu, des chefs yaminites réfugiés à Qatana". ${ }^{360}$ Nous avons déjà cité plusieurs fois la lettre A.2560 où Yasmah-Addu questionne Sîntiri à propos des Bini-Yamina ou des Bini-Sim'āl qui ont traversé l'Euphrate en direction de la Djéziréh (ana ebirtim, 1. 4). On peut supposer qu'il sont venus du Yamḩad.

B. Bini-Sim'āl - Voir la lettre A. 2560 ci-dessus.

\section{La région du Tigre}

A. Bini-Yamina - a) Ekallātum: Des membres du clan Yaḩrurûm qui habitaient dans les alentours d'Ekallātum (à une journée de marche au nord d'Aššur) furent recensés par Išme-Dagān lors du recensement d'Ekallātum (II.18.4-6). ${ }^{361}$ Par contre Aškur-Addu, le roi de Karanā, informe que "deux mille Turukkéens, deux mille Qabréens et un millier de Yahruréens ont razzié la main-d'oeuvre qui s'affaire au canal d'Išme-Dagān. Išme-Dagān est allé à (son) secours mais ils ont remporté la victoire sur lui" (XXVI.510.8-15). On peut conclure de ce passage que le Yaḩrurûm stationnait à l'est du Tigre et au nord du Zāb inférieur. J.-R.

${ }^{359}$ Voir J.-R. Kupper, Nomades, p. 51.

360 M.A.R.I. V, p. 163.

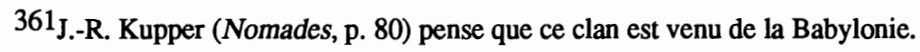


Kupper ${ }^{362}$ fait appel dans le présent contexte à un document de la Babylonie où on parle de trois hommes originaires de Idiqlat-Yahrurûm "le Tigre de Yahrurûm" ( $V A B$ VI.141.7-8).

B. Numḩāa - a) Ekallātum: Yamşium, le représentant de Zimri-Lim à Ilānșurā, informe Zimri-Lim que "Tous les Numhéens d'Ekallātum ne cessent d'errer faute de grain, et ils transportent le grain de Karanā" (XXVI.342.5-8).

C. Ya'ilānum - a) Qabrā: Cinq jours après avoir vaincu la ville de Qabrā, Samsi-Addu a aussi infligé une défaite à la tribu Ya'ilānum (I.92.5-10). La bataille elle-même a eu lieu dans la ville de Tutarwi (IV.33).

\section{Le sud du Djebel Sindjar}

A. Hanûm - a) Andarig: Qarni-Lim, le roi d'Andarig, est venu avec les Hanéens pour rejoindre les rois d'Idamaraș (A.1610+.11; A.1212.11), et Atamrum, qui est devenu le roi d'Andarig après Qarni-Lim, ordonne au vizir de Zimri-Lim, Habdu-Malik, d'aller avec le merhûm (Ibal-El ?) rassembler les Hanéens et de venir avec eux à son secours (XXVI.389.11-15; 394.10'-12').

b) Haamadānum: Les Ešnunnéens qui sont montés dans la région interrogent les Hanéens qui se trouvent à Hamadānum sur la sécurité du passage au pacage (XXVI.508).

c) Kasapā: Dans une lettre de Hali-ḩadun nous lisons: "Les Hanéens et Sarrāya (le roi de Razamā) sont rassemblés à Kasapā pour t'(Ešnunna) affronter" (A.988.1516).

d) Kurdā: Dans un mémorandum qui concerne des razzias nous lisons entre autres: "A propos de quatre Hanéens qui ont été razziés à Kurdā avec leurs moutons et qu'on a fait entrer dans l'ergastule" (XXIII.592.1-4).

e) Razamā: Šarrāya, le roi de Razamā, va avec les Ḥanéens à Kasapā pour faire face à l'ennemi (A.988.15-16).

${ }^{362}$ Nomades, p. 53. 
f) Saphum: Les Hanéens se sont rassemblés à Saphum, au nord ${ }^{363}$ ou au sud s.64 $^{364}$ du Djebel Sindjar, avant de partir avec Asqudum pour une expédition dans l'Idamaraș (XXVI.30.8-9).

B. Numhāa et Yamutbal - a) Andarig: La ville d'Andarig était située dans un pays qui portait le nom de la tribu Yamutbal: "le pays de Yamutbal" (X.84.24-25; XXVI.432.8'). Ainsi Yasīm-El rassure Zimri-Lim que "La ville d'Andarig, l'armée de mon Seigneur et le pays de Yamutbalum vont bien" (XXVI.432.7'-9'). Quand Hammurabi, le roi de Kurdā, déclare: "Yamutbalum est mon ennemi" (XXVI.522.22'-23'), il se réfère sans doute au pays d'Andarig. Nous apprenons aussi par une lettre de l'employé Hali-hadun à Zimri-Lim qu'un conflit a éclaté entre la ville d'Andarig et les tribus Numhā et Yamutbal: "Mon seigneur réside à Ašlakkā et il m'a envoyé au district de Numhāa et de Yamutbal. [J'ai] ainsi [établi] un pacte d'amitié entre [ ... ] Qarni-Lim (le roi d'Andarig), [Numhāa] et Yamutbal" (X.157.8-16). En outre, Qarni-Lim s'occupe d'un homme de Yamutbal dont les troupeaux ont été razziés (II.79.10-23).

b) Hamadānum: On parle de Huamadānum de Yamutbal (A.1097), mais la ville était considérée comme la ville de Yanșib-Addu (qui fait partie) du Numhāa (XXVI.419.6-7).

c) Harbû: Ibal-pī-El informe Zimri-Lim que "les armées (des rois de l'Idamaraș et de Qarni-Lim) ont marché vers Harbû de Yamutbal" (A.1610+.14-15).

d) Karanā: Karanā est la capitale du "pays de Numḩā" (XXVI.412.12; $521.11), 365$ où se trouve, sans doute la ville "Ašān des Numhéens" (XXVI.415.7). C'est à propos de ces Numḩéens, sans doute, que Huammurabi, le roi de Kurdā, écrit à Išme-Dagān: "Nous n'avons qu'un seul ennemi; ne t'en prends donc pas aux Numḩéens" (XXVI.511.17-19). Atamrum, le roi d'Andarig, quant à lui est d'accord d'envoyer en renfort à Karanā 600 Yamutbaléens, si Aškur-Addu lui envoie à leur place des Numḩéens sujets du roi de Karanā

\footnotetext{
${ }^{363}$ Voir XXVI/1, p. 163, n. a

${ }^{364} \mathrm{XXVI} / 2$, p. 135 , n. c.

${ }^{365}$ Voir aussi XXVI.358.9'.
} 
(XXVI.394.20'-23'). ${ }^{366}$ De même Atamrum, le roi d'Andarig, propose à AškurAddu, le roi de Karanā, de le rencontrer dans la ville de Șidqum qui se trouve: "à la frontière du Numhāa de Karanā et du Yamutbalum supérieur" (XXVI.404.7).

e) Kurdā: Tout d'abord nous savons que Simahlānē le roi de Kurdā était originaire de la tribu Numhā (X.5.4). ${ }^{367}$ En outre, nous sommes renseignés sur le lien qui existait entre la ville de Kurdā et la tribu Numhãa du fait que Bunu-Ištar, le successeur de Simahlānē, a envoyé un personnage nommé Silillum avec l'armée des Numhāà à Kasapā contre Qarni-Lim, le roi d'Andarig (II.130.11-13). La même lettre nous apprend aussi que, sur l'ordre de Bunu-Ištar, les rois d'Idamaraș ont garrotté des membres de la tribu Yamutbal qui se trouvaient dans le palais de Subat-Enlil (11. 14-18). C'est peut-être comme Numhéens au service de Hammurabi, le roi de Kurdā, qu'ils interviennent dans le rachat d'un homme de Terqa (XXVI.421.15'-21'). Un prisonnier numhéen, qui faisait probablement partie de l'armée de Kurdā 368 est sujet à discussion à propos de son éventuel rachat, qui ne se réalisa pas à cause de l'assassinat de cet homme (XXVI.434.5). Sept autres prisonniers Numhéens, appelés "esclaves", sont l'objet d'une dispute évoquée dans la lettre de Yamșûm, l'envoyé de Zimri-Lim à Ilānșurā (XXVI.302.16-32). Enfin, on connaît une localité qui porte le nom de Rașûm-ša(= de)-Yamutbal (XXIII.569.6-7; 570.6-7).

f) Razamā: Išme-Dagān a recensé le pays de Razamā-Yamutbalim (II.18.7-8), c'est à dire Razamã qui se trouvait dans le pays de Yamutbal, à l'instar de Maškan-šapir, qui était située dans le pays de Yamutbal. ${ }^{369}$ La ville de Razamā est à localiser au sud-est du Djebel Sindjar. ${ }^{370}$ Par l'appellation Razamā-Yamutbalim, nous devons comprendre qu'il s'agit d'une contrée près de Razamā où vivaient des membres du clan Yamutbal. A cette contrée se réfère probablement la lettre A.505 qui "indique qu'en dépit de la conduite de Hatnu-rabi [le roi de Qațarā ou

${ }^{366}$ Cfr. XXVI/2, p. 228 , n. h.

${ }^{367} \mathrm{XXVI} / 2$, p. 338 , n. b: "La région occupée par les Numhéens s'étendait sur les royaumes de Karanâ et de Kurdâ".

${ }^{368} \mathrm{XXVI} / 2$, p. 338 , n. b.

${ }^{369} \mathrm{XXVI} / 2$, p. 148.

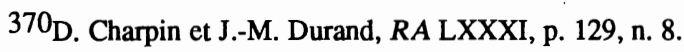


de Karanā], qui a précédemment pillé le Yamutbal ... les gens du Yamutbal n'ont pas lancé d'attaque contre le pays de Karanā".371

\section{Subum}

A. Hanûm - Abi-mēkim écrit à Zimri-Lim un rapport sur les négociations qu'il mena avec Hammurabi, le roi de Babylone, à propos d'une alliance que Zimri-Lim et Hammurabi vont conclure. Hammurabi refuse d'inclure dans l'accord trois villes frontières: la ville de Yumahammû, Id et Yabliya (XXVI.468.15). Par la suite, dans un passage endommagé, Abi-mēkim fait mention de "trois mille Hanéens Yumaḩammû" (1. 22). On voit donc une ville appelée d'après le clan qui y habite. Dans une longue liste de sugāgū de la tribu Hanûm nous trouvons un personnage du nom de Kibsi-Addu (XXIV.23.III'.14'). Or, d'après VII. 213.10 il remplissait sa fonction à Suhum. Nous avons donc ici un témoignage de la présence des Hanéens à Suhum. Dans une lettre d'Asqudum sont mentionnées les paroles que Yarìm-Addu de Hurbān adresse au pays: "Les Hanéens sont venus pour votre nourriture" (XXVI.36.23-24). Il ne s'agit pas ici, nous semble-t-il, des habitants de la région, mais bien de l'armée hanéenne qui cherche à se rassasier. Ce sont les même Hanéens conduits par Ašmad, qui réclament leur part des prisonniers Rapiquéens (XXVI.37.12'-13').

B. Bini-Yamina - Meptûm, le gouverneur de Hanat, a envoyé à Abi-mēkim un homme de Hurbān et un homme du clan Amnanûm, qui rapportent les paroles de Meptûm à Ešnunna (XXVI.456.13-18). Ce fait ne suffit pas, bien entendu, à conclure que les Bini-Yamina séjournaient à Suhum.

C. Sutûm - a) Yabliya: Yasmah̆-Addu a écrit à Samsi-Addu que mille Sutéens avaient l'intention de razzier Yabliya (dans la région de Hït - Hadīta; I.100.6-8). Un mémorandum signale une razzia de Sutûm sur Harbē et Ayabē qui sont près de Yabliya (XXIII.592.4-6). (*)

${ }^{371}$ D. Charpin et J.-M. Durand, RA LXXXI (1987), p. 135, n. 31. 
b) Rapiqum: Il est très possible que ce soient toujours les Sutûm qui ont fait une razzia sur Rapiqum (près de Fallūjah; I.83.15-21). 372

Dans le présent contexte il faut mentionner une lettre où il est dit que dans un rêve de Zimri-Lim les Sutéens lui déclarent: "Tant que tu ne rendras pas nos demeures, nous ne les libérerons pas" (XXVI.225.10-12). On ne dit pas où ces Sutéens se trouvent, mais il pourrait très bien s'agir du Suhum. ${ }^{373}$ On ne peut pas savoir à quoi se réfère le mot " nos demeures" qui est écrit ta-šu-ba-at-ni ou šu-ba-at-ni. ${ }^{374}$ S'agit-il de villages ou de campements?

\section{Résumé}

Le résumé de la discussion sera organisé tribu par tribu.

A. Hanûm - Les Hanéens possédaient des localités dans les districts de Mari et de Saggarātum et peut-être à Suhum. De leurs localités du district de Mari, ils transhumaient vers les pâturages, soit dans la Djéziréh, soit dans le Chamieh ou dans les deux. Des troupeaux hanéens paissaient dans le district de Terqa. Nous trouvons les Hanéens dans le district de Qattunān et dans la région d'amont, mais nous ne savons pas s'ils avaient là-bas des villes ou s'ils transhumaient seulement dans ces régions. Les Hanéens ont eu un lien spécial avec l'Idamaraş. Nous ne savons pas s'ils avaient des villes dans cette région. A l'époque de Yaḩdun-Lim ils avaient une ville-royaume dans la vallée de l'Euphrate.

B. Bini-Yamina - Les Bini-Yamina possédaient des localités dans les districts de Mari, de Terqa et de Saggarātum. De ces localités ils transhumaient vers la région d'amont. On trouve des Bini-Yamina dans le Zalmaqum, dans la région du Tigre, dans les pays de Yamhad, Qatanum et Amurrum et dans les alentours d'Ekallātum et de Qabrā et peut-être aussi parfois en Idamaraș. Une de leurs régions de pâturage était le Mont Bisir. Au temps de Yahdun-Lim existaient trois

372 J.-R. Kupper, Nomades , p. 90, n. 2.

${ }^{373} \mathrm{XXVI} / 1$, p. $466 \mathrm{a}$.

374J.-M. Durand (ibidem) écarte la seconde possibilité, car "le texte porte un très clair TA". Pourtant, vu l'alternance possible entre TA et SA dans l'écriture de Mari (XV, p.27, no. 202, n. 1) on doit, nous semble-t-il, la laisser en considération. 
villes-royaumes des Bini-Yamina dans la vallée de l'Euphrate; au temps de ZimriLim ils possédaient des villes-royaumes dans la région d'amont.

C. Bini-Sim'āl - On trouve les Bini-Sim'āl en Idamaraș dans la région d'amont et peut-être dans le district de Mari.

D. Sutûm - On trouve les Sutûm dans le Mont Bisir, dans le désert entre Mari et Qatanum et dans les districts de Terqa et de Suhum.

E. Numba et Yamutbal - On les trouve dans les districts de Terqa, de Saggarātum et au sud du Djebel Sindjar.

F. Ya'ilānum - On trouve cette tribu à l'est du Tigre au nord du Zāb inférieur. 


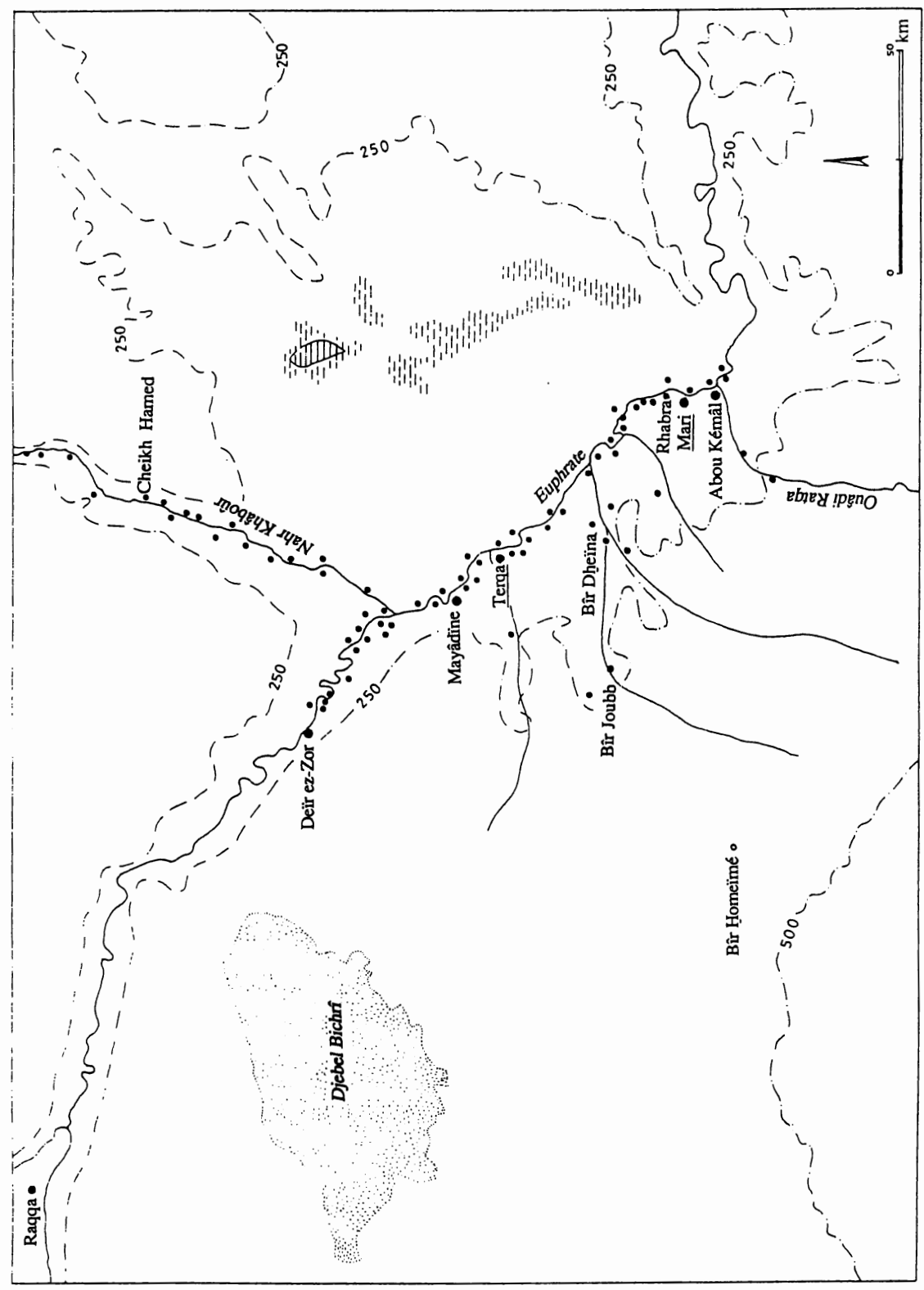

2. Le Moyen-Euphrate entre Abu Kémâl et Deïr ez-Zor 


\section{Chapitre V: Les dirigeants tribaux}

Dans ce chapitre nous allons examiner ce que nous apprenons des textes de Mari à propos des dirigeants tribaux, quels étaient leurs titres et ce que signifient ces titres.

\section{Les rois}

A. Les rois des Hanéens - La grande inscription de fondation de YahdunLim (M.2802) relate l'expédition de Yahdun-Lim jusqu'à la Méditerranée et ses guerres contre les Bini-Yamina et les Hanéens. La victoire sur les Hanéens est décrite par ces mots: "Il détruisit la ville de Haman, le centre des Hanéens, que tous les pères des Hanéens avaient construite et il la réduisit en tells et en terres arables. En outre, son roi, Kașūri-Hala, il le fit prisonnier, il a pris leur pays et ainsi il domina complètement les rives de l'Euphrate" (III.28-IV.4). La courte inscription de Yahdun-Lim (IAHD.) raconte elle aussi la victoire sur les Hanéens: "Dagān proclama ma royauté. Il me donna l'arme puissante qui terrasse les rois mes ennemis, et (ainsi) sept rois, les pères des Hanéens, qui avaient combattu contre moi, je les fis prisonniers. Je reconquis leur pays, j'ai dominé401 les rives de l'Euphrate et je fis demeurer mon pays en paix" (11. 9-25). On voit donc que les pères des Hanéens sont les rois des Hanéens, et il s'agit sans doute des chefs de sept des huit ou neuf tribus hanéennes. La même alternance entre "roi" et "père" se trouve dans le second groupe des rois de l'Assyrie. On y lit en effet "dix rois qui sont des pères". 402 En outre, le père de Rīm-Sîn, le roi de Larsa (18221763 avant notre ère), était appelé "père de Emutbala (la tribu Yamutbal)". Après lui d'autres rois ont porté eux aussi ce titre. ${ }^{403}$ L'appellation "pères" se trouve aussi dans un mémorandum du temps de Zimri-Lim qui traite des affaires du haut

\footnotetext{
${ }^{401} \mathrm{La}$ traduction est basée sur une comparaison entre 11. 7-8 et M.2802.IV.4.

402J. Gelb, JNES XIII (1954), p. 210.

${ }^{403}$ Cfr. $C A D$ AI, p. $72 \mathrm{~b}(4 \mathrm{a} 2$ ').
} 
pays: "au sujet de l'affaire des pères des Ḩanéens" (A.3625.10; M.13705. 6'). Il s'agit ici aussi sans doute des rois des Hanéens. ${ }^{404}$ Les rois des Hanéens du temps de Yaḩdun-Lim possédaient un pays (M.2802.IV.3; IAHD. 20), et ils ont construit une ville qui était leur centre tribal. A la tête de cette ville se tenait un des pères des Hanéens du nom de Kașūri-Ḩala, qui portait le titre de roi. Il semble qu'à la suite de la conquête du pays des Hanéens Yaḩdun-Lim s'attribua le titre de "roi de Mari et du pays des Hुanéens" 405 ou "roi de Mari, de Tuttul et du pays des Ḩanéens". 406 Quant à Zimri-Lim, il portait le premier des deux titres. ${ }^{407}$ En outre, Bahdi-Lim, le préfet du palais de Mari, écrit à Zimri-Lim: "Si tu es le roi des Hanéens, tu es aussi, secondement le roi des Accadiens" (VI.76. 20-21).408 Dans le cas de Zimri-Lim, à la différence des vrais rois des Hanéens, il ne s'agit pas d'un roi d'une tribu hanéenne, mais d'un roi dont la population est en grande partie nomade. Le titre "pères" se trouve aussi joint au nom du pays d'Idamaraș. L'employé Bannum écrit à Zimri-Lim: "Et écris donc aux pères d'Idamaraș et à Adūna-Addu, pour qu'ils viennent vers toi ... Auparavant, Yahdun-Lim, quand il se rendit dans ce pays, fit des cadeaux aux pères d'Idamaraș" (AREP., p. 109, 11. 21-22, 27-28). Le titre "pères" montre que nous avons affaire ici à des chefs tribaux. Nous avons vu qu'il y avait dans l'Idamaraş une forte présence des Hanéens, d'où la conclusion que ce sont les rois ou pères des Hुanéens qui habitaient l'Idamaraș. 409

B. Les rois des Bini-Yamina - Les rois des Bini-Yamina apparaissent pour la première fois dans la grande inscription de Yahdun-Lim, qui relate l'insurrection de trois rois des Bini-Yamina: Lā'ûm, le roi de Samānum et du pays des Uprapûm, Baḩlu-kulim, le roi de Tuttul et du pays d'Amnanûm, et Ayālum, le roi

\footnotetext{
${ }^{404}$ F. Joannès, Miscellanea Babylonica, p. 108.

405 M.2802.I.19.

406 IAHD.3-5.

407EMPR., p. 253: I.6-7; II.4-5; IX.33 (Sc.); 36 (Sc.); OBTR , p. 250, Sceau 5, 11. 6-7 et voir

D. Charpin et J.-M. Durand, RA LXXX, pp. 149-150.

${ }^{408}$ Cfr. aussi M.9649.

409J.-R. Kupper, Nomades, p. 33; F. Joannès, Miscellanea Babylonica , p. 108.
} 
d'Abattum et du pays des Rabbûm, contre Yahdun-Lim (M.2802.III.3-27). Cette insurrection s'est terminée par une victoire décisive de Yahdun-Lim qui a donné son nom à l'une de ses années de règne: "L’année où Yahdun-Lim a infligé une défaite aux Bini-Yamina" (VIII.75.21-23). A la suite de cette défaite on ne trouve plus de rois des Bini-Yamina dans le royaume de Yahdun-Lim. Pendant le règne de Samsi-Addu nous n'entendons pas parler des rois des Bini-Yamina, et c'est seulement durant le règne de Zimri-Lim que nous entendons parler d'eux à nouveau. Parmi les trois villes-royaumes bini-yaminites du temps de YahdunLim, seule Abattum est devenue de nouveau une ville des Bini-Yamina sous l'autorité du roi des Rabbûm (XIV.83.36-37). Au début du règne de Zimri-Lim, Samānum elle aussi est redevenue une ville-royaume bini-yaminite du roi biniyaminite Samsi-Addu.410 Puis après la victoire de Zimri-Lim sur les BiniYamina, les villes de Samānum et de Tuttul devinrent les sièges des préfets (II.137.30, 35, 39, 45, 47; III.73.9). Voyons maintenant ce que nous savons à propos des rois des Bini-Yamina. D. Charpin et J.-M. Durand fournissent l'information suivante les concernant. ${ }^{411}$ On connaît les noms de plusieurs rois BiniYamina du début du règne de Zimri-Lim: Hardûm, Sūmu-dabi, Samsi-Addu, et Yagih-Addu. Yagih-Addu était le prince (probablement une traduction de LUGAL = roi) de la ville de Mišlān (XXVI.170.22'-23') du district de Mari, Samsi-Addu celui de Samānum, Sūmu-dabi s'est marié avec une soeur de Zimri-Lim ${ }^{412}$ et Yagih-Addu s'est marié avec sa fille. ${ }^{413}$ Les rois de Bini-Yamina se considéraient comme "frères". 414 Par la suite, les relations entre Zimri-Lim et les rois des Bini-Yamina se sont détériorées et une guerre a éclaté entre eux. L'année ZL 2' porte le nom: "L'année où Zimri-Lim a infligé une défaite aux Bini-Yamina à Saggarātum et a tué (ou: capturé) leurs rois" (N.AN., p. 55, no. 6; XXI.128. 13).

\footnotetext{
${ }^{410} \mathrm{XXVI} / 1$, p. 340, n. 26. lîbûr" dans XXVI/3), 339-340.

412XXIII.335 (4.XI.ZL 1) et 336 (1.XII.ZL 1).

${ }^{413}$ D. Charpin et J.-M. Durand, $R A$ LXXX, p. 172, n. 145.

${ }^{414}$ XXVI.168.18; 170.23'; 171.11-12, 14', 16'; 172.7, 13, 29.
}

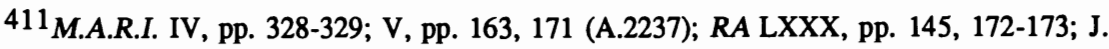
Sasson, Miscellanea Babylonica, pp. 253-255; XXVI, pp. 139 ("La correspondance de Dâriš- 
L'événement lui-même a eu lieu pendant l'année ZL 1'. Il nous semble que cette guerre est liée à un rêve dont le contenu a été raconté à Zimri-Lim par Itūr-Asdu, qui était le préfet du palais de Mari pendant les premières années de Zimri-Lim: "Dagān prit la parole et il me dit ceci: Les rois des Bini-Yamina et leurs armées, ont-ils fait la paix avec l'armée de Zimri-Lim qui est montée ?" (XXVI. 233.1621).415 A la suite de la victoire de Zimri-Lim les rois des Bini-Yamina se sont réfugiés à Qatanum (Samsi-Addu), Karkamiš (Yagihn-Addu [?]) et au Zalmaqum.(*) Amud-pī-El, le roi de Qatanum, a chassé Samsi-Addu de chez lui. Ensuite les rois des Bini-Yamina ont été aidés par Ešnunna. On peut supposer qu'à l'issue de tous ces événements dramatiques, de nouveaux rois des BiniYamina apparaissent sur la scène. Ils ont créé des villes-royaumes dans la région d'amont, une région qui était hors de la portée de Zimri-Lim.

Nous avons vu qu'Itūr-Asdu a informé Zimri-Lim à propos du conflit entre celuici et les Bini-Yamina. Or, un autre missive d'Itūr-Asdu nous apprend qu'un danger venant des rois bini-yaminites menace Zimri-Lim qui est en route vers Mari (A.82). Enfin, Itūr-Asdu informe Zimri-Lim d'une victoire de Yasīm-Mahar sur les rois des Bini-Yamina dans la région d'amont (BENJ., pp. 992-993a). Il est possible qu'à la suite de cette victoire Șūra-hammu informe Zimri-Lim que "tous les Bini-Yamina sont retournés au pouvoir de mon père; du plus petit au plus grand, ils appartiennent en vérité à mon père" (BENJ., pp. 993b). Après la victoire, Yasīm-Maḩar est venu avec des représentants des Bini-Yamina à Mari pour conclure une alliance avec Zimri-Lim (III.50). Durant l'année ZL 4' des prisonniers et des prisonnières des Bini-Yamina ont été libérés par leurs familles, peut-être à la suite de la visite de Șūra-ḥammu, le roi d'Amnanûm, à Mari. ${ }^{416} \mathrm{La}$ guerre des Bini-Yamina contre Zimri-Lim, qui éclata après la montée de ZimriLim sur le trône de Mari, avait pour but de reconquérir les régions qui étaient sous leur domination avant leur défaite face à Yahdun-Lim. Après la victoire de Zimri-Lim sur les Bini-Yamina et la conclusion de l'alliance entre eux, ils sont devenus des vassaux de Zimri-Lim comme Șüra-hammu, le roi d'Amnanûm, et Dādi-ḩadun, le roi des Rabbûm, qui appellent Zimri-Lim "père", tandis qu'ils sont

${ }^{415}$ Cfr. aussi XXVI.199.

416 Voir XXIII, pp. 21, 494. 
ses "fils" (II.61.1-4; BENJ., p. 993a). Les titres "père" et "fils" montrent qu'il s'agit de relations "Souverain" - "Vassal".417 Par contre les relations de Hुammiištamar, le roi d'Uprapûm, et Yasmah̆-Addu, le roi des Yarihûum, avec Zimri-Lim étaient sur la base de "Seigneur" - "Serviteur" (II.53.1-4; 54.1-4 ;55.1-4; AREP., pp. 111-112). Ici aussi nous avons affaire à des relations "Souverain"- "Vassal", mais vassal d'un niveau plus bas. Une lettre de Hammi-ištamar à Ibal-El fait allusion, pensons-nous, à une alliance que celui-ci a conclue avec Zimri-Lim, lors de la visite de Zimri-Lim à Ugarit (A.2094.9-10). Quand Dādi-ḩadun s'adresse à la reine Šibtum, il n'emploie aucun titre: "Dis à Šibtum: ainsi (parle) Dādi-ḩadun" (X.156.1-2). Par contre Šibtum se fâche contre Yasmah-Addu parce qu'il emploie le titre "serviteur" quand il s'adresse à elle (X. 159.1-10).

Le regroupement de toute l'information sur les cinq rois des Bini-Yamina: Dādihadun, le roi des Rabbûm (XVI/1, p. 84; XXI.219.50'-51'), Ḩammi-ištamar, le roi d'Uprapûm (XIV.87.8-9), Șūra-ḩammu, le roi d'Amnanûm (XVI/1, p. 189), Yarìm-Lim, le roi des Yahrurûm (II.93.6'-7'), et Yasmah-Addu, le roi des Yarihûm (XVI/1, p. 231), qui se trouve dispersée dans les textes de Mari peut nous éclairer sur la signification du titre dans une tribu semi-nomade. Le tableau qui suit est un tableau chronologique attestant la présence de ces rois dans les textes de Mari:

${ }^{417}$ Pour les différents degrés de vassalité cfr. XXVI.384.63'-65': ahūtum et mânūtum. 
AnnéelDadi-ḩadun Ḩammi-ištamar Şūra-ḩammû Yarim-Lim Yasmaḩ-Addu

1

Kahat

1' (Année de la victoire sur les Bini-Yamina)

2 '

3'

4

5 ,

$6^{\prime}$

7

$\begin{array}{llllll}8^{\prime} & + & & + & ? & \\ 9^{\prime} & + & ? & & + & \\ 10^{\prime} & + & + & + & + & + \\ 11^{\prime} & + & + & + & + & + \\ 12^{\prime} & & & & + & +\end{array}$

Il existe seulement un document (et son duplicata) où figurent ensemble les cinq rois des Bini-Yamina (XXIII.428-429). Il s'agit d'une liste de recensement de supplétifs (warkûm) des Bini-Yamina, qui s'est déroulé à Dīr, au sud de Mari, l'année ZL 10'. La liste est divisée en quatre colonnes et six paragraphes. Dans la première colonne apparaît le quota que le district était obligé de fournir, dans la seconde colonne, le nombre des hommes qui sont effectivement venus, dans la troisième colonne, le nombre des manquants, et dans la quatrième et dernière colonne sont enregistrées (par districts) les villes d'origine de ces gens. Quant aux paragraphes, le premier énumère les gens de Yarīm-Lim des localités dans les districts de Mari, de Terqa et de Saggarātum (XXIII.428.2-10), dans le second, les gens de Yasmah-Addu des mêmes districts (11. 11-18), dans le troisième, les gens de Șūra-ḩammu de Terqa et de Saggarātum (11. 19-25), dans le quatrième, les gens de Dādi-hadun des districts de Terqa et de Saggarātum également (11. 26-30), et le cinquième, les gens de Ḩammi-ištamar du district de Terqa (11. 31-36). La sixième colonne donne le résumé (11. 37-42). Ce document montre que les localités des Bini-Yamina des districts de Mari, de Terqa et de Saggarātum étaient divisées d'après les cinq tribus des Bini-Yamina et leurs habitants, et dépendaient dans une certaine mesure des rois de ces tribus. Il faut souligner que les rois habitaient 
dans la région d'amont hors de la juridiction de Zimri-Lim, tandis que leurs villes se trouvaient dans le coeur même du royaume de Zimri-Lim. Sur l'attitude de ces villes envers Zimri-Lim d'une part et envers les rois des Bini-Yamina d'autre part nous sommes informés par plusieurs lettres. Dādi-ḩadun écrit à Zimri-Lim: "A Zimri-Lim, mon père, dis ceci: ainsi (parle) Dādi-ḩadun, ton fils. Toutes les villes [appartiennent à mon seigneur] et nous sommes tes fils, et toi, tu sais (que), dans ce pays, [les fils] de mon [seigneur] occupent de très nombreux villages" (II.61.19). Nous lisons des paroles semblables dans la lettre de Yasmah-Addu: "Les villes - terre et cieux - sont à mon seigneur seul. Mon seigneur a libéré ces villes" (II.55.10-11). On peut comparer les expressions des rois des Rabbûm et des Yarihûm avec les paroles de Hiāya-Sūmû roi d'Ilānșūra, qui était lui aussi un vassal de Zimri-Lim: "Tu (Zimri-Lim) m'as écrit que tu as écrit avec insistance à Atamrum (le roi d'Andarig) au sujet de la libération de mes villes ... Ces villes sont tes villes" (II.62.4-6). De même, Išhi-Addu, le roi de Qatanum, écrit à ZimriLim: "Cette maison est ta maison !" (V.20.25). Dans une autre lettre, Yasmah̆Addu, le roi des Rabbûm, écrit à Zimri-Lim à propos de trois villes que ZimriLim lui a données, et il termine sa lettre avec les paroles suivantes: "dans mes villes je n'ai pas laissé un seul homme" (II.56.19-20). Une ville appartenant à Șūra-ḩammu est mentionnée dans un passage fragmentaire d'une lettre de KibriDagān: "dans ma ville" (III.36. 9). (*)

Nous avons vu que Zimri-Lim octroie des villes aux rois des Bini-Yamina, mais parfois il enlève des villes à ces rois, et alors les sugāgū de ces villes s'adressent à leurs rois en leur demandant d'aller chez Zimri-Lim pour réclamer ces villes (II.53). 418 Et Șūra-Hammu, le roi d'Amnanûm, demande à Zimri-Lim de ne pas confisquer les champs et les maisons de membres de sa tribu, appelés "fils du fleuve" mārū nārim, qui vont descendre chez eux dans l'Ah̆-Purattim (A.1281). Un trait de ressemblance entre les rois vassaux de Zimri-Lim d'Idamaraş et les rois des Bini-Yamina se trouve dans le fait que le territoire gouverné par Hammiištamar est appelé "district" (II.94.25), un terme qui se réfêre par exemple au pays de Kabiya, le roi de Kahat, qui était un vassal de Zimri-Lim (II.59.5). A propos du roi bini-yaminite du début du règne de Zimri-Lim, Sūmu-dabi, nous savons

${ }^{418}$ Cfr. aussi M.6874.4'-5' et M.8436.Rev. 
qu'il avait un palais dans sa ville-royaume (XXVI.169.4'), son collègue Yagih̆Addu avait à Mišlān 1000 ou 2000 soldats (XXVI.171.27'), des forces de gendarmerie bazahātum, ${ }^{419}$ et des devins. ${ }^{420}$ Les autres rois bini-yaminites avaient aussi à leur disposition des armées (XXVI.171.16'-18'); enfin il faut noter que Sūmu-dabi demande aux serviteurs de Yagih-Addu: "envoie-moi un boeuf, que je fasse le sacrifice" (XXVI.170.16'). A en juger par la correspondance entre les devins Yamşi-ḩadnû, Mašum, Ḩammi-Esim et le roi Sūmu-dabi (XXVI.168-172) on peut arriver à la conclusion que la royauté bini-yaminite, au moins celle du début du règne de Zimri-Lim, n'était pas différente de celle de Zimri-Lim ou des autres rois de l'époque.

Un document juridique qui traite d'un acte de garantie (VIII.67) nous révèle le statut de Șūra-ḩammu. Le garant est un cultivateur de Șūra-ḩammu habitant de Zarri-Amnān (11. 3-5), un des témoins est le vizir de Șūra-ḥammu (11.8'-9'), et un autre est son messager (11. 10'-12' et cfr. M.8990.1-2). Șūra-hammu, le roi d'Amnanûm, possède un cultivateur (X.151.11-12) et un jeune serviteur personnel (II.104.5). Șūra-ḩammu intervient auprès de Zimri-Lim dans une affaire qui concerne un champ de la ville de Zarri (XXVI.74.19-27). Un serviteur de Șūra-ḩammu du nom de Yawi-Erah reçoit un habit du Palais (XXI.339.1-5; 370.2'-4'). Yasmah-Addu, le roi des Yariḩéens, possède lui aussi un jeune serviteur personnel (II.55.34). Dādi-ḩadun, le roi des Rabbéens, quant à lui, avait sous ses ordres un vizir (XXV.136.5) et un merhum (XXVI.114). (*) Les rois des Bini-Yamina possèdent des bateaux: Șūra-ḩammu monte sur un bateau qui va à Zarri-Amnān (XIV.83.7-8) et reçoit un anneau de fer sur d'autres bateaux (XXV.117).421 On prélève des taxes sur les bateaux d'un autre roi, Hammiištamar, le roi des Uprapéens (XIII.86.11-12). Șūra-ḩammu, Yasmah̆-Addu et Yarìm-Lim envoient du bétail à Mari, comme faisaient les rois vassaux, les gouverneurs et les ministres (VII.226.33; XXIII.222.7-8; XXIV.31). Dãdi-ḩadun et Yarīm-Lim envoient au Palais des gobelets, des objets en métal et des vêtements (XXI.252.8-11; XXIII.542.35-36; XXV.38+). De son côté le Palais

\footnotetext{
${ }^{419}$ XXVI.168.35; 169.4'; 172.22 .

${ }^{420}$ XXVI.168.38-39; 169.6-8, 9'-14'; 170.6-17; 171.8'-14'.

${ }^{421}$ Cfr. aussi M.8436.14.
} 
donne à Șūra-haammu des vêtements, un arc, une outre et un anneau en métal.422 Dādi-ḩadun reçoit un arc (XXIII.455.3-4), Hammi-ištamar (?) reçoit, quant à lui, une selle d'âne (XVIII, p. 109, S.143, no. 132), Yasmah̆-Addu reçoit huit sicles d'argent et cinq moutons (IX.23). Un dirigeant uprapéen du nom de Pulhu-Addu reçoit lui aussi des vêtements (XXIII.15.11-15; 16.12-15; 18.12-16) quant à Yasmah-Addu il a emprunté de l'argent à Zimri-Lim (VIII.37; 42). Et enfin, il se peut qu'une fille de Zimri-Lim ait été donnée en mariage à Dādi-ḩadun (XXI.219; M.12074).

Les rois avaient l'habitude de faire des visites à Mari, ${ }^{423}$ où ils s'asseyaient devant Zimri-Lim (III.70.4'-5' (*); XXVI.74.21-22). Les rois rendent aussi visite aux gouverneurs des districts: Șūra-ḩammu visite Terqa (III.58.11-18; XII. 132. 7-8) et le district de Saggarātum (II.105). Dādi-ḩadun visite Terqa (III. 45.9-16; XIII.123.12-20), Ḩammi-ištamar visite Saggarātum (XIV.87), et Yarīm-Lim va du district de Terqa au district de Saggarātum (III.93.1'-9'). (*)

Les rois habitaient dans la région d'amont, et pour cette raison on dit que Șūrahammu descend à Terqa (III.58.11-12) ou monte sur un bateau et navigue vers Zarri-Amnān, qui se trouve dans le district de Saggarātum (XIV.83.7-8).

Nous ne savons pas comment les rois ont été élus, mais pourtant nous avons un indice qui prouve que la tribu reconnaissait l'autorité du roi. La tribu Yarihûm déclare: "A part Yasmah-Addu, le roi, notre seigneur, nous ne connaissons pas d'autre roi" (AREP., p. 112a). ${ }^{424}$ Mais l'autorité du roi sur sa tribu n'est pas absolue, comme nous l'apprend la lettre de Yaqqim-Addu: "... et tous les BiniYamina sont préoccupés, Hammi-ištamar ne cesse de leur écrire, mais personne ne va chez lui" (XIV.83.23-26). L'attitude des Bini-Yamina envers leur roi rappelle leur attitude envers le gouverneur Kibri-Dagān. Ils refusent de venir chez lui, à son appel, pour l'aider lors de la moisson (III.38.24-26).

Comme nous allons le voir plus loin, des sugăgū se tenaient à la tête des villes et des unités tribales. Quelles étaient les relations entre les rois et les sugāgū?

\footnotetext{
${ }^{422}$ XXI.370.2'-4'; XXIII.12; 15.1-10; 16.1-10; 18.1-11; 19; 455.1-2; XXV.117. (*)

423II.53.27-29; 54.5'-11'; 61.28-29; XIV.93.13-15; XXIV.72 et voir XXIII, pp. 17-21. ( $\left.{ }^{*}\right)$

${ }^{424}$ En supposant, bien sûr, qu'il ne s'agisse pas de Yasmah-Addu fils de Samsi-Addu.
} 
Une lettre de Yasmah-Addu, le roi des Yarihum, (II.53), nous informe que les sugāgū sont venus trouver les rois bini-yaminites à Ahunā, sur le Balịh, et leur demandent d'aller chez Zimri-Lim pour réclamer leurs villes qui se trouvent probablement dans l'Ah-Purattim, et qu'on leur a enlevées. On peut, peut-être, se référer ici aussi à une lettre d'Asqudum et Rišiya où est cité un discours adressé aux rois du Zalmaqum, mais à cause d'une cassure dans la tablette on n'en connaît pas les auteurs. Nous pensons qu'ils s'agit de membres de la tribus BiniYamina, qui disent: "Allons, envoyons nos rois ou nos sugāgū afin qu'ils discutent avec vous" (XXVI.12.3'-5'). Puis, c'est Yarīm-Lim, le roi de H̆alab, qui évoque la possibilité qu'ils ne seront pas représentés dans les négociations par leurs rois mais par leurs sugāgū" (11. 8'-10'). C'est dans le même contexte que doit être comprise la fameuse lettre d'Ašmad, qui traite de l'alliance conclue dans le temple de Sîn à Harrān. Les trois rois des Bini-Yamina du début du règne de Zimri-Lim, Yagih̆-Addu, Ḩardûm et Samsi-Addu sont entrés à Aḥunā, sur le Balīh, mais ce sont les sugāgū et les Anciens des Bini-Yamina qui représentent la tribu lors de la conclusion de l'alliance avec le roi de Harrān et les rois du Zalmaqum (XXVI.24.6-12). Ici on doit intercaler l'information d'après laquelle les rois des Bini-Yamina étaient invités par les rois du Zalmaqum pour se rencontrer à Zalpah en vue d'un palabre, sans doute afin de conclure une alliance entre eux (A.2526).

Deux documents nous montrent que les rois des Bini-Yamina ont eu une certaine autorité sur les membres de leurs tribus qui habitaient en dehors de la zone de leur juridiction. Le premier document (et sa copie) enregistre le résultat d'un recensement à Dïr des Bini-Yamina habitant les districts de Mari, de Terqa et de Saggarātum, avant qu'ils ne soient envoyés en Babylonie. Or, ces Bini-Yaminites sont divisés d'après les rois des cinq tribus des Bini-Yamina (XXIII.428-429). Le second document traite d'un cas où Kibri-Dagān, le gouverneur de Terqa, était obligé de fournir au roi de Mari trois cents vêtements. Kibri-Dagān demande à Zimri-Lim de s'informer auprès des rois des Bini-Yamina qui se trouvent chez Zimri-Lim si les villes des Bini-Yamina doivent fabriquer ces vêtements (III. 


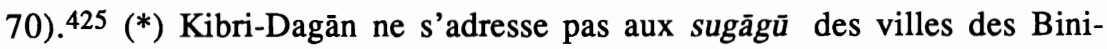
Yamina, mais bien aux rois des Bini-Yamina. On voit donc que l'autorité des rois s'étend aussi sur des localités où habitent les membres de leurs tribus dans le coeur même du royaume de Zimri-Lim. Ce fait peut expliquer le cas d'un conflit qui éclata entre Kibri-Dagān, le gouverneur de Terqa, et Ṣūra-ḩammu, le roi amnanéen. La lettre dans laquelle Kibri-Dagān relate l'affaire à Zimri-Lim nous montre, en outre, qu'au moins dans son district, Kibri-Dagān jouissait d'une autorité plus élevée que celle de Șūra-ḩammu. Mais ce n'est pas le cas pour Yaqqim-Addu, le gouverneur de Saggarātum, qui donne l'impression d'avoir peur de Șūra-ḩammu (XIV.83.9-13).

Les rois, à l'instar des gouverneurs, étaient obligés de fournir, comme nous venons de le voir, des contingents au roi de Mari (XXIII.428-429 et voir aussi II.56 et 53.5).

Nous trouvons un important témoignage quant aux relations entre Zimri-Lim et les rois des Bini-Yamina dans une lettre de Șūra-ḩammu l'Amnanéen à Dādihadun le Rabbéen (A.3821.14-18). Dans cette lettre Șūra-ḩammu se plaint du fait que "ici (à Mari) Yarīm-Lim (le roi des Yaḩrurûm) s'est présenté devant ZimriLim pour dire du mal de ton (Dãdi-ḩadun) serviteur (Ṣūra-ḩammu), et sur son ordre on a emprisonné ton serviteur". On voit donc que Zimri-Lim possédait le pouvoir même d'emprisonner ses vassaux bini-yaminites; en outre on constate qu'il existait une hiérarchie entre ces rois, car Șūra-ḩammu se qualifie de serviteur (littéralement "esclave") de Dādi-ḩadun (1.18); enfin cette lettre atteste aussi une période de mauvaises relations entre Șūra-ḩammu et Yarīm-Lim (11.19-20), à la différence de l'époque de coopération attestée par la lettre II.53. J.-M. Durand rapporte ainsi le contenu d'une lettre (A.730) envoyée par Șūra-ḩammu à HabduMalik: "Le prince benjaminite se plaint de ce qu'on le calomnie devant le roi, alors qu'il tient une zone frontière, exposée. Il n'a que Habdu-Malik pour plaider sa cause. Il lui demande que l'on fasse taire les calomniateurs et d'améliorer son image de marque auprès du roi." 426

${ }^{425} \mathrm{Cfr}$. les deux listes de vêtements fabriqués dans le district de Terqa (IX.97; 102) et une dans le district de Saggarătum (XXII.121). (*)

${ }^{426} \mathrm{XXVI} / 1$, p. 385. 
Des vicissitudes dans les relations entre Yasmah-Addu, le roi des Yarihéens, et Lanasûm, le préfet de Tuttul, nous sont révélées par deux documents. Dans une lettre de Lanasûm nous lisons que Yasmah-Addu en collaboration avec Abum-El, un percepteur de l'administration de Zimri-Lim, et Yašūb-El, un notable de Tuttul, voulait inciter les habitants de Tuttul et les Bini-Yamina des environs de Tuttul à évincer le préfet Lanasûm (II.137). Par contre, dans l'année ZL 7' nous trouvons le même Lanasûm parmi les témoins dans un document de prêt fait par le palais à Yasmah-Addu (VIII.42). Enfin, par une lettre du commandant des troupes hanéennes à Mari nous apprenons que Șūra-ḩammu a envoyé une expédition pour piller les biens d'un certain Asqudum, qui s'est noyé (M.8436.4-8).

Sous l'autorité des rois des Bini-Yamina on trouve aussi des muškēnū, qui représentent la couche de la population qui ne fait pas partie de la société tribale, les "Accadiens" d'après la terminologie de Bahdi-Lim, le gouverneur de Mari (VI. 76.21). Yasmah̆-Addu rapporte à Zimri-Lim les complaintes des muškēnū : "Mon seigneur (Zimri-Lim) a affranchi quatre villes désertiques, et ainsi on a repoussé mon pouvoir dans quatre villes en Ara'itum (dans le district de Mari), et je ne peux pas répondre aux muškēnū qui disent: les villes de tes frères (les Yariḩéens) et leurs champs sont francs; mais toi, tu as livré nos champs au palais !" (II.55.26-32). Nous supposons qu'il s'agit du cas suivant: les Yarihéens tenaient en fermage des champs et des villes qu'ils avaient reçus du palais, et Zimri-Lim ordonna de les affranchir afin qu'ils deviennent leur propre bien. A la place de ces champs et villes Yasmah̆-Addu a octroyé au palais les champs des muškēnū. La juridiction de Zimri-Lim sur les villes des Yarihûm rappelle le cas où les dirigeants des Bini-Yamina vont chez Zimri-Lim pour lui demander de leur rendre leurs villes (II.53). Une situation opposée à celle qui est décrite dans la lettre de Yasmah-Addu nous est rapportée dans une lettre d'un autre roi des Bini-Yamina, Dādi-hadun, le roi des Rabbûm (II.61.1-27). Il semble d'après cette lettre que les muškēnū et le palais jouissaient d'une réduction des taxes ${ }^{427}$ perçue sur leurs champs par rapport aux Rabbûm. Peut-être faut-il faire allusion dans le même

${ }^{427}$ Nous proposons de comprendre ainsi le verbe gurrum. $C A D$ G, p. $140 \mathrm{~b}$ traduit "to allot (fields to settlers)" et $A H w$, p. 287a quant à lui traduit "anfordern". 
contexte à une lettre de Yarim-Lim, le roi des Yahrurûm, à la reine Sibtum, ${ }^{428}$ X.151. Dans cette lettre Yarim-Lim parle d'un champ qui lui appartient et qui est voisin des champs des muškēnū, 11. 18-20.

C. Un roi des Numḥāa - Simaḩlānē, qui appartenait à la tribu Numhāa (X. 5.4), monta sur le trône de Kurdā, avant Bunu-Ištar et Hammurabi (de Kurdā). La prise de Kurdā par Simahlānē se réalisa probablement avec l'aide d'un contingent babylonien, comme nous l'apprennent une série de documents qui relatent son arrivée à Mari en route vers Kurdã à la tête de 150 Babyloniens et 50 Numhéens. 429

D. Conclusion - A la lumière des données que nous avons rassemblées sur les pères (ou: rois) des Hanéens et les rois des Bini-Yamina il nous semble qu'on peut conclure qu'ils étaient les chefs des tribus des Hanéens et des Bini-Yamina. ${ }^{430} \mathrm{~L}$ 'institution de la monarchie tribale ne pouvait pas exister dans les limites mêmes du royaume de Mari. Après la victoire de Yahdun-Lim sur les rois des Hanéens et les Bini-Yamina il semble que les Bini-Yamina n'ont plus eu de rois, au moins dans le royaume de Mari, car Yahdun-Lim contrôlait la région d'amont, le pays où l'on trouve la principale concentration des Bini-Yamina. Par contre, les pères des Hanéens continuaient à régner dans l'Idamaraș, car ce territoire se trouvait en dehors de la domination de Yahdun-Lim. A l'époque du royaume de Samsi-Addu il est très probable que la monarchie tribale n'existait pas, car SamsiAddu contrôlait aussi bien l'Idamaraș que la région d'amont. Par contre, à l'époque de Zimri-Lim, les rois et les pères réapparaissent. Au début de son règne, Zimri-Lim a remporté une victoire sur les rois des Bini-Yamina, et par la suite on ne les trouve plus que dans la région d'amont. Les Hanéens eux aussi renouve-

${ }^{428}$ Sur les rapports entre Sibtum et un autre roi des Bini-Yamina, Dādi-hadun, le roi des Rabbûm, cfr. XXI.219.50'-51'.

${ }^{429}$ SIMA., p. 132; A.2983; A.2830; A.2801; XII.29; A.826.

${ }^{430}$ A l'instar de Zaziya qui était le roi de la tribu hurrite Turukkûm (XXI.333.25'). Et encore, dans une lettre de Sušarră on parle d'un roi des Turukkûm qui règne dans une ville (J. Eidem, Iraq XLVII [1985], p. 91). 
laient la monarchie (comme à l'époque de Yaḩdun-Lim), et on trouve des pères des Hanéens en Idamaraş, comme au temps de Yahdun-Lim. Les rois des BiniYamina étaient des vassaux de Zimri-Lim tandis que les pères des Hanéens étaient indépendants.

Il est fort probable qu'à l'époque de Zimri-Lim, comme à l'époque de YahdunLim, les rois des Bini-Yamina régnaient dans des villes-royaumes, possédant une cour royale comme tous les autres souverains de l'époque. $\left(^{*}\right)$ Il faut aussi souligner que les rois des Bini-Yamina avaient un certain pouvoir sur les localités des Bini-Yamina qui se trouvaient dans le coeur même du royaume de Zimri-Lim, et même dans des localités où vivaient les muškēnū. Au début du règne de Yahdun-Lim chacune des deux confédérations tribales possédait une ville qui servait de centre tribal. Le centre des Hanéens était la ville de Haman, et le centre des Bini-Yamina était la ville de Samānum. A l'époque de Zimri-Lim, les villes de Zalpaḩ et de Ahunā remplissaient peut-être cette fonction (II.53).

\section{Le madārum}

Le titre madārum se trouve seulement dans les documents de Mari. Il est fort probable que nous ayons affaire à un mot d'origine amurrite. D'après les contextes dans lesquels on trouve ce terme, on peut conclure qu'il signifie "vassal".431 Dans une lettre de Yaqqim-Addu, le gouverneur de Saggarātum, nous lisons un rapport de Yatarum de Dīr, qui se trouve dans "le pays d'amont", à propos d'un madārum de la tribu Uprapûm: "Quant au madārum uprapéen qui réside à SubatŠamaš, je l'ai fait partir et je l'ai amené" (XIV.88.14-17). Il est fort probable que le madārum dont parle cette lettre soit Lahun-Dagān, l'uprapéen, dont le sugāgū exigeait qu'il parte chez Zimri-Lim (II.53).

Șūra-Hुammû, le roi d'Amnanûm, a écrit à Dādi-ḩadun, le roi des Rabbûm, que "P[N/là-bas] a fait prendre Saḥbarum, le bédouin (mār h̆abbātim) de la steppe (nawûm) en vue de la madārūtum" (A.3821.10-13). Or, d'après un document économique du début du règne de Zimri-Lim (9.XI.ZL 1) nous apprenons que Sah̆barum, sans doute le même, a conduit au palais de Mari des ovins appartenant aux

${ }^{431}$ Pour J.-M. Durand, XXVI/1, p. 283 (A.3096.18) il s'agit de "noble". 
DUMU-MEŠ Salsalim (XXIII.337). Il nous semble fort probable qu'il ne s'agit pas ici de "fils de Salsalum" mais bien de "membres d'une unité tribale Salsalum".

\section{Le šāpitum}

Une liste de moutons présente les moutons comme appartenant au šāpițum de Hanûm, mais peut-être faut-il accepter la lecture de la première édition: ša pidim, quel que soit son sens. ${ }^{432}$

\section{Les dirigeants sutéens}

On ne connaît pas le terme original pour désigner un chef de Sutûm, mais les textes de Mari nous font connaître les noms de plusieurs chefs sutéens. Dans une lettre du temps de Yasmah-Addu on lit que deux mille Sutéens ont razzié le nawûm de Qatanum. A la tête de la razzia se tiennent trois chefs: Gazizānum, AbiSarē et Hammi-talû (V.23.5-13). Abi-Sarē et Hammi-talû apparaissent dans une lettre de l'époque de Zimri-Lim, où on parle des membres de la tribu sutéenne Mihalizayû qui ont trouvé un trésor au mont Bisir (VI.44). Dans cette lettre nous trouvons un autre chef sutéen du nom de Rabi'um. ${ }^{433}$ Hammi-talû et un autre chef sutéen du nom de Yapahum se tiennent à la tête d'un hibrum, une compagnie, d'une ou de plusieurs centaines de Sutéens (A.2801.14-16). Dans une autre lettre, Bahdi-Lim raconte qu'il a fourni à Hammi-talû 4200 litres de blé, sans doute une provision pour les membres de sa tribu (VI.15.18-22). Au temps de Yasmah-Addu ce même Hammi-talû doit conduire une caravane de marchands de Mari à Qatanum (I.17.36-43). Dans une liste de payement aux messagers de l'époque de Zimri-Lim on trouve les chefs sutéens Hammi-talû et Rabi'um (VII.210.19'-20'), et dans une autre liste du même genre apparaît Rabi'um seul

${ }^{432}$ Voir M.A.R.I. V, p. 643 (p.3) où nous avons proposé la lecture šapiţum.

${ }^{433}$ Rabi'um est un nom propre et non pas un nom de tribu comme l'a supposé Sh. Arbeli (M. H. Helzer-Sh. Arbeli, The Suteans, Naples 1981, pp. 14-17 et voir la remarque de J.-R. Kupper, ibidem, p. 14, n.8). 
(VII.165.15). Il faut se rappeler que celui qui porte le titre "messager", mār šiprim, n'est pas seulement chargé de transmettre des renseignements, mais il pouvait aussi remplir des missions diplomatiques ou commerciales: un vrai chargé d'affaires. ${ }^{434}$ Il arrive aussi que Rabi'um envoie, avec Zannum, un autre chef sutéen, ${ }^{435}$ des messagers (VII.133.1-6). Hammi-talû et Rabi'um, à l'instar des vassaux et de hauts fonctionnaires du royaume, envoient des moutons au palais de Mari (XXI.2; 10.2-3). Enfin il faut considérer les cas suivants: 1. Un Sutéen du nom de Rabi'a et deux autres Sutéens reçoivent un habit du palais en l'an ZL 4' (XXI.336). Le fait que Rabi'a reçoive un habit d'une meilleure qualité que ceux que reçoivent les deux autres membres de sa tribu, et que, dans la liste, il soit noté séparément d'eux, peut indiquer que nous avons affaire à notre chef sutéen Rabi'um. 2. Du fait que Ballum, le Sutéen, envoie des moutons au palais (XXI.4), on peut peut-être conclure qu'il était, lui aussi, un chef sutéen. 3. Un habit et un arc sont envoyés à un personnage dont il ne reste que la fin du nom Xhan (XXII.170.Rev.6-8). Un envoi šūbultum, différent de la simple livraison à Mari, est destiné aux gens importants.

\section{Les dirigeants numbéens}

Dans une lettre d'Abi-mēkim apparaissent les dirigeants (qaqqadātum) de Numhāā qui se joignent avec les Anciens du pays, les serviteurs de Simaḩlānē (XXVI. 463.5-8).

\section{Le sugågum}

Le mot sugāgum n'est pas un mot accadien, mais très probablement un mot d'origine amurrite en dépit du fait qu'on ne connaît pas son étymologie. Le sugāgum était soit le chef d'une localité, ville (maire) ou village (moqtar), soit le chef d'une

${ }^{434}$ Voir VII, pp. 234-235.

435Voir M.A.R.I. II, p. 80, no. 133.6 . 
unité tribale (cheik). ${ }^{436}$ Des villes importantes comme Saggarātum et Dür-Yah̆dun-Lim étaient elles aussi gérées par des sugāgū (VII.311; XIV.46). La double fonction des sugāgū, maire-moqtar et cheik, provient du fait que l'origine du terme était dans la société tribale et qu'avec la sédentarisation de la tribu le terme se référa tout naturellement au chef de la localité habitée par cette tribu, et à partir de là à toutes les localités, même celles qui n'étaient pas habitées par les membres des tribus. Le terme accadien qui correspond à sugāgum est, semble-t-il, rabiānum, rabânum "le grand", qui désigne aussi bien le chef d'une unité tribale que le chef d'une localité. ${ }^{437} \mathrm{Le}$ fait que le terme accadien n'apparaisse pas dans les textes de Mari renforce l'impression qu'à Mari on emploie à sa place le terme sugāgum.

L'alternance, dans la même lettre, entre le terme abstrait sugāgūtum, dérivé du nom sugāgum, et le terme šāpirūtum, dérivé de la racine šapārum qui signifie "diriger, dominer"438 (V.24.11-12, 20-22) permet de comprendre quelle était l'activité du sugāgum. Le sugāgum était responsable de la gestion des affaires de l'unité sédentaire ou nomade à la tête de laquelle il était placé. Les sugāgū remplissent leur fonction dans des localités habitées soit par la couche accadienne de la population soit par la couche amurrite. 439 Cette dernière pouvait être composée des sédentaires sans aucun lien tribal ou des membres des diverses tribus amurrites. On connaît des sugāgū des Hanûm ${ }^{440}$ et des sugāgū des Bina-Yamina. ${ }^{441}$

${ }^{436}$ XV, p. 255; $A H w$, p. 1053b; J.-R. Kupper, op. cit., pp. 15-17; "Les Pouvoirs locaux dans le royaume de Mari" dans Les Pouvoirs locaux en Mésopotamie et dans les régions adjacentes, A. Finet (éd.), Bruxelles 1982, pp. 50-52; H. Klengel, Benjaminiten und Hanäer, p. 219; A. Finet, Akkadica XXVI (1982), pp. 9-13. (*)

${ }^{437}$ Cfr. M. Stol, Studies in Babylonian History, Leiden (?) 1976, pp. 73-89.

${ }^{438} \mathrm{AHw}, \mathrm{p.} 1171 \mathrm{a}(7)$.

${ }^{439}$ Pour les deux couches cfr. VI.76.20-21.

440I.128.5, 10 (Hanûm); II.53.10, 17 (Hanûm); 79.13 (Ša-pi-El, Hanûm); 98.9' (Hanûm); V.15 (H̆anûm); VII.140+203.2' (Metmiyum [H̆anûm]), 6' (Babuqãn [Ibalahu]), 17' (Zakira-Hăammû [Nahan ou Ibalahuu]); 213.4 (Zakira-Hammû [Nahan ou Ibalahu]); 215.3 (Innahan [Hanûm]), 7 (Zakira-Hammû [Nahan ou Ibalahuu]), 12 (Yapaḥ-Addu [Hanûm]); 227.4' (Yabasu), 7' (Nahanum), 8' (Yabasu), 9' (Yakallit), 11' (Yabasu), 12' (Amurrum), 14' (Nahanum), 16' (Yer'u), 
Dans toute l'étendue du royaume de Mari on trouve des villes dirigées par les sugāgū. Plusieurs de ces villes sont connues par leurs noms. Dans le district de Mari: Hुutnum (XXV.593), qui était une ville ḩanéenne; Amātum et Harratum, habitées par les Yamaḩamméens (IV.1); Sa-ḩușurātim (XXIII, p. 496, n. 113), qui était une ville des Bini-Yaminites, Ḩumzān (XVIII.54.2-3), Huimarān (IX.248.F.8'; XXIV.57), Șubātum (XXIV.60), Sakkā (XXII.326.4-5; XXIV.55) et Tizrah (V.24). Dans le district de Terqa: les lettres mentionnent des localités bini-yaminites dirigées par des sugāgū (II.92.12; III.21.5-7; XXVI.450.6-8). Une de ces villes était Dumtān (III.38.15-17) et une autre Ilum-Muluk (XXIII.237.20). Deux autres villes du district, non habitées par les Bini-Yamina, où on trouve les sugāgū, étaient Hiišamta (VI.40.5-7; XXVI.5.14, 21; 6.54) et Hanna (XXI.198.2-4). Dans le district de Saggarātum on rencontre des sugāgū de villes. ${ }^{442}$ Certaines de ces villes étaient à vrai dire des villages (kaprātum, XIV.8.16-18). On connaît le nom de Dabiš (IX. 248.F.11') habitée par les BiniYamina et Saḩrū (XIV.64.10'-12') habitée par le clan Amnanûm. En outre on trouve les sugāgū à Barḩān (XIV.62), Bīt-Zarḩan (M.11940), Samdanitum (IX.248.F.10'), Utahu (IX.248.F.13'), Yā'il (I.119; M.7407.V), Zarri-Rabbium

17' (Yakallit), 19' (Yakallit), 20' (Ibalaḥu), Rev. 8' (Hanûm); IX.248.15' (Yer'u), Rev.13' (Yabasu), 16' (Yakallit); X.91.3' (Hanûm); XIV.80 (Hanûm); XXI.375.10'-11' (Ȟanûm); XXIII.20 (Hâya-Addu [H̆anûm?, cfr. 225.19]); 222.13 (Layasum [Yabasu]), 16 (Ya'uš-Addu [Yakallit]); XXIV.6.IV.6', 10' (Yakallit), 20' (Hanûm), 25' (Hanûm); 23 (Hanûm); 53 (Yakallit); 56 (Yakallit); 58 ([Yabasu]); 62.3' (Yabasu), 5' (Amurrum), 8' (Nahanum), 16' (Yer'u); XXV.760.6 (Yabasu), Rev.7 (Yakallit), 10 (Ya'uš-Addu [Yakallit]), 12 (Nihadu), 16 (Amurrum); XXVI. 508.8 (Hanûm); A.1691 (Amurrum); A.2741.7-9 (Hanûm); M.7156+ (Amurrum, Ibalahu); M. 8229+ (Amurrum); M.8575+ (Amurrum, Ibalahu, Yer'u); M.11653 (Yer'u); M.11858 (ZakiraHูammû [Ibalahuu], Yer'u); M.12620 (Amurrum); M.12196+ (Zakira-Ḥammû [Ibalahuu]); M. 12283+ (Ibalah̆u, Yer'u); M.12620 (Zakira-Hammû [Ibalahuu]); M.13244 (Hanûm); Ph. Talon, Miscellanea Babylonica, p. 283 (Yakallit). $\left(^{*}\right)$

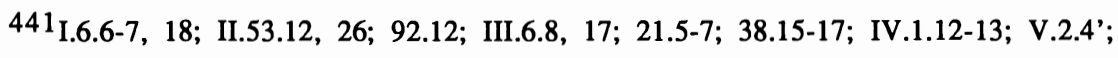
VI.32.16; 38 (cfr. 32 et 38 avec 30); XIV.64.10'-12'; XXIII, p. 496, n. 113; XXVI.24.11; 39.6; 450.7; A.1281. (*)

${ }^{442}$ II.103.12; XIV.65.6; 75.8. 
(XXVI.241.4-6); Zibnatum (XXIV. 60) et Ziniyān (XXVI.462.5-6). Un sugāgum gérait aussi les affaires de la ville fortifiée de Dūr-Yah̆dun-Lim (XIV.46). Enfin un document économique (VII.213.1-9) enregistre les noms de sept hommes du district de Saggarātum. Ces gens étaient tous probablement des sugāgū.443 On fait mention aussi des sugāgū d'Ah-Purattim, la région qui s'étend de Mari à Saggarātum. ${ }^{444}$ Il s'agit sans doute des sugāgū de la population non-tribale. ${ }^{445}$ Pour le district de Qattunān on sait seulement qu'à Tābātum fonctionnait un sugāgum (A.825). Quant au district de Suhum, le district au sud de Mari, on trouve la désignation générale "sugāgum de Suhum"446 et en particulier un sugāgum de la ville de Yabliya. ${ }^{447}$ Le même document que nous avons mentionné à propos du district de Saggarātum (VII.213), enregistre aussi treize noms de gens que nous supposons être des sugāgū. 448 Jusqu'à maintenant nous avons vu que dans chaque district du royaume on trouve des sugāgū à Mari, Terqaa, Saggarātum, Qattunān et Suhum. Mais des sugāgū fonctionnaient aussi dans des régions plus éloignées. Dans la région du Balīh on rencontre le sugāgum de $\mathrm{Dì},{ }^{449}$ qui est à situer probablement dans la région de Ḩarrān. Dans la région d'amont, dans la ville fortifiée d'Abattum, une ville rabbéenne, on trouve aussi un sugāgum (VII.225.9; XXIV. 59). Dans le sud du Djebel Sindjar et dans l'Idamaraş on connaît deux villes où officiaient des sugāgū: Amaz (X.84.5-11), une ville dépendante d'Atamrum, le roi d'Andarig, et Qā (VII.201.Rev'.2'; 140.F.18'). Nous savons aussi que des sugāgū existaient à Razamā-Yamutbal (II.18.7-9). Dans quatre autres villes dont nous ne connaissons pas l'emplacement exact, officiaient des sugāgū: Bīt-huuqqum (VIII.6.12'-13'), Nisiya (XXIV.58.7-9), Samdadû (XXII.153+ 170 [170.3'-4']) et Sakidān (M.15093). Enfin il faut signaler deux villes où fonctionnaient des sugāgū, mais les noms de

\footnotetext{
${ }^{443}$ Iḥid-Irru (1. 3) était le sugăgum de Zibnatum et Hăatni-Addu (1. 15) celui de Samdadû.

444IV.7+M.5737.11, 19; IX.248.F.[14' ?].

${ }^{445}$ Cfr. opposition entre "général des Hanéens" et "général d’Ah̆-Purattim" (M.9649).

446IV.16.11'; XXIV.32.[16]- dix noms, 11. 6-15.

447XXI.333.Rev.68'; XXIII.446.54'.

448Yasìm-Hammû (1. 19) étant le sugăgum de Yabliya.

${ }^{449}$ XXVI.24.16; XIV.114.16, voir XXVI/1, p. 311, note.
} 
ces villes manquent maintenant (car la tablette est endommagée) et il ne reste que le déterminatif KI (IX.248.F.2', 5'). Plusieurs problèmes se posent à propos de la fonction des sugāgū dont nous venons de parler. Pourquoi dans les listes ne mentionne-t-on que les tribus ḩanéennes? Est-ce que les sugāgū qualifiés par le nom d'une tribu remplissaient leur fonction dans le cadre tribal ou étaient-ils à la tête des villages ? Comment faut-il comprendre la différence entre les documents suivants:

A. VII.225.19: [1 mouton-mâl]e Hāya-Addu, Hanéen.

XXIII.20; 21.5-8: 1 habit de serge, ordinaire, 1 arc complexe pour Ḩāya-Addu, le sugāgum homme de Samdadûm.

B. XXI.375.9'-10': 1 habit de serge, ordinaire, 1 arc complexe: Yapah-Addu, le sugāgum hanéen.

XXI.333.67'-68': 1 habit de serge, ordinaire, 1 arc complexe: Yasīm-Hammû, le sugāgum homme de Yabliya.

XXII.326.1-5: 1 habit de serge, ordinaire, 1 arc complexe pour Abdu-Malik, le sugāgum homme de Sakkā.

Dans le groupe A, pourquoi Hāya-Addu est-il qualifié une fois comme Hanéen et une autre fois comme le sugāgum homme de Samdadûm? Et dans le groupe B, pourquoi Yapah-Addu est-il qualifié comme le sugāgum hanéen, tandis que Yasīm-Hammû et Abdu-Malik sont qualifiés comme sugāgū des localités ?

La question est encore plus complexe si l'on prend en considération le fait que beaucoup de Hanéens et de Bini-Yaminites étaient à vrai dire des semi-nomades qui vivaient une partie de l'année dans la steppe et une autre partie dans des villes et des villages.

Dans chaque ville ne fonctionnait généralement qu'un sugāgum, mais on trouve aussi des villes dirigées par plusieurs sugāgū, comme nous l'apprenons par une missive de Yaqqim-Addu, le gouverneur de Saggarātum, qui se plaint à ZimriLim: "Quant au recensement des soldats amnanéens de Saḩrū, j'ai eu beau écrire cinq fois à leurs sugāgū, mais ils ne sont pas venus chez moi" (XIV.64.10'-14'). Peut-être s'agit-il des chefs des quartiers de Sahrū. Une autre lettre qui atteste, peut-être, la présence de plusieurs sugãgū dans une ville - dans le cas présent Saggarātum - était envoyée probablement à Zimri-Lim. D'après cette lettre trois Anciens de Saggarātum vont chez le destinataire, dont le nom manque, pour 
obtenir la fonction de sugāgum (ana sugāgūtim) en apportant avec eux l'argent de leur fonction de sugāgum (VII.311.12-17). ${ }^{450}$ On peut comprendre que les trois se présentent pour la fonction de sugāgum de Saggarătum, mais il se peut aussi qu'ils représentent un quatrième qui va devenir le sugãgum de Saggarātum, à l'instar de deux lettres du temps de Yasmah-Addu, dont nous allons parler par la suite (I.119; V.24). Dans la ville de Sakkā, du district de Mari, deux sugāgū sont attestés pour l'année ZL 2': Abdu-Malik (XXII.326.3-5) et Namišum (XVIII.56.1-4; XXIV.55). De même, nous savons que dans la ville de Samdadû on rencontre le sugāgum Haāya-Addu (XXIII.20; 21.5-9)451 et le sugāgum haanéen Hatni-Addu (XXII.170.3'-4'). Or, nous ne savons pas s'ils ont rempli leur fonction simultanément ou successivement. ${ }^{452}$ Quant aux sugāgū tribaux, les documents de leurs payements nous font connaître cinq sugāgū de Yakallit, ${ }^{453}$ six de Yer'u, ${ }^{454}$ trois de Nahan,,455 un d'Amurrum, ${ }^{456}$ quatre de Yabasu, ${ }^{457}$ et un d'Ibalahuu. 458 Nous ne savons pas quelle était l'importance des unités tribales sous la juridiction de ces sugāgū. En outre, une lettre de Samsi-Addu nous apprend qu'un sugāgum du nom de Ḩatiku du clan Yamaḩammûm était responsable des membres de son clan qui séjournaient dans deux localités différentes (IV.1.5-17). Dans cette lettre il s'agit, nous semble-t-il, des gens d'un contingent en service dans deux villes de Samsi-Addu. On a donc affaire à un séjour temporaire et non pas à un séjour permanent dans ces localités.

Il arrive parfois que les sugāgū soient identifiés par les noms de leurs pères, comme Rip'i-Addu fils ou homme de Ibal-El, le Yer'éen, Milki-Addu fils de Hali-Ḩadun (IX.169), Mutatar, Ani-Lim et Layasum hommes d'Abi-nakar, et

\footnotetext{
450J. Bottéro, RA LII (1958), pp. 164-165.

451 VII.225.19; 226.17 à condition qu'il s'agisse du même personnage.

452 Voir XXIII, p. 28.

453VII.227.F.9', 17', 19'; IX.248.Rev.16'; XXIV.53; 56.

454VII.117.6; 227.F.16'; IX.248.F.15'; M.11357.

455'VII.227.F.6'-7', 14'; M.11357.

456VII.227.F.12'.

457'VII.227.F.4', 8', 11'; IX.70; 248.Rev.13'.

458VII.227.F.20'.
} 
enfin Ibal-pī-El homme de Nisiya[X]. ${ }^{459}$ Dans les deux premiers cas, il pourrait s'agir de pères qui étaient des personnages importants. Ibal-El pourrait être soit un sugāgum lui-même (VII.201.Rev.4') soit le fameux merhum; ${ }^{460}$ Hali-Hadun était, peut-être, un haut fonctionnaire de Zimri-Lim. ${ }^{461}$

Les sugāgū, comme le reste des fonctionnaires d'Etat, étaient considérés comme des serviteurs (wardum) du roi (II.33.9-11; XXVI.450.7; A.1610+.25). Plusieurs lettres nous apprennent la procédure de nomination au poste de sugāgum. La première lettre que nous allons examiner, V.24, a été envoyée par Tarim-Sakim, qui était peut-être le gouverneur de Mari, 462 à Yasmah-Addu: "Baqqum, l'homme de Tizrah (du district de Mari), est allé à son destin, et des hommes, fils de Tizrah, sont venus chez moi en disant: établis Ka'ali'iluma pour nous gouverner, et il a promis une mine $\left(=\frac{1}{2} \mathrm{~kg}\right)$ d'argent pour le Palais. Or çà, maintenant j'envoie Ka'ali'iluma à mon seigneur. Que mon seigneur l'établisse pour la fonction de sugāgum, et qu'on accepte de lui, là-bas, la mine d'argent". On peut supposer que les notables de Tizrah ne se sont pas adressés directement à Yasmah-Addu, parce que celui-ci était absent de Mari. La seconde lettre qui nous concerne est I.119, envoyée par Yasmaḩ-Addu à Samsi-Addu: "Auparavant Yatarum a rempli la fonction de sugāgum de Yā'il (du district de Saggarātum). Et Yatarum est allé à son destin. Maintenant sont venus [chez m]oi cinq hommes ... Yā'iléens ... du hibrum [en disant]: [établis] leur [...] pour nous [gouverner]. Voilà ce qu'ils m'ont dit. J'ai installé [...]-Addu [... à la place de Yata]rum comme sugāgum de [Yā'il]. [...] d'argent [et/ou] 500 moutons [il a donné/donnera au Palai]s [...] moutons [... il est par]ti. Que [Add]â (= Samsi-Addu) sache cela". Relevons les points communs existant entre les deux lettres: les anciens sugāgū sont morts, ce qui peut signifier qu'il s'agit d'une fonction à vie, à la condition qu'elle soit remplie à la satisfaction du Palais. Les notables des villes où les anciens sugāgū ont exercé

${ }^{459} \mathrm{Cfr}$ ci-dessus Chapitre III, $\S 1$.

$460 \mathrm{XVI} / 1$, p. 111 , no. 4 .

${ }^{461} \mathrm{XVI} / 1$, pp. $96-97$.

${ }^{462} \mathrm{Cfr}$. une autre lettre de lui, V.28, qui se termine par la formule typique des gouverneurs: "Tout va bien, Mari va bien, le pays va bien" (11.41-42), et voir aussi V.26.20-21, mais cfr. néanmoins D. Charpin, N.A.B.U. 1989/16. 
leur fonction, vont chez Yasmah-Addu pour présenter leur nouveau candidat. Le nouveau sugāgum ne peut être nommé que par Yasmahn-Addu. Yasmahn-Addu a les pleins pouvoirs, mais il doit rendre compte de l'affaire à son père, "le grand roi". Le candidat promet de payer au Palais dans un cas un mine d'argent et dans le second cas 500 moutons, dont la valeur s'élève à cinq mines $\left(=2 \frac{1}{2} \mathrm{~kg}\right)$ d'argent. Dans la troisième lettre (VII.311) que nous avons déjà mentionnée plus haut, on lit: "Iși-Sarē, Ya'imsi-El, Mut-Ramē, des Anciens de Saggarātum qui sont arrivés [chez moi] pour la fonction de sugāgum. On a contrôlé l'argent de leur fonction de sugāgum. Je remettrai à mon seigneur le montant de [leurs] cadeaux". L'auteur de la lettre semble être le gouverneur de Mari, qui, à la différence de Tarim-Šakim, n'envoie pas le payement pour la fonction de sugāgum au roi par le(s) candidat(s), mais transmet lui-même l'argent au roi. Deux lettres de Bannum, qui datent du début du règne de Zimri-Lim, nous décrivent toute une histoire concernant la nomination d'un sugāgum à Hišamta (XXVI.5; 6). L'important devin Asqudum, qui faisait partie de l'administration d'Išme-Dagān à Ekallātum, obtient de ZimriLim sa nomination comme sugāgum à Hišamta et la nomination d'Enlil-īpuš comme majordome de la ville, et tout cela contre la volonté de Bannum, qui ne voit pas d'un bon oeil la nomination d'un ancien ékallatéen.

Le payement que le candidat à la fonction de sugāgum offre au roi n'est pas un payement unique, mais un engagement de payement annuel, comme il ressort très clairement d'une lettre de Zimri-Addu, le gouverneur de Qattunān, qui écrit à Zimri-Lim: "Auparavant, mon seigneur avait imposé Hammu-tar pour $\frac{2}{3}$ de mine $(=0.33 \mathrm{~kg})$ d'argent quant à la ville de Tāāātum et Hammu-tar a bien payé cette somme à Zakira-Hammû (prédécesseur de Zimri-Addu). L'an dernier, Hammu-tar m'a encore payé cette somme à moi-même. A présent, mon seigneur a désigné Yazḩadum pour la fonction de sugāgum de Ṭābātum. Etant allé à Ṭābātum, j'ai dit à Yazhadum: Les $\frac{2}{3}$ de mine d'argent que Hammu-tar payait à Zakira-Hammû et à moi-même, tu dois les payer" (A.825). La lettre nous montre aussi que le payement représente une somme fixe, que le sugāgum d'une localité devait payer. De plus il est clair qu'à l'époque de Zimri-Lim aussi les sugāgū sont désignés par le roi lui-même et non pas par le gouverneur du district. Le rôle du gouverneur était d'encaisser l'argent et de le transférer à Mari. 
Nous possédons deux groupes de documents où sont enregistrés les payements des sugāgū. Un groupe ${ }^{463}$ comprend de petites tablettes, sur lesquelles sont enregistrées les sommes d'argent ou le nombre d'ovins que le sugāgum devait payer. Le second groupe ${ }^{464}$ comprend des relevés récapitulatifs de payements partiels et des arriérés des sugāgū. Voyons quelques exemples de ces documents: l'arriéré primitif: " $2 \frac{5}{6}$ mines $(=1.40 \mathrm{~kg})$ d'argent de la fonction de sugāgum de Namišum, l'homme de Sakkā, qui n'ont pas encore été reçues" (XXIV.55). Le relevé du premier payement: "Sur $2 \frac{5}{6}$ mines d'argent de Namišum, l'homme de Sakkā, $1 \frac{1}{2}$ mine $(0.75 \mathrm{~kg})$ d'argent a été reçue, $1 \frac{1}{3}$ mine $(0.66 \mathrm{~kg})$ d'argent est son arriéré" (XXIV.61.II.6'-8'; 63.II'.3'-6'). Le relevé des arriérés d'un autre sugāgum: "200 moutons de la fonction de sugāgum de Baḩdi-Addu l'homme de Yabasu (ont été reçus) à Terqa" (IX.70), et le relevé du payement partiel: "1 mine d'argent, 100 moutons de Bahdi-Addu le Yabaséen, 3 ânes, 89 moutons ont été reçus, 41 moutons son arriéré" (IX.248.Rev.13'-15'; XXIV.61.III'.6'-9'). 465 Il se peut que, dans l'une des listes récapitulatives, nous ayons le relevé, non exact, de l'arriéré de Bahdum (= Baḩdi-Addu), qui restait après le payement partiel: " $\frac{1}{3}$ de mine (= $166 \mathrm{gr}$ ) d'argent l'arriéré de Baḩdum le Yabaséen" (VII. 227.F.11'). ${ }^{466}$ Et un dernier exemple, dans le document récapitulatif on lit: "[Sur 2 (?) mines (= $1 \mathrm{~kg}$ ) d'argent de] Yanşibum, [1'homme de Himar]ān [1 mine d'argent a été re]çue, 1 mine d'argent est son arriéré" (IX.248.F.7'-9'). Dans les archives on trouve un document qui affirme la réception du premier payement: "1 mine d'argent de la fonction de sugāgum de Yanşibum, l'homme de Hiimarān. Envoi à la caisse du roi à Mari" (XXIV.57). Un document (M.15093) fait allusion au fait que des moutons livrés par un sugāgum au palais de Mari ont été tondus au palais dans la cour du palmier. On voit donc que le sugāgum versait un payement

\footnotetext{
${ }^{463}$ Par exemple IX.70; XXIV.55; XXV.593.

${ }^{464}$ VII.227; IX.248; XXIV.61-63 (les cinq documents sont des duplicatas); XXV.759-760; M.11858, voir aussi Ph. Talon, RA LXXIII (1979), pp. 149-150 et J. Sasson, M.A.R.I. IV, p. 452.

465 D'après ce document un âne vaut 14 sicles (= 116 gr), $23 \frac{1}{3}$ fois le prix d'un mouton. 46641 moutons valent 24.6 sicles ( $205 \mathrm{gr}$ ) et non pas 20 sicles (166.6 gr), qui représentent $\frac{1}{3} \mathrm{de}$ mine.
} 
au palais pour être nommé, et puis ce payement est devenu une sorte de taxe annuelle. $\mathrm{Ph}$. Talon a fait remarquer que le payement est toujours en argent ou en bétail, jamais en orge, qui était pourtant le payement habituel outre l'argent. ${ }^{467}$ On peut, peut-être, expliquer ce phénomène en le supposant dû au fait que la fonction même de sugāgum a son origine dans la société pastorale. Nous ne savons pas quelle était l'origine de ces payements, on peut seulement suggérer qu'il s'agit des taxes que les sugāgū prélevaient sur la population soumise à leur autorité.

En plus des payements annuels, les sugāgū avaient l'habitude d'envoyer de temps à autre des dons d'ovins au palais, ${ }^{468}$ comme faisaient les vassaux de Zimri-Lim et les autres fonctionnaires de l'Etat.

Deux des trois lettres qui s'occupent de la nomination des nouveaux sugāgū, traitent du district de Saggarātum. Une troisième lettre, de l'éviction d'un sugāgum et de la possibilité de la nomination d'un nouveau sugāgum. Ainsi écrit YaqqimAddu, le gouverneur de Saggarātum, à Zimri-Lim: "Lorsque je me trouvais à Mari, Aham-nūta a parlé en ces termes en présence de mon seigneur: Je ne peux pas continuer à exercer la fonction de sugāgum de Dūr-Yahdun-Lim: je suis chassé. Qu'ils nomment qui ils veulent ! A présent, à cause du rapport que j'ai écrit à mon seigneur, Mašhum, le cinquantenier ${ }^{469}$, est consigné dans la ville, et Ahamnūta est bien à Mari, de sorte que la ville de Dūr-Yahdun-Lim est abandonnée. Maintenant, que mon seigneur exhorte Aham-nūta à partir pour Dūr-Yahdun-Lim. Si Aham-nūta n'y consent pas, que mon seigneur désigne un homme de confiance qui puisse administrer Dür-Yahdun-Lim et qu'il l'envoie (là-bas)" (XIV.46). Cette lettre nous montre que les notables d'une ville peuvent non seulement proposer un candidat, mais ils peuvent aussi, en cas de besoin, l'évincer; 470 cependant l'éviction n'était pas définitive, le roi pouvait soit l'installer de nouveau dans sa fonction, soit nommer un autre à sa place selon son choix. Une allusion à la nomination d'un sugāgum par le roi se trouve dans une lettre d'Inbatum, fille de Zimri-Lim, qui habitait Andarig. Elle raconte à son père qu'un serviteur d'Atam-

\footnotetext{
467 Pouvoirs locaux, p.60.

468VII.140+; 201; 225; 226; XXI.3.5-10; 56; XXIII.222; XXIV.23; 29; 32; A.4586; M.9881.

${ }^{469}$ Pour le titre voir XXIII, pp. 383-384.

${ }^{470}$ Cfr. Ph. Talon, Pouvoirs locaux, p.56, n. 8, qui fait allusion à II.53; 137.
} 
rum, le roi d'Andarig, a reconquis une ville nommée Amaz et y a installé son sugāgum (X.84).

Nous avons vu des cas de sugāgū nommés, peut-être, pour la vie. On peut maintenant ajouter quelques données quant à la durée des carrières de quelques sugăgū: 1. Un sugāgum servait sous deux gouverneurs (A.825). 2. Un autre sugāgum a rempli son poste au moins pendant quatre années (VIII.62.9 [ZL 1']; VII. 106.9 [ZL 4']). 3. Un troisième sugāgum a servi sous Yasmah-Addu à Tizraḩ (V.24) et sous Zimri-Lim à Barhāan (XIV.62).

Nous n'avons que peu d'informations concernant la personnalité des sugāgū. D'après la lettre de Yaqqim-Addu, le gouverneur de Saggarātum, un sugāgum devait être "un homme de confiance" (XIV.46.22), qui pouvait tenir en bon état la ville (Dür-Yahdun-Lim en l'occurrence) qu'il devait administrer (1. 23). Peut-être faisaient-ils partie des Anciens de la ville (Saggarātum en l'occurrence; VII. 311). Comme sugāgum de Hišamta, Zimri-Lim nomma un important personnage - le devin Asqudum (XXVI.5.13-14). Quant à leur statut économique, seul un document (XXIV.6) nous fait savoir que les sugāgū hanéens possédaient des champs (col. IV.3'-25'), comme beaucoup d'autres fonctionnaires d'Etat. De temps à autre le palais distribuait des cadeaux, des vêtements et des arcs aux sugāgū. ${ }^{471}$ Ces cadeaux étaient en vérité une sorte de payement, à l'occasion duquel on organisait un festin pour lequel on faisait sortir du vin des caves royales (IX.101.1-4).472 Comme nous allons le voir par la suite, les sugāgū faisaient aussi partie de l'armée royale, et dans ce cadre ils recevaient des payements comme les autres membres de l'armée. Ces payements consistaient en argent, en vêtements et en objets d'or et d'argent. 473

Le sugāgum était secondé par un majordome (abu bītim) (XXVI.5.13-16), ${ }^{474}$ par un cinquantenier et un conseil des Anciens. ${ }^{475}$ On trouve les Anciens à côté des

471XVIII.54.1-3; 56.1-4; XXI.333.67'-68'; 375.9'-11'; XXII.326.1-5; XXIII.20; 21.5-8; 446.53'-54'; XXV.100.1-3; 623; M.11395.

${ }^{472}$ Cfr. aussi M.11570.

473IV.74; VII.215.

${ }^{474} \mathrm{~A}$ Dür-Yahdun-Lim on trouve un sugãgum (XIV.46) et un majordome (XXVI.6.47).

475 II.103.12-13; XIV.46.6-9, 14; 62.10-12; 64.5-6; 65.6; 75.8; A.2801.35. (*) 
sugāgū dans la société tribale aussi. Les Anciens et les sugāgū ont représenté les Bini-Yamina lors de la conclusion de l'acte d'alliance avec les rois du Zalmaqum dans le temple de Sîn à Harrān (XXVI.24.10-12). Nous devons postuler que les sugāgū ont eu recours à des scribes qui se tenaient peut-être à leur disposition. Ces scribes lisaient sans doute devant eux les lettres qui leur étaient destinées (p. ex. les sugāgū des Bini-Yamina du district de Terqa [III.6.17-18; 38. 15-16] et les sugāgū des Amnanûm de Saḩrū du district de Saggarātum [XIV. 64.12'-13']). Les scribes, placés à côté des sugāgū, écrivaient aussi les lettres de ces derniers, comme la lettre du sugāgum de Dìr (XXVI.24.17). L'administration centrale ne se contentait pas seulement d'écrire aux sugāgū. Quand les circonstances exigaient leur intervention, on les rassemblait dans la capitale du district pour leur donner des ordres, et parfois on devait les menacer pour qu'ils obéissent. Kibri-Dagān, le gouverneur de Terqa, exhorte les sugāgū des Bini-Yamina de son district (II.92.12-20), et Samsi-Addu demande à Yasmah-Addu d'exhorter, lui aussi, les sugāgū des Bini-Yamina de son district (I.6.14-20). De même Yaqqim-Addu, le gouverneur de Saggarātum, rassemble les sugāgū, les cinquanteniers et les Anciens de son district pour leur donner des ordres (XIV. 64; 65). Asqudum, quant à lui, appelle les sugāgū à Raşûm pour qu'ils l'aident à rassembler les Hanéens (XXVI.40.26-27). Ainsi faut-il expliquer, nous semble-t-il, la présence des sugāgū des Hanéens et du district de Saggarātum à Mari (II.98.9'-10'; XIV.80.16-18). Mais parfois les sugāgū refusaient de se rassembler. YaqqimAddu a écrit cinq fois aux sugāgū des Amnanûm qui habitaient Saḩrū concernant un prochain recensement, mais ils ont refusé de venir chez lui (XIV.64.10'-14'). Il arrive aussi qu'on rassemble les sugāgũ de Sahrū, non pas pour les réprimander, mais au contraire, pour les apaiser (IV.16.11'-13').

Les sugāgū se rassemblaient aussi de leur propre initiative pour décider des affaires de la plus haute importance. Une fois il s'agit des sugāgū des Hanéens (A. 2741.9-14), et une autre fois, de ceux des Bini-Yamina (II.53). Quand les sugāgū ont des réclamations à présenter au gouverneur du district dans lequel ils se trouvent, ils s'adressent à lui tous ensemble (II.18.9; XIV.8.5-10).

Une des tâches les plus importantes des sugāgū était de mettre des personnes à la disposition de l'Etat dans le cadre militaire et civil, et cela à la suite du recensement 
qui avait lieu dans leur district. 476 Ils étaient aidés dans cette tâche par les cinquanteniers qui se trouvaient à leur côté (XIV.62.4-12). Parfois on avait recours à des menaces pour obtenir la coopération des sugāgū dans l'exécution de ce devoir (I.6.18-19). Il arrivait aussi qu'on les appréhende tout simplement, et qu'on les tienne prisonniers jusqu'à ce que le quota désiré soit rempli (VI.32.5-16).477 Peut-être, la même raison explique-t-elle le fait que des sugāgū des Bini-Yamina soient retenus chez Zimri-Lim, qui considère la possibilité de les renvoyer dans leurs villes (XXVI.450). Les sugāgũ étaient responsables aussi du recrutement de leurs gens pour des travaux dans les districts: le creusement des canaux et leur nettoyage (III.6), et la moisson des champs royaux (III.38.24-26). Pour effectuer un recensement, probablement celui des Bini-Yamina qui se trouvent dans la steppe, Samsi-Addu ordonne à Yasmah-Addu d'envoyer dans la steppe Lā'ûm et les sugāgū d'Ah-Purattim (IV.7+M.5737). Le rôle des sugāgū dans le cadre militaire ne se limitait pas au recrutement de leurs gens. Accompagnés des cinquanteniers, ils partaient avec les recrues pour les expéditions royales. Ainsi, à côté du commandant Qarrādum, on trouve les sugāgū des Hुanéens qui sont tenus responsables des déserteurs parmi leurs gens (I.128); avec le commandant Ibal-piEl, on trouve des sugāgū au moment de la guerre contre Ešnunna (XXVI.508.78). Qarrādum et les sugāgū formaient même une première instance juridique pour juger un soldat qui avait enfreint le tabou (asakkum) en prenant sur le butin un objet en cuivre (V.72.6'-19'). C'est dans ce contexte que nous comprenons l'une des plus intéressantes lettres d'Asqudum (XXVI. 44). On y lit: "Auparavant, mon seigneur a dit aux sugāgū: Il y a parmi vous une grave impiété (asakkum). On doit 1[aver] cette impiété" (11. 4-6). Ainsi des conjurateurs et des purificateurs vont purifier les Hanéens lors de leur palabre (11. 17-30). Nous pensons que les Ḩanéens ont pris un butin qui leur était interdit, et ainsi ont-ils commis un sacrilège. On trouve aussi les sugāgū à côté du commandant ḩanéen Baḥdi-Addu (XXIII.435), (*) d'Ibal-pī-El (A.1610+.25; A. 1212.24) et du commandant Samidahum, qui leur ordonne d'appréhender les déserteurs et de les amener chez

476II.18.7ss; III.21.5-8; IV.7.11; VI.40; XIV.64.10'-13', et pour 1'"Epopée de Zimri-Lim" voir

D. Charpin et J.-M. Durand, M.A.R.I. IV, p. 327.

477Voir aussi VI.30; 38; XXIII.428; 429 et pp. 358-368. 
lui (I.13.5-30). L'arrestation des fugitifs par les sugāgū ne se limite pas au cadre militaire; dans la vie civile ils sont tenus responsables, avec le garde frontière et les cinquanteniers, pour arrêter des artisans fugitifs (II.103.10-16) ou les fugitifs parmi les gens du Yamḩad et du Zalmaqum qui séjournent dans leur district (XIV.75). Les sugāgũ des villes des Bini-Yamina du district de Terqa avec le garde frontière doivent veiller à ce que leurs gens ne quittent pas leurs villes pour aller dans la région d'amont (II.92.12-22). Ce ne sont pas seulement les BiniYamina qui sont empêchés de transhumer. Les Ḩanéens, auxquels on a interdit de traverser le fleuve pour aller à la steppe, n'ont pas obéi à cet ordre. C'est pourquoi on conseille à Yasmah-Addu que le garde frontière arrête les transgresseurs et les amène à leurs sugāgū qui se trouvent à Mari (II.98.4'-14'). Dans un autre cas les sugāgū et les cinquanteniers devaient assister Itūr-Asdu, le gouverneur de Mari, pour capturer un homme qui avait accompagné un messager d'Ešnunna (A.2801.32-36). La responsabilité des sugāgū envers leurs gens se manifeste aussi par la libération des hommes faits prisonniers par Zimri-Lim (XXIII, p. 497, n. 114). Par contre, nous trouvons un cas qui semble montrer une attitude indigne de la part d'un sugāgum du nom de Mašum qui veut profiter de l'absence d'un de ses hommes qui se trouve en garnison à Ilānșūra pour confisquer sa maison (XXVI.345.21-28).

A une période de disette dans le pays de Mari, Išhi-Addu, le roi de Qatanum, avait invité Yasmah-Addu à envoyer chez lui ses troupeaux. Ces troupeaux furent confiés aux sugāgũ de Zimri-Lim qui avaient été envoyés dans ce but à Qatanum (V.15.12-20). De nos jours, les citadins qui possèdent des troupeaux les confient aux bédouins afin qu'ils les mènent aux pâturages. ${ }^{478}$ C'est aux sugāgū que s'adressent les gens d'Ešnunna avec la question suivante: "Ferai-je passer les moutons par un territoire pacifique ou consoliderai-je mes forces de gendarmerie ?" (XXVI.508.17-20). La responsabilité des sugāgū dans le pâturage se reflète aussi dans un document économique où sont enregistrés 36 ovins qui ont été confiés aux sugāgū dans le district de Saggarātum (XXII.293). On trouve également des sugāgū dans un campement pastoral dans la steppe (IV.7+M.5737.

${ }^{478}$ L. Stein, Die Schammar-Gerba, p. 140. 
21). Enfin, dans une lettre du gouverneur de Saggarātum, Sumḩu-rabi, on parle des ovins du sugāgum de Zarri-Rabbium (XXVI.241.4-6).

Un document de Tell al Rimah nous révèle que les sugāgū étaient responsables aussi envers les autorités centrales pour la fabrication des vêtements par leurs gens (OBTR 100.10-17). On peut ajouter dans ce contexte une lettre de Kibri-Dagān, le gouverneur de Terqa, où est envisagée la possibilité que les villes des BiniYamina fabriquent des vêtements (III.70).

Résumons à présent ce que nous savons à propos des sugāgū. Le mot sugāgum est un mot ouest-sémitique, auquel correspond le mot accadien rabi'ānum. On trouve les sugāgū chez les Hanéens, les Bini-Yamina et la population sédentaire qui n'appartient pas à la société tribale. Nous les trouvons dans toute l'étendue du royaume, dans les districts de Mari, de Terqa, de Saggarātum, de Qattunān et de Suhum, dans les régions du Balīḩ, de l'Euphrate supérieur, en Idamaraș et entre le Djebel Sindjar et le Tigre. On les voit dans le cadre tribal et dans le cadre citadin, ils étaient à la tête de villages et même de vraies villes. Dans les petites localités on trouve un sugāgum et il existe aussi des sugāgū responsables de deux villages. Dans les grandes localités, il y avait probablement plusieurs sugāgū qui exerçaient leurs fonctions de manière simultanée. Dans la gestion des affaires de leurs localités, ils étaient secondés par des cinquanteniers et par un conseil d'Anciens. Les sugāgū pouvaient être proposés au roi pour le poste par les notables des localités ou nommés directement par le roi. Ils étaient considérés comme serviteurs du roi, des gens de confiance. Ils pouvaient rester dans leur poste toute leur vie ou être remplacés si les circonstances l'exigeaient. Le candidat au poste promettait un payement annuel à la caisse royale, et de temps en temps il envoyait des ovins à la capitale du royaume. Le palais, de son côté, octroyait aux sugāgū des cadeaux et des payements. Dans les affaires d'Etat, les sugāgū agissaient tous ensemble. On les rassemblait pour leur donner des ordres, et ils se réunissaient de leur propre initiative pour arriver à une décision commune ou pour s'adresser aux autorités centrales. Ils étaient responsables du recrutement de leurs gens pour l'armée ou pour les travaux civils. Quand ils étaient à l'armée, ils devaient empêcher la désertion de leurs gens. Ils avaient aussi un pouvoir juridique sur ceux-ci. A l'armée ils recevaient une solde du roi en même temps que les autres soldats. Ils devaient appréhender les fugitifs et les déserteurs, et devaient empêcher les mouvements de 
leurs gens interdits par l'Etat. Enfin, ils étaient parfois responsables du pâturage des troupeaux royaux, et de la fabrication des vêtements pour le palais. En fin de compte, les sugāgū représentaient les autorités envers la population, et la population envers les autorités. 479

Comparons à présent le rôle du sugāgum avec celui du moqtar de notre siècle d'une part et avec celui du cheik moderne d'autre part. A propos du moqtar nous lisons:480 "Le Muhtâr est intermédiaire entre le village ou la tribu et les Autorités du pays... il fait fonction de représentant officiel du village ou de la tribu auprès du gouvernement qui lui alloue un traitement mensuel, proportionnel au nombre des habitants du village... Il assiste le gouvernement dans la perception des taxes gouvernementales... il se charge de.l'embauche de la main-d'oeuvre pour l'exécution des travaux publics dans son district... Le Muhtâr est élu par les habitants du village et est nommé officiellement par le gouvernement du district. Déjà lors de l'organisation du cadastre (țâbu) et de l'administration chargée de l'appliquer dans les coins les plus reculés de l'Empire turc, le gouvernement turc eut besoin d'intermédiaires responsables. Et c'est ainsi qu'il nomma des Muhtâr d'abord parmi les fellâhîn et ensuite parmi les fractions des tribus".

A la différence du moqtar, le cheik est élu par sa tribu parmi les familles privilégiées. Il n'a pas besoin d'être nommé par les autorités, et son poste est héréditaire. Son rôle est de veiller à l'intégrité de sa tribu; il assume en temps de guerre et de paix la direction de ses affaires: "Le Šeyh̆ remplit le rôle d'un chef, d'un gouverneur, d'un arbitre et d'un dictateur de la tribu. Le gouvernement turc, au temps de sa décentralisation, n'avait pu maintenir sa domination sur les tribus bédouines par la seule force de son armée. C'est pourquoi l'administration turque a permis aux $\grave{S}$ eyh de se renforcer et d'établir leur propre influence, afin d'obtenir par ce moyen leur soumission à l'autorité du gouverneur turc".481

${ }^{479} \mathrm{H}$. Klengel, Benjaminiten und Hanäer, p. 218.

${ }^{480}$ T. Ashkenazi, Tribus semi-nomades de la Palestine du nord, Paris 1938, pp. 53-54, et voir aussi H. Charles, Tribus moutonnières, p. 19.

${ }^{481}$ Voir T. Ashkenazi, op. cit., pp. 51-53, et voir aussi M.P. von Oppenheim, Die Beduinen, p. 30; H. Charles, Tribus moutonnières, pp. 150-151. 
Il nous semble que l'institution du sugāgum a ses origines dans la société tribale, et qu'avec la sédentarisation de la tribu, l'institution s'est appliquée aussi aux chefs des villages et des villes. Ainsi on peut conclure que le sugāgum englobe le rôle du moqtar et du cheik.

\section{Les Anciens}

Dans les documents de Mari, on trouve plusieurs fois la mention des "Anciens" 482 qui ont rempli leur fonction à côté des rois des villes-royaumes et dans les villes et villages à travers le royaume. Plusieurs lettres nous présentent des Anciens en Idamaraş et dans ses environs en rapport avec des rois. Deux lettres de l'époque de Zimri-Lim traitent d'une ordalie par le dieu du fleuve. L'ordalie doit trancher un litige concernant le droit sur la ville de Sunhum, qui se trouve en Idamaraș. Deux des rois en désaccord, Ili-Addu, le roi de Kiduh, et Ili-Ištar, le roi de Sunā, doivent apparaître au procès avec leurs Anciens, et un troisième roi, Subram, le roi ${ }^{483}$ de Susā, doit apparaître avec les anciens d'Apum (A.1251; XXVI.249). Yawi-Ilā, le roi de Talhāyum, qui se trouve au nord du triangle du Habur, envoie à Zimri-Lim de fidèles Anciens de sa ville (XIII.148. 3-5), et après son assassinat ces sont les Anciens de Talhāyum "qui exercent le pouvoir"484 (A. 2417). Le roi Yarkab-Addu envoie des messagers et des Anciens chez Yawi-Ilā (XIII.145.5-7). Yaqqim-Addu, le gouverneur de Saggarātum, informe Zimri-Lim que Hammu-Labā, le roi d'Ašnakkum, et cinq Anciens de Kurgiš sont arrivés à Saggarātum (XIV.114.5-11). Habdu-Malik, le serviteur de Zimri-Lim, veut conclure une alliance entre Atamrum d'Andarig et Hammurabi de Kurdā, mais il craint que les Anciens du pays de Hammurabi ne fassent pression

482 J.-R. Kupper, Pouvoirs locaux, p. 52; A. Finet, Akkadica XXVI (1982), p. 14. Sur les Anciens à l'époque babylonienne ancienne voir H. Klengel, Or. XXIX (1960), pp. 357-375, H. Rabib, L'institution des Anciens en Israël, Jérusalem 1983, pp. 145-176 (en hébreu) et H. Klengel, "Reflets des Deux Fleuves", Louvain 1989, pp. 63-65.

${ }^{483}$ Ou gouverneur, cfr. D. Charpin, M.A.R.I. V, p. 135 , n. 32.

484J.-M. Durand, RA LXXXII (1988), p. 112. 
sur lui (de sorte) qu'il ne prête pas serment par le dieu (XXVI.393.8-9). ${ }^{485} \mathrm{Et}$ enfin, un représentant de Zimri-Lim s'adresse à Yamruş-El, le roi d'Isqā et Qā'um, et aux Anciens au sujet de sa crainte devant la possibilité qu'ils rompent leur alliance avec Zimri-Lim (II.75).

D'autres lettres nous informent sur la coopération entre les gouverneurs et les Anciens. Kibri-Dagān écrit à Zimri-Lim que lui et les Anciens de Terqa prient pour le bien-être de Zimri-Lim et de ses armées (XIII.117.6'-7'). (*) D'après une lettre de Bahdi-Lim, le gouverneur de Mari, il a l'intention d'aller chez Zimri-Lim avec Yaqqim-Addu, le gouverneur de Saggarātum, et six Anciens (VI.12. 5-10). ${ }^{486}$ Des Anciens remplissent aussi leur fonction à côté du commissaire (hazannum) de la ville bini-yaminite de Samānum, du district de Terqa (III.73.9). Les Anciens d'Urubān et de Dunnum et le gouverneur Kibsi-Addu écrivent une lettre à Zimri-Lim (XXVI.503.3-7). Selon J.-R. Kupper l'institution des Anciens n'existait pas à Mari, la capitale du royaume. ${ }^{487}$ Cela est différent de ce qui est observé à l'époque des anciens rois (voir ci-dessus Chapitre I, § 1). Dans plusieurs lettres, on voit des Anciens d'une certaine localité qui apparaissent ensemble avec un personnage, le seul à être appelé par son nom. Il nous semble que dans la plupart des cas il s'agit du sugāgum de la localité ou de l'unité tribale à laquelle appartiennent les Anciens. Yaqqim-Addu informe Kibri-Dagān qu'Iridum et dix Anciens hananéens vont chez le roi (III.65). Asqudum et Hुali-Hadun, le Hanéen, envoient à Zimri-Lim Niqma-El avec les Anciens de Qā (II.95). Samiya et les Anciens de Subat-Enlil ont écrit une lettre au serviteur de Yasmah-Addu (M.2). Dans les documents sont mentionnés aussi les Anciens de Tizrah̆ du district de Mari (VII.130.1-2), les Anciens de Zabalam du district de Terqa (IX.241.7), les Anciens de Ḩurrān du district de Saggarātum (A.3993.41'), les Anciens de Saggarātum (VII.311; XXVI.206.13, 33), ${ }^{488}$ les Anciens de SubatEnlil (M.2; A.2821), les Anciens de Sunā, Urgiš et Šenah̆ (XXIII.504.4, 6, 9), ceux de Hiwilat et de Talmuš (IV.68.5-8), ceux de Sarimā, à l'est du Tigre

\footnotetext{
${ }^{485} \mathrm{XXVI} / 2$, p. 210 , n. 14.

${ }^{486}$ Ces Anciens sont, sans doute, les Anciens de Saggarātum, cfr. VII.311.12-15.

${ }^{487}$ M.A.R.I. IV, p. 464.

${ }^{488}$ Et aussi, peut-être, VI.12; XIV.121.28.
} 
(SH.861.14-16) et peut-être aussi les Anciens de Kulhitum du district de Terqa (III.83). Les Anciens de Tuttul apparaissent dans une lettre de Yaqqim-Addu sous l'appellation "les Anciens de la ville" (XIV.55.22). Les Anciens d'une ville apparaissent dans une autre lettre, mais dans un contexte qui n'est pas clair (X.162.23). Les Anciens du district, qui apparaissent dans deux lettres à côté des sugāgū et des cinquanteniers, sont les Anciens qui remplissent leur fonction dans les villes du district (XIV.64; 65). Dans une lettre de Yanșib-Addu, on trouve (avant la cassure dans la tablette) les noms de 8 "Anciens du district supérieur" (XXVI.447.7). A la tête d'un relevé, rédigé à Terqa et qui donne la liste de divers militaires et civils, se tiennent 46 Anciens (XXI.388.1). Nous ne savons pas s'il s'agit des habitants de Terqa ou de gens qui sont venus de Mari avec YasmahAddu. 489

A côté des Anciens des villes, on trouve dans une lettre d'Išme-Dagān à son frère Yasmahy-Addu "les Anciens du pays" (IV.29.22), qui semblent être les Anciens des tribus nomades qui séjournent en Idamaraș. La comparaison avec deux autres lettres (II.16; IV.40), qui traitent de la même affaire, nous montre qu'il s'agit très probablement des Anciens de la tribu Hुanûm (IV.40.6, 8). "Les Anciens du pays" à l'est du Tigre apparaissent dans une lettre de Samsi-Addu à Kuwari, le roi ou gouverneur de Sušarrā (SH.878.8). Il pourrait s'agir ici des Anciens du pays de Sušarrā lui-même. On trouve aussi un rassemblement des Anciens du pays, serviteurs de Simaḩlānē, le roi de Kurdā, avec les dirigeants de la tribu Numhāā pour un contrôle dont la nature nous échappe (XXVI.463.5-8). On trouve les "Anciens du pays" aussi au temps de Hुammurabi, le roi de Kurdā (XXVI.393.8, 12). "Les Anciens du pays" d'Atamrum participent au repas royal d'Atamrum (XXVI.438.8').

Nous avons vu des Anciens ḩanéens en Idamaraș, maintenant nous allons examiner d'autres témoignages de l'existence des Anciens dans le cadre tribal. Le premier cas se trouve dans une lettre de Kibri-Dagān d'après laquelle nous comprenons que Zimri-Lim lui a demandé de venir chez lui avec les Anciens de BiniYamina (II.83.11, 18). On ne peut pas décider si nous avons affaire à des Anciens qui remplissent leur fonction dans le cadre tribal ou, au contraire, ce qui semble

${ }^{489}$ Voir XXI, pp. 517-519. 
plus probable, s'il s'agit des Anciens des villes bini-yaminites du district de Terqa. Dans le second cas, il est question d'une alliance qui a été conclue entre Asdi-Takim, le roi de Harrān, et les rois du Zalmaqum d'une part, et les sugāgū et les Anciens des Bini-Yamina, d'autre part (XXVI.24.10-15). Ici aussi on doit choisir entre deux possibilités: les sugāgū et les Anciens habitent-ils dans des villes de la région du Balih ou appartiennent-ils aux tribus bini-yaminites qui séjournaient dans le Zalmaqum ? Peut-être faut-il préférer la seconde possibilité parce qu'elle explique pourquoi les Bini-Yamina ne sont pas représentés par leurs rois, qui régnaient dans la région du Balīḥ, mais par les sugāgū et les Anciens.

Voyons à présent ce que nous savons de l'activité des Anciens: Ils se rassemblent pour une consultation (IV.29.28). Ils doivent se rassembler chez Kibri-Dagān (II.83.19). Yaqqim-Addu, Asqudum et Ašmad les convoquent par des signaux lumineux (XIV.121.28; XXVI.40.25-26; A.3567). Le gouverneur de Saggarātum $^{490}$ réunit les Anciens de la ville devant la porte de la ville pour écouter un prophète de Dagān (XXVI.206). Ils vont en délégation chez le roi à Mari.491 Trois Anciens de Saggarātum vont à Mari afin de payer pour la fonction de sugāgum (VII.311.12-18). Les Anciens de Ḩanûm en Idamaraş ont conclu une alliance avec Išme-Dagān (II.16; IV.40), et les Anciens de Bini-Yamina avec leurs sugāgū ont conclu une alliance avec les rois du Zalmaqum (XXVI.24.11). Atamrum, le roi d'Andarig qui cherche des alliés contre Hammurabi, le roi de Kurdā, s'adresse à Haqba-Hammu et aux Anciens de Numhā pour conclure avec eux une alliance (XXVI.404.35-51). Yawi-Ilā, le roi de Talhāyum, envoie des Anciens avec des instructions pour présenter son affaire à Zimri-Lim (XIII.148. 3-5). Après l'assassinat de Yawi-Ilā par les gens de Luhâ qui ont conquis la ville de Talhāayum après s'y être infiltrés par une brèche, les Anciens de la ville écrivent à Zimri-Lim pour qu'il envoie le nouveau roi (A.2417). Des messagers avec des Anciens de Yarkab-Addu (le roi d'une ville non identifiée) vont chez Yawi-Ilā pour lui exposer la position de leur maître (XIII.145.5-7). Les Anciens de Razamā assiégée sortent de la ville pour négocier avec Atamrum qui assiège la ville et lui

\footnotetext{
${ }^{490} \mathrm{XXVI} / 1$, p. $435, \mathrm{n}$.

${ }^{491}$ II.83.10-12; III.65; VI.12.5-10; XIII.148.3-5; XIV.114 (suivi par III.46); cfr. aussi M.6874. 17'-19'.
} 
proposer un armistice, mais il refuse (XIV.104+.11-12).492 Kibri-Dagān, le gouverneur de Terqa, et les Anciens de la ville prient dans le temple de Dagān pour le salut de Zimri-Lim et de ses armées qui, en collaboration avec les rois de Subartum (Idamaraş), combattent contre Qarni-Lim (XIII.117.6'-7'; (*) III.17.1420). Nous ne savons pas qui étaient les Anciens auxquels Zimri-Lim fait allusion dans une lettre à Buqāqum (XXVI.479.10-14). Et enfin, comme les autres fonctionnaires dans le royaume, ils envoient des ovins au Palais (VII.130. 1-2; XXIII.504.1-9; OBTR 201.5) et même du miel (IX.241.7-8). Dans un document de Tell al Rimah nous lisons qu'un Ancien de la ville de Sušarrā se trouve parmi des gens qui ont reçu de la bière du Palais (OBTR 267.12).

En conclusion, nous voyons que l'institution des Anciens existe dans la société urbaine et dans la société tribale. Ils sont considérés comme les représentants de la communauté à laquelle ils appartiennent, ainsi ils pouvaient la représenter vis-à-vis des autorités, négocier en son nom et établir des accords. 493 Ils représentaient leur communauté dans des cérémonies religieuses. Et enfin, ils participaient au choix des fonctionnaires d'Etat.

Sur le rôle des Anciens dans la société tribale des temps modernes, nous lisons: "Les anciens de la tribu (Ihtyâriyeh) remplissent le rôle de conseillers auprès du Seyḥ et du Muhtâr. Le nom "ancien" (Iḩtyâr) outre qu'il dénote l'âge, est en même temps chez les Bédouins une sorte de titre... Ensemble avec le Šeyh et le Muhtâr, ils veillent au bien-être de la tribu".494 On peut supposer qu'à l'époque de Mari, les Anciens ont rempli dans la société tribale le même rôle.

${ }^{492} \mathrm{Cfr} . \mathrm{XXVI} / 2$, p. 32.

493" Decision-making group " dans la terminologie de J.T. Luke (Pastoralism, pp. 88, 90).

${ }^{494}$ Voir T. Ashkenazi, op. cit., pp. 55. 


\section{Le Hazannum}

Le titre hazannum "maire", "commissaire" apparaît plusieurs fois dans les textes de Mari. ${ }^{495}$ Dans la plupart des cas il s'agit de la région d'Idamaraș. ${ }^{496} \mathrm{La}$ ville d'Amaz est dirigée par un hazannum de Sukru-Tešub, le roi d'Eluhtum (II.109. 9). A la tête de la ville de Talhāāyum se trouvait un hazannum qui était un serviteur de Yahdun-Lim, et à présent le roi de la ville, Yawi-Ilā, un vassal de Zimri-Lim, demande à Zimri-Lim d'envoyer un hazannum dans cette ville (XIII.143.8, 5'). Ḩāya-Sūmû, le roi d'Ilānşurā, adresse une question ironique à sa femme, la fille de Zimri-Lim: "Exerces-tu ici (en Ilānșurā) la charge de ḩazannum ?" (X.32.12'13'). ${ }^{497}$ Et enfin, Zimri-Lim a installé un ḩazannum dans la ville de Šenaḩ dans le cadre d'une campagne militaire en Idamaraș (X.121.7-8). On voit donc que dans tous ces cas il s'agit de villes dépendantes, et le hazannum sert de représentant d'un roi étranger, un résident, ${ }^{498}$ un commissaire. Dans le royaume de Zimri-Lim on trouve un hazannum à Tuttul, du nom de Lanasûm (II.137 passim), et un autre à Samānum secondé par les Anciens (III.73.9). Or, ces deux villes étaient des villes-royaumes bini-yaminites au temps de Yahdun-Lim. D'après les textes du temps de Zimri-Lim, seul Samānum continua d'être bini-yaminite, et c'est peutêtre la raison pour laquelle Zimri-Lim installa là-bas son hazannum. Tuttul était peut-être encore sous l'influence des Bini-Yamina, ce qui obligea Zimri-Lim à y installer son hazannum. On voit donc que le hazannum ne fait pas partie des dirigeants tribaux, mais qu'une population tribale pouvait être gérée par lui.

495 XV, p. 206; CAD H, pp. 163b-165; AHw, pp. 338b-339a, 1560b; J.-R. Kupper, Pouvoirs locaux, pp. 48-50; J.-M. Durand (N.A.B.U. 1987/12c) lit ḩasşi'ânum (XIII.143), hasşsânûtu (X.32) et traduit le premier par "administrateur militaire" et le second par "garnison".

496J.-R. Kupper, Pouvoirs locaux, p. 49.

497Voir J.-M. Durand, M.A.R.I. III, pp. 168-169.

${ }^{498}$ Cfr, A. Finet, Syria XLI (1964), pp. 130-131; Annuaire de l'Institut de Philologie et d'Histoire Orientales et Slaves XX (1968-1972), Bruxelles, 1973, p. 233; Akkadica XXVI (1982), pp. 2-3. 


\section{L'assemblée}

Dans la société tribale, l'assemblée des membres de la tribu, présidée par le cheik et les Anciens, est l'institution dans laquelle sont discutées toutes les question importantes de la vie de la tribu. ${ }^{499}$ Dans les textes de Mari, on ne trouve pour les Bini-Yamina qu'un témoignage de l'existence de cette institution (puhrum). Les Bini-Yamina délibèrent d'un projet de razzia sur les villages des Bini-Sim'āl qui se trouvent dans l'Ah-Purattim (les Bords-de-l'Euphrate). Yaqqim-Addu informe Zimri-Lim qu'il a envoyé deux hommes "à l'assemblée des Bini-Yaminites afin de recueillir leurs intentions" (XIV.84+.4-6). Dans cette lettre, on trouve aussi quelques informations sur la procédure de la délibération. Avant la défaite d'Ešnunna devant Zimri-Lim, l'assemblée avait dressé le plan d'action suivant: "Tandis que le roi est dans son camp, allons razzier l'Ah-Purattim et donnons-lui du tracas !" (11. 12-14). Après la défaite d'Ešnunna, ils réitèrent leur plan: "Maintenant que le roi a remporté la victoire sur Ešnunna, et qu'ils se relâchent, allons razzier et rendons un unique service à Ešnunna" (11. 18-22). Quand ils se préparent à réaliser ce plan, "Un homme se leva dans leur assemblée" (1. 25), et il les informe que les Bini-Sim'āl sont tous entrés dans les places fortes et que leur projet est à abandonner (11. 27-33). A la suite du discours de cet homme, les BiniYamina envoient des espions pour s'informer sur le fond de l'affaire (11. 34-38). Tenant compte du rapport des espions, les Bini-Yamina ont changé leur plan et se sont mis à conquérir la ville d'Abattum, en amont de Tuttul (11. 43-48). De cette lettre on ne peut pas savoir s'il s'agit d'une institution permanente ou si au contraire nous avons affaire à une institution ad hoc. Il faut aussi remarquer qu'on ne parle dans cette lettre ni des rois de Bini-Yamina, ni de leurs sugāgū, ni de leurs Anciens.

Quant aux Ḩanéens, avant qu'ils n'arrivent à des décisions, ils se rassemblaient et menaient des discussions entre eux qui étaient appelées rihsșum "palabre" d'après l'étude de J.-M. Durand. ${ }^{500}$ Les lettres d'Asqudum nous donnent une certaine idée du déroulement de ces palabres. Dans un cas (XXVI.45), Asqudum informe

\footnotetext{
${ }^{499}$ Voir T. Ashkenazi, op. cit., pp. 51, 55; M.P. von Oppenheim, Die Beduinen, p. 30. ${ }^{500} \mathrm{XXVI} / 1$, pp. $181-187$.
} 
Zimri-Lim qu'il a entendu des reproches contre le merḩum Ibal-pi-El, de la part des Hanéens l'accusant de ne pas transmettre les ordres de Zimri-Lim. Surtout on craint un certain Itti-Lim qui risque d'exciter les Hanéens lors du palabre au sujet d'Ibal-pi-El. Pour éviter tout danger, Asqudum décide d'éloigner Itti-Lim et de retenir tous les gens qui se lèveront lors du palabre et parleront contre Ibal-pī-El. Lors d'un de ces palabres, on va purifier les Ḩanéens d'un asakkum: "Lorsque les Hanéens feront le palabre, ils (les conjurateurs et les purificateurs) doivent les purifier" (XXVI.44.29-30). Enfin, c'est à l'occasion de l'assemblée des Huanéens qu'Asqudum leur transmet les instructions de Zimri-Lim (XXVI. 46.5-7).501

${ }^{501} \mathrm{Ph}$. Talon (Miscellanea Babylonica, pp. 278-279) cite la phrase suivante du document $\mathrm{M}$. 11858: (argent) ša LÚ.MEŚ a-bu ka-a-li [X?], ù su-ga-gi, ša Ha-na ša a-di-ni la ma-ah-ru. Cette seule référence ne permet pas d'élucider le sens du terme a-bu ka-a-li. 



\section{Chapitre VI: L'économie des tribus}

\section{Introduction}

On trouve les tribus amurrites de Mari principalement dans deux régions: celle qui est délimitée par l'isohyète de $100 \mathrm{~mm}$ jusqu'à l'isohyète de $200 \mathrm{~mm}$, où on peut pratiquer seulement une culture irriguée, et celle qui est délimitée par l'isohyète de $200 \mathrm{~mm}$ jusqu'à l'isohyète de $400 \mathrm{~mm}$, où l'on peut pratiquer l'agriculture sur des terrains arrosés par la pluie, mais où il est quand même préférable de se servir d'irrigation artificielle (voir ci-dessus Introduction, § 3). Dans ces deux régions, on peut nourrir les troupeaux en hiver en les faisant paître dans la steppe. Pour garantir leur stabilité économique, les tribus ont fondé leur économie sur deux facteurs principaux: l'élevage et l'agriculture. A ces deux facteurs s'ajoutent des branches économiques secondaires: le tissage, la chasse, la razzia, la cueillette et le commerce. Par la suite, nous allons décrire brièvement l'ordre des principales activités économiques des tribus semi-nomades. ${ }^{502}$ Avant la période des pluies, qui commence aux mois d'octobre-décembre, on prépare les champs, et après les premières pluies, on les ensemence. Dans les terres irriguées, on sème d'abord, puis on inonde les champs, ensuite on irrigue cinq ou six fois durant la saison. Dans certaines régions, il suffit d'irriguer les champs trois ou quatre fois. Après l'ensemencement commence la transhumance dans les régions qui sont considérées comme la propriété des tribus (dïrā). Parfois toute la tribu transhume, en lais-

${ }^{502}$ D'après J.-R. Kupper, Les nomades, p. X; J.T. Luke, Pastoralism, pp. 28, 31, 117-118; H. Klengel, $Z$ wischen Zelt und Palast, pp. 27, 52, 172-175; E. Wirth, Agrargeographie des Irak, Hamburg 1962, pp. 24, 62, 64, 101-102, 128; Syrien, Darmstadt 1971, p. 92; J. Weulersse, Paysans de Syrie et du Proche-Orient, Paris 1946, pp. 145-146, 151-153; L. Stein, Die Schammar-Gerba, pp. 32-33, 67-68, 71, 77, 94, 118-119, 121-122, 130; H. Charles, Tribus moutonnières, pp. 23-24; E. Marx, American Anthropologist LXXIX (1977), pp. 345-347; M. Stol, Bulletin of Sumerian Agriculture II (1985), pp. 119-126; N.N. Lewis, Nomads and settlers in Syria and Jordan, 1800-1980, Cambridge 1987, pp. 1-6, 180-187. 
sant derrière elle plusieurs gardiens et responsables de l'irrigation; parfois seulement une partie de la tribu part, et quelquefois seuls les troupeaux et les bergers partent. En hiver et au printemps, la steppe se couvre d'une riche végétation de pâturage, et il existe de nombreux points d'eaux: puits, sources et fossés où l'eau s'accumule. Si le pâturage est riche on peut se contenter d'abreuver les troupeaux une fois tous les quatre jours. Durant cette période les membres de la tribu s'occupent de la fabrication des laitages et des produits manufacturés à partir des produits de leur cueillette et de leur chasse. A la fin de la période des pluies commence la tonte. Aux mois de mars-mai, le soleil ayant desséché la steppe, les troupeaux retournent vers les régions habitées. Les membres des tribus moissonnent le blé dans les champs et font monter les troupeaux sur le chaume. Au mois de mars, on sème le sésame et on le récolte à partir des mois de juin-juillet. ${ }^{503}$ En été, les troupeaux se nourrissent de paille et il faut les abreuver une fois par jour. Entre les mois de février et d'avril on récolte dans la steppe des champignons, on capture des sauterelles et on chasse des biches.

Pour illustrer la transhumance des semi-nomades, nous avons choisi la description de la transhumance des habitants des villages des 'Agêdât à proximité de Tell Harìri, site de l'ancienne ville de Mari. Prenons comme exemple le village Rhabra qui se trouve près de Tell Harīin: les troupeaux partent pour les pâturages au mois de novembre. Ils restent à Bîr Dheïna trois mois, puis ils passent deux mois à Bîr Joubb. Ils retournent pour un mois à Bîr Dheïna et au début de l'été, ils rentrent à Rhabra et paissent le chaume. Les membres des tribus continuent encore à vivre dans les tentes installées dans les champs moissonnés. Il arrive aussi qu'ils s'éloignent dans leur transhumance jusqu'à Alep où ils reçoivent un meilleur prix pour leurs troupeaux. ${ }^{504} \mathrm{Ce}$ voyage dure environ un mois et demi. Les villages près d'Abou Kémâl constituent un autre exemple. Les troupeaux sortent pour le pâturage dans l'oued de Ratqa, où l'on trouve un excellent pâturage. Pendant les années arides, ils vont jusqu'à Bîr Homeïmé qui se trouve sur le plateau de la steppe. Le

${ }^{503}$ D'après M. Stol (Bulletin of Sumerian Agriculture II [1985], p. 119) l'ensemencement normal se fait au mois d'avril-juin et la récolte au mois de septembre-octobre tandis que l'ensemencement hâtif se fait à la mi-mars et la récolte à la mi-juillet.

${ }^{504}$ Voir H. Charles, Tribus moutonnières, p. 116. 
pâturage dans l'oued de Ratqa attire aussi les habitants de la Djéziréh qui traversent l'Euphrate avec leurs troupeaux. .05

Les 'Agêdât caractérisent ainsi leur manière de vie: "La moitié de l'année les 'Agêdât sont pareils au sédentaire et l'autre moitié semblables au bédouin." 506 C'est ainsi qu'on pourrait sans doute également caractériser la manière de vivre des tribus semi-nomades à l'époque de Mari.507

\section{Le pâturage}

Le terme le plus fréquent pour désigner les troupeaux de menu bétail est nawûm, qui comprend les campements et les troupeaux. ${ }^{508}$ On connaît le nawûm des Hanéens, ${ }^{509}$ des Bini-Yamina, ${ }^{510}$ des habitants d'Ah-Purattim (BENJ., p. 988a) ${ }^{511}$, de Yahdun-Lim (AREP, p. 109, 1. 29), de Zimri-Lim (XIV.86.19; A.638+.49; A.1089.16; AREP., p. 109, 1. 25), de Suhum (XXVI.420. 10) et de la ville de Karkamiš (IAMH., p. 49, 11. 4-5). Plusieurs verbes décrivent les diverses activités qui sont en rapport avec le nawûm: Le campement de nawûm est décrit par le verbe sakānum. ${ }^{512}$ Cette racine, sakānum et non pas la racine accadienne

$505_{\text {ibid., p. } 117 .}$

$506_{\text {ibid., p. } 23 .}$

507 Voir J.T. Luke, Pastoralism, pp. 77-78.

508 M.B. Rowton, Or. XLII (1973), pp. 252-253, 256; JESHO XVII (1974), p. 18 définit le nawûm comme "nomadic migratory group", et J.-M. Durand le définit comme: "partie de la communauté qui vit loin de la ville, en déplacement dans la steppe", XXVI/1, p. 342, n. e, voir aussi $C A D$ N1, pp. 249-251.

509II.59.4, 11; III.15.10-11; LANG., p. 109; OBTR 9.13', cfr. aussi l'expression Hanûm ḩibrum ša nawîm "Les Ḩanéens, une unité tribale dans la steppe" (M.5172) et: (Bini-Yamina) hibrum ša nawîm (XXVI.168.20).

510XIV.85.6-7; A.4530-bis. 6'; BENJ., p. 986, n. 1; N.AN., p. 52, no. 6; I.91+.18' (?). 511 IV.7+.10, 13, 16, 21.

512II.35.8, 19(!); XXVI.180.5, 12, 14; 392.39; BENJ., pp. 986d-987a; p. 986, n. 1; AREP., p. 109, 1. 25. Dans LANG., p. 109 est transcrit šaknu, mais il faut collationner le texte pour voir s'il ne s'agit pas d'une faute de frappe et cfr. XXVI/2, p. 225, n. i. (*) 
šakānum, est une racine amurrite, qui est réservée dans la langue de Mari au campement de nawûm. Le pâturage de nawûm est décrit par le verbe akālum, ou par sa forme complète ritam akālum..$^{513}$ Le nawûm descend du plateau steppique au fleuve (warādum, II.90.9), 514 traverse le fleuve (ebërum, III.15. 18) et se rassemble (paḩārum, III.15.27; kamāsum, XIV.121.47; BENJ., p. 989c, 1. 25). Quand tout est en ordre on rapporte que le nawûm va bien (̌̌alāmum, šulmum). 515 Par contre il arrive qu'on razzie le nawûm (šahățum, ${ }^{516}$ mahāṣum, BENJ., p. 992b, 1. 11'). Et on prend des présages pour le salut du nawûm (XXVI.180.6-14; A.3000.38-39). Signifiant au départ "troupeaux de menu bétail", le mot nawûm a fini par désigner les régions où les troupeaux paissent, les régions de pâturage, ${ }^{517}$ et même l'herbe que les moutons mangent (XIV.85.6-8, 11-12). Dans le nawûm, dans le sens géographique du terme, habitent des Hanéens (I.6.26, 41; 42.5, 10), et un hibrum des fils d'Awin (VIII.11.21). On y va ou on y entre ${ }^{518}$, on en revient (II.48.8) et il se rassemble (XXVI.168.20-21). Par la suite nous allons traiter essentiellement des nawû des tribus. Les plus significatives à propos de la composition de la société bini-yaminite sont les paroles des dirigeants de Mišlān qui demandent au roi bini-yaminite Sūmu-dabi: "Il ne faut pas que notre Seigneur montre de la négligence, mais qu'il écrive à ses frères (les autres rois bini-yaminites) afin que soit rassemblée leur troupe qui habite les villes et l'unité tribale dans la steppe" (XXVI.168.17-21). D'abord nous allons essayer d'examiner si l'on peut localiser leurs lieux de pâturage. Dans plusieurs

513II.59.6; 90.10; III.15.13; VI.42.19; XIV.85.8.

${ }^{514}$ Il faut souligner qu'il s'agit d'une restitution [ur-du-nim]-ma, mais la comparaison avec I.83.35; XIII.102.22 montre qu'elle est sûre.

515II.33.21'-22'; 37.25; 59.11-13; AREP., p. 109, 1. 29.

516 BENJ., p. 988a; 991c, 1. 22; OBTR 9. 13'. $\left(^{*}\right)$

517Voir Encyclopaedia Biblica IV, p. 749 (en hébreu). Selon J.T. Luke (Pastoralism, pp. 100101, n. 107) et M. Weippert (Die Landnahme, p. 115, n. 1) le sens original était "région de pâturage" et le sens "troupeaux et bergers" est un sens secondaire. Il faut souligner avec H. Klengel (Benjaminiten und Hanäer, p. 214) qu'assez souvent on ne voit pas clairement lequel des deux sens convient.

518II.45.[10']; 98.7'; IV.7.10; XIV.92.19-20; A.2392. 
lettres, il est question des Hanéens qui se trouvent dans le nawûm. Yasmah-Addu demande à son père: "Les Hanéens du nawûm peuvent-ils prendre (ou) ne pas prendre des champs au bord de l'Euphrate ?" (I.6.26-28). Yarìm-Addu a effectué un recensement parmi les Huanéens du nawûm afin de mettre sur pied un effectif de deux mille hommes qui seront mis à la disposition de Yasmah-Addu (I.42. 57). A l'époque de Zimri-Lim, Bahdi-Lim annonce à Zimri-Lim que les Huanéens sont rentrés du nawûm dans leurs villes (II.48.8-9). Dans deux documents sont enregistrés des vêtements qui étaient distribués aux "Hुanéens qui sont revenus du nawûm" (XXII.147; 171.Rev.4'-11'). Le premier document date du IXème mois, on peut donc conclure qu'ils sont revenus du pâturage hivernal. Dans tous les documents que nous venons de mentionner, on ne dit pas où se trouve le nawûm, par contre on peut localiser les pâturages d'après les lettres de Kibri-Dagān, le gouverneur de Terqa. Kibri-Dagān conseille à Zimri-Lim qu'un nawûm de Hanéens ne paisse pas sur la rive ultérieure de l'Euphrate, mais sur la rive citérieure de crainte de l'ennemi, les Bini-Yamina sans doute (III.15). Peut-être faut-il mettre la lettre que nous venons de mentionner en rapport avec la proposition que Zimri-Lim fait aux Hanéens de rassembler leur(s) nawûm('s) au bord de l'Euphrate car il va infliger une défaite aux Bini-Yamina (BENJ., p. 989c, 11. 24-26). La ville de Terqa, siège de Kibri-Dagān, se trouve sur la rive droite du fleuve. Il s'avère donc que les pâturages des Hanéens étaient sur la rive gauche, dans la Djéziréh, et Kibri-Dagān voulait qu'ils traversent le fleuve pour passer sur la rive droite, dans la Chamieh. ${ }^{519}$ Une autre lettre de Kibri-Dagān nous apprend que, sur la rive droite de l'Euphrate, paissait un nawûm des Bini-Yamina (II.90. 7-12). Enfin, Išhi-Addu, le roi de Qatanum, propose à Yasmah̆-Addu que les moutons de Yasmah-Addu et les Hanéens traversent le fleuve, sans doute l'Euphrate, pour paître dans son pays (V.15). Il s'agit dans le cas présent des Huanéens qui sont chargés des troupeaux de Yasmah-Addu. Dans le même ordre d'idées il est intéressant de constater que le responsable hanéen des pâturages (merhum), Ibal-El, était responsable du nawûm de Zimri-Lim qui paissait en Idamaraṣ. 520

${ }^{519}$ Voir la photo de la traversée de l'Euphrate par des moutons sur des radeaux dans H. Charles, Tribus moutonnières, $\mathrm{Pl}$. V (en face de la p. 48).

520 D. Charpin et J.-M. Durand, RA LXXX (1986), pp. 152-153. 
Nous avons un témoignage d'après lequel les Sutéens paissaient près de l'Euphrate: ils font descendre les troupeaux vers l'Euphrate pour les abreuver à l'époque de la moisson, époque du retour du pâturage hivernal (I.83.34-37). ${ }^{521}$ La présence des Sutéens près de l'Euphrate est également attestée par une lettre du temps de Zimri-Lim. Même s'il n'est pas précisé qu'ils sont avec des troupeaux, on peut tout de même le comprendre du fait qu'on emploie le verbe sakānum: "Les Sutéens campent près de l'Euphrate à une distance de 3 bērū $(=30 \mathrm{~km})$ en amont de Terqa" (III.12.10-11). Des ovins des Sutéens qui paissaient dans Suhum sont attaqués par le gouverneur Buqāqum dans un acte de représailles (XXVI.483. 1011).

Nous avons vu qu'un nawûm d'une tribu pouvait paître sur les deux rives de l'Euphrate, dans la Djéziréh et dans la Chamieh. On peut ajouter un autre témoignage à ce fait. Le haut fonctionnaire de Zimri-Lim, Asqudum, conseille d'installer des gardes sur la rive citérieure d'Appān, du district de Mari, jusqu'à un certain puits pour empêcher probablement les Hanéens d'atteindre le nawûm (II.98. 4'-10'). Où se trouve le nawûm ? Tout dépend du sens à donner au verbe etēqum employé pour décrire le mouvement des membres de la tribu. Si le sens à donner à ce verbe est ici "poursuivre son chemin", "continuer son chemin", les Huanéens veulent aller dans un pâturage qui se trouve dans la Chamieh. Si, par contre, le sens à donner est "traverser un fleuve",522 alors les Hanéens veulent traverser l'Euphrate vers la Djéziréh. Un mouvement en sens opposé, de la Djéziréh à la Chamieh, est décrit dans une lettre de Lā'ûm, le gouverneur de Mari (V.81). Dans cette lettre, des troupeaux de la population sédentaire (muškēnum) et des HanéensYamaḩamméens traversent l'Euphrate du qașum $^{523}$ vers les oueds et ils paissent

521 Voir J.T. Luke, Pastoralism, pp. 116-118.

${ }^{522}$ Comme dans II.113.10.

${ }^{523}$ Voir J.-R. Kupper, Les nomades, p. 85, n. 2 et ARMT V, pp. 132-133; XXVI.440-bis.15 (entre Sippar et Allahad); 481.11 (en aval de Yabliya) cfr. aussi 439.16'; 498.12; 499.14; 500. 23 et XXVI/2, pp. 346, 409, n. 54: La supposition de J.-R. Kupper quant à l'identification de la Djéziréh avec le $q$ /kaşum est confirmée par la lettre XXVI.499.12-14: "Ils passent la nuit à Ida (Hît, sur la rive droite de l'Euphrate); ils traverseront (le fleuve) à Sabaşum et iront le long du fleuve dans le kaşum ". 
dans la région de Dūr-Yasmah̆-Addu et en aval. Il est intéressant de noter qu'aujourd'hui aussi cette région s'appelle Wādiyān "oueds". ${ }^{524}$ Il nous semble que l'appellation "Hanéens des oueds" Hanûm ša naḩallim se réfère aux Hanéens qui font paître leurs troupeaux dans cette région (A.915.11'). ${ }^{525}$ Le qașum désigne la région du pâturage dans la Djéziréh. On emploie aussi l'expression "le pâturage du qașum" (V, p. 132, no. 33.9-10). Dans une lettre de YaqqimAddu la population (muškēnum) refuse d'entrer dans les forteresses en disant "la steppe (qaşum) est remplie de nawûm" (XIV.121.41). Dans la steppe (qaşum) paissaient les tribus Numhă et Yamutbal (VI.42.5, 18-19). Une lettre riche en informations sur le nawûm est écrite par Ibal-pī-El. Même s'il s'agit de troupeaux de Zimri-Lim et non pas de ceux des tribus, elle mérite néanmoins d'être citée: "... comme le nawûm était installé (sakānum), mo[i-même] j'ai fait prendre les présages en quatre fois pour le salut du nawûm. La première fois, je (les) ai fait faire pour le nawûm du qașûm, ... La seconde fois, je (les) ai fait prendre pour le nawûm qui s'est installé à l'Orient (aqdamātum) du Djebel Sindjar et à l'Occident (aḩarātum) ... Tout le temps qu'il y aura du p[âturage pour les ovin]s et les bovins je transférerai ce nawûm ... [En troisième lieu], j'ai fait prendre des présages pour le nawûm qui s'est [installé depuis ... jusqu]'à Gaššum ... En quatrième lieu, j'ai fait prendre les présages pour le salut de Gaššum jusqu'au nawûm de Sargon et jusqu'à Lahumu-[...] ..." (XXVI.180). (*) On constate que le nawûm comprend des ovins et des bovins, et que les troupeaux paissent dans la région du Djebel Sindjar et dans la steppe qui l'entoure.

Une autre région où les tribus avaient coutume de faire paître leurs troupeaux était Lasqum ${ }^{526}$ dans le district de Saggarātum: "et tous les campements (nawûmmeš) des Bini-Yamina se trouvent (saknat) à Lasqum et dans la ville de Manuhatān" (BENJ., p. 986, n. 1). Deux lettres de Yaqqim-Addu, le gouverneur de Saggarātum, nous montrent que cette région de pâturage bini-yaminite a été envahie par les Hanéens. Il écrit: "Dans mon entourage, j'ai appris ceci: Les moutons paissent le pâturage (nawûm) des Bini-Yamina à Lasqum. Un Uprapéen est arrivé et a dit:

\footnotetext{
${ }^{524}$ Voir H. Charles, Tribus moutonnières, p. 18.

${ }^{525}$ Cfr. XXVI.481.9: LÚ-MES na-ha-li.

526Dans le secteur de Halébiyé (cfr. ci-dessus Chapitre IV, §3A).
} 
Les moutons paissent ce pâturage" (XIV.85.5-12), et encore "Les moutons des Hanéens paissent jusqu'à Lasqum. Les parcs à moutons (hașērātum) ont atteint Lasqum" (XIV.81.7-8).527 On voit donc que les Hanéens ont envahi la région des pâturages qui était réservée aux Bini-Yamina, et ils y ont même construit des parcs à moutons (ḩașērātum). Le mot ḩașē/ārātum est un mot amurrite, qui n'existe ni en accadien, 528 ni en hébreu. 529 Une intéressante information nous est fournie par Asqudum, d'après laquelle il a envoyé 20 de ses hommes, chacun vers un autre parc à moutons (hașērum) (XXVI.40.24). ${ }^{530}\left({ }^{*}\right)$ Dans les autres passages où on parle des parcs à moutons, ils sont soit razziés soit sur le point d'être razziés. ${ }^{531}$ On trouve aussi un ḩașārum qui appartient à une ville-royaume, Karanā en l'occurrence (XXVI.427.10). Une comparaison entre deux passages pourrait nous renseigner sur la nature de hașērătum:

A.3200.14'-15': mimma ana immerātišu u ana kuštarātišu tugallalma "Si ... tu causes du tort à leurs (Hanéens) moutons ou à leurs tentes".

A.1086.4-6: aššum ḩașerătim u șēnim ša Hanûm-Bini-Yamina mah̄ạșim

"Au sujet de détruire les parcs à moutons et les ovins des Hanéens Bini-Yamina". On voit donc que les hașērātum se réfèrent probablement aux habitats des nomades moutonniers lors de leur transhumance, c'est-à-dire tentes. C'est ainsi qu'est rempli un vide dans les archives de Mari à propos de "l'un des attributs les plus caractéristiques de la vie nomade",532 à savoir la tente, le kuštarum.

La région des pâturages d'une tribu bédouine s'appelle dīrā. Si une tribu étrangère veut paître dans cette région, elle doit payer annuellement à la famille du cheik l'argent de protection (hüwē) en animaux, en orge ou en argent. La permission de

527 Voir XIV, p. 235 , no. $85.5-8$.

${ }^{528}$ En accadien les mots qui désignent l'enclos sont supūrum et tarbāşum.

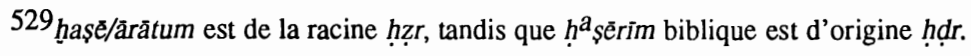

530Pour l'emploi de ÀM cfr. p.ex. XXVI.260.28.

531 II.43.7-8; XXVI.427.10 (suivi du déterminatif KI); A.1086; A.3821.19-20. Et cfr. la razzia du nawûm dans BENJ., p. 992b, 11. 10'-11' avec les razzias de moutons dans XIV.86.21-22. Dans IX.43.5, si la lecture est juste, le sens de ha-sa-[r]a(!?)-ti est comme à l'époque néo-babylonienne "cour", voir CAD H, p. 130b.

532J.-R. Kupper, Nomades, p. 14. 
paître dans le pâturage comprenait aussi l'utilisation des puits. Une tribu étrangère qui pénètre dans ces pâturages sans permission est expulsée par la force. ${ }^{533}$

Lorsque les pâturages manquent à Lasqum, les Bini-Yamina décident de monter en amont de l'Euphrate: "A tous ceux qui demandent, ils répondent en ces termes: Il n'y a plus de pâturage et ainsi nous nous dirigeons vers le Haut-Pays. Voilà ce qu'ils m'ont dit" (II.102.13-17). Yaqqim-Addu, l'auteur de cette lettre, a empêché les Bini-Yamina de monter en amont. Samsi-Addu, quant à lui, traitait les BiniYamina différemment. Il a ordonné à son fils de repousser l'ennemi qui barrait le chemin de transhumance des Bini-Yamina:534 "Dis ceci aux Bini-Yamina: pour n'être plus continuellement coupé des pâturages, je suis monté (ou: je monte) garantir le pays" (I.43.10'-12'). (*) Dans cette lettre il y a, peut-être, une allusion à l'époque de transhumance des nomades. La date de cette lettre est le mois d'Iyar, l'époque de la moisson. On peut supposer que jusqu'à cette date, Sūmu-epuḩ occupait les forteresses frontières au nord de Tuttul, et ainsi durant tout l'hiver il empêchait les tribus de monter aux pâturages dans les régions au nord de Tuttul. D'après une autre lettre, les Uprapéens qui paissent dans les environs de Tuttul vont partir, nous semble-t-il, pour une transhumance qui durera cinq mois (A.4530-bis.4'-18'). La date du pâturage peut aussi être déduite d'une lettre dans laquelle on lit que Asqudum et Hुali-ḩadum vont dans la steppe (nawûm) pour recruter les Hanéens (XXVI.31.5-10). Or, Asqudum dit qu'il "a rassemblé et engrangé le grain du palais" (1. 13), et plus loin on parle "de rassembler rapidement la moisson d'Ah-Purattim" (11. 36-38). Nous sommes donc au printemps, l'époque par excellence du pâturage. Les Bini-Yamina avaient l'habitude de paître les troupeaux aussi au Djebel Bichñ: "Et au sujet des BiniYamina qui ont passé (le fleuve) en direction du Mont Bisir, le roi (Samsi-Addu) m'a écrit (à Tarim-Šakim)" (V.27:25-30).535

Des troupeaux hanéens paissaient aussi en Idamaraş et au sud du Djebel Sindjar. Kabiya, le roi de Kahat, a écrit à Zimri-Lim: "Le campement (nawûm) des Hanéens qui mange (l'herbe) dans mon district va bien. Dans les pâturages, ils

533 L. Stein, Die Schammar-Gerba, p. 130.

${ }^{534}$ Voir J.-R. Kupper, Nomades, p. 63; RLA V/3-4, p. 257a.

535 Voir J.T. Luke, Pastoralism, pp. 77-78. 
reçoivent toute l'eau dont ils ont besoin, et lors du jugement ils obtiennent entière satisfaction. Le campement des Hanéens et la ville de Kahat vont bien" (II.59:413). 536 Dans une phrase qui est citée par Ch. Jean (LANG., p. 109) on lit: "Le campement (nawûm) des Hanéens qui se trouve dans mon district va bien". Il est fort probable qu'il s'agit, ici aussi, d'une lettre de Kabiya. Dans la région de la ville de Kurdā, on a razzié les moutons des Hanéens (XXIII.592.1-4), de même dans une lettre de Tell Rimah on lit: "[...] et Idamaraş se sont joints [...] et ont razzié le campement des Hanéens" (OBTR 9.12'-13'). On peut supposer que les Hanéens paissaient en Idamaraș, et que la razzia a été effectuée par les rois des villes en Idamaraş avec quelqu'un dont le nom n'est pas conservé. * Dans le même contexte, il faut signaler la lettre de Yasìm-El, le représentant de Zimri-Lim à Andarig, qui informe Zimri-Lim que "Maintenant, ces moutons, une troupe idamaraşéenne et numahéenne les a pillés et ils ont emporté ces 1000 moutons et 300 agneaux" (XXVI.422.10-13). Des parcs à moutons des Hanéens se trouvaient aussi dans la steppe entre le Huabur et le Djebel Sindjar (XXVI.40.2224). C'est peut-être vers ces pâturages que les Hanéens installés avec leurs troupeaux (sakānum) à l'est de l'Idamaraş veulent arriver: "Ils font des demandes pressantes pour avoir accès aux pâturages" (XXVI.358.3-7). Une information intéressante et unique est rapportée par Iddiyatum, l'envoyé de Zimri-Lim à Karanā: "Les Numhéens m’ont déclaré ceci en présence de Haqba-Hammû: Vos moutons (mariotes) se nourrissent à nos pâturages et s'abreuvent à nos puits !" (XXVI. 524.35-37). Puis, après une cassure dans la tablette nous lisons: "C'est à la demande de Haqba-Hammû que je viens d'écrire à mon Seigneur. Que mon Seigneur écrive sévèrement aux bergers !" (11. 42-43). Cet incident évoque le passage biblique qui dit: "Les bergers de Guérar entrèrent en contestation avec les bergers d'Isaac en leur disant: Ces eaux sont à nous" (Gen 26:20). Les paroles des Numhéens prouvent que nous avons affaire ici à la dỉra bédouine. A propos des troupeaux d'un homme de Yamutbal, troupeaux qui se trouvent à Andarig, nous apprenons par une lettre de Zakira-Hammu, le gouverneur de Qattunān, adressée à Zimri-Lim, qu'il a reçu une lettre de Qarni-Lim, le roi d'Andarig, disant: "Ibassir [...] et Š̀-pī-El, le Ḩanéen, qui ont razzié les moutons de Ḩāsu-El,

536 Nous pensons que 11. 9-10 se réferrent aussi bien à la 1.7 qu'à la 1.8 . 
le Yamutbaléen, [se trouvent] dans Ašnakkum" (II.79.12-16). La tribu Yamutbal séjournait à côté de la ville d'Andarig (X.84.24-25; 157.15-16). Les Bini-Yamina et les Hanéens paissaient aussi dans la région du Balihh. En effet, dans une lettre de Yaqqim-Addu on lit que, quand Yarīm-Lim (probablement le roi de Halab) assiégeait Ahūna: "les Hanéens, c'est vers les pâturages/campement (nawûm) qu'ils ont fait route (en partant) d'Ahunā" (XIV.92.19-20). Il n'est pas précisé où se trouve le nawûm. Par contre, G. Dossin cite une lettre où il est dit qu'un voyageur qui se rend à Ahunā rencontre sur son passage un campement des BiniYamina en pâturage (saknat, BENJ., pp. 986d-987a). Nous avons quatre détails supplémentaires sur les troupeaux des Bini-Yamina: A. Dans une lettre on lit: "Depuis le jour où mon seigneur a frappé dans le campement des Bini-Yamina" (A.876.9'-11'). B. Un des noms d'années de Yaḩdun-Lim: "Année: Yahdun-Lim est allé à Hên ${ }^{537}$ et a livré à sa main le campement (ou: le pâturage; nawûm) des Bini-Yamina" (N.AN., p. 52, no. 6). C. Zimri-Lim ordonne de détruire les parcs à moutons et les ovins des Hanûm-Bini-Yamina et de faire passer le Balịh (à ceuxci)" (A.1086). D. Les Hanéens vont razzier les moutons des Rabbéens (I.24+M.5012.42-44538). Quant aux Bini-Sim'āl, une lettre nous apprend qu'ils habitaient dans l'Ah-Purattim, et qu'ils possédaient des ovins et des bovins (XIV.84+.27-31).

Les tribus possédaient des troupeaux d'ovins et de bovins ainsi que le prouve le fait que pour obtenir la fonction de sugāgum on payait en ovins, bovins et ânes (voir ci-dessus Chapitre V, $\S 6$ ). Les tribus envoyaient aussi au roi des moutons, ${ }^{539}$ des vaches (VII.227.14') ${ }^{540}$, des boeufs (XXIV.31) et des ânesses (VII.227.10'). Enfin, dans une liste, nous trouvons un habitant de la ville biniyaminite de Dimtān qui était un gardien de vaches (XXIII.86.11-13). Nous avons

537 Voir XVI/1, p. 15 ("Variante de Hana ?") et D. Charpin et J.-M. Durand, M.A.R.I. IV, p. 307, n. 70 ("Cette localité ne saurait être tenue pour variante de Hana").

538 Le numérotage des lignes est d'après la copie, car il y a un décalage entre la copie et la transcription.

539VII.203.1'; 225.13, 19-20; 227.F.13'; XXIII.222.21-31; 341.1-2; 504.10.

540J.T. Luke, Pastoralism, p. 78 suppose qu'ils ont élevé des bovins du fait que dans IV.80.4 il est dit qu'ils ont razzié aussi des bovins. 
deux documents qui attestent les taxes que les tribus devaient payer sur leurs troupeaux: les Sutûm ont livré au palais $2.8 \%$ de leurs ovins comme taxe (miksum; IX.244), (*) et les Ḧanéens ont livré une autre sorte de taxe (laqtum;541 M.11970.58-60). Enfin il est intéressant de noter qu'une espèce d'ânesses était appelée "sutéenne" (I.132.22).

Ibal-El, le responsable des pâturages en Idamaraș, informe Zimri-Lim que "le campement et les Bini-Sim'āl vont bien". ${ }^{442} \mathrm{Il}$ se peut que les troupeaux de ZimriLim étaient confiés aux Bini-Sim'āl pour les faire paître. ${ }^{543}$ Une lettre d'Ibal-pī-El nous apprend qu'il va transférer le nawûm de Zimri-Lim qui paissait près du Djebel Sindjar, et il termine sa lettre par la formule habituelle: "Le nawûm de mon Seigneur et les Bini-Sim'āl vont bien" (A.638+.49). Ces Bini-Sim'āl sont peutêtre les bergers de Zimri-Lim qui sont mentionnés dans une autre lettre qui se réfêre à la même région (XXVI:524.43).

Cinq documents du temps de Zimri-Lim nous donnent une certaine idée sur les dimensions et la composition des troupeaux à l'époque. Le premier enregistre le butin que Zimri-Lim a remporté de cinq villages qu'il a pris: 476 bovins, 34 ânes et 2150 ovins, le second note qu'après sa victoire sur six villages d'Idamaraș, il a ramené 1615 moutons (AREC., p. 104), 544 le troisième enregistre la taxe qu'on a prélevée sur un troupeau sutéen qui comptait 280 moutons (IX.244), le quatrième spécifie que 3360 ovins (des troupeaux royaux) ont été confiés à un berger (XXIII.57.1) et dans un cinquième est enregistré, $d$ 'après une déclaration orale des bergers, un troupeau qui comptait 12441 moutons et chèvres (M.9556).

\section{L'Agriculture}

A. Hanûm - Nous n'avons que peu d'informations sur l'activité agricole des Hanéens. Une correspondance entre Samsi-Addu et son fils Yasmah-Addu traite des champs que les soldats hanéens, qui se trouvaient dans des campements, possédaient dans la vallée de l'Euphrate. Yasmahn-Addu demande à son père: "Les

${ }^{541}$ XXIII, pp. 50-51.

542 Voir ci-dessus Chapitre IV, §6C.

543 Ibidem.

${ }^{544}$ Voir H. Klengel, Zelt und Palast, p. 148. 
Hanéens des campements (nawûm) prendront-ils (ou) ne prendront-ils pas des champs au bord de l'Euphrate ?" (I.6.26-28). Et Samsi-Addu lui répond: "Que ton recensement soit rigoureux ! Et que les Hanéens des campements qui détenaient des champs au bord de l'Euphrate, qu'ils continuent de détenir les champs de la même manière qu'auparavant" (1l. 41-43). Dans une autre lettre qui concerne la même affaire nous lisons que "le recensement va avoir lieu; les hommes seront recensés et les champs mesurés et on partagera de nouveau les champs aux gens du pays" (I.7.32-36). Les Hanéens qui se trouvaient dans les campements, ${ }^{545}$ avaient servi dans l'armée de Yasmah-Addu, et en échange de leur service le roi leur avait offert des champs. L'octroi des champs aux nomades par les autorités centrales sert aussi à les lier à la terre dans le but de les tenir plus fortement sous le pouvoir central. ${ }^{546}$ Dans un relevé cadastral qui note l'octroi de champs aux gens de métier, on trouve les noms de 21 sugāgū des Ḩanéens, qui ont reçu chacun entre $1 \mathrm{IKU}\left(=3600 \mathrm{~m}^{2}\right)$ et $10 \mathrm{IKU}$ et demi $\left(=378000 \mathrm{~m}^{2}\right)$ de champs (XXIV.6.IV.3'-25'). Les champs distribués sont donnés sans doute en guise de payement pour le travail des gens énumérés. Ailleurs, dans un relevé des quantités d'orge appartenant aux localités des districts de Saggarātum et de Mari, nous trouvons la localité hanéenne de Nihad (XXIII.69.10) et la localité de Yamahammûm (1. 9). Les Hyanéens ont pris part aux travaux agricoles dans les champs du Palais. Ce renseignement provient d'une lettre du gouverneur de Saggarātum, Yaqqim-Addu, où il cite une plainte de Zimri-Lim qui pensait que "les Huanéens sont retenus dans les villages à cause du travail des boeufs" (XIV. 80.5-6). Le travail dont il est question pouvait être soit le labour ${ }^{547}$ soit la récol-

545D'après H. Klengel (Zelt und Palast, p. 173) l'appellation "Les Hanéens des campements" sert à les distinguer de ceux d'entre les Hanéens qui s'occupaient de l'agriculture, mais il pouvait très bien s'agir des Hanéens en transhumance.

$546_{\mathrm{H}}$. Klengel, Benjaminiten und Hanäer, p. 215. Pour des exemples des temps modernes voir J. Cernik, Euphrat und Tigris, Petermann's "Geographische Mittheilungen", Ergănzungsheft no. 44, 1875, p. 15; E. Sachau, Reise, Leipzig 1883, p. 264; M.P. von Oppenheim, Die Beduinen, p. 73; E. Wirth, Agrargeographie des Irak, Hamburg 1962, p. 423.

${ }^{547}$ Cfr. III.33. 
te. ${ }^{548}$ Les Yamahamméens possédaient des champs dans le district de Saggarātum, et La'ûm conseille à Yasmah-Addu d'octroyer à un certain individu un des champs des Yamaḩamméens (V.86).

B. Bini-Yamina - Le témoignage le plus significatif sur l'activité agricole des Bini-Yamina se trouve dans les lettres des serviteurs de Yagih-Addu, le roi de Mišlān. Dans ces lettres, il est fait mention de la récolte (XXVI.168.16; 170. 3'14 ') et des champs que possède la ville: "Notre Seigneur le sait: la campagne des terres sillonnées ainsi que la région des puits de Mišlān est vaste. C'est jusqu'à la porte d'Appān (que) les terres sillonnées de Mišlān (s'étendent)" (XXVI.171.1618). Et en effet, une fois ni le palais ni les particuliers n'ayant plus de grain, on conseille à Zimri-Lim de prendre dans Mišlān mille (mesures) de grain (XXVI.181). Une lettre de Yasīm-Sūmu, le chef comptable du palais de Mari, traite des champs dans le pays (erșetum) des Bini-Yamina. L'intendant s'oppose à l'idée de Zimri-Lim d'affranchir les champs du pays des Bini-Yamina, car il a déjà semé le sésame dans les champs des Bini-Yamina (XIII.39). ${ }^{549}$ Nous pensons qu'il s'agit ici des champs qui autrefois appartenaient aux Bini-Yamina qui sont maintenant la propriété du palais ${ }^{550}$ et que Zimri-Lim voudrait restituer aux BiniYamina. Dans une lettre, le roi de Yarihûm, Yasmah-Addu, écrit à Zimri-Lim à propos de l'affranchissement des champs et de quatre villes des Yarihéens, qui étaient affranchies par Zimri-Lim (II.55.26-32). Les habitants de Mišlān se plaignent devant Zimri-Lim d'Asqudum l'accusant de leur avoir pris leurs champs (XXVI.74.5-8). Dans la même lettre, on parle aussi du champ de la ville de Zarri (1. 19). A propos d'un autre roi des Bini-Yamina, Dādi-ḩadun, le roi de Rabbûm, il est dit qu'il fait moissonner son orge à Abattum (XIV.83.36-37). J.-R. Kupper ${ }^{551}$ trouve un témoignage de l'activité agricole des Uprapéens dans les paroles de Lanasûm, le préfet de Tuttul: "Des Uprapéens sont arrivés chez les Tuttuléens. Ils ont permis d'affranchir les eaux du Balīh, et ils ont dit ceci:

\section{${ }^{548}$ Cfr. $A b B$ VIII.98.35-42.}

549 Notre lettre ressemble au document M.8795.

$550_{\mathrm{H}}$. Klengel (Zelt und Palast, p. 176) comprend tout à fait différemment la lettre.

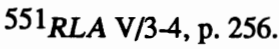


Puisque Zimri-Lim n'affranchit pas pour vous les eaux, envoyez-nous un rapport complet, et alors nous vous livrerons complètement les eaux du Balīh (dont vous avez besoin)" (A.2769.5-15). H. Klengel 552 fait remarquer que même aujourd'hui les paysans et les semi-nomades irriguent leurs champs au moyen des canaux du Balīh. Des attestations supplémentaires sur l'agriculture des Bini-Yamina se trouvent dans des fragments de lettres cités par G. Dossin. Dans une lettre, on lit: "J'entends parler du passage des Uprapéens chez les Rabbéens; jusqu'à présent, ils n'ont pas passé (le fleuve). L'orge des Bini-Yamina est dispersée; ils sont installés sur leurs aires mêmes. Leur rassemblement n'a pas (encore) eu lieu" (BENJ., p. 985a). Dans une autre lettre, on trouve: "Rassemblez (les Hanéens) vos campements (ou: votre campement) sur les bords de l'Euphrate. Je vais infliger une défaite aux Bini-Yamina; après quoi, mangez le blé des Bini-Yamina. Voilà ce que leur a dit mon seigneur (Zimri-Lim), mais ils n'ont pas accepté" (BENJ., p. 989c). G. Dossin écrit aussi "Un fragment de tablette administrative signale la superficie des terres cultivées par les Bini-Yamina et le montant de la redevance à laquelle ils étaient soumis" (BENJ., p. 985b). Dans des relevés où sont enregistrées les taxes sur l'orge des districts de Mari, Terqa et Saggarātum, on trouve les localités des Bini-Yamina: Amnanû (XXIII.69.5), Dabišān (1. 8), Zarri (1. 17; XXIII.426.4), Samānum (XXIII.69.19), Mišlān (XXIII.70.10; 426.3) et Ša-ḩuṣurātim ou: Ša-işurātim (XXIII.70. 11; 426.7). A l'époque de la moisson, les Bini-Yamina devaient prendre part à la moisson du blé du Palais, mais le gouverneur de Terqa, Kibri-Dagān, se plaint auprès de Zimri-Lim: "Et de même, au temps de la moisson, personne ne m'a tiré d'embarras parmi les villes des BiniYamina" (III.38.24-26). ${ }^{553}$ La lettre nous montre aussi qu'à l'époque de la moisson, les Bini-Yamina séjournaient dans leurs villages, probablement venaient-ils à ce moment là d'arriver de leur transhumance hivernale. Dans le cadre du chapitre sur l'agriculture des Bini-Yamina, il faut peut-être citer le fait que des prisonniers bini-yaminites ont été rachetés des mains des paysans (XXII.262.I.29-31, 44-46). La fraction tribale Bīt-Awin a octroyé à l'un de ses

\footnotetext{
552 Zelt und Palast, p. 171.

${ }^{553}$ Voir J.T. Luke, Pastoralism, p. 77.
} 


\section{Les tribus amurrites d Mari}

membres, Yarīm-Addu, un champ de 5400 ares, probablement dans la région d'Appān du district de Mari (VIII.11).

C. Bini-Sim'āl - Dans une lettre prophétique, on lit, d'après la restitution de J.M. Durand: "[afin que fassent] la moisson Zimri-Lim et les Bini-Sim'āl" (XXVI.200.11-13). On voit, donc, que les Bini-Sim'āl s'occupaient d'agriculture.

D. Numḩāa - Avant de conclure une alliance avec les Anciens de Numḩā, Atamrum leur demande s'ils ont quelque chose à lui réclamer. "Ils ont réclamé un champ cultivé" (XXVI.404.41). Cela nous prouve que les Numhéens de la région de Karanā s'occupaient d'agriculture.

E. Résumé - Nous constatons que les Hanéens, les Bini-Yamina et les Sutéens étaient des bergers. Les deux premiers s'occupaient aussi d'agriculture, une occupation qui était étrangère aux Sutéens. Quand on compare ce que l'on sait du travail agricole des Hanéens avec celui des Bini-Yamina, on a l'impression que les Bini-Yamina s'occupaient plus d'agriculture que les Hanéens.

\section{Diverses activités}

a) Le tissage: Le travail du tissage est une occupation typique des tribus moutonnières. ${ }^{554}$ Les Bini-Yamina fournissaient des vêtements au palais par l'intermédiaire du gouverneur et sous sa responsabilité, comme on l'apprend d'une lettre de Kibri-Dagān à Zimri-Lim: "Au sujet des trois cents vêtements la part de mon district . Or çà, les rois des Bini-Yamina se trouvent tous auprès de mon seigneur. Si ces villes doivent faire les vêtements ou bien ne doivent pas (les) faire, ceci ou cela que mon seigneur m'écrive pour que [j'exécute] les ordres de mon seigneur." (III.70+.23-30). En outre, dans une liste, nous trouvons 38 vêtements qui ont été préparés par cinq villes du district de Saggarātum et transmis aux fonctionnaires de l'Etat. Parmi ces villes se trouve la ville de Zarri-Rabbiyum (XXII.121.3-4).

${ }^{554}$ H. Klengel, Benjaminiten und Hanäer, p. 216; Zelt und Palast, p. 185; M.P. von Oppenheim, Die Beduinen, p. 31. 
b) La chasse: Les nomades de tout temps pratiquent la chasse. L'époque de Mari ne fait pas exception. Un Ḩanéen qui a tué un lion a reçu du roi cinq sicles $(=41.6$ gr) d'argent (XXV.143), et un autre Ḩanéen qui a tué également un lion est envoyé au roi (XXVI.106.21-24). Dans une lettre de Yaqqim-Addu, gouverneur de Saggarātum, nous lisons que des Uprapéens dépouillaient un cerf (XIV.86.9-10). A titre de curiosité on peut citer $\mathrm{E}$. Sachau,, 55 qui raconte que la région près du confluent du Habur dans les environs du Bseira (donc dans l'ancien district de Saggarātum) est une région où l'on trouve des biches, et il a même rencontré làbas un chasseur de biches.

c) La razzia: La razzia est une activité économique non négligeable chez les nomades, voir les exemples cités Chapitre IV, § 6Ai; Chapitre VII, § 1c; § 2c; § 4b, c.

d) La récolte des champignons: A l'époque de la transhumance hivernale, les nomades récoltent des champignons. ${ }^{556}$ Cette activité est documentée dans une lettre qui raconte que Șūra-ḩammu, le roi des Amnanûm, a envoyé une couffe de champignons à Zimri-Lim (II.104), à l'instar de Yaqqim-Addu qui lui aussi a envoyé des champignons (XIV.35; 36.16-21).557

e) Le commerce: J.-R. Kupper pense que les 3000 ânes des Hanéens qui reviennent à vide, sans recevoir d'orge et de laine et qui sont mentionnés dans une lettre (B.590.8), montrent qu'ils se livraient au commerce caravanier sur une grande échelle. ${ }^{558}$ Mais il pourrait s'agir aussi de don d'orge et de laine pour leur propres besoins. Les Bini-Sim'āl ont envoyé au palais de Mari huit jarres de vin (IX.15. 6-7). D'après $M$. Birot ce vin leur est parvenu grâce à leur engagement dans le commerce par caravane. ${ }^{559}$ Mais il faut aussi prendre en considération le fait que le pays montagneux au nord de l'Idamaraș, la région par excellence des Bini-Sim'āl, est un pays riche en vignes. ${ }^{560}$ Il est donc possible que des membres de la

555 Reise in Syrien und Mesopotamien, Leipzig 1883, p. 287.

556 Voir les photos des tas de champignons cueillis par les nomades chez L. Stein, Die Schammar-Gerba, photos nos. 29 et 30; H. Klengel, Zelt und Palast, une photo en face de la page 161. ${ }^{557}$ Cfr. aussi D. Charpin, N.A.B.U. $1989 / 58$.

558 Nomades, p. 15.

${ }^{559}$ IX, p. 272, c'est aussi l'opinion de H. Klengel, Zelt und Palast, p. 186.

$560_{\mathrm{E}}$. Sachau, Reise in Syrien und Mesopotamien, pp. 408, 419, 421-422, 444. 
tribu Bini-Sim'āl aient été viticulteurs. Dans un autre document, on note la taxe levée sur un chargement de pierres meulières et de jarres de vin qui se trouvaient dans des bateaux de Ḩammi-ištamar, le roi des Uprapéens (XIII.86.10-12). Dans ce cas, il pouvait très bien s'agir du commerce pratiqué par H̦ammi-ištamar. Il faut aussi signaler que des marchands se trouvent dans la ville bini-yaminite d'IlumMuluk du district de Terqa (XXIII.237.16-18).

f) Varia: Dans une liste qui énumère peut-être des Hanéens, ${ }^{561}$ on trouve un forgeron et un héraut (VII.215.9-10, 35). Des habitants de villes bini-yaminites du district de Terqa étaient des devins (XXIII.593; XXVI.168-172; M.9921. III), et des prophètes nabû se trouvaient parmi les Hanéens (XXVI.216.7), enfin des devins bini-sim'ālites se trouvaient auprès de Zimri-Lim (XXVI.5.46-48).

${ }^{561} \mathrm{D}$ 'après le fait que Innahan, Zakira-Hăammû et Yăpah-Addu $(11.3,7,12)$ étaient des sugãgũ hanéens. 


\section{Chapitre VII: Les relations entre les tribus et les autorités}

Dans ce chapitre, nous devons diviser la discussion selon les différentes époques de l'histoire de Mari: l'époque de Yahdun-Lim, l'époque de Yasmah-Addu Samsi-Addu, Yasmahn-Addu - Išme-Dagān et l'époque de Zimri-Lim, car les relations des tribus avec les autorités sont différentes pendant les diverses périodes. Nous distinguerons parfois entre les diverses régions du royaume, et nous étudierons séparément les diverses tribus, car il existe des différences dans leurs relations avec les autorités.

\section{Hanûm}

A. L'époque de Yah̆dun-Lim - Les inscriptions de Yaḩdun-Lim (IAHD. 15-23; M.2802.III.28-IV.4) racontent comment il a battu les rois des Hुanéens, ou pères des Ḩanéens, qui exerçaient leur autorité sur une région du moyen Euphrate, et comment il a détruit leur centre tribal, la ville de Hुaman. Depuis lors, il était appelé le roi du "pays des Ḩanéens". 562 Il se peut que cette victoire de YaḩdunLim marque le début de la soumission des tribus hanéennes à l'autorité de Mari. En Idamaraş, en revanche, Yahdun-Lim était forcé de payer aux pères des Hanéens l'argent de protection, pour qu'ils permettent à ses troupeaux d'y paître tranquillement (AREP., p. 109). Les documents de Mari nous apprennent, en outre, qu'un individu de la tribu Niḩad a reçu dans le palais de Mari 20 pièces d'arme zamrātum (XXII.224.Rev.5-6), et que deux autres individus, cette fois des clans Naḩan et Yamaḩammûm, ont envoyé des ovins au Palais (XXII.290. 1-3, 68).

B. L'époque de Yasmah-Addu - Samsi-Addu - Cette époque est riche en informations sur les relations entre les Hanéens et les autorités. A cette époque, les

${ }^{562}$ D. Charpin et J.-M. Durand, RA LXXX, pp. 141, 143, 150. 
territoires où vivaient les Hanéens étaient compris dans le royaume même. Les localités ḩanéennes dans la vallée de l'Euphrate étaient soumises à la surveillance de Yasmah-Addu, et l'Idamaraș, où vivaient nombre de H̦anéens, était le coeur même du royaume de Samsi-Addu, dont la capitale était Subat-Enlil.

La grande partie des renseignements sur les Hanéens à cette époque concerne l'engagement des Hanéens dans l'armée de l'Etat. L'armée de Mari était composée des membres des tribus et des habitants des localités non-tribales, "l'armée d'AhPurattim". Avant l'expédition du Zalmaqum, Samsi-Addu écrit une lettre à Yasmahy-Addu qui fait valoir la différence entre les Ḩanéens et l'armée d'Ah-Purattim: "Recense donc les Hhanéens promptement ... Que l'armée d'Ah-Purattim se rassemble à Saggarātum" (II.1.24-25, 29-30). ${ }^{563}$ Avant les expéditions militaires, on recensait les Hanéens. Le recensement était un acte administratif qui se déroulait périodiquement, aussi bien à l'époque de Yasmahn-Addu qu'à l'époque de ZimriLim. Deux termes sont employés pour décrire le recensement. L'un, tēbibtum, qui signifie littéralement "purification", était lié, d'une façon ou d'une autre, à quelques activités cultuelles. ${ }^{564}$ L'autre terme est piqittum, dérivé de la racine paqādum. Les recensements se déroulaient parmi les sédentaires et parmi les seminomades. Le but du recensement était de dresser des listes pour le service dans l'armée ou la corvée, et aussi pour la perception des taxes et la distribution des terres. Les recensements étaient instaurés par le roi et sous la responsabilité des gouverneurs des districts, qui avaient recours aux sugāgū pour les aider dans l'exécution du recensement. Des recenseurs (mubbibum) étaient nommés dans chaque ville. Ils recensaient les gens et les enregistraient d'après les localités où ils vivaient et d'après leurs professions. Puis, les listes étaient envoyées au roi qui les gardait dans une chambre scellée par son sceau personnel. Les gens des tribus qui ne vivaient pas dans les villes étaient rassemblés à un certain endroit et là-bas on les recensait. On trouve des recensements nationaux et régionaux, et on pouvait même recenser des unités déjà existantes. Des documents rendent compte de recensements "purification" (tēbibtum) seulement à l'époque de Yasmah̆-Addu,

${ }^{563}$ Voir aussi la "stèle de Victoire de Zimri-Lim", 11. 9'-10' (D. Charpin et J.-M. Durand, M.A.R.I. IV, p. 320).

${ }^{564}$ Voir J.-R. Kupper, Studia Mariana, pp. 99-110. 
tandis que le recensement (piqittum) des Hanéens est rapporté par des documents de l'époque de Yasmah-Addu et de l'époque de Zimri-Lim.

Nous possédons une série de lettres et de documents économiques qui attestent les recensements des Hanéens avant et après des expéditions. En vue de l'expédition de Yasmaḩ-Addu à Qatanum, Samsi-Addu annonce à Yasmaḩ-Addu qu'il a reçu une lettre du commandant Yarïm-Addu l'informant: "J'ai recensé les Hanéens de campement et j'ai fixé à 2000 hommes (le nombre de ceux) qui devront aller en expédition avec Yasmah-Addu. Et tous ces hommes, nommément, sont inscrits sur tablette" (I.42.5-9). Par la suite, il informe Yasmah-Addu qu'il doit recruter plusieurs milliers d'hommes parmi les habitants de son district et parmi eux un millier de Hanéens (11. 28-29). Dans un autre cas, Yasmah-Addu doit rassembler des Hanéens et les envoyer sous le commandement de Zimri-Addu à Qatanum pour aider son roi, Išhii-Addu (II.5.5-14). Avant l'expédition du Zalmaqum, Samsi-Addu écrit une lettre à Yasmah-Addu qui nous fournit un détail intéressant sur le recensement: "Tu m'as écrit que tu étais actif dans le recensement et que tu n'as pas eu des égards et je m'en suis grandement réjoui. Dès que, (au) pays de Mari, tu en auras fini avec le recensement ... rends-toi à Qattunān, et, là-bas, recense le district de Qattunān et les Hुanéens; et au cours de ton recensement tu ne dois pas avoir des égards envers eux" (I.82.5-17). Les recenseurs enregistrent les gens sur des tablettes qu'ils envoient à Subat-Enlil pour que Samsi-Addu puisse les entendre lire (11. 18-25 et I.87). Deux lettres précisent qu'on doit rassembler les Hanéens pour le recensement dans un endroit spécial. Dans la première lettre, il s'agit de Haššum-de-Membida où les Hanéens ont coutume de se rassembler (I.37.32-41), et dans la seconde lettre, l'endroit est soit Gaššum ${ }^{565}$ soit Sur'um "où se trouve de l'eau" (II.1.24-30). Un recensement d'une grande envergure a été effectué par le chef de l'armée de Yasmah-Addu, Išar-Lim, dans l'Idamaraș supérieur: dans le district de Nahuur, Talhāyum, Kirdaḩat et Ašnakkum. A la suite de ce recensement, Išar-Lim envoie à son maître les listes qu'il a dressées au cours du recensement (V.51). Il est très probable que ce recensement est celui qui est mentionné dans les documents économiques de Chagar Bazar (voir ci-dessus

${ }^{565}$ D'après XXVI.180.26, 29 la ville se trouve dans les environs du Djebel Sindjar et d'après A.1188.Rev. 2' peut-être en Idamaras. $\left({ }^{*}\right)$ 
Chapitre II, § 3, "L’année Adad-bani"). Dans ces documents sont inscrites la nourriture et la boisson qui étaient fournies lors du recensement.

Des Hanéens servaient dans la garde du palais de Samsi-Addu à Subat-Enlil. En effet, Samsi-Addu demande à Yasmah-Addu: "D’entre les Hanéens que tu recenses, prends 400 hommes bons (pour le service) pour se tenir à la porte de mon palais. Parmi ces hommes, que 200 - une section - soient des fils de bonne famille, et que 200 - une s[ection] - soient de solides gaillards pauvres dans une situation économique critique. Les solides gaillards pauvres, dans le palais, je pourvoirai généreusement à leur entretien. Et les fils de bonne famille seront complètement entretenus dans les maisons de leurs pères (bītāt abišunu)" (II.1.10-23). Nous savons aussi que des membres du clan Yamahammûm qui habitaient dans le district de Mari étaient envoyés chez Samsi-Addu pour servir sous son ordre (IV. 1). Enfin, un document (XXV.602) ${ }^{566}$ et une lettre (I.19+M.9541.2'-5') font allusion à l'équipement que les soldats hanéens recevaient.

En dépit du fait que les Hanéens s'engageaient volontairement dans l'armée de Yasmah̆-Addu, on trouve aussi parmi eux des déserteurs (I.6.38).

Les rapports entre le pouvoir central et les Hanéens se maintenaient par l'intermédiaire des sugāgū, qui étaient nommés par l'Etat. Mais il faut souligner que, pour cette époque, nous n'avons qu'une attestation des sugāgū hanéens, et deux autres attestations douteuses. Dans les trois documents en question (I.13; 128; V.72), il s'agit des sugāgū qui servaient à l'armée avec leurs gens et aidaient les commandants des troupes à surveiller les membres des tribus qui se trouvaient à l'armée.

Les bonnes relations qui existaient entre les Yamaḩamméens et les autorités se manifestent par le fait que Lā'ûm, le vizir de Yasmaḩ-Addu, voulait octroyer à un gardien des forêts un champ parmi les champs de Yamahammûm dans le district de Saggarātum (V.86), et aussi peut-être par le fait que des troupeaux des Yamaḩamméens paissaient avec les troupeaux des muškēnum (V.81.5-12).

C. L'époque de Yasmah̆-Addu - Išme-Dagān - Les Hanéens de 1'Idamaraș ont profité du changement de pouvoir et, avant l'arrivée d'Išme-Dagān à Su-

${ }^{566}$ Cfr. le "côté": "(la section de $\mathrm{NP}_{1}$, la section de $\mathrm{NP}_{2}$ et la section de $\mathrm{NP}_{3}$ ) du corps d'armée de Sūmu-Nihum" avec II.5.5-14. 
bat-Enlil, ils ont osé razzier les troupeaux du roi et des notables (IV.80). Après avoir été battus par Išme-Dagān, les Hanéens lui ont proposé de signer un pacte de paix. Et en effet, une alliance fut conclue entre Išme-Dagān et les Hanéens, qui ont, à nouveau, servi le pouvoir central (IV.29; II.16; IV.40). Les Hanéens qui servaient sous Išme-Dagān recevaient de lui des approvisionnements (I. 134).

D. L'époque de Zimri-Lim - Zimri-Lim, comme son père Yahdun-Lim, portait lui aussi le titre de "roi du pays des Hanéens", et il était considéré tout d'abord comme roi des Hanéens et en second lieu roi des Accadiens (VI.76.20-21) ou encore comme "le premier des Hanéens". 567 Il semble que Yahdun-Lim et ZimriLim se considéraient comme les héritiers des rois des Hanéens qui régnaient sur le moyen Euphrate avant d'être vaincus par Yahdun-Lim. Les Hanéens, comme les autres sujets de l'Etat, étaient considérés comme des serviteurs de Zimri-Lim (II. 25.11'). Il est possible aussi qu'ils aidèrent Zimri-Lim dans sa guerre contre Yasmah-Addu, à la suite de laquelle il s'empara de Mari. ${ }^{568}$ Dès son accession au trône, Zimri-Lim est monté au triangle du Habur et parmi ses diverses activités on relève qu'il a offert des cadeaux aux femmes hanéennes et cela pour gagner leurs faveurs (XXII.258). Les bonnes relations entre Zimri-Lim et les Hanéens se voient, en outre, par le fait qu'ils prient pour le salut de Zimri-Lim (A.1191.6-7).

Les Hanéens constituaient la majeure partie de l'armée aussi à l'époque de ZimriLim. Un rapport de Bahdi-Lim, le gouverneur de Mari, nous donne une idée sur la proportion entre le nombre des soldats hanéens à l'armée et le nombre des soldats citadins: "J'ai r[ecensé] parmi les Hanéens quatre m[ille], parmi les habitants de M[ari] et parmi les habitants de T[erqa], cent cinquante hommes et Yasarum, le H[anéen], j'ai placé à leur tête et je l'ai dépêché" (VI.68.6'-12'). Les habitants des villes constituent dans ce cas seulement $4 \%$ de la force recrutée par Bahdi-Lim. Une autre lettre, dont G. Dossin donne deux fragments, nous décrit elle aussi la composition de l'armée: "et avant que les soldats d'Ešnunna ne pillent l'AhPurattim, que le roi avec les Hanéens et toutes ses troupes arrivent à l'Ah-Purattim

${ }^{567}$ D. Charpin et J.-M. Durand, M.A.R.I. IV, p. 328, n. $159,1.3$.

${ }^{568}$ D. Charpin et J.-M. Durand, M.A.R.I. IV, p. 320, 1. 10'. 
pour qu'il n'y ait pas de faute" (AREP., p. 122a, 11.41-44).569 "Toutes ses troupes" sont les sédentaires. Asqudum distingue lui aussi entre les Hanéens qu'il entend rassembler et l'armée qui, en l'occurrence, est déjà prête (XXVI.27.10). D'autres informations à ce sujet sont fournies par la correspondance du commandant Ibal-pī-El, qui s'occupe du retour de l'armée de Zimri-Lim envoyée pour aider Hammurabi, le roi de Babylone, ${ }^{570}$ dans sa guerre contre Rïm-Sîn, le roi de Larsa (XXVI.386), pendant l'année ZL 9': "[Au sujet de] l'envoi de 500 homme d'Ah-Purattim ... [Maintenant] je vais joindre à mon armée les (hommes) des Hanéens et (les hommes) d'Ah-Purattim" (II.25.4, 13-14). (*) Et dans la lettre d'Ibal-El, il parle d'une unité composée de "Cent Hanéens et cent de mes (de Zimri-Lim) serviteurs d'Ah-Purattim" (A.2730.8-9). La même distinction (si les restitutions sont bonnes) se trouve dans deux autres lettres d'Ibal-pi-El qui écrit dans l'une d'elle à propos de la même affaire: "[10 mi $] 1 l e^{571}$ de tes hommes et mille hommes des Hanéens et [d'Ah-Purattim]" (II.23.16), d'après l'autre on envoie une unité de 300 hommes qui est composée de 150 [Hanéens], 50 Suhéens et 100 hommes d'Ah-Purattim" (II.22.20-21). Dans une autre lettre, envoyée par Asqudum et Hali-ḩadum, on trouve aussi la même distinction (XXVI.31.8-9). Cette distinction est confirmée enfin par une lettre qui concerne elle aussi l'expédition à Babylone. ${ }^{572}$ Dans la lettre on trouve une distinction dans l'armée entre "le général d'Ah-Purattim" et "le général des Hanéens" (M.9649). Dans les garnisons qui stationnaient dans les forteresses du Subartum (un autre nom pour l'Idamaraș), on trouve des Hanéens et des citadins (S.115, no. 72-5). Les prisonniers des Hanéens et des habitants de Mari qui se trouvent chez Zaziya le Turukkéen appartenaient, peut-être, à ces garnisons (S.115, no. 72-38). Les Hanéens qui partaient en expédition recevaient, comme toute l'armée, des approvisionnements (XXVI.27.37-39; 29.12').

\footnotetext{
${ }^{569}$ Il est possible que Yaqqim-Addu se réfère à ces Hanéens, lorsqu'il écrit: "quand les Hanéens sont allés faire campagne contre Ešnunna" (XIV.82.19-20).

570Il s'agissait d'une "bonne armée" (XXVI.449.39), et voir aussi XXVI.471.

${ }^{571}$ Cfr. II.25.4.

${ }^{572}$ B. Lafont, N.A.B.U. 1988/29.
} 
Comme nous l'avons vu, avant le départ pour une expédition, on recensait les Hanéens. Le commandant Yantin-Erah raconte à Bahdi-Lim: "Je vais recenser les troupes et ensuite je reviendrai (ou: je vais recenser de nouveau). Si les Ḩanéens, section par section, et clan (gā'um) par c[lan ...] cette armée pour l'ex[pédition] est une armée d'élite, non des réservistes" (VI.28.6-10). (*) Dans des lettres qui datent du 12-13.V.ZL 3,, 573 Asqudum décrit comment il a rassemblé les Huanéens en vue d'une expédition probablement en Idamaraş (XXVI.27; 29.4-5; 30.5-7). Il a envoyé le Ḩanéen Ibal-El chez les Ḩanéens qui étaient dirigés par Hali-ḩadun, leur fixant un terme de six jours pour se rassembler afin d'aller avec Asqudum à Qattunān. $\left({ }^{*}\right)$ Le rassemblement des Hुanéens de Hुali-ḩadum avait lieu dans Saphum, au nord du Djebel Sindjar (XXVI.30.8-9) (74 $^{57}$ et une autre fois à Mahanum (A.1191.4). Dans une autre lettre de Ḩali-ḩadun et Asqudum, on rassemble les Hुanéens et on leur fixe un terme de trois jours (M.11505.4-8), et puis encore, Asqudum annonce à Zimri-Lim que les Hanéens qu'ils veut rassembler viendront dans un mois, mais il est sûr que "Tous les Ḩanéens se rassembleront comme un (seul) homme" (XXVI.40.28, 64).575 Et Zimri-Lim ordonne à Ašmad, du clan Amurrum, de rassembler les Hुanéens au moyen de signaux lumineux pour qu'ils viennent au secours comme un seul homme (A.3567). Hali-hadun lui aussi rassemble les Hanéens par le moyen des signaux lumineux. En effet, il écrit à Zimri-Lim: "j'ai immédiatement allumé trois feux et selon le message de mon maître j'ai rassemblé les Ḩanéens" (SIGN., p. 183, n. 2, 11. 6-9). De même, Asqudum informe Zimri-Lim qu'il a allumé le feu, et que par la suite les sugāgū sont venus chez lui (XXVI.40.25-26). Mais les Ḩanéens ne répondent pas toujours aux appels des autorités. Ainsi Bahdi-Lim, le gouverneur de Mari, a attendu en vain cinq jours pour leur rassemblement (II.48). Les Hanéens étaient considérés, comme nous venons de le voir, comme une "troupe d'élite" (VI.28.89). La "troupe d'élite" qui est allée avec Zimri-Lim à Ugarit (A .2966+3053.1618) était, pensons-nous, composée de Ḩanéens (voir ci-dessus Chapitre II, § 3 ,

\footnotetext{
${ }^{573}$ Voir XXVI, p. 142.

${ }^{574} \mathrm{XXVI} / 1$, p. 163 , n. a.

${ }^{575}$ Le rassemblement des Hạnéens est mentionné aussi dans une autre lettre d'Asqudum (XXVI. 43).
} 
"L'année ZL 8' "). Ulluri, l'envoyé de Zimri-Lim, déclare à propos de l'armée de Zimri-Lim qui va monter en Idamaraș: "Ne sais-tu pas que la lance de Zimri-Lim et des Hanéens est plus forte que celle du pays tout entier ?" (XXVI.303.31'-33'). Et Zimri-Lim lui-même fait l'éloge des Hanéens dans une lettre à Hammurabi: "Parmi les troupes hanéennes que [je t'ai envoyées] choisis 200 ou 300 hommes, qu'ils s'installent [...] face à l'ennemi, et ainsi l'ennemi [ne pourra pas faire] quoi que ce soit. Et les troupes, autant que je t'en ai envoyées, tu [...], ces troupes, comme une armée de 30000 hommes, pourront enlever les obsta[cles devant toi]" (II.67.8'-13'). ${ }^{576}$ Hammurabi était si content de l'armée que lui avait envoyée Zimri-Lim qu'il ne s'était pas hâté de la lui renvoyer, en dépit du fait que ZimriLim en avait besoin. ${ }^{577}$ Le commandant Ibal-pī-El, qui était responsable de cette force, écrit à Zimri-Lim plusieurs lettres concernant le renvoi de cette armée à Mari. ${ }^{578}$ A propos des Hanéens qui ont pris part à la conquête de Larsa nous lisons: "Alors que, déjà auparavant, j'avais vu l'ardeur des Hanéens, en aucune occasion je ne leur avais vu une telle ardeur" (XXVI.386.3'-5'). Une lettre envoyée à Zimri-Lim depuis la Babylonie est unique en cela qu'elle nous fait connaître le nom d'un clan hanéen qui s'était mis aux ordres de Hammurabi; il s'agit du clan Yakallit: "Yakallit [s'est disp]osé à se mettre au service [de Huamm]urabi" (XXVI.377.19-22). Il est intéressant de signaler que ces Hanéens avaient été mêlés, lors de leur séjour en Babylonie, à une affaire qui risquait d'entraîner l'ordalie par le fleuve. Sîn-bēl-aplim, le préfet du palais de Hammurabi, déclare: "Il faut que les Hanéens qui ont saisi le jeune serviteur viennent plonger dans le fleuve" (XXVI.257.10'-11'). A propos du moral qui régnait dans les unités hanéennes, nous lisons dans une lettre du commandant Bahdi-Addu: "(Quand) je prêtais l'oreille à toutes les expéditions (antérieures), il y avait beaucoup de plaintes. Maintenant, je prête l'oreille à cette expédition, il n'y a ni plainte, ni quoi que ce soit (de semblable). Il n'y a que rire et plaisir, comme s'ils étaient chez eux, leur coeur est satisfait. Le coeur des serviteurs de mon seigneur médite sur l'effort dans la guerre et sur la défaite de l'ennemi. Que mon seigneur soit

${ }^{576}$ Voir J.T. Luke, Pastoralism, p. 169, n. 18.

${ }^{577}$ Voir XXVI/2, p. 195 , n. e.

${ }^{578}$ Cfr. XXVI/2, pp. 148,195 , n. e, pp. $376-377$. 
joyeux" (II.118.10-23). ${ }^{579} \mathrm{Par}$ contre, Ibal-pī-El craignait une éventuelle rébellion dans l'une de ses unités, probablement une unité hanéenne (II.25.12'-13').

Des soldats hanáéns ont été amenés d'Idamaraş à Terqa par Kibri-Dagān. 580 Trois lettres racontent les étapes de leur voyage. La première lettre nous informe l'arrivée à Qattunān de mille soldats, qui forment l'avant-garde. Ils seront suivis par deux mille Hanéens (III.14). La seconde lettre raconte une étape nocturne à mi-chemin entre Qattunān et Saggarātum (XIII.102). La troisième lettre de la série raconte l'arrivée de la force à destination, à Terqa: "Trois mille [hommes], autant que mon seigneur m'a écrit, vont tous se rassembler" (XIII.103.29-31). Mais il arrive aussi que les Hanéens refusent d'aller à l'Aḥ-Purattim: "Les Hanéens m'ont répondu ceci: Nous n'irons pas sur l'Ah-Purattim. C'est au milieu de l'Idamaraş que nous habitons. Il n'y a personne qui veuille aller sur l'Ah-Purattim" (M.6148).

La qualité des soldats hanéens se manifeste aussi par le fait que, parfois, un petit nombre de soldats suffisait pour mener à bien des opérations militaires. Il suffit de cinquante hommes de Hanûm pour convaincre l'Idamaraș de soutenir Zimri-Lim (XIV, p. 239, no. 106.19'); deux cents Hanéens étaient envoyés par Hali-ḩadun pour libérer Kurdā du siège d'Ešnunna (A.2821) et pour défendre le district de Saggarātum, Zimri-Lim fournissait au gouverneur seulement cent Hanéens (XIV.121.5-8). Par contre, les 20 Hanéens qui étaient envoyés par Ibal-pi-El n'ont pas sauvé Talhāyum (A.1610+.47-48; A.1212.45-48). Des Hanéens escortaient aussi des caravanes. En effet, deux Hanéens escortaient un émissaire de Zimri-Lim qui conduisait une caravane de Mari en direction de Elahut (dans le Zalmaqum) et qui fut attaquée à mi-chemin (II.123).

Les Hanéens en garnison dans les villes de Mari et de Șuprum recevaient des rations d'huile pour leur entretien. ${ }^{581}$ A la tête de ces Hanéens se tenait Ka'alaEl. ${ }^{582}$ A propos de cette garde hanéenne de Mari, nous sommes informés par une lettre de Hali-hadun et Ka'ala-El ("nos gardes vont bien" [XXVI.106.7-8]). Ils

\footnotetext{
${ }^{579}$ Cfr. aussi XXVI.386 et XXVI/2, p. 205, n. b.

${ }^{580}$ Voir J.-R. Kupper, Syria XIL (1964), p. 107.

${ }^{581}$ TEM III, pp. 16-19, AB; XXII.40; 41.

582Voir XVI/1, p. 134; XXIII, p. 613b.
} 
ont débarrassé la ville d'un danger causé par deux lions qui ont fait leur gîte au soir à la porte de la ville (XXVI.106). On trouve Hुali-ḩadun aussi à la tête de petits groupes de Hanéens qui apparaissent dans des listes de personnes qui ont prêté serment par le dieu. ${ }^{583}$ Les Hanéens qui étaient, sans doute, en garnison à Qattunān recevaient leur entretien du gouverneur. S'ils constataient qu'on les avait privés de la quantité d'orge qu'ils pensaient leur être due, ils se levaient et quittaient le district (B.63.33-48). Une autre lettre fait état, elle aussi, des Hanéens qui se trouvent dans le district de Qattunān (XXVI.141). Zimri-Lim ordonne au gouverneur du district Zikri-Addu de rassasier en orge les Hanéens qui souffrent de la faim, afin qu'ils gardent le district contre la menace d'un ennemi dont le nom n'est pas donné. Et le devin Nūr-Addu prend des présages pour le salut des ces Hanéens (XXVI.141.17-18). Une allusion aux Hanéens qui se tenaient auprès du roi se trouve dans une phrase écrite par un fonctionnaire de Zimri-Lim: "Ne saistu pas que la parole de Zimri-Lim (parvient au) souverain (d'Elam) ? Je demandais: Comment ? Il a répondu: Le Hanéen qui se tient auprès du roi révèle (la chose) à Išar-Lim" (II.124.8-14). Ce sont, sans doute, ces même Huanéens du service de Ka'ala-El dont Zimri-Lim écrit à Asqudum: "Tous les réquisitionnés, pour aller à Abullātum, ont abandonné leur garde et sont partis" (XXVI.52.6-9). Cet acte est considéré par Asqudum comme une très grave offense, une transgression du tabou royal (11. 9-10). Les unités hanéennes ont été commandées par les chefs de l'armée ("les grands des Amurrites"). L'un d'eux, Baḩdi-Addu, était lui-même un Hुanéen qui remplissait aussi la fonction de sugāgum. ${ }^{584}$ Comme nous l'avons vu ci-dessus, c'était lui qui informait Zimri-Lim sur le moral des troupes hanéennes (II.118). Bahdi-Addu commandait aussi l'unité hanéenne qui avait été renvoyée par Ibal-pī-El de Babylonie: "Bahdi-Addu [a pris] la tête de cette armée, et je les ai envoyés à mon seigneur" (II.25.7'-8'). Bahdi-Addu informait le chef comptable du palais de Mari, Yasīm-Sūmû, de l'avancement de cette armée dans son chemin vers Mari: "Les troupes passent la nuit à Dīr. C'est (demain) matin seulement qu'elles atteindront Mari. [Or], les hommes ont faim: ils

${ }^{583}$ XXIII.85.44; 235.IV.5-6.

${ }^{584}$ Voir M. Birot, Syria XII (1964), p. 52. Il est possible que le commandant Yasarum appartenait lui aussi aux Ḩanéens (VI.68.10'), mais la restitution n'est pas sûre (XVI/1, p. 14). 
n'ont pas reçu de vivres" (XIII.33.8-14).585 C'est sans doute à propos de cette même armée hanéenne que nous lisons dans une lettre du devin Erib-Sîn que 50 Hanéens qui ont fait partie de l'armée parvenue à Hanat (= Âna), ont déserté sous prétexte qu'ils n'ont pas reçu leur approvisionnement (XXVI.97.7'-11'). Un certain Yakūn-Arari leur promet de l'orge, mais ils n'acceptent pas et partent (ll. 12'-20'). Dans une lettre de Bahdi-Lim, le gouverneur de Mari, on trouve plusieurs détails sur les commandants et l'équipement de l'armée: "... qu'il mange le repas. Demain matin, qu'on prenne pour les centeniers et les cinquanteniers toutes les ceintures (?) (ou: équipements [?]) dont j'ai parlé à mon seigneur, et qu'on apporte aux Hanéens toutes les armes zamrātum disponibles. Les Hanéens n'[ont] pas de flèches flambantes (?). Autre affaire. Si mon seigneur veut donner un siège au commandant des troupes, qu'on m'apporte un siège" (VI.69.2'-12'). Nous possédons aussi un document où sont enregistrés les soldes en objets d'or et d'argent et les vêtements qui ont été donnés à Bahdi-Addu, à Yantin-Erah et à leurs hommes (XXIII.435). ${ }^{586} \mathrm{D}$ 'après ce document, nous constatons que l'unité de Bahdi-Addu comptait 1500 soldats, 15 centeniers et 30 cinquanteniers, 3 sugāgū et un scribe. L'unité de Yantin-Eraḩ comptait, quant à elle, seulement 500 soldats, 5 centeniers, 10 cinquanteniers, 50 dizeniers, un devin et un scribe..$^{587}$ (*) Après le recensement d'une armée en vue d'une expédition, on nommait son chef: "nos alliés babyloniens, eux, établissent un chef d'armée ("grand-des-Amurrites") pour deux (ou) trois cents hommes ... A présent, des troupes nombreuses vont partir et leur chef n'est pas désigné. Que Yantin-Eraḩ conduise les troupes à bon port et en outre qu'il rejoigne les troupes et qu'il prenne les troupes en charge; que Zimri-Addu soit le chef d'armée, et que Yantin-Erah soit son lieutenant" (VI.28.13-25). Dans une autre lettre, ce même Yantin-Eraḩ est nommé par ZimriLim comme chef d'armée des Hanéens (M.9649.4"'-5').

A l'époque de Zimri-Lim comme à l'époque de Yasmahn-Addu, les sugāgū contrôlaient les Huanéens. Les sugāgū avaient, à l'égard de Zimri-Lim, la responsabilité de faire sortir les gens de leurs villes du district de Saggarātum, probablement

585 Voir M. Birot, note précédente.

${ }^{586}$ Cfr. IV.74.17-39.

${ }^{587}$ Et voir XXIII, pp. 381-385. 
pour des corvées (XIV.80), et pour le service dans son armée (II.33). ${ }^{588}$ Les sugāgū joignaient leurs efforts à ceux de l'Etat afin d'empêcher les Hanéens de passer de leurs villes du district de Mari à la steppe (II.98). C'est le second cas où l'on constate qu'il y a une certaine tension entre les Hanéens et Zimri-Lim (le premier cas est II.48), mais il faut faire attention au fait qu'il y a une tension entre plusieurs Hanéens et leurs dirigeants qui se trouvent à Mari, et devant lesquels on doit amener les transgresseurs. Des Hanéens du district de Saggarātum ont envahi les pâturages des Bini-Yamina, contre le gré du gouverneur (XIV.81. 4-16; 85); Zimri-Lim a profité une fois des services d'un sugāgum hanéen pour obtenir une information délicate (X.91.3'-7'). Les sugāgū adressent aussi à Zimri-Lim des demandes concernant les relations de l'Etat et d'Ešnunna (A.2741).

Par deux fois, Zimri-Lim a voulu octroyer aux Hanéens un village ou de l'orge qui appartenaient aux Bini-Yamina (BENJ., p. 984d, e; 989c). Ces actes font partie de la politique de Zimri-Lim envers les Hanéens. A l'aide de l'approvisionnement, il voulait les calmer. Nous possédons plusieurs témoignages de cette pratique en Idamaraş. Ibal-El, le merhum d'Idamaraş, informait Zimri-Lim qu'il avait établi la paix entre les Hanéens et l'Idamaraş, et à la suite de cette alliance, les Hanéens étaient rassasiés et ainsi ils ne voulaient plus faire la guerre avec l'Idamaraș (II.37). ${ }^{589}$ A la suite de cette alliance entre les Hanéens et les rois d'Idamaraș, ceux-ci fournissaient, sans doute, des approvisionnements aux Hanéens. Cette politique est exprimée explicitement par Zimri-Lim dans une lettre de sa fille Kirûm, la femme de Ḥāya-Sūmû, le roi d'Ilānșurā (en Idamaraș): "je t'avais parlé en ces termes : tu t'en vas, [mais] tu n'as pas du [tout] mis en ordre le pays. Dès ton départ, le pays [devien]dra hostile. Voilà ce que j'avais dit à mon père et mon seigneur, mais il ne m'a pas écoutée. Alors tu m'as répondu en ces termes: $\mathrm{Au}$ milieu du pays, les Hanéens se rassasieront d'orge. Comment pourrais-je donc

${ }^{588}$ Voir aussi 1'"Epopée de Zimri-Lim" où le roi donne l'ordre à son vizir de mobiliser les cheiks afin qu'ils lui amènent les guerriers du Hana", D. Charpin et J.-M. Durand, M.A.R.I. IV, p. 327.

${ }^{589}$ Selon J.T. Luke (Pastoralism, p. 245) et H. Klengel (Zelt und Palast, p. 57), l'alliance assurait aux Hुanéens les droits de pâturage, mais à notre avis, le rassasiement des Hanéens n'a rien à voir avec le pâturage. $\left(^{*}\right)$ 
être négligent envers le p[ays (?)] ?" (X.31.6-16). Par la suite, Kirûm informe Zimri-Lim que "les Hanéens cherchent partout de l'orge, mais il n'y en a pas" (1l. 5'-6'). Nous possédons une lettre qui nous apprend que le don d'orge et de laine aux Hुanéens de la part des rois d'Idamaraş et du Zalmaqum est le résultat des relations de ces rois avec Zimri-Lim. Dans une lettre dont le début manque, nous lisons: "il s'est exprimé en ces termes: Pour [...] et le fait de rassasier les Hanéens [...]. Adal-šenni (le roi de Burundum dans le Zalmaqum) m'a répondu de la manière [suivante:] Maintenant, Nusugga, le roi [de Sinamum,] pour le rassasiement de son district [...] a parlé en ces termes: Que personne ne livre ni orge ni laine aux Ḩanéens. Les trois mille ânes des Hanéens retourneront à vide. Ainsi a (encore) parlé Nusugga, le roi de Sinamum: Pourquoi ton seigneur ne cesse-t-il d'écrire à Adal-šenni, alors qu'à moi il n'a pas écrit ? A cause de cela il ne livrera pas de laine aux Ḩanéens" (B.590.1'-14').590 Un autre témoignage sur l'approvisionnement des Hanéens n'est pas très clair. Un messager de Hāya-Sūmû, le roi d'Ilānșurā, transmettait l'information suivante: "Ḥāya-Sūmû assiège (la ville de) Hazzakannum. En outre, des Ḩanéens portent l'orge de Tādum (en Idamaraş)" (XIV.120.13-16). L'Idamaras était le pays des Hुanéens, ils séjournaient dans les limites des villes-royaumes et étaient même considérés comme fils du district. En effet, Zakura-Abu, le roi de Zalluhān, écrit à Zimri-Lim qu'il propose que les BiniSim'āl aillent "avec les Ḩanéens, les fils de mon district" (B.81.36-38). Des troupeaux des Hanéens paissaient dans le district de Kahat (II.59). Et on trouve aussi un cas où une ville a été donnée aux Hanéens (A.1251.25, 30).(*)

Les Hanéens servaient les divers rois d'Idamaraș. Ibal-El, le merḥum en Idamaraș écrit: "Šadum-Labā (le roi d'Ašnakkum) et Ibal-Addu (le roi d'Ašlakkā) sont arrivés chez moi à Siharatā au milieu des Hanéens" (II.33.11'-13'). C'est peut-être le même merhûm qui doit rassembler, avec Ḩabdu-Malik, sur l'ordre d'Atamrum, le roi d'Andarig, les Hुanéens qui viendront à l'aide d'Atamrum (XXVI.389.9-15; 394.10'-12'). Des Hanéens sont venus avec Qarni-Lim, le roi d'Andarig, pour joindre les rois d'Idamaras (A.1610+.11; A.1212.11) et d'autres Hanéens vont à Kasapā avec Šarrāya, le roi de Razamā, pour faire face à l'ennemi (A. 988.15-16). Deux Ḩanéens ont accompagné Šadum-Labā, le roi d'Ašnakkum, qui se rendait à

${ }^{590}$ Voir II.52.1' -7': 300 ou 400 ânes qui portent de l'orge. 
Mari (III.46). Ašmad du clan Amurrum servait auprès de Hammurabi, le roi de Kurdā (M.6210.8'-9'), et Nuhmuna-Addu du clan Ibalahuu servait auprès de Hammi-Epuh, le roi de Talḩāyum (A.1610+.40-41; A.1212. 38-39). Les Ḩanéens en Idamaraş étaient soumis, à un certain degré, à l'autorité de Zimri-Lim. Ainsi Kibri-Dagān, le gouverneur de Terqa, pouvait amener de là-bas 3000 Huanéens (III.14; XIII.102; 103), et 1000 Hुanéens d'Idamaraş se rendaient avec Ibal-pī-El à Mari (XIV.112.8-9). De même, les Hanéens qui sont installés dans le pays d'Apum se préparent à attaquer une force expéditionnaire de Šeḥnā parce que "Vous (Sehnnā) êtes en guerre avec Zimri-Lim" (XXVI.358.3'). Nous avons vu que les Hanéens venaient en renfort à Mari, mais on trouve aussi le cas contraire, dans un renfort qui avait été envoyé pour aider Kurdā (au sud du Djebel Sindjar), des habitants des villes hanéennes du district de Mari, Appān et Hutnum, et des Yamahamméens. 591

Plusieurs faits nous renseignent sur les relations spéciales qui existaient entre le Palais et les Hanéens. Zimri-Lim proposait aux Hanéens d'accepter un village des Bini-Yamina (BENJ., pp. 984d, e; 989c). Trois prisonnières des Bini-Yamina ont été transférées aux Hanéens (XXI.27+.I.4-8), probablement aux gardes du palais, car ces Hanéens étaient soumis à $\mathrm{Ka}$ 'ala-El, le préposé aux Hanéens de la garde palatine. Le palais a racheté des Hanéens qui ont été faits prisonniers par les BiniYamina (XXIII.540.1-5; M.11351), 592 et un marchand a racheté un habitant de la ville de Nihadû, ville qui porte le nom du clan Nihad (VIII.78). Les Hanéens participaient aux grands repas au palais ${ }^{593}$ et des Hanéens prenaient part aux cérémonies où l'on prêtait serment. ${ }^{594}$ Une fois, le palais a fourni aux Hanéens un boeuf pour un rite (XXI.76), et une autre fois, ils ont participé au culte dans la ville de Terqa (XXIII.91.4-5). Nous trouvons aussi des Hanéens parmi ceux qui envoyaient des dons d'ovins à Zimri-Lim (VII.203. 1'). Les localités ḩanéennes de Yamahammûm et Niḩad ont payé leurs contributions d'orge prélevé sur leurs champs (XXIII.69.9-10). Le palais, de son côté, distribuait des vêtements aux

\footnotetext{
${ }^{591}$ TRUP.I.5, II.7, IV.11, 28, V.14, Tr.lat.II.3.

592Voir XXIII, pp. 515-516.

${ }^{593}$ XXI.83.12'-13'; XXIII.494.3-5, 11; M.13225; M.13244; M.15249.

${ }^{594}$ XXIII.85.1-6; 86.20-43 (?, cfr. p. 77 [I.43]); 87.1-36; 235.III-IV.
} 
Ḩanéens qui revenaient du pâturage, 595 et aux Hanéens qui apportaient des nouvelles. 596 Un Ḩanéen a reçu de l'argent du palais (XXIII.561.1), et un autre a prêté de l'argent à un membre du personnel du palais (XXIII.237.10-13). Un habitant de Niḩad a reçu du palais un objet en bronze (XXII.222.11'-12') et un membre de la tribu Amurrum a reçu un anneau d'or (XXV.100.1-3). Enfin il faut souligner qu'un Ḩanéen du nom de Șabihum était un serviteur du merḥum (XIV.53.6-7). C'est peut-être le même Șabihuum qui a emprunté à Asqudum une somme d'argent dans le "camp" d'Ašlakkā (XXV.740). Un signe d'une certaine tension entre le clan Yamaḩammûm et Zimri-Lim réside peut-être dans le fait qu'après que Zimri-Lim eut frappé les campements des Bini-Yamina, les Yamahamméens sont partis (A.876).

\section{Bini-Yamina}

A. L'époque de Yahdun-Lim - Yaḩdun-Lim a infligé une défaite décisive aux Bini-Yamina et a détruit leurs trois villes-fortifiées: Samānum, Tuttul et Abattum (M.2802.III.3-IV.1), et ainsi il s'empara de toute la vallée de l'Euphrate jusqu'à la ville d'Imār, qu'il a également conquise (N.AN., p. 52, no. 4). Les relations belliqueuses entre Yahdun-Lim et les Bini-Yamina figurent aussi dans l'un de ses noms d'année où on lit: "Année: Yaḩdun-Lim est allé à Ḩên et lui a livré le campement (ou: le pâturage; nawûm) des Bini-Yamina" (N.AN., p. 52, no. 6). C'est sans doute à ses victoires dans la région d'amont que fait allusion la phrase suivante tirée d'une lettre d'Ašmad: "Déjà auparavant, Yaḩdun-Lim contint les pillards et les fit sortir de l'intérieur du pays" (XXVI.24.20-22). Les "pillards" ḩabbātū ne sont autres, pensons-nous, que les Bini-Yaminites.

B. L'époque de Yasmah̆-Addu - Durant l'année éponymale Adad-bani, Yasmah-Addu a remporté une victoire sur les Bini-Yamina, probablement à la porte de Saggarātum (voir ci-dessus Chapitre II, § 2, "L'année Adad-bani"). Et c'est, peut-être, durant cette même année que doivent être placées les lettres II.44;

\footnotetext{
595XXII.147; 171.Rev.4'-11' (= XXIII.446.58' $\left.-65^{\prime}\right)$.

${ }^{596}$ XXII.167.22-25 (= XXII.327.25-28; XXIII.448.24-27). Ce sont peut-être ces mêmes hérauts qui figurent dans une lettre d'Asqudum (XXVI.42).
} 
V.2 qui racontent comment, après sa défaite à Șuprum, Yasmah̆-Addu a néanmoins remporté une victoire sur un ennemi non déterminé, que nous supposerions être les redoutables Bini-Yamina. Presque toutes les autres attestations des Bini-Yamina à cette époque révèlent une coopération entre eux et Yasmah-Addu: par exemple, les Bini-Yamina ont pris part aux guerres menées par YasmahAddu. ${ }^{597}$ La gestion des affaires des Bini-Yamina était soumise aux mains des sugāgū, par l'intermédiaire desquels les autorités géraient les Bini-Yamina; parfois même par des menaces (I.6.18-19). On ne trouve pas à cette époque de rois des Bini-Yamina, car Samsi-Addu ne permettait pas aux Bini-Yamina d'avoir leurs propres rois dans les limites de son royaume. Samsi-Addu voulait avoir des BiniYamina dans son royaume, et pour cette raison il s'abstenait de les recenser craignant le colère des Rabbûm, qui risquaient de ne pas revenir du Yamḩad dans son royaume (I.6.6-13). Il est intéressant de citer à ce propos l'information donnée par $\mathrm{H}$. Charles, $598 \mathrm{~d}$ 'après laquelle au début du 18 ème siècle les membres de la sousfraction $\mathrm{Bu}-\mathrm{L}$ êl, craignant le recrutement qui suivrait le recensement organisé par le commandant turc de Deïr ez-Zôr, ont quitté leur village Qataâ (entre le confluent du Habūr et Deïr-ez-Zôr). Retournons à l'époque de Mari. Pour aider les Rabbûm dans la traversée de l'Euphrate lors de leur retour du Yamb̧ad, Samsi-Addu leur fournissait des bateaux (IV.6). Mais une autre lettre nous révèle une attitude différente envers les Rabbûm: Sîn-tiri va rassembler les Hanéens pour razzier les moutons de Sūmu-Epuh et des Rabbûm (I.24+.40-42). Une autre fois, SamsiAddu s'est même occupé de donner aux Bini-Yamina la possibilité de transhumer librement vers leurs pâturages en amont de l'Euphrate, en veillant à ce qu'ils ne soient pas coupés de ces régions par Sūmu-Epuh, le roi du Yamhad (I. 43.5'-12'), et cela contrairement à l'attitude de Zimri-Lim, comme nous allons le voir par la suite. (*) Les bonnes relations entre les autorités et les Bini-Yamina se manifestent aussi par le fait que Samsi-Addu a conseillé à Yasmah-Addu de se servir des Uprapéens pour conduire un caravane qui partait de Mari pour Qatanum (I.85+.21-22). Une fois seulement on exprime des craintes devant la possibilité d'une razzia des Bini-Yamina. Il s'agit d'une éventuelle razzia de troupeaux des

597I.6.14-20; 42.29-32; 60.9-10.

${ }^{598}$ Tribus moutonnières, p. 8. 
Hanûm-Yamahammûm et des muškēnum (V.81), mais peut-être ne s'agit-il que d'un conflit local. De cette époque, nous n'avons que le témoignage d'un recensement des Bini-Yamina, en l'occurrence dans le pays de Yaḩrurā (II. 18.56 ).

C. L'époque de Zimri-Lim - La description des rapports entre Bini-Yamina et Zimri-Lim - rapports qui ont connu des hauts et des bas - se heurte à une grande difficulté, à savoir le manque presque total de datation des divers événements. Pour cette raison, nous ne savons pas si les relations pacifiques ou belliqueuses entre eux étaient diachroniques ou synchroniques, en se référant aux diverses unités des Bini-Yamina et à des régions distinctes. Plusieurs événements sont datables. Le fait qu'au début de son règne régnaient de bonnes relations entre ZimriLim et les Bini-Yamina est prouvé parce que deux de leurs rois se sont respectivement mariés avec une soeur et une fille de Zimri-Lim. ${ }^{599}$ Les rois, à cette époque, se considéraient comme les frères de Zimri-Lim, c'est-à-dire des égaux, alors qu'il les a considérés comme ses esclaves, serviteurs, donc d'un statut de vassaux inférieurs à lui (A.3274.8'-15'). Mais la paix ne dura pas entre eux et une guerre éclata entre Zimri-Lim et les rois des Bini-Yamina dans le district de Saggarātum (et à Mišlān, ${ }^{600}$ du district de Terqa) au début de l'année ZL 1'601 (N.AN., p. 55, nos. 6-7602), qui a suivi sa mainmise sur la vallée de l'Euphrate dans l'année "Kahat". On peut supposer que les rois des Bini-Yamina sont descendus de la région d'amont, à l'instar de la descente des Bini-Yamina qui, au temps de Yahdun-Lim, sont descendus d'Abattum et de Tuttul à Samānum du district de Terqa. De cette guerre on trouve probablement une allusion dans une lettre qui dit "lorsque les Bini-Yamina ont été hostiles à mon Seigneur" après la conquête de Mari par Zimri-Lim (A.489.5'). Zimri-Lim était aidé dans cette guerre par son allié Hammurabi, le roi de Babylone, qui a envoyé une armée commandée par un

\footnotetext{
${ }^{599}$ D. Charpin et J.-M. Durand, RA LXXX (1986), p. 172, n. 145.

${ }^{600}$ D. Charpin et J.-M. Durand, RA LXXX (1986), p. 144, n. 15.

${ }^{601}$ D. Charpin et J.-M. Durand, M.A.R.I. IV, p. 330: le second mois.

${ }^{602}$ Ajouter peut-être 1'"Epopée de Zimri-Lim", D. Charpin et J.-M. Durand, M.A.R.I. IV, p. 325, n. 139.
} 
ancien Ekallātéen du nom de Mut-Hadkim. ${ }^{603} \mathrm{~J}$.-M. Durand a montré que cinq lettres des archives royales de Mari faisaient partie de la correspondance des BiniYamina qui fut interceptée par Zimri-Lim. ${ }^{604}$ Ces lettres étaient écrites par les serviteurs de Yagih-Addu, habitant de Mišlān (XXVI.169.18; 170.22'; 171. 19') au roi bini-yaminite Sümu-dabi (XXVI.170.10). Elles datent de peu de temps avant l'ouverture des hostilités entre Zimri-Lim et les Bini-Yamina. La période est celle de la moisson (XXVI.168.16; 170.3'), donc les mois de Malkānum (Iyar) et de Laḩhum (Sivân) de l'année ZL 1'. La ville de Mišlān prie Sūmu-dabi de s'adresser aux autres rois bini-yaminites pour qu'ils viennent au secours de Mišlān contre l'attaque de Zimri-Lim (XXVI.168.18-24; 170.9-15, 17'-18'). La ville de Mišlān a à sa disposition mille ou deux mille soldats, et demande une aide d'encore 300 bons soldats (XXVI.171.16', 27'-28'). L'attaque contre la ville de Mišlān était dirigée, semble-t-il, par Ibal-pī-El (XXVI. 172.9-12). Les rois des Bini-Yamina, après leur défaite, se sont réfugiés dans Qatanum, Karkamiš,* puis après l'intervention de Yarīm-Lim, le roi de Ḩalab, ils se sont enfuis au Zalmaqum et ont conclu une alliance avec les rois de ce pays (XXVI.24.6-12). Avec l'aide de leurs alliés, les rois du Zalmaqum, ils ont attaqué de nouveau la région de Mari qui était aidée par Qatanum. Ils ont descendu l'Euphrate et ont attaqué la ville de DūrYahdun-Lim (XXVI.121), mais la seconde attaque n'eut pas, elle non plus, de succès. ${ }^{605}$ (voir ci-dessus Chapitre II, § 3, "L’année ZL 1'"). Dans l'année ZL 4', Zimri-Lim a dû combattre une coalition comprenant Ešnunna, Andarig et les Bini-Yamina. ${ }^{606} \mathrm{~A}$ la suite de la victoire de Zimri-Lim sur les rois des BiniYamina, de nouveaux rois bini-yaminites se sont installés dans leurs villesroyaumes dans la région d'amont, et ils sont devenus les vassaux de Zimri-Lim (BENJ., p. 993b). La paix avec les Bini-Yamina entrainait avec elle la libération de villes des Bini-Yamina (M.6874.4'-5'). Mais l'on craignait sans cesse des hostilités de la part de ces rois (A.82). Et en effet une victoire sur ces nouveaux rois, cette fois dans la région d'amont, fut remportée par l'un des serviteurs de

\footnotetext{
${ }^{603}$ D. Charpin et J.-M. Durand, RA LXXX (1986), p. 171.

${ }^{604} \mathrm{XXVI} / 1$, pp. $335-340$ (nos. 168-172).

605 Voir XXVI/1, p. 139.

${ }^{606}$ D. Charpin et J.-M. Durand, M.A.R.I. V, pp. 632-633.
} 
Zimri-Lim du nom de Yasīm-Maharar (BENJ., pp. 992-993a), mais nous ne savons pas la date de cet événement. En revanche, deux lettres, qui sont à dater de la fin de l'année ZL 9' - début de l'année ZL 10', racontent qu'une guerre va éclater entre lui et les Bini-Yamina. ${ }^{607}$

Quand on étudie les Bini-Yamina et leurs relations avec les autorités, il faut prendre en considération le fait que l'unanimité ne régnait pas toujours entre les tribus qui formaient la confédération tribale bini-yaminite. Parfois des disputes éclataient entre eux, qui aboutissaient même à des hostilités. Par exemple, Șūra-Hammû, le roi des Amnanûm, devait se placer à la tête d'une armée qui était composée des membres de plusieurs tribus, mais il se plaint à Yaqqim-Addu, le gouverneur de Saggarātum: "Où puis-je conduire ces gens, s'il n'y a pas d'accord entre eux ? Certainement à leur perte ! ... Hammi-ištamar (le roi d'Uprapûm) ne cesse de leur écrire, mais personne ne va chez lui" (XIV.83.16-18, 24-26). Et Yaqqim-Addu demande à Șūra-Hammu: "Quand Dādi-Hadun (le roi de Rabbûm) arrivera-t-il ?" (1.30), et il lui répond: "Il viendra" (1. 31). Mais une information lui parvient disant "A propos de ce qu'on t'a raconté, aucun Bini-Yaminite ne viendra. Quant à Dādi-Hadun, il est en train de faire moissonner son grain à Abattum" (11. 34-37). Parfois les rois se disputaient entre eux. Une lettre écrite probablement par YarīmLim (le roi des Yahrurûm) nous décrit des relations tendues entre les rois: "C'est du beau ce que Șūra-Hammû [a fait] ! Au sujet du rapport que [j'avais exposé] devant mon seigneur, il (Șūra-Hammû) a écrit à Dādi-Hadun (le roi de Rabbûm) en ces termes: ... D'autre part, ici, devant Zimri-Lim, pour dire du mal de ton serviteur, Yarīm-Lim s'est présenté et sur son ordre on a emprisonné ton serviteur. Ecris pour qu'on pille 3 ou 4 parcs à moutons des Yahrurûm, et fais passer les moutons au-delà du fleuve. Ces choses-là et beaucoup d'autres désagréables, Șūra-H̆ammû [les a dites] à Dādi-Huadun" (A.3821.5-10, 14-24). Nous voyons donc qu'un roi des Bini-Yamina se plaint d'un autre roi des Bini-Yamina devant Zimri-Lim. (*) Il arrive aussi que les rois des Bini-Yamina présentent leur litige à Zimri-Lim pour qu'il tranche l'affaire: Yasmaḩ-Addu, le roi de Yarihûu, prie Zimri-Lim: "[Lorsque] (Hammi-ištamar, le roi d'Uprapûm) présentera son [affai]re devant toi, [ne sois pas d'ac]cord avec lui, réponds-lui ainsi: Jusqu'à ce

${ }^{607}$ BENJ., p. 990b, 11. 21'-27'; AREP., p.108 + BENJ., p. 991a, 11. 26-30. 
que Yasmaḩ-Addu vienne ici, ne pr[ésente] pas son affaire" (II.54.8'-12'). On trouve aussi des cas dans lesquels une partie des Bini-Yamina, ceux qui demeurent dans les districts, étaient en bons termes avec les autorités, tandis que leurs frères qui habitaient la région d'amont étaient hostiles au pouvoir central. ${ }^{608}$ Pour cette raison, nous ne savons pas si une information portant sur un conflit entre Zimri-Lim et les Bini-Yamina signifie qu'il s'agit d'une guerre générale avec toutes les tribus, ou peut-être seulement d'un conflit avec une unité tribale qui se trouve dans une région déterminée. Il est possible qu'à la vérité, on doive compter parfois avec des conflits généraux et parfois avec des conflits locaux. Les Bini-Yamina, comme tous les nomades pratiquaient assez souvent la razzia. Dans la société bédouine, la razzia ( $g a z \bar{u})$ est une occupation honorable, ${ }^{609}$ et c'était un privilège que d'y prendre part. Le but principal de ces razzias était les troupeaux de l'ennemi, et par ce moyen d'agrandir ses propres troupeaux. Ils sortaient annuellement faire des razzias qui pouvaient durer des semaines ou même des mois. Souvent la razzia était conduite par le cheikh, et parfois par un dirigeant spécial qui porte le nom de 'aqid. Les autorités faisaient évidemment de leur mieux pour les empêcher de pratiquer cette tradition. $H$. Charles ${ }^{610}$ raconte qu'au début du mandat français, les 'Agêdât pratiquaient beaucoup les razzias, et pour pacifier la vallée de l'Euphrate, les Français envoyaient contre eux une force militaire. De même, à l'époque de Zimri-Lim, les autorités ont souffert assez souvent des razzias des BiniYamina: "Depuis le jour où je me suis rendu à l'Ah-Purattim, les Bini-Yamina se sont mis à razzier. Une première fois, ils ont razzié et ils ont pris de nombreux moutons. J'ai envoyé à leur poursuite des troupes de secours et elles leur ont infligé une défaite; pas un seul d'entre eux n'y a échappé et ils ont rendu tous les moutons qu'ils avaient pris" (BENJ., p. 988c, 11. 4-9). ${ }^{611}$ Les Bini-Yamina ont pratiqué souvent aussi des razzias dans la région de Tuttul (BENJ., p. 987d), et aussi contre la ville de Tuttul elle-même (A.215). Les Bini-Yamina se sont ralliés aux

\footnotetext{
${ }^{608}$ Voir XIV.89 et aussi III.16. $\left({ }^{*}\right)$

${ }^{609}$ Voir M.P. von Oppenheim, Die Beduinen, p. 34; V. Müller, Bédouins, pp. 156 ss.;

L. Stein, Die Schammar-Gerba, pp. 133-134, 137.

${ }^{610}$ Tribus moutonnières, p. 12.

${ }^{611}$ Voir aussi BENJ., p. 988a.
} 
rois du Zalmaqum pour attaquer la ville de Dīr (XXVI.24). ${ }^{612}$ Zimri-Lim a été averti par deux fois de la possibilité d'attaques des Bini-Yamina sur des campements (BENJ., pp. 987b, 987d-988a). Nous possédons aussi une information d'après laquelle les Bini-Yamina ont eu l'intention de razzier les troupeaux des rois d'Idamaraș. Pour se défendre contre cette attaque, un de ces rois, Sibkuna-Addu, le roi de Sudā, a conclu une alliance avec Zimri-Lim et les Bini-Sim'āl. Cette alliance leur permettra de razzier le territoire des Bini-Yamina dans le Zalmaqum (A.1208). C'est peut-être sur ce fond qu'il faut comprendre une phrase citée par G. Dossin: "Et si mon seigneur veut emmener ses troupes contre les BiniYamina, que mon seigneur écrive aux rois d'Idamaraş" (BENJ., p. 992a). (*)

Les razzias des Bini-Yamina ne.se font pas à l'improviste, mais elles sont le résultat de consultations dans leurs conseils de guerre (XIV.84+.4-11). Dans cette lettre, on lit qu'après s'être aperçus que l'Aḩ-Purattim s'apprête à se défendre contre leur attaque, ils se dirigent vers la ville d'Abattum pour la conquérir (11. 4547). Dans un autre cas, ils ont l'intention d'attaquer le district de Qattunān (BENJ., p. 989b). Et plus au sud, ils ont assailli trois gardiens des champs près de la ville de Samānum (III.16.20-26). Dans le district de Saggarātum "Les Uprapûm ont pillé trente arpents de la meilleure terre" (XXVI.462.17-18). Quand l'occasion s'est présentée, les Uprapéens ont attaqué une caravane qui se rendait de Karkamiš à Mari (XIV.86.4-16) et par une action de représailles, les gens de Zimri-Lim ont razzié les troupeaux uprapéens (11. 17-24). (*) Zimri-Lim lui-même a attaqué un campement des Bini-Yamina (A.876.9'-11') et il a ordonné de détruire les parcs à moutons et les troupeaux des Hanûm-Bini-Yamina (A.1086).

A propos des Bini-Yamina comme à propos des Hanéens, on trouve le terme de rihşum "palabre". Les Anciens des Bini-Yamina palabrent avec quelqu'un probablement au sujet de la libération de leurs villes (M.6874). Dans un autre cas, c'est le préfet de Tuttul, Lanasûm, ${ }^{613}$ qui palabre avec des Uprapéens à propos de leur transhumance (A.4530-bis). Le même Lanasûm écrit que les rois du Zalmaqum et les Bini-Yamina se concertent pour conquérir la ville de Tuttul (A. 215)

${ }^{612}$ Voir aussi BENJ., p. 986 b.
${ }^{613}$ Cfr. J.-M. Durand, XXVI/1, p. 182. 
Les conflits permanents entre les Bini-Yamina et Zimri-Lim expliquent son attitude préférentielle envers les Hुanéens. Il suggère aux Hुanéens d'accepter un village des Bini-Yamina et de manger leur orge, ${ }^{614}$ il rachète des Hanéens qui ont été capturés par les Bini-Yamina ${ }^{615}$ et il transmet aux Hanéens des prisonnières biniyaminites. ${ }^{616} \mathrm{Ce}$ sont les Hanéens qu'on veut envoyer pour défendre Dìr du Balih contre une attaque des Bini-Yamina et des rois du Zalmaqum (XXVI.24.17$20,23)$.

Dans leur guerre contre Zimri-Lim, les Bini-Yamina se sont joints à Qarni-Lim, le roi d'Andarig, en concluant avec lui une alliance. ${ }^{617}$ Dans un autre cas, on parle d'une éventuelle union entre les Bini-Yamina et Ešnunna (BENJ., p. 991d.15'17'), 618 et d'une triple union: Ešnunna, Qarni-Lim et les Bini-Yamina (Ch.-F. Jean, EXCE. 1939, no. 702, 11. 6-11). ${ }^{619}$ Ailleurs, une lettre de Lanasûm, le préfet de Tuttul, informe Zimri-Lim que les Bini-Yamina et le Zalmaqum se sont rencontrés en vue de conclure une alliance (A.954). Et une autre lettre, cette fois de Yasmahy-Addu, le roi de Yarihûm, nous fait savoir que les rois du Zalmaqum et les rois des Bini-Yamina palabrent entre eux à Zalpah (A.2526). De même, on voit que les Bini-Yamina s'efforçaient de conclure une alliance avec le roi AdūnaAddu (un roi d'Idamaraș) (BENJ., p. 990a). Les Bini-Yamina ont suggéré aux gens de la ville de Narā (dans le district de Saggarātum) de se joindre à eux pour conquérir le pays de ce même Adūna-Addu (S.115, no. 72-17, 11. 7-8). Les BiniYamina qui habitaient dans la région d'amont ont montré un grand intérêt pour les affaires de Tuttul, une ville soumise à Zimri-Lim. Yasmah-Addu, le roi de Yarihûm, était mêlé à une intrigue qui visait à évincer Lanasûm, le préfet de la ville (II.137). Et les Uprapéens, qui contrôlaient l'eau irriguant les champs de Tuttul,

${ }^{614}$ BENJ., pp. 984d, e, 989c.

615XXIII.540.1-5; M.11351.

${ }^{616}$ XXII.27+.I.4-8.

${ }^{617}$ BENJ., p. 990b, 991a.

${ }^{618} \mathrm{La}$ lettre A.2741 est liée peut-être à la même affaire.

${ }^{619}$ Sur un lien entre Bini-Yamina et Ešnunna on peut, peut-être, invoquer le témoignage d'un document datant de l'année de la victoire de Zimri-Lim sur les Bini-Yamina où on mentionne la libération des hommes d'Ešnunna et de la tribu Amnanûm (XXI.321.1-7). 
ont forcé les habitants de Tuttul à changer d'avis dans une certaine affaire (A.2769). Enfin, Șüra-Hammû, le roi d'Amnanûm, Yarìm-Lim, le roi de Yahrurum et Dădi-Ḩadun, le roi de Rabbûm, étaient en relation d'une façon ou d'une autre avec le culte de Dagān à Tuttul (VI.73).

Jusqu'à maintenant, nous avons connu les Bini-Yamina comme adversaires des autorités, mais maints exemples montrent qu'ils pouvaient aussi très bien collaborer avec le pouvoir central et s'intégrer aux affaires de l'Etat. Tout d'abord, il faut signaler que les rois des Bini-Yamina, qui régnaient dans la région d'amont, étaient des vassaux de Zimri-Lim (voir ci-dessus Chapitre V, §§ 1, 3). Ils fréquentaient souvent Mari et maintenaient un contact permanent avec la ville par l'intermédiaire de leurs messagers. ${ }^{620}$ Les rois ont visité aussi les districts de Saggarātum et de Terqa, peut-être en se rendant à Mari (voir ibidem). Ils sont venus aussi à Mari pour régler les conflits qui éclataient entre eux (voir ibidem). Les rois, comme les autres vassaux de Zimri-Lim, ont mis leur gens à la disposition de Zimri-Lim (voir ibidem), ont envoyé des moutons au palais de Mari (VII.226. 33, voir ibidem), et ils y ont même envoyé des cadeaux (II.104), ils ont également transmis au palais de Mari les taxes prélevées sur leurs champs (II.61 et voir ibidem). Yasmah-Addu, le roi de Yarihûm, a emprunté de l'argent à Zimri-Lim (VIII.37; [42]), et une fois il a reçu du palais de Mari de l'argent et des moutons (IX.23). Lors de son voyage à Ugarit, Zimri-Lim a reçu des cadeaux de la part de Dādi-Ḩadun, le roi de Rabbûm (voir ci-dessus Chapitre II, § 3, "L'année ZL 8' "), et la même année il a envoyé des cadeaux à Hुammi-ištamar, le roi d'Uprapûm. ${ }^{621}$ La coopération entre les rois et Mari se manifeste aussi par le fait que des gens de Șūra-Ḩammû ont participé à un acte juridique qui était dressé à Mari (VIII.67). Parfois on constate que les relations entre les rois et le palais étaient particulièrement étroites: Dādi-Hูadun réconciliait Šibtum, la femme de Zimri-Lim, avec son frère Hammurabi, le roi du Yamhad (X.156).

Etant des vassaux de Zimri-Lim, les rois des Bini-Yamina avaient un statut plus élevé que celui des gouverneurs des districts. Par exemple, Yaqqim-Addu, le gou-

\footnotetext{
${ }^{620}$ VII.159.14-15; 164.9-10; 165.10; 210.16'-18'; 211.15-17; 212.19-20; XXIII.241.1-5. ${ }^{621}$ S.143, no. 132 (le nom du roi est suivi par un point d'interrogation); M.18229 (du IX ${ }^{\text {ème }}$ mois, sans année).
} 
verneur de Saggarātum, craignant la colère de Șūra-Ḩammû, le roi d'Amnanûm, a envoyé à sa rencontre un mouton et une jarre de vin pour apaiser sa colère (XIV.83.9-12). De même, Hammi-ištamar, le roi d'Uprapûm, explique à KibriDagān, le gouverneur de Terqa, qu'il ne lui livrera pas deux habitants de Terqa, dont Kibri-Dagān demande l'extradition, car ils sont innocents (II.94). Par contre, Kibri-Dagān écrit à Zimri-Lim à propos d'un homme qui s'enfuit de chez Hammiištamar et trouva un asile à Terqa (III.71). Quand une dispute éclata entre ȘūraHammû et Kibri-Dagān, on présenta l'affaire devant Zimri-Lim pour qu'il la tranche (III.36). Et nous voyons que Kibri-Dagān attend l'arrivée de Dādi-Hadun, le roi de Rabbûm, avant d'offrir des sacrifices au dieu Adad (III. 45).

Voyons à présent quel était le statut des Bini-Yamina qui habitaient le royaume de Zimri-Lim: Kibri-Dagān, le gouverneur de Terqa, a recensé les Bini-Yamina qui se trouvaient dans son district (III.21), et Yaqqim-Addu, le gouverneur de Saggarātum, a recensé les Amnanéens de la ville de Saḩrū (XIV.64.10'-14'). Le recensement est le signe par excellence de la soumission des membres d'une tribu à l'autorité du gouverneur. Dans des listes des hommes et femmes qui ont pris part aux cérémonies de prestation de serment, on trouve, entre autres, les habitants des localités des Bini-Yamina. ${ }^{622}$ Les membres des tribus qui séjournaient dans les districts de Terqa et de Saggarātum maintenaient des contacts permanents avec la région d'amont où ils montaient à l'époque de la transhumance (III.58), mais les gouverneurs s'efforçaient d'empêcher leur lien avec la région d'amont. ${ }^{623} \mathrm{~J}$.-R. Kupper ${ }^{624}$ suppose que Zimri-Lim s'opposait seulement à de grandes migrations, car elles pouvaient entraîner avec elles pas mal de troubles. On peut citer à titre

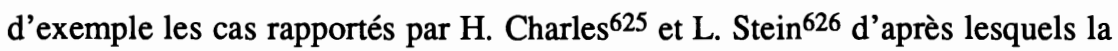
transhumance était une source permanente de soucis pour les autorités. H. Klengel, ${ }^{627}$ quant à lui, pense que la raison de l'objection de Zimri-Lim réside

${ }^{622}$ XXIII.86; 593; IX.291(en comparant avec XXIII.236).

${ }^{623}$ II.92; 102; III.12.16-26.

${ }^{624}$ Nomades, p. 65.

${ }^{625}$ Tribus moutonnières, p. 15.

${ }^{626}$ Die Schammar-Gerba, p. 101.

${ }^{627}$ Benjaminiten und Hanäer, p. 224; Zelt und Palast, p. 58. 
dans le fait qu'il voulait les empêcher de fuir leurs devoirs envers les autorités. On peut aussi se demander si l'objection de Zimri-Lim ne se limite pas aux époques durant lesquelles les Bini-Yamina qui résidaient dans la région d'amont lui étaient hostiles (III.16; XIV.89). Les rapports entre le gouverneur et les Bini-Yamina sont entretenus par les sugāgū (II.92) qui sont responsables de la bonne conduite de leurs gens et par les Anciens qui représentent les membres de la tribu (II.83). Les Bini-Yamina résidaient dans des villes des districts et ils possédaient des champs qu'ils cultivaient (voir ci-dessus Chapitre VI, § 3B). Dans les districts, on trouve des régions qui sont réservées au pâturage des Bini-Yamina, et YaqqimAddu, le gouverneur de Saggarātum, veillait à conserver leurs droits de pâturage. ${ }^{628} \mathrm{Il}$ arrive que les troupeaux des Bini-Yamina entraient dans un district sans l'accord du gouverneur, et cela provoquait la crainte d'éventuels troubles (II.90). Les bonnes relations qui existaient entre les Bini-Yamina et le pouvoir central pouvaient se transformer subitement. Par exemple, les Bini-Yamina se sont révoltés et ont causé des tels soucis à Zimri-Lim qu'il avait peur de sortir de Mari. ${ }^{629}$ En outre, une lettre nous apprend que, quand les Bini-Yamina sont arrivés à Saggarātum, la déesse Dīrītum a conseillé à Zimri-Lim de ne pas conclure une alliance avec eux (XXVI.199.33-35).

Les Bini-Yamina qui séjournaient dans les districts du royaume étaient soumis aux mêmes obligations que les autres habitants du district. Entre autres, ils devaient fournir des hommes pour les expéditions royales. Par exemple, trois lettres, un document administratif et son duplicata traitent d'un recensement qu'a effectué Bahdi-Lim, le gouverneur de Mari, à Dīr, au sud de Mari. ${ }^{630}$ Bahdi-Lim a recensé les Bini-Yamina qui se rassemblaient à Dīr venant de leurs villes des districts de Mari, de Terqa et de Saggarātum. Dans ses lettres, Baḩdi-Lim écrit qu'il manque encore un grand nombre d'hommes et qu'il s'occupe à remplir le quota imposé aux sugāgū. Le document démontre concrètement les paroles de Bahdi-Lim. Sur 354 hommes qui auraient dû venir à son appel, sont venus seulement 145 (un quart), et 209 (deux tiers) ne sont pas venus (XXIII.429.38). Les hommes re-

${ }^{628}$ BENJ., p. 986, n. 1; XIV.85.

${ }^{629}$ SIGN, pp. 178, 181a.

${ }^{630}$ VI. $30 ; 32 ; 38 ;$ XXIII.428; 429. 
présentaient la réserve (1. 1), qui devait être envoyée en Babylonie (1. 39). Le document date du 12 du second mois de l'année $Z \mathrm{ZL} 10$ '. On sait bien qu'à l'année ZL 9', Zimri-Lim a envoyé une armée à l'aide de Hammurabi, le roi de Babylone. Cette armée était composée essentiellement de Hanéens. ${ }^{631}$ Elle est retournée à Mari vers l'hiver de la même année, car Zimri-Lim en avait besoin pour son expédition en Idamaraş. A la place de cette armée, Zimri-Lim a envoyé au début de l'année ZL 10' une nouvelle armée qui comprenait des Bini-Yamina, la réserve. De même, on constate que des Bini-Yamina ont été envoyés aussi comme renfort à Kurdā, car on trouve dans la liste des envoyés des habitants de Dabiš, une localité bini-yaminite du district de Saggarātum (TRUP.IV.5), et dans une autre liste de gens de l'armée, nous trouvons des habitants de villes bini-yaminites des districts de Mari, Terqa et Saggarātum: Dìr, Raqqum, Ilum-Muluk, Dabiš, Samānum et Narā. 632 .

Les Bini-Yamina qui devaient fournir, comme les autres habitants du district, des gens pour creuser des canaux, n'ont rempli que la moitié du quota désiré (III.6. 519), et ceux qui devaient aider à la moisson du blé des champs royaux ne sont pas venus du tout (III.38.24-26). En outre, ils devaient préparer des habits pour le palais (III.70). Et dans une liste des habits qui étaient fabriqués par des villes du district de Saggarātum, on trouve la ville de Zarri-Rabbiyum (XXII.121.3-4). Les Bini-Yamina étaient soumis aussi aux taxes (II.137.26-29), et le pouvoir central prélevait des taxes sur leur blé.633 De même, une taxe était imposée sur les marchandises importées (XIII.86.10-12). Le palais, de son côté, a accordé des objets en bronze à un Amnanéen et à un Yarihéen (XXII.203.IV(*); 222. 1'-4'), un vêtement à un Uprapéen (XXIII.14) et dix ovins à un autre Uprapéen (XXII.292.7-8). Enfin, parmi les localités qui ont reçu des prêts d'orge, on trouve aussi celles qui appartiennent aux Bini-Yamina (XXIII.70).

Quand on veut résumer l'intégration des Bini-Yamina dans les affaires de l'Etat, on constate qu'on se méfiait d'eux. Ils étaient obligés de s'intégrer dans le district, mais en cas de refus, on obtenait leur coopération par des menaces, et ils pouvai-

\footnotetext{
${ }^{631}$ II. $22-23 ; 25$.

${ }^{632}$ XXIII.596.I.12', II.8', 14', 16', 19', III.10, 23, IV.8.

633 BENJ., p. 985b; XXIII.69.5.
} 
ent toujours surprendre les autorités par une insurrection soudaine. Et on ne doit pas oublier qu'ils avaient une double allégeance - envers le royaume où ils habitaient et envers les frères qui habitaient la région d'amont. 


\section{Bini-Sim'ål}

A. L'époque de Yahdun-Lim - Il semble que Yaḩdun-Lim ait octroyé aux Bini-Sim'āl le pays de Yapturum et la ville de Talhāyum (XIII.144.26-29), mais on peut aussi comprendre que le pays et la ville étaient entre leurs mains. ${ }^{634}$

B. L'époque de Yasmah̆-Addu - Samsi-Addu a demandé l'aide des BiniYamina et des Bini-Sim'āl dans son expédition au Zalmaqum (I.60.9-10).

C. L'époque de Zimri-Lim - Tout d'abord, il faut signaler que Zimri-Lim luimême était considéré par Hammurabi, le roi de la Babylonie, comme "le roi des Bini-Sim'āl" (XXVI.385.6'). Ce fait explique peut-être pourquoi les Bini-Sim'āl étaient très probablement soumis à l'autorité du merhum (préposé au pâturage royal) Ibal-El (voir ci-dessus Chapitre IV, § 6c). Comme à l'époque de YahdunLim, on leur a octroyé le pays de Yapturum et la ville de Talhâayum (XIII.144. 2629). De même, on voit un lien entre Zimri-Lim et les Bini-Sim'āl dans le fait que Sibkuna-Addu, le roi de Sudā, a conclu une alliance avec les Bini-Sim'āl et avec Zimri-Lim (A.1208). Ce lien apparaît aussi dans une lettre de Zakura-abu, le roi de Zalluhān, qui demande à Zimri-Lim d'envoyer les Bini-Sim'āl chez Ibal-pī-El (le merhum [?]), pour qu'ils partent avec les Hanéens (B.81.36-38). Des membres de la tribu ont participé à la garde de la ville de Terqa (XXVI.144.19'-21'). Les bons rapports des Bini-Sim'āl avec le palais se voient aussi dans le fait qu'ils ont envoyé huit jarres de vin au palais (IX.15.6-7), et que le palais a racheté une femme bini-sim'ālite (XXV.130.1-3). L'intégration de la tribu dans la vie du royaume se manifeste par les faits suivants: une femme de la tribu est devenue une prêtresse de la déesse Annunītum (X.59.3'-4'), et des membres de la tribu remplissaient la fonction de devin auprès de Zimri-Lim (XXVI.5.30, 46-48). Enfin, il faut signaler que les Bini-Sim'āl ont aussi conclu une alliance avec les Anciens de la ville de Hurrā en Idamaraș (A.641).

On voit donc que la tribu entretenait de bonnes relations avec tous les rois de Mari.

${ }^{634}$ Cfr. M.B. Rowton, JNES XXXII (1973), p. 212. 


\section{Sutûm}

A. L'époque de Yahdun-Lim - Trois documents ${ }^{635}$ attestent l'existence de la taxe (miksum) que les Sutûm ont prélevée sur leurs troupeaux.

B. L'époque de Yasmah-Addu - A cette époque on constate que YasmahAddu a maintenu de bonnes relations avec un dirigeant sutéen du nom de Hुammitalû, qui servait de guide à une caravane allant de Mari à Qatanum (I.17). Il est possible qu'il s'agisse ici d'une coutume nomade d'après laquelle chaque tribu était responsable de la sécurité d'une caravane qui traversait son territoire et devait fournir des gens pour l'accompagner. ${ }^{636}$ En outre, nous trouvons des Sutéens mêlés à des transactions juridiques à Mari: un esclave sutéen a été acheté à son maître, Sutéen lui aussi (VIII.9+20), et un autre Sutéen a reçu de l'huile du palais de Mari (TH.82.86). Mais nous apprenons aussi que des Sutéens ont fait des razzias sur Rapiqum et Yabliya (I.83; 100), ${ }^{637}(*)$ sur le campement d'un allié de Yasmah̆-Addu, Išhi-Addu, le roi de Qatanum, et sur les villes de Tadmer et Našalā (V.23).

C. L'époque de Zimri-Lim - A cette époque aussi, on constate que ZimriLim était en bonnes relations avec les Sutéens: des Sutéens qui servaient comme messagers obtenaient des payements pour leurs commissions: de l'argent, ${ }^{638}$ des vêtements ${ }^{639}$ et de l'huile (VII.19). Un document attire tout particulièrement l'attention, on y lit que des vêtements ont été livrés à un Sutéen qui remplissait la fonction de grand prêtre de la déesse Nin-Nagar (XXI.370.6'-7'). A un dirigeant sutéen on envoyait un vêtement et un arc (XXI.170.6-8). Deux prisonnières biniyaminites sont livrées aux Sutéens (XXII.27+.I.21-23). Et nous trouvons même

${ }^{635}$ T.179; T.178; T.338 (XXVI/2, p. 13).

${ }^{636} \mathrm{H}$. Charles, Tribus moutonnières, p.11.

${ }^{637}$ D'après J.T. Luke (Pastoralism, p. 134, n. 71 ) les Sutéens n'ont pas razzié les villes ellesmêmes, mais seulement les troupeaux qui se trouvaient dans les environs de ces villes.

${ }^{638}$ VII.133.1-3; 165.15; 169.11-13; 210.19'-21'; XXIII.241.9-11.

${ }^{639}$ VII.110.4'; XVIII.61.21-23 (=XXII.167.17-19; 327.19-21); XVIII.64; XXI.336; XXII.327. 29-35; XXIII.446.21'-29'; 448.19-21, 28-34. 
un Sutéen qui possède un sceau-cylindre (VIII.9+20). ${ }^{640}$ Hammurabi, le roi de Babylone, se sert d'un Sutûm pour envoyer une lettre à Baḥdi-Lim, le gouverneur de Mari (VI.51.13'-20'). On trouve même que le pouvoir central a prélevé des taxes sur les troupeaux des Sutéens (IX.244), une opération qui symbolise bien la soumission des nomades aux autorités centrales. ${ }^{641}$ De même, ils ont envoyé des moutons au palais (VII.225.20). Par contre, Bahdi-Lim fournissait à Hammi-talû, le dirigeant sutéen, environ 40000 litres de blé (VI.15.18-22).642 La raison de la fourniture de cette grande quantité de blé pourrait être la même que dans le cas des Hanéens d'Idamaraş - pour les apaiser. On trouve aussi des Sutéens à la cour de Zimri-Lim. Ces Sutéens ont révélé des secrets du palais à un roi du nom d'IšarLim (II.124.15-18). On trouve des Sutéens aussi à la cour d'un autre roi, Simahlānē, le roi de Kurdā, qui lui-même était d'origine numhéenne (A.2801.1419). Enfin, des Sutéens ont acheté des esclaves, peut-être des mains des Hanéens (XIV.79). De même qu'à l'époque de Yasmah-Addu, à l'époque de Zimri-Lim aussi nous constatons qu'à côté des activités pacifiques, les Sutéens ont agi aussi comme adversaires des autorités. Ils ont attaqué les villes de Ayabū et de Harbē qui se trouvent dans la région de Suhum, au sud de Mari (XXIII.592.4-6). Buqāqum, qui était gouverneur dans une ville de la même région, écrit que "les Sutéens ont fait une razzia dans le pays (?), je suis allé à la rescousse et j'ai ramené les moutons" (XXVI.483.3-5). Menih̆um, un autre gouverneur d'un district de la même région, ${ }^{643}$ écrit à Bahdi-Lim, le gouverneur de Mari, à propos d'une menace de razzia par 30 Sutéens. Les Sutûm visent les troupeaux, pour cette raison Menihum ordonne aux autres gouverneurs de la région de faire passer les moutons à la Djéziréh (qaşûm) (VI.57; 58). Il arrive aussi que les Sutûm enlèvent des gens. En l'occurrence, il s'agit du serviteur d'un personnage de Terqa et de deux vieilles personnes (ou: témoins) (XIII.106. 14-19). Buqāqum informe ZimriLim sur une razzia des Sutûm dans la région d'Agadé qui se termina par un échec

\footnotetext{
${ }^{640}$ Cfr. M.A.R.I. II (1983), p. 62.

${ }^{641} \mathrm{Cfr}$. H. Charles, Nomades moutonniers, p. 15.

${ }^{642}$ Voir J.-R. Kupper, Nomades, p. 85; J.T. Luke, Pastoralism, p. 120.

${ }^{643}$ Cfr. VI.58.21: "mon district", d'après J.-M. Durand (M.A.R.I. V, pp. 665-666) il était un haut fonctionnaire de Zimri-Lim.
} 
total (XXVI.482). A ce propos, un rêve de Zimri-Lim est très significatif. Le roi a rêvé d'un danger qui menace l'auteur de la lettre et la femme du roi, Dam-ḩurāşi. Les Sutéens disent dans le rêve: "Tant que tu ne rendras pas nos demeures, nous ne les libérerons pas" (XXVI.225.10-12). En général, l'apparition des Sutéens dans un district, de même que l'apparition des Bini-Yamina, suscitait l'inquiétude chez le gouverneur (III.12).

En résumé, on voit donc que l'attitude des Sutûm envers les autorités n'était pas uniforme et cela en raison de leur grande dispersion, leur nomadisme et l'absence d'une autorité centrale. De sorte que chaque unité tribale menait sa diplomatie indépendante.

\section{Yamutbal}

A. L'époque de Zimri-Lim - D'après une lettre d'Asqudum, nous apprenons que Zimri-Lim voudrait conclure une alliance avec la tribu Mutibal (= Yamutbal) (XXVI.39.13-25). Dans les renforts qui ont été envoyé de Mari à Kurdā, on trouve des hommes de la tribu Yamutbal (TRUP.V.9, VI.12). Un autre Yamutbaléen qui faisait partie de l'armée d'élite a déserté de l'armée (XXIII.433.III. 4). D'après une lettre du commandant Yassi-Dagān écrite à son maître Zimri-Lim, des membres de la tribu qui se trouvaient dans le palais de Subat-Enlil ont été garrottés par Bunu-Ištar, le roi de Kurdā (II.130.16-18). Cet événement doit être mis en relation avec le fait que Ḩatnu-rabi, le roi de Qațtarā ou Karanā, l'allié de Bunu-Ištar, a pillé le Yamutbal (A.505). Les membres de la tribu font partie de l'armée d'Atamrum, le roi d'Andarig (XXVI.394.20'-21'). Enfin, on trouve des membres de la tribu qui ont quitté le cadre tribal pour joindre les marginaux, les ḩabirū (A.2939).

\section{Numb̧ā}

A. L'époque de Yah̆dun-Lim - Deux hommes de la tribu Numḩā ont reçu des vêtements du palais de Mari (XXII.140; 164.7-9).

B. L'époque de Zimri-Lim - Simaḩlānē le Numahéen est parvenu à la royauté de Kurdã à l'aide de 150 soldats babyloniens et 50 numhéens (cfr. ci-dessus 
Chapitre V, § 1c). Nous trouvons aussi les membres de la tribu Numḩā dans l'armée de Bunu-Ištar, le roi de Kurdā, qui les a envoyés contre Qarni-Lim, le roi d'Andarig (II.130.11-13), et chez Aškur-Addu, le roi de Karanā (XXVI.394. 22'23'). Le commandant Kukkatānum s'adresse aux soldats numb̧éens qui se sont rassemblés à Qațarā, les incitant, sans succès, à la rébellion (XXVI.412.7-16). Par contre, à une autre époque, un employé de Zimri-Lim a conclu une alliance entre le [Numb̧ā], Yamutbal et Qarni-Lim (X.157.12-16). Une victoire de ZimriLim sur les Numhāa est supposée par le fait que, dans l'année ZL 7' Zimri-Lim a reçu de Ḩammurabi, le roi de Kurdã " 2 mines (= $1 \mathrm{~kg}$ ) d'argent (prix du) rachat des hommes de Numhā" (IX.48.1-3). Si le prix de rachat ici est le même que le prix de rachat des Bini-Yamina, qui était de 8 sicles (= $66.6 \mathrm{gr}$ ) par personne (XXII.262.VI.24-26), on a affaire ici à 15 prisonniers. Des activités belliqueuses des Numhā sont mentionnées dans un mémorandum qui concerne l'Idamaraș. Sont enregistrées dans ce mémorandum une razzia dans le pays de Numb̧ā, peutêtre exécutée avec l'aide de soldats hanéens, et une grande razzia faite par les Numhéens, qui demandait la coopération de plusieurs rois pour y résister (A. 3209.17-22). C'est, peut-être, à l'hostilité de la part des Numhéens que se réfèrent les paroles d'Asqudum: "Les Numhéens conduiront les esclaves, les bovins et les ..." (XXVI.41.1'-2'). Mais les textes nous montrent aussi le côté pacifique des Numhéens. Un campement de Zimri-Lim paissait entre le Numhā et le Yamutbal (VI.42.18-19), deux des membres de la tribu ont reçu des vêtements du palais ${ }^{644}$ et un autre homme a reçu du palais des objets en bronze (XXII. 222.11'-12'). Enfin, un Ešnunnéen du nom de Șilli-Ištarān accompagné de Yanșib-Addu le Numbéén transportait la vaisselle de ce dernier à Ešnunna (XXVI. 6.66-68), et lors d'une autre occasion ce même Yanşib-Addu avec ses gens aide Asqudum dans le transport d'un objet pesant (l'alum) à Halab (XXVI.18.40-42).

${ }^{644}$ XXIII.448.50-52 $=$ XXII.150.Rev.1-3. 


\section{Chapitre VIII: L'origine des tribus amurrites}

Maintenant, après avoir étudié les tribus amurrites de Mari, nous pouvons examiner les deux théories que nous avons exposées dans l'introduction à notre étude. La différence essentielle entre les deux réside dans la question suivante: les tribus amurrites ont-elles pénétré dans le pays habité venant de la steppe ou au contraire, ont-elles séjourné dans le pays habité et en bordure des déserts depuis toujours ?645 En d'autres termes, est-ce que les tribus étaient composées à l'origine de nomades moutonniers qui ont adopté l'agriculture après avoir pénétré dans la zone agricole ou au contraire, sont-elles formées de villageois agriculteurs qui ont pratiqué aussi l'élevage ? Quand on essaie de répondre à cette question, il faut se rappeler que le laps de cinquante ans qu'a duré l'époque des archives de Mari reflète un état statique, ${ }^{646}$ et nous devons essayer d'examiner s'il existe des témoignages d'époques antérieures à l'époque des archives. Pour atteindre ce but, nous devons élargir le cadre de notre discussion en ce qui concerne la chronologie et la géographie.

Le seul indice sur la composition de la population du royaume de Mari est basé sur l'examen des noms propres. D'après $C$. Rasmussen, le partage des noms propres est le suivant: $40.4 \%$ sont amurrites, $33.6 \%$ sont accadiens, $9 \%$ sont hurrites, $16 \%$ sont d'origine inconnue et $1 \%$ divers. ${ }^{647}$ On voit donc qu'à l'époque des archives, les Amurrites sont en majorité. Or, à l'époque qui précède Yahdun-Lim, il n'y a pas à Mari de personnes portant des noms amurrites. Dès l'époque présargonique, jusqu'au début de l'époque babylonienne ancienne (début du 19ème siècle avant notre ère), Mari était considérée comme "a provincial outpost of the

\footnotetext{
${ }^{645}$ Voir J.T. Luke, Pastoralism, p. 279.

646 Voir J.-R. Kupper, Nomades, p. XVI.

${ }^{647}$ A Study of Akkadian Personal Names from Mari, University Microfilms International, Ann Arbor - London, 1981, p. 17 et cfr. J.-R. Kupper, Nomades, p. 262; $R L A$ VII/5-6, p. 388.
} 
Babylonian civilization".648 On peut supposer que le commencement de l'apparition des Amurrites dans la zone de Mari est à situer au milieu du 19ème siècle avant notre ère, où, suivant les "Chroniques assyriennes", il faut situer le début de la dynastie à laquelle appartenait Samsi-Addu. C'est l'époque de Sūmu-la-El, le roi de la Babylonie. La liste des rois assyriens débute par le dénombrement des "dix-sept rois qui habitent les tentes".649 Une partie des noms de cette liste ressemble aux noms qui ouvrent la liste généalogique de la dynastie de Hammurabi. ${ }^{650}$ Nous constatons qu'il ne s'agit pas ici de noms propres, mais des éponymes des tribus. Parmi les tribus, nous identifions dans les deux listes le Hanûm, le Didānum (voir ci-dessous) et le Numḩā, et dans la liste babylonienne nous trouvons aussi les tribus Amnanûm et Yahrurûm. D'après les listes généalogiques de la dynastie de Samsi-Addu et de la dynastie de Hammurabi, on constate que l'origine des deux rois est amurrite, comme on pouvait aussi le déduire des noms amurrites de ces rois. Le début de la dynastie de Samsi-Addu est à fixer, comme nous l'avons vu, au milieu du 19 ème siècle avant notre ère. D'où la conclusion que la pénétration des Amurrites dans la Djéziréh a eu lieu au début du 19ème siècle avant notre ère, ou, peut-être mieux encore, au cours du 20 ème siècle avant notre ère. ${ }^{651}$ Cette conclusion s'accorde avec les témoignages sur la pénétration des Amurrites dans la Mésopotamie, que nous trouvons dans les documents de la Babylonie à partir du milieu du troisième millénaire avant notre ère. ${ }^{652} \mathrm{~A}$ côté de certaines personnes nous trouvons la désignation ethnique "Amurrite". ${ }^{653} \mathrm{Le}$ nombre de ces Amurrites croît à partir du règne de Šlgi, le deuxième roi de la

${ }^{648}$ Voir I. Gelb, JCS XV (1961), pp. 34-35 et J.R. Kupper, Nomades, p. 70, n. 3; p. 206, n. 2 et n. 4; p. 262. Cfr. aussi la liste des noms propres dans XIX, pp. 159-162.

${ }^{649}$ Voir I. Gelb, JNES XIII (1954), p. 210, Col. I.10.

650J. Finkelstein, JCS XX (1966), pp. 97-102; D. Charpin et J.-M. Durand, RA LXXX (1986), pp. 159-170 et voir aussi M.12803.I.19-20.

${ }^{651}$ J.-R. Kupper (Nomades, p. 33) fixe leur apparition entre l'époque des "gouverneurs" à Mari pendant la troisième dynastie d'Ur et l'époque de Yahdun-Lim.

${ }^{652}$ Voir J.-R. Kupper, Nomades, p. 150.

${ }^{653}$ Voir J.-R. Kupper, Nomades, pp. 150-151. 
troisième dynastie d'Ur (2094-2047 avant notre ère) et culmine durant le règne du dernier roi de la dynastie, Ibbi-Sîn (2028-2004 avant notre ère). 654

L'examen des noms des gens qualifiés d' "Amurrites" qui apparaissent dans les documents de la troisième dynastie d'Ur montre que $20 \%$ sont des noms sumériens, $14 \%$ accadiens, $9 \%$ accadiens ou amurrites, $40 \%$ amurrites et le reste d'origine inconnue. $655 \mathrm{Il}$ faut aussi signaler que des étrangers qualifiés d' "Amurrites" (dans les documents de Puzriš-Dagān, Isin et Ur) gardent leurs noms amurrites; ${ }^{656}$ par contre parmi les gens "Amurrites" qui sont intégrés dans la société suméro-accadienne (dans les documents de Girsu et Umma), il n'y a presque pas de gens portant des noms amurrites. ${ }^{657}$ Ce phénomène est dû au processus d'assimilation des Amurrites dans la société suméro-accadienne. ${ }^{658}$ A côté de la lente infiltration en Babylonie des individus et des familles isolées des Amurrites, on voit de vraies invasions par de grands groupes d'Amurrites. Pour faire face à ces invasions les rois de la troisième dynastie d'Ur, à partir de Sulgi, ont bâti une muraille fortifiée de $280 \mathrm{~km}$ de longueur, dans la ligne Fallūyah (sur l'Euphrate) - Samarra (sur le Tigre). ${ }^{659}$ La quatrième année de Su-Sîn, le petit-fils de Sulgi (2037-2029 avant notre ère), porte le nom: "Su-Sîn le roi d'Ur a bâti 'la muraille amurrite qui éloigne le Tidnum' ",660 et dans l'inscription royale de sa 9ème année nous lisons: "Quand (Su-Sîn) a bâti 'la muraille amurrite qui éloigne le Tidnum', et a chassé de son pays les forces des Amurrites".661 Dans une lettre que l'un de ses serviteurs lui a écrite, nous lisons: "tu m'as ordonné de construire 'la muraille amurrite qui éloigne le Tidnum' ... parce que les Amurrites ont envahi le pays", 662 et à l'épo-

${ }^{654}$ Voir D.O. Edzard, Die 'Zweite Zwischenzeit' Babyloniens, Wiesbaden 1957, p. 42;

G. Buccellati, The Amorites of the Ur III Period, Naples 1966, pp. 256-267.

${ }^{655} \mathrm{G}$. Buccellati, The Amorites, p. 100.

656 ibid., pp. $254,282,307,344$.

657 ibid., p. 344.

658 ibid., pp. $323,356$.

${ }^{659}$ Voir C. Wilcke, WO V (1969-1970), pp. 1, 9.

${ }^{660}$ Voir D.O. Edzard, Die 'Zweite Zwischenzeit', p. 33.

${ }^{661}$ Voir C. Wilcke, WO V, p. 9.

${ }^{662}$ Voir C. Wilcke, WO V, pp. 7-8, 11. 3-4; H. Limet, RA LXXII (1978), p. 4. 
que du fils de Su-Sîn, Ibbi-Sîn, quand le royaume a commencé à s'ébranler de l'intérieur, ${ }^{663}$ nous lisons dans une lettre qui lui est envoyée par le commandant Išbi-Erra: "Il y a des rumeurs que l'ennemi amurrite a envahi ton pays ... Maintenant tous les Amurrites ont pénétré dans le pays. Ils ont conquis l'une après l'autre les grandes forteresses".664 L'invasion amurrite provoquait l'affaiblissement du royaume d'Ur du point de vue militaire, administratif et surtout économique. Les Amurrites ont pris possession des territoires qui se trouvaient en dehors des villes fortifiées en les isolant l'une après l'autre. Les champs ont été négligés et le prix de l'orge s'élevait sans cesse, jusqu'à 60 fois le prix normal. 665 A la suite de l'effritement du royaume et des invasions des Amurrites, les Elamites ont réussi à infliger une défaite à Ibbi-Sîn, le dernier roi de la troisième dynastie d'Ur. Sur les débris du royaume d'Ur, nous voyons surgir des monarchies amurrites à Larsa, Babylone, Kiš, Marad, Sippar, Kazala, Kisurra, Ešnunna et dans d'autres villes de la région de la Diyāla. ${ }^{666}$ De même, on constate qu'il y a une augmentation considérable du nombre de gens qui portent des noms amurrites - un témoignage des infiltrations grandissantes des Amurrites en Babylonie. Après cela, à l'époque de Hammurabi, le nombre des noms amurrites dans la population diminue, témoignant de l'assimilation des Amurrites à la population accadienne, ${ }^{667}$ mais il faut signaler qu'à l'époque d'Ammi-Ṣaduqa, le roi de Babylonie (1646-1626 avant notre ère), on pouvait encore distinguer entre les deux éléments de la société - les Accadiens et les Amurrites. ${ }^{668}$

Le tableau qui ressort de la Babylonie s'intègre bien avec les données que nous avons fournies sur la situation dans la région de Mari et dans la Haute-Mésopota-

${ }^{663}$ Voir T. Jacobsen, JCS VII (1953), p. 38.

${ }^{664}$ Voir D.O. Edzard, Die 'Zweite Zwischenzeit', p. 44; J.-R. Kupper, Nomades, p. 157; T. Jacobsen, JCS VII, pp. 39-41; I. Gelb, JNES XIII, p. 30; G. Buccellati, The Amorites, p. 91; C. Wilcke, WO V, p. 12.

${ }^{665}$ Voir T. Jacobsen, JCS VII, p. 42.

${ }^{666}$ Voir J.-R. Kupper, Nomades, p. 240; I. Gelb, JNES XIII, p. 30.

667 Voir J.-R. Kupper, Nomades, pp. 169 ss., 197-204, 213-215; I. Gelb, JNES XIII, p. 31.

${ }^{668}$ Voir J.-R. Kupper, Nomades, p. 173; I. Gelb, JNES XIII, p. 32; D. Charpin et J.-M. Durand, RA LXXX, p. 158, n. 83 et cfr. VI.76.20-21. 
mie. Il faut supposer que, parallèlement aux infiltrations et aux invasions massives des Amurrites en Babylonie, ils ont pénétré aussi dans le Djéziréh et ce en plus grand nombre, comme il est prouvé par le fait qu'à Mari la proportion des noms amurrites est plus grande qu'en Babylonie. ${ }^{669}$ De même que les Amurrites ont établi des monarchies en Babylonie, ils ont aussi fondé des monarchies dans le Moyen-Euphrate et en Idamaraṣ et dans les villes de l'ouest - à Karkamiš, Halab, Qatanum et Gublā; à la même époque, ils ont aussi pénétré en Canaan. ${ }^{670}$

On trouve une similitude entre la pénétration des Amurrites en Babylonie à la fin du troisième millénaire et au début du second millénaire avant notre ère et la pénétration de la tribu arabe Sammar en Iraq au 17ème siècle de notre ère. ${ }^{671}$ M.P. von Oppenheim 672 cite G.A. Wallin qui écrit: "individuals and families are almost daily emigrating from this [Djebel Šammar dans la péninsule arabe] to Mesopotamia, where they join their kinsmen and continue their nomadic life along the shores of the Euphrates and Tigris, or gradually settle in the villages". 673 Par la suite M.P. von Oppenheim raconte, à la suite de G.A. Wallin, que dans les années de disette des tribus entières pénétraient dans la Mésopotamie, et il arrivait aussi qu'ils envahissent le pays pour des razzias. Ils sont même parvenus jusqu'à la ville de Bagdad. 674

D'où sont sortis les Amurrites qui ont pénétré dans tout le "Croissant fertile" ? On ne peut pas répondre d'une façon catégorique à cette question, mais les historiens ont insisté sur le fait qu'à l'époque de la dynastie d'Akkad (2334-2154 avant notre ère) et qu'à l'époque de la troisième dynastie d'Ur (2112-2004 avant notre ère) le Mont Bisir était appelé "le Mont d'Amurrum". C'est là où habitait la tribu amurrite Tidānum (ou: Tidnum) - Didānum675 (le Dedân biblique) à l'instar des

\footnotetext{
${ }^{669}$ Voir J.-R. Kupper, Nomades, p. 240.

${ }^{670}$ Voir M.H. Heltzer et Sh. Arbel, The Suteans, pp. 1-10.

${ }^{671}$ Cfr. D.O. Edzard, Die 'Zweite Zwischenzeit', p. 36.

672 Die Beduinen, p. 132.

${ }^{673}$ The Journal of the Royal Geographical Society XXIV (1885), p. 187.

${ }^{674}$ Die Beduinen, pp. 132-133.

${ }^{675}$ Voir D.O. Edzard, Die 'Zweite Zwischenzeit', pp. 31-33; G. Buccellati, The Amorites, p. 244.
} 
Sutéens qui erraient dans la zone du Mont Bisir et dans la steppe syrienne à l'époque des archives. Il semble donc que les Amurrites soient venus de la steppe syrienne. 676

Après leur pénétration dans le pays, on voit que plusieurs tribus se sont séparées et que chaque partie se dirigeait vers une autre région pour s'y installer. Une partie de la tribu Amnanûm s'est installée dans la région de Tuttul, dont le roi portait le titre "roi de Tuttul et du pays d'Amnanûm" (M.2802.III.6-7),677 et une autre partie s'est dirigée vers le sud, vers la Babylonie, et s'est installée dans la région d'Uruk, dont le roi Sîn-kašid (1865-1833 avant notre ère) portait un titre semblable "roi d'Uruk, roi d'Amnanûm".678 Une lettre du temps de Sîn-muballiţ, le roi de Babylone (1812-1793 avant notre ère), nous apprend que son armée était composée de membres de la tribu Amnan-Yahrur et qu'elle a été envoyée chez Anam, le roi d'Uruk; dans la lettre il est précisé en outre qu'une aide semblable avait été envoyée de la Babylonie à Uruk déjà du temps de Sîn-kašid. ${ }^{679}$ Enfin, on trouve le Yahrurum aussi dans la région du Tigre. 680

La conclusion à tirer de notre étude est que les tribus amurrites, qui nous sont connues par les documents de Mari, ont pénétré dans le Moyen-Euphrate au cours du 20 ème siècle avant notre ère. A la suite de leur infiltration dans le pays civilisé, elles ont commencé à pratiquer l'agriculture en plus de l'élevage dont elles s'occupaient auparavant. Dans les documents de Mari, nous constatons simultanément deux étapes de l'assimilation des tribus dans le pays: d'un côté les Amurrites qui sont devenus de vrais sédentaires à l'instar des dynasties de Zimri-Lim et SamsiAddu, et une partie des habitants des villes et villages dont les noms propres révèlent l'origine amurrite, bien qu'il aient été considérés sans doute comme de vrais

${ }^{676}$ Voir J.-R. Kupper, Nomades, p. 241; I. Gelb, JNES XIII, pp. 27, 30; G. Buccellati, The Amorites, pp. 251, 327; D. Charpin et J.-M. Durand, RA LXXX, p. 158.

${ }^{677}$ Il est intéressant de noter qu'à l'époque de la troisième dynastie d'Ur, le gouverneur de Tuttul portait un nom amurrite, voir I. Gelb, JNES XIII, p. 35.

${ }^{678}$ Voir J.-R. Kupper, Nomades, p. 51, n. 1.

${ }^{679}$ Voir A. Falkenstein, Baghdader Mitteilungen II (1963), pp. 56-59.

${ }^{680}$ Voir J.-R. Kupper, Nomades, p. 53. 
Accadiens (VI.76.21), et d'un autre côté une population semi-nomade - "Hanûm" (VI.76.20) - qui garde son exclusivité tribale.

Au cours de notre étude, nous avons constaté plusieurs fois qu'il existe une ressemblance frappante entre le genre de vie des tribus semi-nomades du temps de Mari - Ḩanûm et Bini-Yamina - dans la vallée du Moyen-Euphrate et le genre de vie de la tribu bédouine 'Agêdât. Mais il faut se rappeler que les 'Agêdât n'habitaient pas le Moyen-Euphrate depuis l'antiquité, mais au contraire ont immigré làbas seulement au 17 ème siècle de notre ère, venant de la péninsule arabe. ${ }^{681}$ C'est seulement après leur arrivée sur leur site actuel qu'ils se sont adaptés au genre de vie qu'ils menaient à l'époque de la visite de l'anthropologue français $\mathrm{H}$. Charles. On peut supposer que les Hanéens et les Bini-Yamina ont subi le même processus. D'abord, ils nomadisaient à l'instar de Sutûm, dans la steppe syrienne, et, par leur immigration dans le Moyen-Euphrate ils se sont adaptés au nouvel environnement, ils se sont installés dans des villes et des villages et ils ont mené un genre de vie qui combinait l'agriculture saisonnière avec la transhumance saisonnière. $\mathrm{Par}$ contre, les Sutéens ne se sont pas sédentarisés, mais ils ont continué, durant de nombreuses années après l'époque des archives de Mari, à pratiquer dans la steppe syrienne le genre de vie des tribus nomades moutonnières.

Dans le royaume de Mari, on n'aperçoit jamais une tension quelconque entre le citadin et le villageois qui pratiquait l'agriculture et l'élevage. Au contraire, une harmonie complète régnait entre eux. Le roi lui-même était mêlé à tous les domaines de l'économie, car il possédait des terres et des troupeaux dans chaque district. Il veillait au bien-être de ses sujets agriculteurs en temps de paix et surtout en temps de guerre. La tension dans le royaume n'existait pas au niveau économique entre une ville et un village, mais au niveau politique - une lutte entre le pouvoir central et certaines tribus qui ne voulaient pas se soumettre au pouvoir de l'Etat.

Les documents provenant de la Babylonie nous ont renseignés sur la manière dont les nomades amurrites ont pénétré dans le pays cultivé. Quand le pouvoir central était fort, ils pénétraient individuellement ou en petit nombre de familles. Mais, quand le pouvoir central s'est ébranlé, ils ont profité de l'occasion pour pénétrer en force dans le pays cultivé. Les documents de Mari nous décrivent une situation

${ }^{681} \mathrm{H}$. Charles, Tribus moutonnières, p. 1-2. 
où la première vague d'Amurrites s'est sédentarisée complètement, tandis qu'une partie de la seconde vague - les Sutéens - continue de nomadiser dans la steppe et ne montre aucun désir de se fixer. Une deuxième partie - les Hanéens - continue le genre de vie nomade des bergers, mais en même temps s'installe et commence à pratiquer l'agriculture. Enfin une troisième partie - les Bini-Yamina - se trouve à un stade plus avancé dans le processus de sédentarisation, mais continue de sortir pour les transhumances. Les documents des archives de Mari étalent devant nous un riche tableau des relations entre les autorités centrales, les sédentaires, et les tribus qui se trouvent dans le pays civilisé. Parmi les tribus, on trouve celles qui s'intégraient dans le royaume, sans perdre pour autant leur identité tribale, et celles qui, de temps à autre, ont montré une résistance aux autorités. Le passage des relations pacifiques aux relations belliqueuses pouvait être soudain, et la société sédentaire était alors forcée de s'enfermer dans les murailles des villes-fortifiées et d'abandonner les grands espaces du pays au pouvoir absolu des membres des tribus. Puis avec l'intervention du roi, le calme revenait dans le pays qui continuait à mener sa vie dans la paix et la prospérité. 
Chapitre VIII: L'origine des tribus amurrites

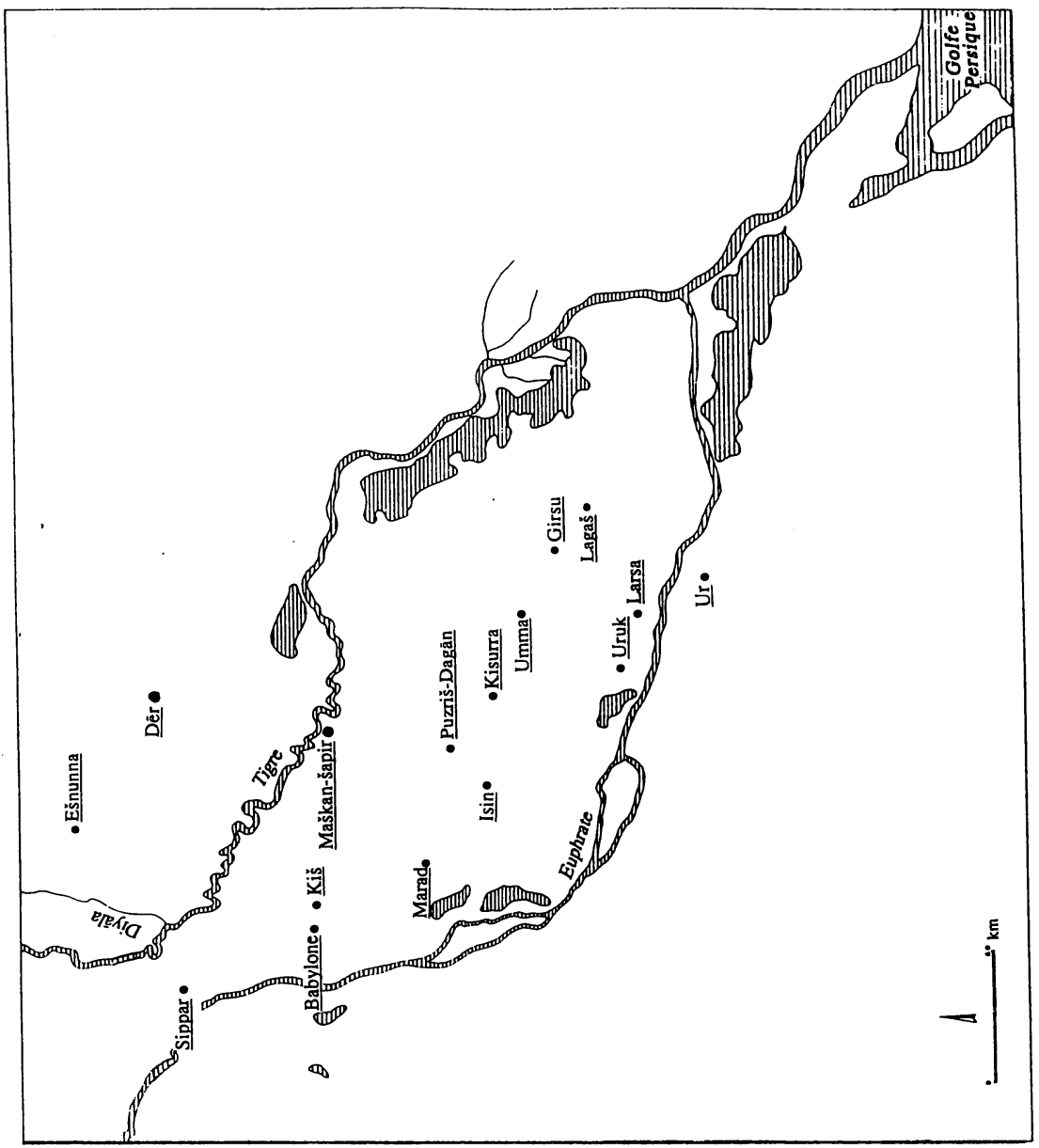

3. La Babylonie 


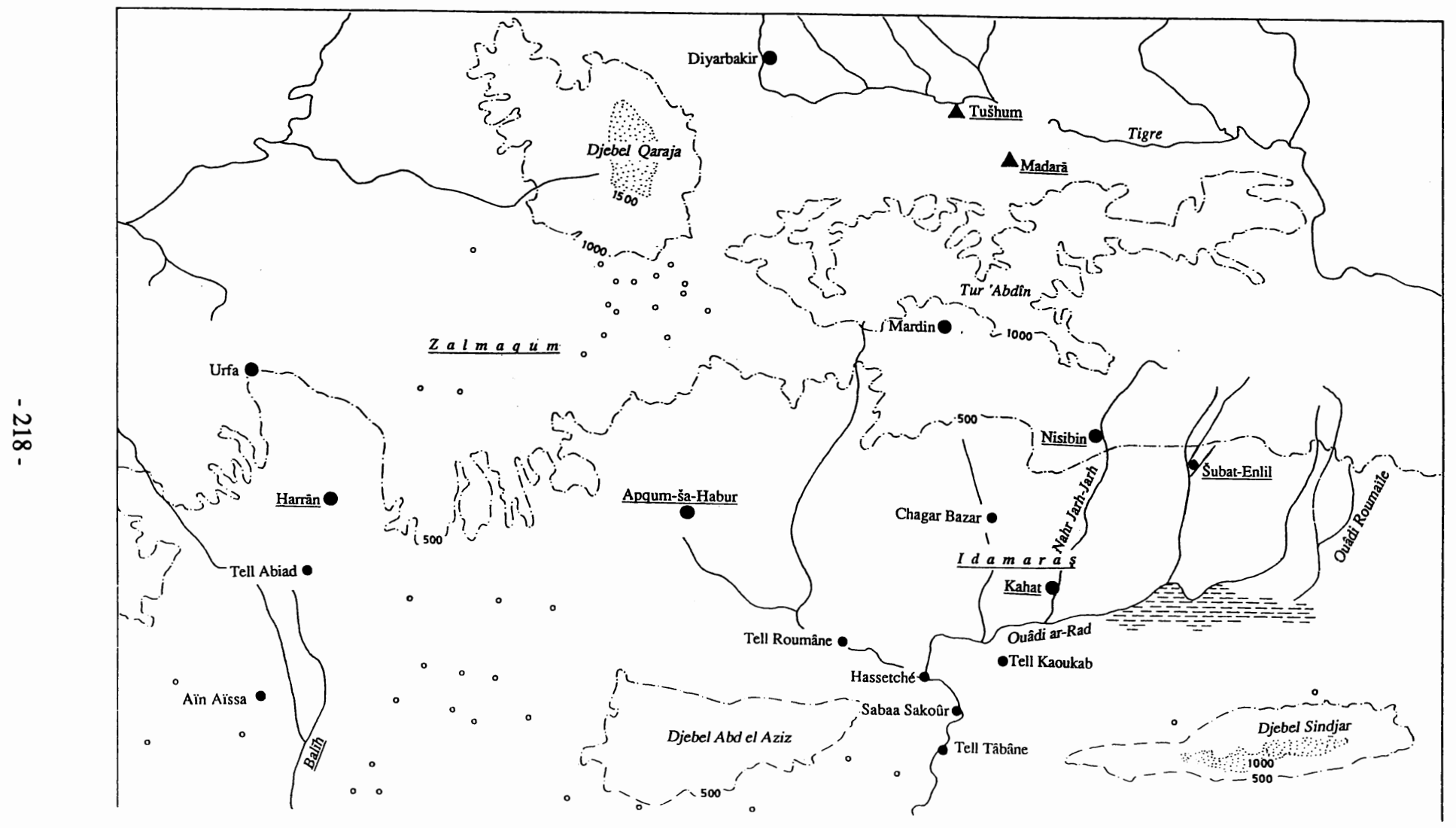




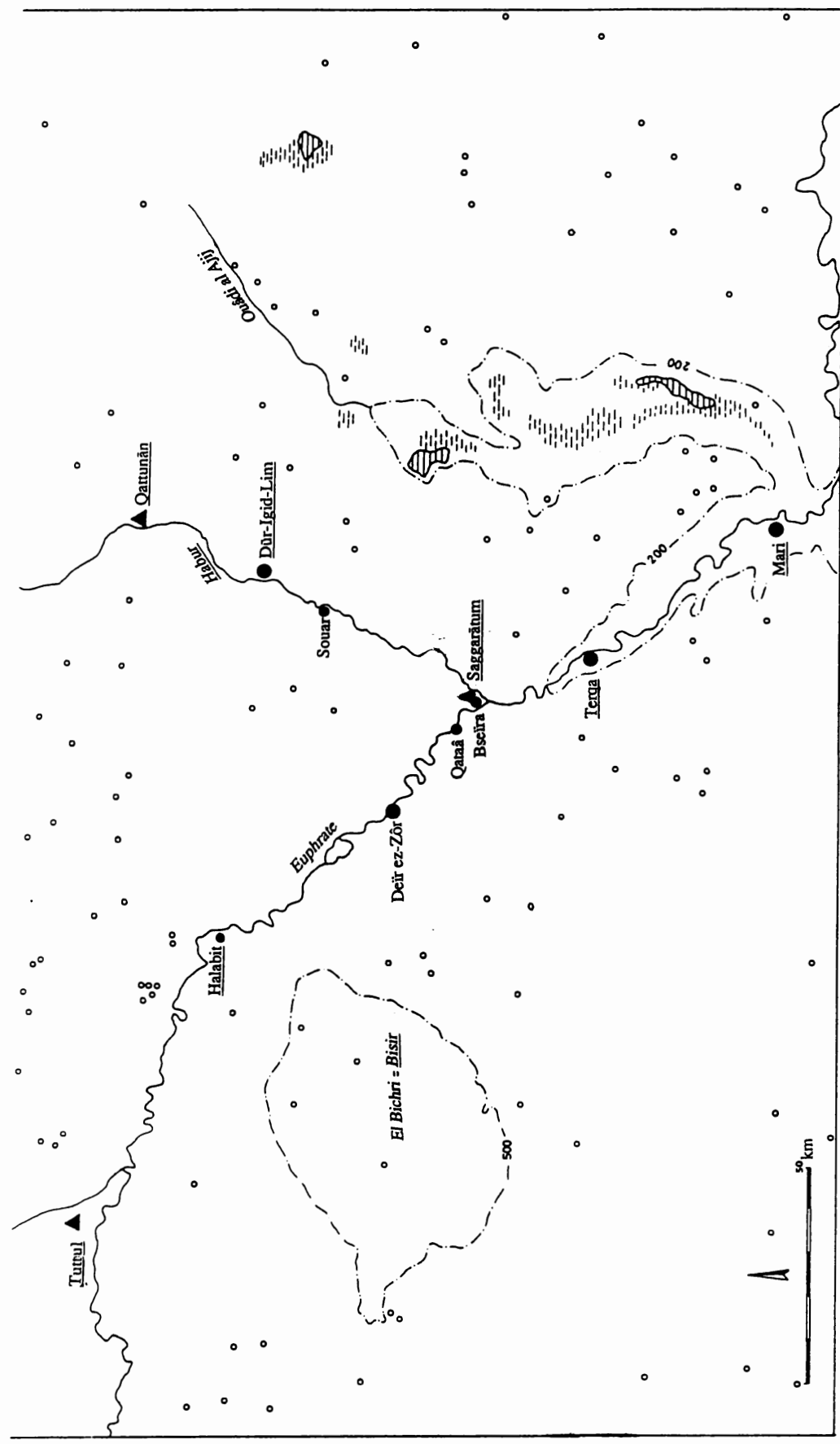

4. Une carte détaillée 


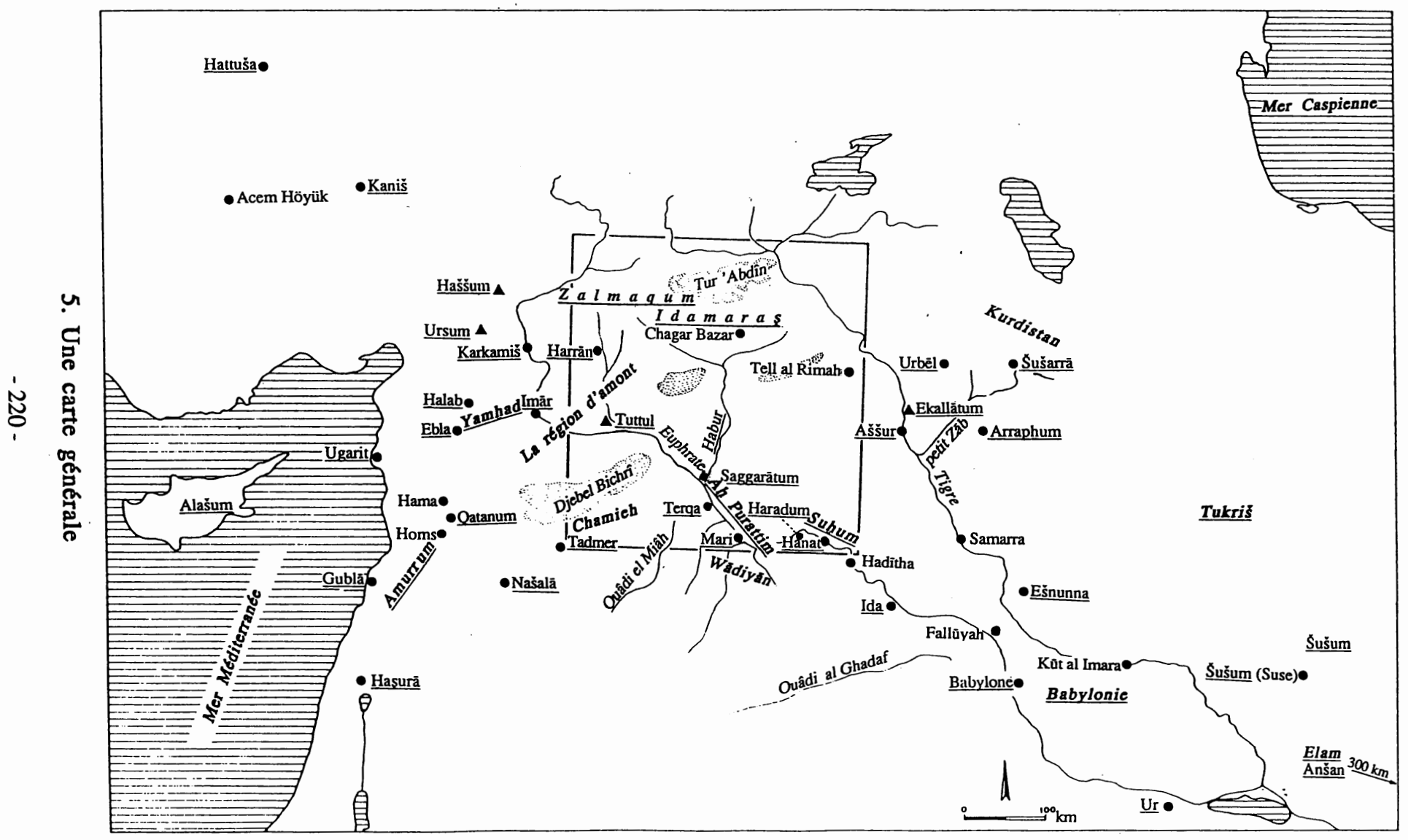

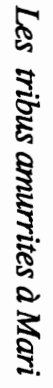




\section{Abréviations}

$A b B$

AfO

AHw

M. Anbar,

Šamši-Addu,

ANET S

AOAT

ARMT

AS

$B A$

$B A R B$

Bi Or

R. Borger,

EAK 1

G. Buccellati,

The Amorites,

BZAW

$C A D$

H. Charles, Tribus moutonnières

CRAIBL

${ }_{\text {CRXe }} \mathrm{RAI}$

J.-M. Durand,

Le système palatial
Altbabylonische Briefe in Umschrift und Übersetzung. Archiv für Orientalforschung.

Akkadisches Handwörterbuch.

L'organisation du royaume de Šamši-Addu $1^{\mathrm{er}}$, Thèse de doctorat, Université de Liège, 1970-1971. Ancient Near Eastern Texts related to the Old Testament, Supplement, J.B. Pritchard (éd.), Princeton 1950. Alter Orient und Altes Testament. Archives royales de Mari, Transcription et Traductions. Assyriological Studies (University of Chicago).

The Biblical Archaeologist.

Bulletin de l'Académie royale de Belgique.

Bibliotheca Orientalis.

Einleitung in die assyrische Königsinschriften, Erster Teil; Das zweite Jahrtausend v. Chr., Handbuch der Orientalistik, Leiden 1964.

The Amorites of the Ur III Period, Naples 1966.

Beihefte zur Zeitschrift für alttestamentliche Wissenschaft. The Assyrian Dictionary of the Oriental Institute of he University of Chicago .

Tribus moutonnières du moyen Euphrate, Damas 1939.

Comptes rendus de l'Académie des inscriptions et BellesLettres (Paris).

Compte rendu de la $X^{e}$ Rencontre Assyriologique Internationale.

Le système palatial en Orient, en Grèce et à Rome,

E. Lévy ( éd.), Strasbourg 1987. 
D.O. Edzard, 'Zweite Zwischenzeit'

M.H. Heltzer et Sh. Arbel, The Suteans

HUCA

HKL

IOS

IRSA

JAOS

JCS

JESHO

JNES

H. Klengel,

Benjaminiten und Ḩanäer

H. Klengel, Zelt und Palast

J.-R. Kupper, Nomades J.T. Luke, Pastoralism

MAM

M.A.R.I.
Die 'Zweite Zwischenzeit' Babyloniens, Wiesbaden 1957.

The Suteans, Naples 1981.

Hebrew Union College Annual.

R. Borger, Handbuch der Keilschriftliteratur, I, Berlin 1967; II, Berlin et New York 1975.

Israel Oriental Studies.

E. Sollberger et J.-R. Kupper, Inscriptions royales sumeriennes et akkadiennes, Paris 1971.

Journal of American Oriental Society.

Journal of Cuneiform Studies.

Journal of Economic and Social History of the Orient. Journal of Near Eastern Studies.

Benjaminiten und Hanäer, Wissenschaftliche Zeitschrift der Humboldt Universität zu Berlin VIII/2-3 (19581959).

Zwischen Zelt und Palast, Vienne 1972.

Les nomades en Mésopotamie au temps des rois de Mari, Paris 1957.

Pastoralism and Politics in the Mari Period: A Reexamination of the Character and Political Significance of the Major West Semitic Tribal Groups of the Middle Euphrates, ca. 1828-1758 B.C., University Microfilm International, Ann Arbor, Michigan 1965.

A. Parrot, Mission archéologique de Mari, I, Paris 1956; II/1, Paris 1958; II/2, Paris 1958; II/3, Paris 1959; III, Paris 1967; IV, Paris 1968.

MARI Annales de Recherches Interdisciplinaires. 
Miscellanea

Babylonica

V. Müller,

Bédouins

N.A.B.U.

no.

$O A$

Or.

$R A$

$R B$

RLA

E. Sachau, Reise

$S E b$

L. Stein,

Schammar-Gerba

UF

$V A B$

VTS
RHPR

Miscellanea Babylonica, Mélanges offerts à Maurice

Birot, J.-M. Durand et J.-R. Kupper (éd.), Paris 1985.

En Syrie avec les Bédouins, Paris 1931.

Nouvelles Assyriologiques Brèves et Utilitaires.

Les textes économiques précédés de "no." sont cités d'après l'article de D. Charpin dans M.A.R.I. III (1984), pp. 83-126.

Oriens Antiquus.

Orientalia.

Revue d'Assyriologie et d'Archéologie orientale.

Revue biblique.

Revue d'histoire et de philosophie religieuses.

Reallexikon der Assyriologie.

Reise in Syrien und Mesopotamien, Leipzig 1883.

Studi Eblaiti.

Die Schammar-Gerba, Beduinen im Übergang vom Nomadismus zur Sesshaftigkeit, Berlin 1967.

Ugarit Forschungen.

Vorderasiatische Bibliothek

Vetus Testamentum, Supplements.

M.P. von Oppenheim, Die Beduinen, Vol. I, Leipzig 1939.

Die Beduinen

WO

$Z A$
Welt des Oriens.

Zeitschrift für Assyriologie. 



\section{Codage des textes cités}

I, II etc. Archives Royales Mari Textes I, ARMT II etc.

I 11, 46 G. Dossin, Correspondance de Samši-Addu, ARMTI, Paris 1950.

I. $39,40,42,43,44$, n. 196

I. 59,50

I. $684,100,110,145,146,162,163,171,180,192$, n. $340,441,597$

I.7 171

I.8 47

I.955

I. 1049

I.13 147, 180

I.17 110, 133, 205

I.18 55

I.19+M.9541 (M.A.R.I. V, pp. 158-159) 49, 180

I. 2046,92

I. 2248,49

I. 2346

I.24+M.5012 (M.A.R.I. IV, p. 312, n. 88) 46, 106, 111, 169, 192

I.27 51

I.29 49

I.37 49, 179

I.39 50

I. $4245,46,48,84,92,162,163,179$, n. 597

I.43 167, 192

I.46 46

I.47 56

I.50 49

I.53+M.7340 (M.A.R.I. IV, pp. 316-317) 50

I.54 46

I.60 49, 85, 204, n. 597

I.62 55

I.67 49

I.69+M.7538 (M.A.R.I. IV, p. 314 et n. 96) 48, 49

I.73 56

I.77 46

I.82 49, 101, 179

I.83 116, 164, 205, n. 514

I.85+A.1195 (M.A.R.I. V, pp. 158-159) 48, 110, 192

I.87 49, 179

I.91+M.5461 (M.A.R.I. V, pp. 178-180) 51, 84, 107, n. 510

I.92 47, 112

I.97 49

I.100 115, 205

I.107 50

I.108 54, n. 196

I.113+A.3905+M.6899+7157 (M.A.R.I. V, pp. 172-174) 54, n. 196

I.118 54

I.119 79, 136, 139, 140

I.121 47

I.123 n. 185

I.128 146, 180, n. 440

I.129 51 
Les tribus amurrites de Mari

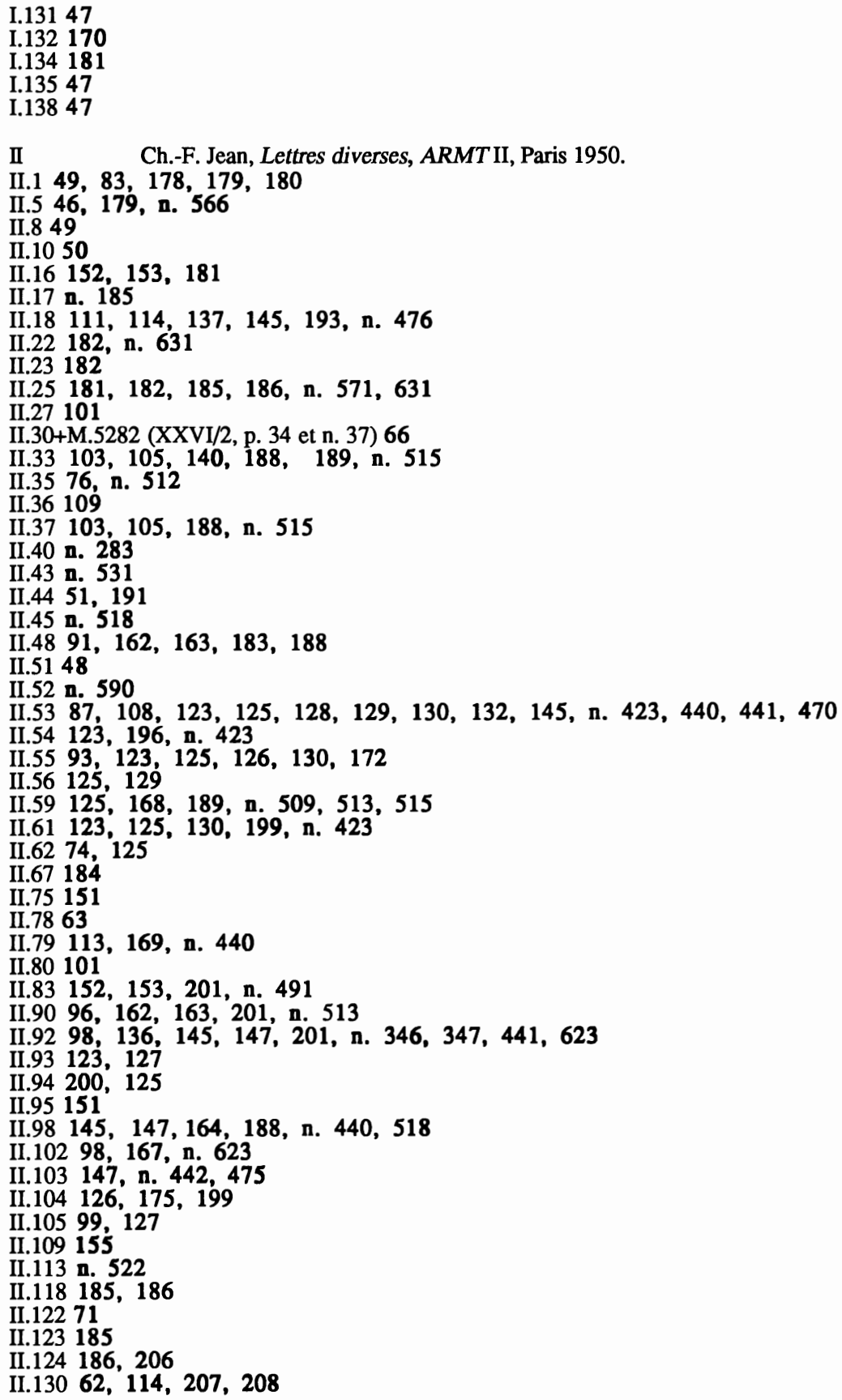


Codage des textes cités

\section{II.135 75}

II.137 109, 121, 130, 155, 198, 202, n. 470

III J.-R. Kupper, Correspondance de Kibri-Dagan, ARMT III, Paris 1950.

III.6 145, 146, 202, n. 441

III.12 97, 164, 207, n. 346, 347, 623

III.14 94, 102, 185,190

III.15 94, 96, 162, 163, n. 509, 513

III.16 96, 197, 201, n. 346, 608

III.17 65, 154

III.18 58

III.19 95

III.20 95

III.21 136, 200, n. 346, 441, 476

III.28 119

III.33 n. 547

III.36 125, 200

III.38 19, 95, 127, 136, 145, 146, 173, 202, n. 441

III.45 127, 200

III.46 92, 190, n. 491

III. 5084,122

III.58 94, 127, 200, n. 346

III.65 76, 151, n. 491

III.70+M.9610 (Mélanges Kupper, pp. 149-151) 127, 128, 148, 174, 202, n. 346

III.71 200

III.73 121, 151, 155

III.83 152

IV G. Dossin, Correspondance de Samši-Addu, ARMTIV, Paris 1951.

IV.1 82, 92, 136, 139, 180, n. 441

IV. 6110,192

IV.7+M.5737 (M.A.R.I. V, pp. 196-197) 146, 147, n. 444, 476, 511, 518

IV.10 46

IV.11 54

IV.16 145, n. 446

IV. 2548,50

IV.27 54

IV. 2850

IV. $29152,153,181$

IV. 3347,112

IV. 3550

IV. $40104,152,153,181$

IV.49 47

IV. 5150

IV.68 151

IV.74 n. $185,348,473,586$

IV.80 104, 181, n. 540

IV.88 n. 185

V G. Dossin, Correspondance de Iasmah-Addu, ARMT V, Paris 1952.

V.2 51, 192, n. 441

V.15 147, 163, n. 440

V.16 48

V.17+A.1882 (M.A.R.I. V, pp. 167-168) 46, 50

V.20 125

V.21 50

V.23 110, 133, 205

V.24 135, 136, 139, 140, 144 
V.26 n. 462

V.27 109, 167

V. 28 n. 462

V.33 n. 185

V.51 50, 102, 179

V.59 n. 185

V.65 73

V.72 146, 180

V.81 82, 98, 164, 172, 180, 193

V.86 172, 180

V, p. 132 , no. 33.9-10 165

VI J.-R. Kupper, Correspondance de Bahdi-Lim, ARMT VI, Paris 1954.

VI.12 151, n. 488, 491

VI. 15133,206

VI. 2358

VI.28 65, 183, 187

VI.30 92, n. $441,477,630$

VI.32 146, n. 441,630

VI.38 n. $441,477,630$

VI.40 136, n. 476

VI.42 86, 165,208, n. 513

VI.44 89, 110, 133

VI.51 206

VI.54 68

VI.57 206

VI.58 n. 643

VI.66 67

VI.68 181 , n. 584

VI.69 187

VI.73 199

VI.76 57, 120, 130, 181, 215, n. 439,668

VI.77 n. 348

VII J. Bottéro, Textes économiques et administratifs, ARMT VII, Paris 1957.

VII.7 50

VII.18 52

VII.19 205

VII.21 52

VII.22 52

VII.31 52

VII.33 52

VII.35 52

VII.43 52

VII.62 52

VII.71 52

VII.75 52

VII.81 52

VII.85 52

VII.87 60

VII.104 64

VII.106 144

VII.110 n. 639

VII. 11764 , n. 454

VII.104 64

VII.106 144

VII.130 151, 154

VII.133 134, n. 638

VII. $140+203137$, n. 440,468

VII. 150 n. 237 
VII. 159 n. 378,620

VII.164 n. 620

VII.165 134, n. $378,620,638$

VII.169 n. 638

VII.201 95, 137, 140, n. 468

VII. 203190, n. 539

VII.210 133, n. $378,620,638$

VII.211 n. 378,620

VII.212 n. 620

VII.213 98, 115, 137, n. 440

VII.215 176, n. $348,440,473,539$

VII.225 91, 95, 99, 137, 138, 206, n. 450, 468

VII.226 91, 93, 126, 199, n. 450,468

VII.227 80, 81, 98, 142, 169, n. 440, 453-458, 464, 539

VII.267 79

VII.277 73

VII.311 135, 139, 141, 144, 151, 153, n. 486

VIII G. Boyer, Textes juridiques, ARMT VIII, Paris 1958.

VIII.6 137

VIII.9+20 205, 206

VIII.11 47, 79, 81, 85, 91, 162, 174, 186

VIII.37 127, 199

VIII.42 52, 127, 130, 199

VIII.61 n. 149

VIII.62 144

VIII.67 99, 126, 199, n. 321

VIII.75 121

VIII.78 190

IX M. Birot, Textes administratifs de la salle 5 du Palais, ARMT IX, Paris 1960.

IX.15 175, 204

IX.23 127, 199

IX.33 (Sc.) n. 407

IX.36 n. 407

IX.43 n. 531

IX.48 208

IX.70 142, n. 457,463

IX.97 n. 425

IX.101 144

IX.102 n. 425

IX.124 n. 235

IX.169 139

IX.241 151, 154

IX.244 79, 88, 170, 206

IX.248 80, 81, 136, 138, 142, n. 440, 441, 453, 454, 457, 464

IX.291 n. 622

IX.298 75

X G. Dossin, Correspondance féminine, ARMT X, Paris 1978.

$X .574,114,131$

X.31 189

X.32 155, n. 495

X.59 204

X.84 71, 113, 137, 144, 169

X.91 188, n. 440

X.107 49

$\mathrm{X} .121$ 155, n. 301

X.140 53, n. 301 
$\mathrm{X} .151126,131$

$\mathrm{X} .15561$

X.156 123, 199

X.157 86, 113, 169, 208

$\mathrm{X} .159123$

$\mathrm{X} .162152$

X.178 50

XI

M.L. Burke, Textes administratifs de la salle 111 du Palais, ARMT XI, Paris

XI.43 n. 324

XI.207 n. 237

XI.250 n. 236

XI.265 n. 237

XII

M. Birot, Textes administratifs de la salle 5 du Palais, $2^{\mathrm{ème}}$ Partie ARMT XII,

XII. 29 n. 429 Paris 1964.

XII.132 127

XIII G. Dossin, J. Bottéro, M. Birot, $M^{m e}$ M. Lurton Burke, J.-R. Kupper et A.

XIII.33 92, 187 Finet, Textes divers, ARMT XIII, Paris 1964.

XIII.36 n. 235

XIII.39 93, 172

XIII.86 126, 176, 202

XIII.102 94, 102, 185, 190, n. 514

XIII.103 94, 102, 185, 190

XIII.105 84

XIII.106 206

XIII.117 151, 154

XIII.123 127

XIII.143 41, 155, n. 495

XIII.144 105, 204

XIII.145 150, 153, n. 301

XIII.146 75

XIII.147 73, n. 301

XIII.148 150, 153, n. 301,491

XIV M. Birot, Lettres de Yaqqim-Addu gouvemeur de Sagarâtum, ARMT XIV, Paris

XIV.8 136, 145 1974

XIV.18 76

XIV.35 175

XIV.36 175

XIV.46 135, 137, 143, 144, n. 474,475

XIV.51 74

XIV.53 191

XIV.55 109, 152

XIV.62 136, 144, 146, n. 475

XIV.64 99, 136, 138, 145, 152, 200, n. 441, 475, 476

XIV.65 145, 152, n. 442,475

XIV.69 68

XIV.75 147, n. 442,475

XIV.78 89

XIV.79 206, n. 299

XIV.80 97, $145,171,188$, n. 440

XIV.81 99, 166, 188

XIV.82 n. 569 
XIV.83 72, 83, 99, 107, 121, 126, 127, 129, 172, 195, 200

XIV.84+M.5343+X (RA LXXX, pp. 176-178) 86, 96, 100, 107, 156, 169, 197

XIV.85 99, 162, 166, 188, n. 510, 513, 628

XIV.86 16i, 175, 197, n. 531

XIV.87 123, 127

XIV.88 108, 132

XIV.89 98, 99,201, n. 608

XIV.92 106, 169, n. 518

XIV.93 n. 423

XIV.103 67

XIV.104+A.472 (XXVI/2, p. 37, n. 59) 67, 154, n. 185

XIV.106 61

XIV.112 190

XIV.114 150, n. 449, 491

XIV.120 104, 189

XIV.121 153, 162, 165, 185, n. 488

XIV.122 64

XIV.124 67

XIV, p. 239, no. 106185

$\mathrm{XV} \quad \mathrm{J}$. Bottéro et A. Finet, Répertoire analytique des tomes I à V, ARMT XV, Paris 1954.

XVI/1 M. Birot et J.-R. Kupper, Répertoire analytique, (2 $2^{\mathrm{e}}$ volume), ARMT XVI/1, Paris 1954.

XVIII O. Rouault, Mukannišum, l'Administration et l'Economie palatiales à Mari,

XVIII.54 136, n. 471 ARMT XVIII, Paris 1977.

XVIII.56 139, n. 471

XVIII.61 n. 639

XVIII.64 n. 639

XVIII, p. 109, S.143, no. 132127

XIX H. Limet, Textes administratifs de l'époque des šakkanakku, ARMT XIX, Paris 1976.

XXI J.-M. Durand, Textes administratifs des salles 134 et 160 du Palais de Mari,

XXI.2 134 ARMT XXI, Paris 1983.

XXI.3 n. 271, 468

XXI.4 134

XXI.5 71

XXI.10 134

XXI.56 93, 95 , n. 468

XXI.59 n. 321

XXI.64 71

XXI.76 190

XXI.83 n. 593

XXI.100 n. 265

XXI.110 n. 236

XXI.128 121, n. 128

XXI.141 71

XXI.142 71

XXI.143 n. 293

XXI.144 n. 293

XXI.145 n. 293

XXI.170 205

XXI.198 136 


\section{XXI.200 56}

XXI.219 123, 127, n. 428

XXI.252 126

XXI.321 n. 619

XXI.333 138, n. 430, 447, 471

XXI.336 134, n. 639

XXI.339 126, n. 324

XXI.348 n. 271

XXI.370 126, 205, n. 422

XXI.375 138, n. 440, 471

XXI.388 152

XXI.412 60, 94

XXII J.-R. Kupper, Documents administratifs de la salle 135 du Palais de Mari, ARMT XXII/1-2, Paris 1983.

XXII.27+65+M.5760 (XXIII, pp. 504-505) 60, 190, 205, n. 616

XXII.40 80, n. 348, 581

XXII.41 80, n. 581

XXII.66 60

XXII.121 174, 202, n. 425

XXII.123 41

XXII.127 73

XXII.140 207

XXII.147 163, n. 595

XXII.150 n. 644

XXII.153+170 137

XXII.160 n. 140

XXII.161-162 41

XXII.167 n. 596, 639

XXII.170 134, 139

XXII.171 163

XXII.203 202

XXII.222 52, 191, 202, 208

XXII.224 177

XXII.227 41

XXII.231 52

XXII.232 62

XXII.248 60

XXII.258 59, 103, 181

XXII.262 173, 208

XXII.264 85

XXII.269 43

XXII.272-273 41

XXII.277-278 41

XXII.290 177

XXII.292 202

XXII.293 147

XXII.303 73

XXII.313 52, n. 296

XXII.326 136, 138, 139, n. 471

XXII.327 n. 596,639

XXII.328 n. 324

XXIII G. Bardet, F. Joannès, B. Lafont, D. Soubeyran et P. Villard, Archives XXIII.12 n. 422 administratives de Mari, ARMT XXIII, Paris 1960.

XXIII.14 202

XXIII.15 127, n. 422

XXIII.16 127, n. 422

XXIII.18 127, n. 422 
XXIII.19 n. 422

XXIII.20 138, 139, n. 440, 471

XXIII.21 139, n. 471

XXIII.42 63

XXIII.57 170

XXIII.69 99, 171, 173, 190, n. 321, 633

XXIII.70 173, 202

XXIII.84 62

XXIII.85 n. 583, 594

XXIII.86 169, n. 594, 622

XXIII.87 81, n. 594

XXIII.91 190

XXIII.216 n. 237

XXIII.222 126, n. 440, 468, 539

XXIII.225 n. 440

XXIII. 235 n. 583, 594

XXIII.236 n. 622

XXIII.237 136, 176, 191

XXIII. 241 n. 620,638

XXIII. 335 п. 412

XXIII.336 n. 412

XXIII.337 133

XXIII.341 n. 539

XXIII.370 59

XXIII.426 173

XXIII.427 n. 321

XXIII.428 $68,85,93,124,128,129$, n. 477,630

XXIII. 429 68, 85, 93,124,128, 129, 201, 202, n. 477, 630

XXIII.433 207

XXIII.435 146, 187, n. 250, 473

XXIII.445 60

XXIII.446 n. 447, 471, 595, 639

XXIII.448 n. 596, 639, 644

XXIII.455 127, n. 422

XXIII.494 n. 593

XXIII.504 104, 151, 154, n. 539

XXIII.514 59

XXIII.540 65, 111, 190, n. 615

XXIII.542 66, 67, 107,126

XXIII.545 68

XXIII.561 191

XXIII.564-567 n. 265

XXIII.569 114

XXIII.570 114

XXIII.592 112, 115, 168, 206

XXIII.593 176, n. 622

XXIII.595 52

XXIII.596 n. 632

XXIII, p. 48194

XXIII, p. 496, n. 113 n. 441

XXIV Ph. Talon, Textes administratifs des salles " $Y$ et $Z$ " du Palais de Mari, ARMT XXIV, Paris 1985.

XXIV.6 144, 171, n. 440

XXIV.23 81, 115, n. 440, 468

XXIV.29 182, n. 468

XXIV.31 126, 169

XXIV.32 n. 446,468

XXIV.53 59, n. 440,453 


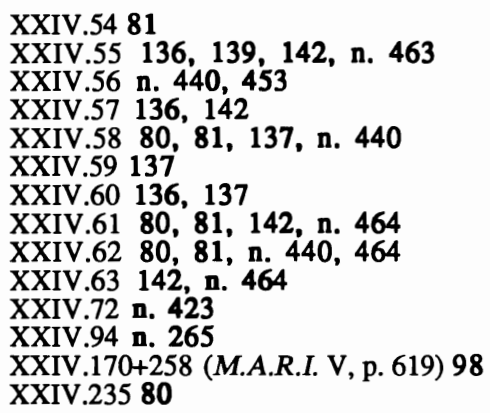

XXV H. Limet, Textes administratifs relatifs aux métaux, ARMT IX, Paris 1986.

XXV.1972

XXV.38+46 107, 126

XXV.48+39+XXI.252 107

XXV.100 191, n. 471

XXV.11350

XXV.114 n. 235

XXV.117 126

XXV.120 (N.A.B.U. 1988/2) 72, n. 293

XXV.130 86, 204

XXV.134 67

XXV.136 107, 126

XXV.143 175

XXV.198 50

XXV.424 59

XXV.593 136, n. 463

XXV.602 180

XXV.616 59

XXV.740 191

XXV.759 n. 464

XXV.760 80, n. 440,464

XXV.783 73

XXVI J.-M. Durand, D. Charpin, F. Joannès, S. Lackenbacher et B. Lafont Archives épistolaires de Mari I/1-2, ARMT XXVI/1-2, Paris 1988.

XXVI.5 136, 141, 144, 176, 204, n. 209

XXVI.6 136, 208, n. 474

XXVI.10 60

XXVI.12 128

XXVI.1698

XXVI.18 208

XXVI.24 108, 109, 128, 145, 153,191, 194, 197, 198, n. 379, 441, 449

XXVI.27 182, 183

XXVI.29 183

XXVI.30 113, 183

XXVI.31 101, 167, 182

XXVI.36 115

XXVI.37 62, 115

XXVI.39 207, n. 354, 441

XXVI.40 103,145, 153,166, 168, 183, n. 233

XXVI.41 91, 208

XXVI.42 n. 596

XXVI.43 n. 575

XXVI.44 146, 157

XXVI.45 156 
XXVI.46 157

XXVI.52 186

XXVI.61 n. 209

XXVI.62 86, 97

XXVI.74 126, 127, 172

XXVI.88 55

XXVI.97 187

XXVI.106 175, 185, 186

XXVI.114 126

XXVI.121 194

XXVI.140 83

XXVI.141 73, 186

XXVI.144 97, 204

XXVI.148 59

XXVI.156 n. 185

XXVI.158 n. 158, 197

XXVI.168 126, 162, 172, 176, 194, n. 342, 414, 419, 420, 509

XXVI.169 126, 176, 194, n. $342,419,420$

XXVI.170 121, 126, 176, 172, 194, n. 342, 414, 420

XXVI.171 126, 172, 176, 194, n. $342,414,420$

XXVI.172 126, 176, 194, n. 342, 414, 419

XXVI.180 162, 165, n. 512, 565

XXVI.181 172

XXVI.185-bis 71

XXVI.189 102

XXVI.199 99, 201, n. 415

XXVI.200 86, 174

XXVI.206 151, 153

XXVI.209 71

XXVI.210 71

XXVI.21271

XXVI.213 58

XXVI.216 176

XXVI.225 116, 207

XXVI.233 122

XXVI.241 137, 148

XXVI.249 150

XXVI.257 184

XXVI.260 n. 530

XXVI. 265 n. 201

XXVI.266 n. 184

XXVI.282 87, 88, 93, 115, 124, 172

XXVI.292 54

XXVI.301 73

XXVI.302 114

XXVI.303 60, 61, 66, 67, 68, 184, n. 206

XXVI.305 67

XXVI.307 67

XXVI.310 68, 74

XXVI.311 68

XXVI.323 67

XXVI.327 66

XXVI.328 66

XXVI.341 71, n. 289

XXVI.342 112

XXVI.345 147

XXVI.346 n. 217

XXVI.347 60, 74

XXVI.352 63, n. 237 
Les tribus amurrites de Mari

XXVI.358 83, 103, 168, 190, n. 391

XXVI.360 n. 234

XXVI.361 n. 249

XXVI.362 63

XXVI.365 n. 243

XXVI.367 66

XXVI.370 66, n. 243

XXVI.372 70, 71

XXVI.373 70

XXVI.376 66

XXVI.377 66, 184

XXVI.378 70

XXVI.381 70

XXVI.383 70

XXVI.384 66, 67, 70, n. 249, 417

XXVI.385 57, 70, 85, 204

XXVI.386 70, 182, 184, n. 579

XXVI.389 112, 189

XXVI.392 67, 69

XXVI.393 151, 152

XXVI.394 112, 113, 189, 207, 208

XXVI.398 56, n. 199

XXVI.404 75, 114, 153, 174, n. 269

XXV1.409 72, 74

XXVI.410 74

XXVI.412 69, 113, 208

XXVI.413 69

XXVI.415 113

XXVI.419 69, 113, n. 283

XXVI.420 161

XXVI.421 114

XXVI.422 168

XXVI.423 62, n. 283

XXVI.425 70, n. 283

XXVI.426 n. 283

XXVI.427 166, n. 283, 531

XXVI.428 71

XXVI.431 71

XXVI.432 71, 113

XXVI.433 71, 75

XXVI.434 71, 114

XXVI.435 71

XXVI.438 152, n. 289

XXVI.439 n. 523

XXVI.440 71

XXVI.440-bis 71, n. 523

XXVI.447 152

XXVI.449 56, 63, n. 570

XXVI.450 136, 140, 146, n. 346, 441

XXVI.451 71

XXVI.456 115

XXVI.462 137, 197

XXVI.463 134, 152

XXVI.468 82, 115

XXVI.470 n. 265

XXVI.471 70, n. 570

XXVI.479 154

XXVI.480 61, 62, 82, 98, 171

XXVI.481 n. 523, 525

XXVI.482 207 
XXVI.483 164, 206

XXVI.491 n. 283

XXVI.496 n. 289

XXVI.498 n. 289, 523

XXVI.499 n. 289,523

XXVI.500 n. 289, 523

XXVI.503 151, n. 218

XXVI.504 n. 218

XXVI.505 72

XXVI.506 72

XXVI.508 112, 146, 147, n. 440

XXVI.510 111

XXVI.511 69, 113

XXVI.513 n. 283

XXVI.519 n. 269

XXVI.520 n. 283

XXVI.521 113, n. 283

XXVI.522 70, 113, n. 283

XXVI.523 n. 283

XXVI.524 168, 170

XXVI.525 n. 283

XXVI.526 70, n. 283

AREC. G. Dossin, "Les archives économiques du Palais de Mari", Syria XX (1939), AREC., p. 104170

pp. 97-113 = Recueil Georges Dossin, Mélanges d'Assyriologie (1934-1959), pp. 133-149, Leuven (s. d.).

AREP. G. Dossin, "Les archives épistolaires du Palais de Mari", Syria XIX (1938),

pp. $105-126=$ Recueil Georges Doss
AREP., p. $108+$ BENJ., p. 991a 105, n. 607

AREP, p. $109\left(^{*}\right) 76,120,161,177$, n. $144,512,515$

AREP., pp. 111-112 123

AREP., p. $112 \mathrm{a} 127,182$

AREP, p. $122 b$ n. 185

AREP., 123a 103

BENJ. G. Dossin, "Benjaminites dans les textes de Mari", Mélanges syriens offerts à Monsieur R. Dussaud, Paris 1939, pp. 981-996 = Recueil Georges Dossin, pp. $150-165$.

BENJ., p. 984d, e 96, 100, 188, n. 614

BENJ., p. 985a 111, 173

BENJ., p. 985 b 173, n. 633

BENJ., p. $986 \mathrm{~b}$ n. 612

BENJ., p. 986c 104

BENJ., p. 986, n. 199,165, n. 510,628

BENJ., p. 986d-987a n. 512

BENJ., p. 987b 197

BENJ., p. 987d 109, 196

BENJ., p. 988a 161, n. 516, 611

BENJ., p. $988 \mathrm{c} 196$

BENJ., p. 989b 102, 197

BENJ., p. 989c 162, 163, 173, n. 614

BENJ., p. 990a 105, 198

BENJ., p. 990b 105, n. 607, 617

BENJ., p. 991a 105, n. 617

BENJ., p. 991b 105, 109

BENJ., p. $991 \mathrm{c}$ n. 516

BENJ., p. 991d 198 


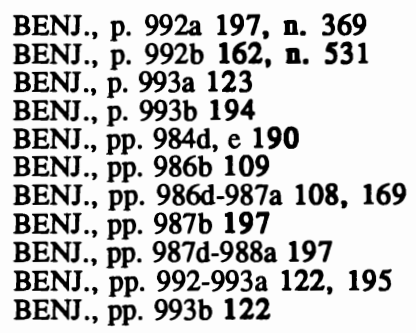

D. Charpin et J.-M. Durand, M.A.R.I. IV, p. 297 , n. 19 n. 156

"Chroniques assyriennes" (M.A.R.I. IV, pp. 227-232) 36, 39, 44-48, 50, 210

EMPR., p. $253 \quad$ G. Dossin, "Légends des empreintes" dans MAM II/3, Paris 1959, pp. 251-257. n.407

"Epopée de Zimri-Lim" (M.A.R.I. IV, pp. 325, 328, 332; XXVI/1, p. 393) 62, n. 192. 476, 588, 602

ETIQ. $\quad$ F. Thureau-Dagin, "Sur des étiquettes de paniers à tablettes provenant de Mari",

Symbolae P. Koschaker, Leiden 1939, pp. 119-120. 32

IAHD. $\quad$ J.-R. Kupper, "L'inscription du 'Disque' de Yahdun-Lim", Kramer Anniversary 150, 406 Volume, Neukirchen-Vluyn 1976, pp. 301-303. 119, 120, 177, n. 147,

IAMH., p. 49 G. Dossin, "Iamḩad et Qatanum", $R A$ XXXVI (1939), pp. 46-54 = Recueil Georges Dossin, pp. 311-319. 161

IASM. (RA XXXIV, pp. 135-139) n. 1

Jean, Ch.-F., EXCE. 1939 "Excerpta de la correspondance de Mari", RES 1938, pp. 128-132. Jean, Ch.-F., EXCE. 1939, no. 702198

LANG Ch.-F. Jean, "La langue des lettres de Mari", RES 1937, pp. 97-112.

LANG., p. 109 168, n. 509, 512

N.AN. $\quad$ G. Dossin, "Les noms d'années et d'éponymes dans les 'Archives de Mari'", N.AN., p. 52, no. 4191 Studia Mariana, A. Parrot (éd.), Leiden 1950, pp. 51-61.

N.AN., p. 52 , no. 6169,191, n. 510

N.AN., p. 55 , no. 593,96

N.AN., p. 52 , no. 7 n. 353

N.AN., p. 55 , no. 6121

N.AN., p. 55, nos. 6-7 193

OBTR St. Dalley, C.B.F. Walker et J.D. Hawkins, The Old Babylonian Tablets from

OBTR 261 Tell al Rimah, British School of Archaeology in Iraq, 1976.

OBTR 361

OBTR 461

OBTR 561

OBTR 9102,168, n. 509,516

OBTR 100148

OBTR 201154

OBTR 244 n. 348

OBTR 245 n. 322

OBTR 254 n. 348

OBTR 263 n. 348

OBTR 267154

OBTR 323 n. 348

OBTR, p. 250 , Sceau 5 n. 407

SH.809 (Sh.T.J. Laessøe, "An Aspect of Assyrian Archaeology", In memoriam Eckhard Unger Beiträge zu Geschichte ..., Baden-Baden 1971, pp. 191-193) 47

SH.811 (ZA XXI, pp. 133-136) n. 174 
SH.818 (Iraq XLVII, p. 93, n. 56) n. 174

SH.827 (Sh.T.J. Laessøe, AS XVI, pp. 189-196) 47, 48, 156

SH.861 (Sh.T.J. Laessøe, The Shemshära Tablets, Copenhague 1959, p. 57) 47, 152

SH.878 (cfr. SH.818 [pp. 48-49]) 48, 49, 152

SH.882 (Iraq XIVII, p. 87, n. 26) 47

SH.888 (Iraq XLVII, pp. 103, 106) 48

SH.913 (Iraq XI VII, p. 100, n. 81) 48

SH.915 (cfr. SH.818 [pp. 45-46]) 48

SH.917 (Iraq XLVII, p. 100, n. 79) 48

SIGN. G. Dossin, "Signaux lumineux au pays de Mari", RA XXXV (1938), pp. 174$186=$ Recueil Georges Dossin, pp. 233-245.

SIGN., p. 17893,96, n. 629

SIGN., p. 181 a 97, n. 629

SIGN., p. 183, n. 291,183

SIGN., p. 184b 103

SIMA. $\quad$ M. Birot, Simahlânê, roi de Kurda, RA LXVI (1972), pp. 131-139.

SIMA., p. 132 n. 429

"Stèle de Samsi-Addu" (RA VII (1910), pp. 151-156) 46, 47

"Stèle de victoire de Zimri-Lim" (M.A.R.I. IV, pp. 320-321) 53, n. 563

Talon, Ph., Miscellanea Babylonica, p. 283 n. 440

TEM III, pp. 16-19, tablette AB M. Birot, Textes économiques de Mari (III), RA XLIX (1955), pp. 16-19 78, 80, n. 581

TEXT. $\quad$ F. Thureau-Dagin, "Textes de Mâri", RA XXXIII (1936), pp. 169-179. 8

TRUP. C. Wilcke, "Truppen von Mari in Kurda", RA LXXIII (1979), pp. 37-50.98, 99, 202,207 , n. 321,591

A.9 (XXVI/1, p. 126; RA LXXX, p. 156, n. 76) 107

A.49 (J. Bottéro, Le problème des Habiru à la $4 \mathbf{4}_{\text {rencotre assyriologique }}$ internationale, Paris 1954, p. 19, no. 20) 104

A.82 (XXVI/1, pp. 184-185) 122, 194

A.215 (XXVI/1, p. 183) 109, 196, 197

A.482 (AREP., p. 117) 57

A.489 (M.A.R.I. IV, p. 186) 53, 193

A.505 (RA LXXXI, p. 135, n. 31) 114, 207, n. 230

A.556 (RA LXXX, p. 154) 86

A.638+ (XXVI/1, p. 16) 76, 161, 170

A.641 (RA LXXX, p. 150, n. 47) 105, 204

A.649 (RA LXXXI, pp. 143-145) 68

A.655 (XXVI/2, p. 39, n. 75$) 68$

A.730 (XXVI/1, p. 385) 129

A.825 (RA LXXIII, p. 151) 137, 141, 144

A.826 (RA LXVI, pp. 116-118) n. 429

A.876 (XXVI/1, p. 184) 83, 169, 191, 197

A.889 ( M.A.R.I. III, pp. 47-48) 53

A.915 (XXVI/1, pp. 160-161) 99, 165

A.954 (XXVI/1, p. 183) 198

A.988 (XXVI/1, p. 306) 112, 189

A.1086 (BENJ., p. 989d) 72, 86, 108, 166, 169, 197, n. 531

A.1089 (XXVI/1, p. 491, n. 42) 161

A.1097 (XXVI/2, p. 306) 113

A.1153 (G. Dossin, "Une opposition familiale", dans La voix de l'opposition en Mésopotamie, Bruxelles 1975, A. Finet [éd.], pp.180-183) 58, 72

A.1176 (XXVI/1, 126, n. 38) 99, 108

A.1188 (RA LXXXII, p. 110) n. 565

A.1191 (XXVI/1, p. 492) 83, 103, 181, 183

A.1208 (BENJ., p. 991b) 85, 197, 204

A.1212 (RA LXXXII, pp. 109-110) 112, 146, 185, 189, 190, n. 370 
A.1251 (J. Bottéro, "L’ordalie en Mésopotamie ancienne, Annali della Scuola Normale superiore de Pisa Classe di lettere e lilosofia, Serie III, Vol. XI, 4, Pisa 1981, pp. 1034-1038) 83, 104, 150, 156, 189

A.1281 (XXVI/1, p. 175) 100, 125, n. 354, 441

A.1288 (M.A.R.I. IV, pp. 227-232) n. 132

A.1610+ (RA LXXXII, pp. 109-110) 104, 112, 113, 140, 146, 185, 189, 190, n. 370

A.1691 (Miscellanea Babylonica , p. 281) n. 440

A.1925 (XXVI/2, pp.313, 242 n. 39 , pp. $470-471) 68$, n. 283

A.1968 (M.A.R.I. IV, p. 28) 40

A.2057 (XXVI/1, pp. 32, 504) 72

A.2094 (UF XVIII, pp. 411-412) 65, 105, 111, 123

A.2231 (M.A.R.I. III, p. 42, no. 1) 44, 96, n. 197

A.2237 (M.A.R.I. V, p. 171) n. 411

A.2238 (Reflets des Deux Fleuves, p. 22b) n. 221

A. 2392 (XXVI/1, p. 545) n. 518

A.2417 (RA LXXXII, pp. 99-100) 75, 150, 153, n. 302

A.2526 (XXVI/1, p. 183) 108, 128, 198

A.2560 (RA LXXX, p. 180) 78, 85, 87, 106, 108, 109, 111

A.2588 (XXVI/1, p. 241) 103

A.2603 (XXVI/2, p. 246) 71

A.2730 (XXVI/2, p. 33) 63, 67, 68, 182, n. 239

A.2741 (CRXVII ${ }^{\mathrm{e}} \mathrm{RII}$, pp. 58-59) 145, 188, n. 440, 618

A.2769 (RA LXVIII, pp. 30-33) 109, 173, 199

A.2801 (RA LXVI, pp. 116-118) 61, 62, 79, 133, 147, 151, 185, 206, n. 429, 475

A.2830 (RA LXVI, pp. 115-116) n. 429

A.2939 (cfr. A. 49, [no. 19]) 106, 207

A.2966+3053 (XXVI/1, p. 133) 65, 183

A.2983 (RA LXVI, pp. 120-121) n. 429

A.3000 (XXVI/2, p. 41) 68, 162

A.3006 (M.A.R.I. IV, p. 297, n. 22) 72

A.3073+ (XXVI/1, pp. 235-236, n. 22) 66

A.3080 (RA LXXX, p. 153 et n. 63) 85

A.3096 (XXVI/2, 189c) n. 431

A.3200 (XXVI/1, p. 115, n. 70) 166

A.3209 (Miscellanea Babylonica, p. 109) 208

A.3274 (Miscellanea Babylonica, pp. 63-64) 193

A.3550 (XXVI/1, p. 125) 98

A.3567 (XXVI/1, p. 184) 153, 183

A.3592 (RA LXXX, p. 163 , n. 108) 89

A.3625 (Miscellanea Babylonica), p. 106120

A.3696 (M.A.R.I. IV, p. 323, n. 132) 53, n. 193

A.3796 (XXVI/1, pp. 396-397) 70, n. 274

A.3821 (CRXVII ${ }^{e} R A I$, pp. 60-61) $47,111,129,132,195$, n. 531

A.3872+ (N.A.B.U. 1988/17[4]) 97

A.3993 (XXVI/1, p. 15) 78, 151

A.4018 (XXVI/1, p. 126) 98

A.4356 (XXVI/2, p. 210 , n. 13 ; p. 127 , n. a) 62

A.4413 (M.A.R.I. V, p. 225) 47

A.4530-bis (XXVI/1, p. 182) $109,167,197$, n. 510

A.4586 (Pouvoirs locaux, p. 61, n. 21) n. 468

A.4634 (RA LXIV, pp. 43-44) 43

B.63 (RA LII, pp. 58-60) 186

B.81 (RA XIII, pp. 70-72) 104, 189, 204

B.308 (RA LX, pp. 19-21) n. 301

B.590 (RA LX, pp. 24-26) 103, 175, 189

M.2 (Syria XXXVII, 221) 151 
M.2802 (Syria XXXII, pp. 4-17) 41, 84, 92, 95, 96, 107, 108, 119, 120, 121, 177. 191,214 , n. $149,348,401,405$

M.5037+ (M.A.R.I. IV, pp. 295-296) 39, 42, n. 129

M.5172 (RA LXXX, p. 154) 79, n. 509

M.5705 (M.A.R.I. V, p. 616) 69, 104

M.6088 (M.A.R.I. IV, p. 265) 52

M.6148 RA LXXX, p. 146, n. 25) 102, 185

M.6210 (XXVI/1, p. 184) 190

M.6435+M.8987 (Mélanges Stève, pp. 111-114) n. 245

M.6874 (XXVI/1, p. 181) 194, 197, n. 418, 491

M.7156+ (Miscellanea Babylonica, pp. 281-282) n. 440

M.7168 (BENJ., p. 994e; M.A.R.I. V, p. 220) 94, 99

M.7407 (XXVI/1, p. 581a) 136

M.7450+ (XXIII, p. 324) n. 321

M.8002 (XXVI/2, p. 14) 54

M.8214 (XXVI/1, p. 112) 63

M.8229+ (Miscellanea Babylonica, pp. 278, 281-282) n. 440

M.8436 (XXVI/1, pp. 77-78) 130, n. 418, 421

M.8512 (M.A.R.I. V, p. 436b) 87

M.8575+ (Miscellanea Babylonica, pp. 278, 281-282) 81, n. 440

M.8795 (M.A.R.I. III, p. 138) n. 343, 549

M.8806 (RA LIV, p. 97$) 66$

M.8990 (XXIII, p. 20) 108, 126

M.9556 (M.A.R.I. III, p. 278) 170

M.9649 (N.A.B.U. 1988/29) 182, 187, n. 408, 445

M.9881 (M.A.R.I. IV, p. 326 ) n. 468

M.9921 (XXVI/1, p. 398) 95, 176

M.10497 (XXVI/1, p. 151, n. 91) n. 236

M.10645 (XXVI/1, p. 151, n. 91$)$ n. 236

M.11167 (UF XVIII, p. 399, n. 83 ) 66

M.11351 (XXIII, p. 476, n. 53) 65, 111, 190, n. 615

M.11357 (XXI, p. 3, n. 7) n. 454, 455

M.11359+M.11593 (Le système palatial, p. 93) 64

M.11395 (RA LXXIII, p. 148, n. 9) n. 471

M.11505 (XXVI/1, p. 143, n. 27) 183

M.11570 (Le système palatial, p. 55, n. 51) n. 472

M.11594 (XXVI/2, p. 131, n. 9) n. 234

M.11634 (M.A.R.I. III, p. 193) n. 422

M.11653 (Miscellanea Babylonica, p. 282) n. 440

M.11837 (Miscellanea Babylonica, p. 281) 81

M.11858 (Miscellanea Babylonica, p. 278) n. 440, 464, 501

M.11940 (Miscellanea Babylonica, p. 282) 136

M.11948 (XXVI/2, p. 209) 69

M.11970 (XXIII, p. 50) 170

M.12074 (Le système palatial, p. 55, n. 53) 127

M.12196+ (Miscellanea Babylonica, pp. 278, 281) n. 440

M.12283+ (Miscellanea Babylonica, p. 278, 281-282) n. 440

M.12620 (Miscellanea Babylonica, p. 281) n. 440

M.12803 (CRXXVI $R A I$, pp. 139-143) n. 650

M.13014 (XXVI/1, p. 468, n. 1) 68

M.13180 (XXIII, p. 304) 59

M.13204 (M.A.R.I. V, p. 232) n. 309

M.13225 (Le systeme palatial, p. 52 ) n. 593

M.13244 (Le système palatial, p. 55, n. 50) n. 440, 593

M.13254 (XXVI/1, p.76) 59

M.13705 (N.A.B.U. 1987/29) 120

M.15093 (Le système palatial, p. 56, n. 10) 137, 142

M.15125+ (Le système palatial, p. 83, n. 136) n. 274

M.15241+ (Le système palatial, p. 83, n. 136) n. 274 
M.15249 (Le système palatial, p. 52, n. 38) n. 593

M.15275+ (Le système palatial, p. 83, n. 136) n. 274

M.18156 (XXVI/2, p. 242, n. 34) 71

M.18229 (UF XVIII, p. 409) n. 621

S.24-1+S.24-2+A.1614b (M.A.R.I. IV, pp. 227-232) n. 132

S.24-2 (ibidem) n. 132

S.24-3 (ibidem) n. 132

S.108, no. 787 (Syria LV, p. 334) 63

S.108, no. 1316 (Syria LV, p. 334 ) 64

S.115, no. 72-2 (Syria L, pp. 4-5.) 48

S.115, no. 72-5 (Syria L, p. 6) 182

S.115, no. $72-15$ (Syria L, p. 9) 74

S.115, no. 72-17 (Syria L, pp. 9-10) 105, 198

S.115, no. 72-38 (Syria L, p. 6) 182

S.115, no. 72-39+72-8 (Mélanges Kupper, pp. 128-131) 59

$S .143$, no. 16 (RA LXXII, p. 187; = M.18156 ?) 71

S.143, no. 132 (XVIII, p. 109) n. 621

T.82 (XXVI/1, p. 380) n. 207

T.178 (XXVI/2, p. 13) n. 635

T.179 (XXVI/2, p. 13) n. 635

T.322+355 (XXVI/1, p. 247) 107

T.338 (XXVI/2, p. 13) n. 635

TH.82.86 (M.A.R.I. III, p. 93, no. 68) 205

TH.82.116 (M.A.R.I. IV, p. 454, n. 3) 52

TH.84.18 (XXVI/1, p. 4, n. 4) 102

no. 46 (M.A.R.I. III, p. 93, no. 68) 52

no. 57 (ibidem, p. 92) 51

no. 59 (ibidem) 51

no. 61 (ibidem) 50

no. 76 (ibidem, p. 94) 50

no. 86 (ibidem, p. 96) 50

no. 97 (ibidem, p. 97 ) 50

no. 98 (ibidem) 52

no. 102 (ibidem, p. 98$) 49$ 


\section{Addendum}

Chapitre I, p. 29: Voir maintenant M.J. Geller, The Lugal of Mari at Ebla and the Sumerian King List, dans C.H. Gordon, G.A. Rendsburg et N.H. Winter (éd.) Eblaitica: Essays on the Ebla Archives and Eblaite Language I, Winona Lake 1987.

Chapitre II, p. 40, Yah̆dun-Lim: Ajouter A.4471.18-23 (M.A.R.I. VI, p. 291).

P. 41, n. 143: Ajouter: D. Charpin, A Contribution to the Geography and History of the Kingdom of Kaḩat, dans M. Wäfler (éd.) Tall al-Hamídiya II, Berne 1990, pp. 68-71.

N. 144: Ajouter: T. 254, T.256, T.358 (D. Charpin,Tall al-Hamidìya II, p. 69, n. 14 ).

P. 42: Une année après la victoire à Nagar et la conquête de la ville, Yahdun-Lim a fait un voyage dans l'Idamaraș. (D. Charpin, Tall al-Hamidìya II, p. 69).

N. 158: Ajouter J.-M. Durand, XXVI/1, p. 325, no. 158, n. a; M.A.R.I. VI, p. 67.

P. 45, L'année Ikūn-pīya: Cfr. J.-M. Durand, M.A.R.I. VI, pp. 276-295.

P. 48: Voir P. Villard, M.A.R.I. VI, p. 581, n. 50.

P. 49: Voir P. Villard, M.A.R.I. VI, pp. 567-584.

P. 56, n. 202: D'un texte inédit on peut déduire que Zimri-Lim était BiniYaminite Rabbéen du côté de sa mère (M.A.R.I. VI, p. 48, n. 48).

P. 57, n. 204: Dans A.3948.5' on trouve la forme [DU]MU Si-im-alki qui d'après J.-M. Durand se réfère à Zimri-Lim (M.A.R.I. VI, p. 54).

P. 59: S.115, no. 72-39+72-8.43-44 (Mélanges Kupper, pp. 128-131).

P. 60, L'année Zimri-Lim 2': Ajouter A.1025.47-50 (M.A.R.I. VI, pp. 337339): Zimri-Lim a pris des villes de Zaziya, le roi des Turukkum, et a razzié ses moutons.

P. 61, L'année Zimri-Lim 3': Ajouter A.1025 (M.A.R.I. VI, pp. 337-339).

P. 67: Ajouter XIII.117+M.5148 (Mélanges Kupper, pp. 156-157).

P. 73: Idamaraş: Le pays entre le Nahr Jarh-Jarh et le Habur supérieur (D. Charpin, Cahiers de N.A.B.U. I [1990], p. 118). 
P. 75: Zimri-Lim est le seigneur et le père des rois de la région au sud du Djebel Sindjar (Bunu-Ištar, Hูatnu-rabi, Šarrum-kīma-kalima, Zimriya et des autres rois) (A.1025.11, 16, 19, 48 [M.A.R.I. VI, pp. 337-339]).

Chapitre III, p. 79: Ajouter A.2796.12-17: les habitants des villes (ālānū) et les habitants de la steppe (hibrum ša nawim) ([M.A.R.I. VI, p. 288]).

P. 86, Bini-Sim'āl: Atamri-El dit aux Imariotes: Les Bini-Sim'āl flambe(?) les Bini-Yamina" (A.3948.5' [M.A.R.I. VI, p. 54]).

P. 88: Un homme venant de chez les Hanéens apporte des renseignements sur le rassemblement des Bini-Yamina (A.2210.5-11 [M.A.R.I. VI, p. 54]).

Chapitre IV, p. 94, B. Bini-Yamina: J.-M. Durand écrit: "Le culte de Dagan de Terqa est loin d'être négligé par les princes benjaminites" (M.A.R.I. VI, p. 53, n. 72). Au dire de Kibri-Dagān, les habitants de quatre villes du district de Terqa (Zurubbān, Hišamta, Hiimmarān et Hुannā) sont les frères des habitants des villes bini-yaminites de son district, III.70+M.9610.8-12 (Mélanges Kupper, pp. 149-151).

N. 320: III.70+.M.9610.9, 25 (Mélanges Kupper, pp. 149-151).

P. 95, c) Raqqum: Samsi-Addu, le roi des Uprapûm séjourne à Raqqum (M.5499 [M.A.R.I. VI, p. 267]).

P. 97: Un habitant de Samānum arrive d'Imār, de chez Bannum (A.3840 [M.A.R.I. VI, p. 53]).

P. 100, B. Bini-Yamina: Dãdi-ḩadum écrit que lorsque la rive gauche était troublée Zimri-Lim a fait prisonniers les Bini-Yamina (A.3185.6-7 [M.A.R.I. VI, p. 48]). Les Bini-Yamina sortent de leurs villes et leur pays (probablement du district de Terqa ou Saggarātum) et montent vers la région d'amont (A.3960 [M.A.R.I. VI, pp. 50-51]). On voit en outre que les Bini-Yamina possèdent des villes et le pays entre les villes (1. 3').

P. 102: 6. Idamaraş: Dans une lettre nous lisons que: "Une paix existe entre les Ḩanéens et Idamaraş. Il paissent (ire'i) jusqu'à (Mont) Zarā" M.9623.13-14 (D. Charpin, Tall al-Hamìdìy II, p. 79).

P. 103: c) Apum: Un Ḩanéen apporte à Ibal-El des nouvelles concernant le sort de Zūzu, le roi du pays d'Apum, A.350+A.616.10-12 (D. Charpin, Cahiers de N.A.B.U. I (1990), pp. 120-121). 
d) Kahahat: Ibal-El arrive avec les Hanéens à Kabittum qui se trouve dans la région de Kahat (M.9175 [D. Charpin, Tall al-Hamīdiya II, pp. 76-77]).

P. 106, b) Subat-Samaš: Sîn-tiri promet à Yasmah-Addu un contingent comprenant aussi des Hanéens (A.4259.5' [M.A.R.I. VI, p. 570]).

P. 107: a) Abattum: Sur Dādi-ḩadun, le roi d'Abattum, voir M.A.R.I. VI, pp. 42-48. Zimrān détient la ville de Yakaltum près de Tuttul (A.2721 [M.A.R.I. VI, p. 58]).

P. 109: Les Bini-Yamina menacent de conquérir Tuttul et d'agresser Imar (A.3960.2'-12' [M.A.R.I. VI, pp. 50-51]). Les Bini-Sim'āl sont attestés probablement dans la région d'Imār (A.3948.5' [M.A.R.I. VI, p. 54]).

- Ajouter A.546 (M.A.R.I. VI, pp. 60-61). Les rois de Bini-Yamina sont chassés d'Imār où ils sont réfugiés (A.3347 [M.A.R.I. VI, pp. 64-65]).

P. 110, n. 356: Pour epēšum dans le sens de "fortifier" voir J.-M. Durand M.A.R.I. VI, p. 46, n. 38.

P. 115, a) Yabliya: Hammānum, le gouverneur de Yabliya informe Yasmah̆Addu à propos des Sutéens qui ont volé des moutons (M.11009+11010 [M.A.R.I. VI, pp. 263-264]).

Chapitre V, p. 122: Ajouter A.3347 (M.A.R.I. VI, pp. 64-65).

P. 125: Samsi-Addu demeure depuis trois jours à Raqqum et s'intéresse à un édit promulgué par Zimri-Lim concernant les Uprapûm (M.5499 [M.A.R.I. VI, p. 267]). Aplahanda, le roi de Karkamiš, demande à Dādi-hadun des renseignements sur la rive gauche de l'Ah-Purattim (entre Saggarātum et Mari) (A.3185.5-10 [M.A.R.I. VI, p. 48]).

P. 126: Dādi-hadun possède des bateaux (M.13096.9 [M.A.R.I. VI, p. 46]); il loue des ouvriers d'Imār pour fortifier sa capitale Abattum (1. 10). Dādi-hadun siège avec l'assemblée d'Imār (A.623+.13-14 [M.A.R.I. VI, p. 55]).

P. 127: Dādi-ḩadun va chez Aplahanda le roi de Karkamiš (A.3185.5-10 [M.A.R.I. VI, p. 48]). Dādi-hadun était l'oncle maternel de Zimri-Lim (M.A.R.I. VI, p. 48, n. 48). Un devin qui se trouve à Imār fait des présages concernant Yasmah-Addu (XXVI.112.7-10).

- III.70+M.9610.23-24 (Mélanges Kupper, pp. 149-151).

N. 422: Ajouter XXII.203+208+XXV.729.IV.43-49 (Mélanges Kupper, pp. 160-177). 
N. 423: Șūra-ḩammu était en visite à Mari durant le Vème mois de l'année ZL 4' et le IXème mois de la même année (cfr. J.-M. Durand Mélanges Kupper, p. 171, n. 41).

P. 129: Les sugāgū et les centeniers des villes bini-yaminites du district de Terqa refusant de faire des habits pour le Palais de Mari, Kibri-Dagān conseille à ZimriLim de s'adresser aux rois bini-yaminites qui se trouvent chez lui (III.70+M.9610 [Mélanges Kupper, pp. 149-151]).

N. 425: Ajouter XXII.127; M.11200 (Mélanges Kupper, pp. 152-153).

P. 132: A Abattum régnait une dynastie bini-yaminite de la tribu Rabbûm: Ayalum au temps de Yah̆dun-Lim et son fils Dădi-hadun au temps de Zimri-Lim (M.A.R.I. VI, p. 48).

P. 135, n. 436: Ajouter: I. Nakata, JANES XIX (1989), pp. 113-118.

P. 136, n. 440: Ajouter: M.9175 (D. Charpin, Tall al-Hamidiya II, pp. 76-77).

N. 441: VI.38+M.5003.6-7, 15, 23 (Mélanges Kupper, pp. 154-155).

P. 144, n. 475: Ajouter III.70+M.9610.8 (Mélanges Kupper, p. 150): à côté des sugăgū des villes bini-yaminites, on trouve des centeniers. ${ }^{1}$

P. 146: Supprimer la référence à XXIII.435.

Pp. 151, 154: XIII.117+M.5148.41-42 (Mélanges Kupper, pp. 156-157).

Chapitre VI, p. 161, n. 512: Ajouter A.3344.13 (M.A.R.I. VI, p. 629).

P. 162, n. 516: Ajouter M.6669.10-11 (M.A.R.I. VI, pp. 271-272).

P. 165: Un nawûm de Zimri-Lim paissant dans la région au sud de Djebel Sindjar est mentionné dans une lettre de Ibal-El, A.350+A.616.36 (D. Charpin, Cahiers de N.A.B.U. I [1990], pp. 120-121).

P. 166: Nous lisons que pour donner du sel-rouge à leur ovin (M.A.R.I. VI, p. 634): "10 parcs à moutons (des Hanéens) sont descendus et ils campent en amont et en aval de la ville, sur les bords du Habur" (A.3344.9-13, et cfr. 11. 17-19 [M.A.R.I. VI, pp. 629-631]).

P. 167: 1.43.10'-12' devenu 11. 36-37 doit être réinterprété à la lumière de la nouvelle collation (M.A.R.I. VI, pp. 568).

\footnotetext{
${ }^{1}$ J.-M. Durand Mélanges Kupper, p. 151, n. b) traduit laputtûm dans ce context par "chef de travaux".
} 
P. 168: Le pâturage de Hanéens en Idamaraș figure aussi dans la lettre M.9623.13-14 (D. Charpin, Tall al-Hamidiya II, p. 79).

P. 170: Sur le miksum voir aussi A.229 et A.4671 (M.A.R.I. VI, p. 81).

Chapitre VII, p. 179, n. 565: D'après J.-M. Durand (M.A.R.I. VI, p. 56, n. 92) Gaššum se trouve dans l'Idamaraș nord ouest.

P. 182: Pour l'année ZL 9' voir aussi XXV.815; A.486+ ( F. Joannès, N.A.B.U. 1989/108; RA LXXXIII [1989], pp. 145-149). Dans cinq lettres de l'année ZL 2' (XXVI.25-30) on parle toujours de l'armée (șābum), ${ }^{2}$ qui est, pensons-nous, composée de gens d'Ah-Purattim; ${ }^{3}$ d'autre part, Asqudum et Halihadun veulent recruter aussi des Hanéens. ${ }^{4}$

P. 183: Ajouter A.4259.6' (M.A.R.I. VI, p. 570). Des Ḩanéens qualifiés comme "troupe d'élite" participent dans l'armée de Zimri-Lim à sa guerre contre Ešnunna (A.1025.6, 69, 71, 73, 80 [M.A.R.I. VI, pp. 337-339]).

- Ibal-El écrit à Zimri-Lim: "Mon seigneur sait que les Hुanéens [entendent mes] instru[ctions]" A.350+A.616.4 (D. Charpin, Cahiers de N.A.B.U. I (1990), pp. $120-121)^{5}$

P. 187: Dans XIII.435 il ne s'agit pas de 3 sugāgū, mais de 3 šāpirū șābim. D'autre part, d'après la lettre A.486+.40-67 et le document XXV.815.Rev.3'-18' (F. Joannès, RA LXXXIII [1989], pp. 145-147) nous constatons que l'unité du "grand-des Ammurites" Baḥdi-Addu comptait 804 soldats, 2 šãpirū șābim, 10 centeniers, 20 cinquanteniers, un scribe et un héraut.

P. 188, n. 589: Notre objection à l'avis de J.T. Luke et H. Klengel doit être rejetée, car dans une lettre on constate qu'un lien existe entre la paix entre l'Idamaraș et les Huanéens et leur pâturage, M.9623.13-14 (D. Charpin, Tall alHamidìya II, p. 79).

\footnotetext{
${ }^{2}$ XXVI.25.5, 26.6, 8, 13, 16; 27.10, 38, 43; 28.7, 16, 21; 29.8', 12', 17', 19'; 30.10.

${ }^{3} \mathrm{XXVI} / 1$, p. 156, n. a. Cela explique l'intérêt exprimé par Asqudum et Hali-Hadun pour "la moisson d'Ah-Purattim" XXVI.31.36-37.
}

${ }^{4}$ XXVI.27.4, 7, 8, 13, 15, 37; 29.5; 30.6, 8.

${ }^{5}$ En restituant ša-a[p-ti-ya et le verbe šemûm], II.55.34; 138.12-13. D. Charpin restitue ša-a[p$r a-k u]$ "que je commande". 
P. 189: Les errances des Hanéens en Idamaraș sont décrites par Ibal-El comme suit: "Tout comme un marchand qui va e[ntre] (un pays) en guerre et (un pays) en pai[x], les Ḩanéens vo[nt] à pied[s(?) entre] (un pays) en guerre et (un pays) en paix et apprennent au cours de leurs errances ce dont parle le pays" A.350+A.616.5-8 (D. Charpin, Cahiers de N.A.B.U. I [1990], pp. 120-121). Le merhum conduit 1000 ou 2000 Hanéens (A.1025.80-81 [M.A.R.I. VI, pp. 337339]).

P. 192: Voir ci-dessus à propos de p. 163 (I.43).

P. 194: Les rois Bini-Yaminites se sont réfugiés aussi à Imār (A.2237 [M.A.R.I. VI, p. 54]). Voir aussi A.4490 (Miscellanea Eblaitica II, p. 31).

P. 195: Dādi-ḩadun en collaboration avec Imār a délaissé la partie des BiniYamina pour s'associer à la partie de Sumhu-rabi, le gouverneur de Tuttul (A.623+.15-17 [M.A.R.I. VI, p. 55]).

P. 196, n. 608: Ajouter A.546 (M.A.R.I. VI, pp. 60-61).

P. 197: Voir aussi A.402 (Miscellanea Eblaitica II, pp. 32-33).

- Sammetar est renseigné sur un rassemblement de 1000 Bini-Yamina disposés à combattre (A.2210.5-11 [M.A.R.I. VI, p. 54]).

P. 202: XXII.203+208+XXV.729.IV.43-49 (Mélanges Kupper, pp. 160-177).

P. 205, B. L'époque de Yasmah-Addu: Hammānum, le gouverneur de Yabliya, écrit à Yasmah̆-Addu: "Au sujet des Sutéens, jamais les Sutéens n'avaient volé des moutons. Or à présent ils viennent de voler des moutons" (M.11009+11010.4-8 [M.A.R.I. VI, pp. 263-264]).

Codage, P. 237: AREP., p. 109: A.1098 (D. Charpin,Tall al-Hamídìya II, p. 70). 


\section{ORBIS BIBLICUS ET ORIENTALIS}

Bd. 1 OTTO RICKENBACHER: Weisheitsperikopen bei Ben Sira. X-214-15* Seiten. 1973. Vergriffen.

Bd. 2 FRANZ SCHNIDER: Jesus der Prophet. 298 Seiten. 1973. Vergriffen.

Bd. 3 PAUL ZINGG: Das Wachsen der Kirche. Beiträge zur Frage der lukanischen Redaktion und Theologie. 345 Seiten. 1974. Vergriffen.

Bd. 4 KARL JAROS: Die Stellung des Elobisten zur kanaanäischen Religion. 294 Seiten, 12 Abbildungen. 1982. 2. verbesserte und überarbeitete Auflage.

Bd. 5 OTHMAR KEEL: Wirkmäcbtige Siegeszeichen im Alten Testament. Ikonographische Studien zu Jos 8,18-26; Ex 17, 8-13; 2 Kön 13, 14-19 und 1 Kön 22, 11. 232 Seiten, 78 Abbildungen. 1974. Vergriffen.

Bd. 6 VITUS HUONDER: Israel Sobn Gottes. Zur Deutung eines alttestamentlichen Themas in der jüdischen Exegese des Mittelalters. 231 Seiten. 1975.

Bd. 7 RAINER SCHMITT: Exodus und Passa. Ibr Zusammenhang im Alten Testament. 124 Seiten. 1982. 2. neubearbeitete Auflage.

Bd. 8 ADRIAN SCHENKER: Hexaplarische Psalmenbrucbstücke. Die hexaplarischen Psalmenfragmente der Handschriften Vaticanus graecus 752 und Canonicianus graecus 62. Einleitung, Ausgabe, Erläuterung. XXVIII-446 Seiten. 1975.

Bd. 9 BEAT ZUBER: Vier Studien $z u$ den Ursprüngen Israels. Die Sinaifrage und Probleme der Volks- und Traditionsbildung. 152 Seiten. 1976. Vergriffen.

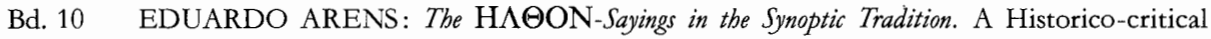
Investigation. 370 Seiten. 1976.

Bd. 11 KARL JAROS: Sichem. Eine archäologische und religionsgeschichtliche Studie, mit besonderer Berücksichtigung von Jos 24. 280 Seiten, 193 Abbildungen. 1976.

Bd. 11a KARL JAROS/BRIGITTE DECKERT: Studien zur Sichem-Area. 81 Seiten, 23 Abbildungen. 1977.

Bd. 12 WALTER BÜHLMANN: Vom recbten Reden und Scbweigen. Studien zu Proverbien 10-31. 371 Seiten. 1976. Vergriffen.

Bd. 13 IVO MEYER: Jeremia und die falschen Propheten. 155 Seiten. 1977. Vergriffen.

Bd. 14 OTHMAR KEEL: Vögel als Boten. Studien zu Ps 68, 12-14, Gen 8, 6-12, Koh 10, 20 und dem Aussenden von Botenvögeln in Ägypten. - Mit einem Beitrag von Urs Winter zu Ps 56, 1 und zur Ikonographie der Göttin mit der Taube. 164 Seiten, 44 Abbildungen. 1977. Vergriffen.

Bd. 15 MARIE-LOUISE GUBLER: Die frühesten Deutungen des Todes Jesu. Eine motivgeschichtliche Darstellung aufgrund der neueren exegetischen Forschung. XVI-424 Seiten. 1977. Vergriffen.

Bd. 16 JEAN ZUMSTEIN: La condition du croyant dans l'Evangile selon Matthieu. 467 pages. 1977. Epuisé.

Bd. 17 FRANZ SCHNIDER: Die verlorenen Söhne. Strukturanalytische und historisch-kritische Untersuchungen zu Lk 15. 105 Seiten. 1977.

Bd. 18 HEINRICH VALENTIN: Aaron. Eine Studie zur vor-priesterschriftlichen Aaron-Überlieferung. VIII-441 Seiten. 1978. 
Bd. 19 MASSÉO CALOZ: Etude sur la LXX origénienne du Psautier. Les relations entre les leçons des Psaumes du Manuscrit Coislin 44, les Fragments des Hexaples et le texte du Psautier Gallican. 480 pages. 1978.

Bd. 20 RAPHAEL GIVEON: The Impact of Egypt on Canaan. Iconographical and Related Studies, 156 Seiten, 73 Abbildungen. 1978.

Bd. 21 DOMINIQUE BARTHÉLEMY: Etudes d'bistoire du texte de l'Ancien Testament. XXV419 pages. 1978. Vergriffen.

Bd. 22/1 CESLAS SPICQ: Notes de Lexicographie néo-testamentaire. Tome I: p. 1-524. 1978. Epuisé.

Bd. 22/2 CESLAS SPICQ: Notes de Lexicographie néo-testamentaire. Tome II : p. 525-980. 1978. Epuisé.

Bd. 22/3 CESLAS SPICQ: Notes de Lexicographie néo-testamentaire. Supplément. 698 pages. 1982.

Bd. 23 BRIAN M. NOLAN: The Royal Son of God. The Christology of Matthew 1-2 in the Setting of the Gospel. 282 Seiten. 1979. Out of print.

Bd. 24 KLAUS KIESOW: Exodustexte im Jesajabuch. Literarkritische und motivgeschichtliche Analysen. 221 Seiten. 1979. Vergriffen.

Bd. 25/1 MICHAEL LATTKE: Die Oden Salomos in ibrer Bedeutung für Neues Testament und Gnosis. Band I. Ausführliche Handschriftenbeschreibung. Edition mit deutscher Parallel-Übersetzung. Hermeneutischer Anhang zur gnostischen Interpretation der Oden Salomos in der Pistis Sophia. XI-237 Seiten. 1979.

Bd. 25/1a MICHAEL LATTKE: Die Oden Salomos in ibrer Bedeutung für Neues Testament und Gnosis. Band Ia. Der syrische Text der Edition in Estrangela Faksimile des griechischen Papyrus Bodmer XI. 68 Seiten. 1980.

Bd. 25/2 MICHAEL LATTKE: Die Oden Salomos in ibrer Bedeutung für Neues Testament und Gnosis. Band II. Vollständige Wortkonkordanz zur handschriftlichen, griechischen, koptischen, lateinischen und syrischen Überlieferung der Oden Salomos. Mit einem Faksimile des Kodex N. XVI-201 Seiten. 1979.

Bd. 25/3 MICHAEL LATTKE: Die Oden Salomos in ibrer Bedeutung für Neues Testament und Gnosis. Band III. XXXIV-478 Seiten. 1986.

Bd. 26. MAX KÜCHLER: Frübjüdische Weisheitstraditionen. Zum Fortgang weisheitlichen Denkens im Bereich des frühjüdischen Jahweglaubens. 703 Seiten. 1979. Vergriffen.

Bd. 27 JOSEF M. OESCH: Petucha und Setuma. Untersuchungen zu einer überlieferten Gliederung im hebräischen Text des Alten Testaments. XX-392-37* Seiten. 1979.

Bd. 28 ERIK HORNUNG/OTHMAR KEEL (Herausgeber): Studien zu altägyptischen Lebenslebren. 394 Seiten. 1979.

Bd. 29 HERMANN ALEXANDER SCHLÖGL: Der Gott Tatenen. Nach Texten und Bildern des Neuen Reiches. 216 Seiten, 14 Abbildungen. 1980.

Bd. 30 JOHANN JAKOB STAMM: Beiträge zur Hebräischen und Altorientalischen Namenkunde. XVI264 Seiten. 1980.

Bd. 31 HELMUT UTZSCHNEIDER: Hosea - Prophet vor dem Ende. Zum Verhältnis von Geschichte und Institution in der alttestamentlichen Prophetie. 260 Seiten. 1980.

Bd. 32 PETER WEIMAR: Die Berufung des Mose. Literaturwissenschaftliche Analyse von Exodus 2, 23-5, 5. 402 Seiten. 1980. 
Bd. 33 OTHMAR KEEL: Das Böcklein in der Milch seiner Mutter und Verwandtes. Im Lichte eines altorientalischen Bildmotivs. 163 Seiten, 141 Abbildungen. 1980.

Bd. 34 PIERRE AUFFRET: Hymnes d'Egypte et d'Israël. Etudes de structures littéraires. 316 pages, 1 illustration. 1981.

Bd. 35 ARIE VAN DER KOOIJ: Die alten Textzeugen des Jesajabuches. Ein Beitrag zur Textgeschichte des Alten Testaments. 388 Seiten. 1981.

Bd. 36 CARMEL McCARTHY: The Tiqqune Sopherim and Other Theological Corrections in the Masoretic Text of the Old Testament. 280 Seiten. 1981.

Bd. 37 BARBARA L. BEGELSBACHER-FISCHER: Untersucbungen zur Göttervelt des Alten Reiches im Spiegel der Privatgräber der IV. und V. Dynastie. 336 Seiten. 1981.

Bd. 38 MÉLANGES DOMINIQUE BARTHÉLEMY. Etudes bibliques offertes à l'occasion de son $60^{e}$ anniversaire. Edités par Pierre Casetti, Othmar Keel et Adrian Schenker.

724 pages, 31 illustrations. 1981.

Bd. 39 ANDRÉ LEMAIRE: Les écoles et la formation de la Bible dans l'ancien Israël. 142 pages, 14 illustrations. 1981.

Bd. 40 JOSEPH HENNINGER: Arabica Sacra. Aufsätze zur Religionsgeschichte Arabiens und seiner Randgebiete. Contributions à l'histoire religieuse de l'Arabie et de ses régions limitrophes. 347 Seiten. 1981.

Bd. 41 DANIEL VON ALLMEN: La famille de Dieu. La symbolique familiale dans le paulinisme. LXVII-330 pages, 27 planches. 1981.

Bd. 42 ADRIAN SCHENKER: Der Mäcbtige im Scbmelzofen des Mitleids. Eine Interpretation von 2 Sam 24. 92 Seiten. 1982.

Bd. 43 PAUL DESELAERS: Das Buch Tobit. Studien zu seiner Entstehung, Komposition und Theologie. 532 Seiten + Übersetzung 16 Seiten. 1982.

Bd. 44 PIERRE CASETTI: Gibt es ein Leben vor dem Tod? Eine Auslegung von Psalm 49. 315 Seiten. 1982 .

Bd. 45 FRANK-LOTHAR HOSSFELD: Der Dekalog. Seine späten Fassungen, die originale Komposition und seine Vorstufen. 308 Seiten. 1982. Vergriffen.

Bd. 46 ERIK HORNUNG: Der ägyptische Mythos von der Himmelskuh. Eine Ätiologie des Unvollkommenen. Unter Mitarbeit von Andreas Brodbeck, Hermann Schlögl und Elisabeth Staehelin und mit einem Beitrag von Gerhard Fecht. XII-129 Seiten, 10 Abbildungen. 1991. 2. ergänzte Auflage.

Bd. 47 PIERRE CHERIX: Le Concept de Notre Grande Puissance (CG VI, 4). Texte, remarques philologiques, traduction et notes. XIV-95 pages. 1982.

Bd. 48 JAN ASSMANN/WALTER BURKERT/FRITZ STOLZ: Funktionen und Leistungen des Mythos. Drei altorientalische Beispiele. 118 Seiten, 17 Abbildungen. 1982. Vergriffen.

Bd. 49 PIERRE AUFFRET: La sagesse a bâti sa maison. Etudes de structures littéraires dans l'Ancien Testament et spécialement dans les psaumes. 580 pages. 1982.

Bd. 50/1 DOMINIQUE BARTHÉLEMY: Critique textuelle de l'Ancien Testament. 1. Josué, Juges, Ruth, Samuel, Rois, Chroniques, Esdras, Néhémie, Esther. Rapport final du Comité pour l'analyse textuelle de l'Ancien Testament hébreu institué par l'Alliance Biblique Universelle, établi en coopération avec Alexander R. Hulst $t$, Norbert Lohfink, William D. McHardy, H. Peter Rüger, coéditeur, James A. Sanders, coéditeur. 812 pages. 1982. 
Bd. 50/2 DOMINIQUE BARTHÉLEMY: Critique textuelle de l'Ancien Testament. 2. Isaïe, Jérémie, Lamentations. Rapport final du Comité pour l'analyse textuelle de l'Ancien Testament hébreu institué par l'Alliance Biblique Universelle, établi en coopération avec Alexander R. Hulst $\dagger$, Norbert Lohfink, William D. McHardy, H. Peter Rüger, coéditeur, James A. Sanders, coéditeur. 1112 pages. 1986.

Bd. 51 JAN ASSMANN: Re und Amun. Die Krise des polytheistischen Weltbilds im Ägypten der 18.-20. Dynastie. XII-309 Seiten. 1983.

Bd. 52 MIRIAM LICHTHEIM : Late Egyptian Wisdom Literature in the International Context. A Study of Demotic Instructions. X-240 Seiten. 1983.

Bd. 53 URS WINTER: Frau und Göttin. Exegetische und ikonographische Studien zum weiblichen Gottesbild im Alten Israel und in dessen Umwelt. XVIII-928 Seiten, 520 Abbildungen. 1987. 2. Auflage. Mit einem Nachwort zur 2. Auflage.

Bd. 54 PAUL MAIBERGER: Topograpbische und bistoriscbe Untersucbungen zum Sinaiproblem. Worauf beruht die Identifizierung des Gabal Mūsā mit dem Sinai? 189 Seiten, 13 Tafeln. 1984.

Bd. 55 PETER FREI/KLAUS KOCH: Reichsidee und Reichsorganisation im Perserreich. 119 Seiten, 17 Abbildungen. 1984. Vergriffen. Neuauflage in Vorbereitung

Bd. 56 HANS-PETER MÜLLER: Vergleich und Metapher im Hobenlied. 59 Seiten. 1984.

Bd. 57 STEPHEN PISANO: Additions or Omissions in the Books of Samuel. The Significant Pluses and Minuses in the Massoretic, LXX and Qumran Texts. XIV-295 Seiten. 1984.

Bd. 58 ODO CAMPONOVO: Königtum, Königsherrschaft und Reich Gottes in den Frübjüdischen Schriften. XVI-492 Seiten. 1984.

Bd. 59 JAMES KARL HOFFMEIER: Sacred in the Vocabulary of Ancient Egypt. The Term DSR, with Special Reference to Dynasties I-XX. XXIV-281 Seiten, 24 Figures. 1985.

Bd. 60 CHRISTIAN HERRMANN: Formen für ägyptiscbe Fayencen. Katalog der Sammlung des Biblischen Instituts der Universität Freiburg Schweiz und einer Privatsammlung. XXVIII-199 Seiten. 1985.

Bd. 61 HELMUT ENGEL: Die Susanna-Erzäblung. Einleitung, Übersetzung und Kommentar zum Septuaginta-Text und zur Theodition-Bearbeitung. 205 Seiten + Anhang 11 Seiten. 1985.

Bd. 62 ERNST KUTSCH: Die cbronologischen Daten des Ezecbielbuches. 82 Seiten. 1985.

Bd. 63 MANFRED HUTTER: Altorientalische Vorstellungen von der Unterwelt. Literar- und religionsgeschichtliche Überlegungen zu «Nergal und Ereškigal». VIII-187 Seiten. 1985.

Bd. 64 HELGA WEIPPERT/KLAUS SEYBOLD/MANFRED WEIPPERT: Beiträge zur prophetischen Bildsprache in Israel und Assyrien. IX-93 Seiten. 1985.

Bd. 65 ABDEL-AZIZ FAHMY SADEK: Contribution à l'étude de l'Amdouat. Les variantes tardives du Livre de l'Amdouat dans les papyrus du Musée du Caire. XVI-400 pages, 175 illustrations. 1985.

Bd. 66 HANS-PETER STÄHLI: Solare Elemente im Jabweglauben des Aiten Testamentes. X-60 Seiten. 1985.

Bd. 67 OTHMAR KEEL/SILVIA SCHROER: Studien zu den Stempelsiegeln aus Palästina/Israel. Band I. 115 Seiten, 103 Abbildungen. 1985.

Bd. 68 WALTER BEYERLIN: Weisheitliche Vergewisserung mit Bezug auf den Zionskult. Studien zum 125. Psalm. 96 Seiten. 1985. 
Bd. 69 RAPHAEL VENTURA: Living in a City of the Dead. A Selection of Topographical and Administrative Terms in the Documents of the Theban Necropolis. XII-232 Seiten. 1986.

Bd. 70 CLEMENS LOCHER: Die Ehre einer Frau in Israel. Exegetische und rechtsvergleichende Studien zu Dtn 22, 13-21. XVIII-464 Seiten. 1986.

Bd. 71 HANS-PETER MATHYS: Liebe deinen Nächsten wie dich selbst. Untersuchungen zum alttestamentlichen Gebot der Nächstenliebe (Lev 19,18). XIV-196 Seiten. 1986. Vergriffen. Neuauflage in Vorbereitung.

Bd. 72 FRIEDRICH ABITZ: Ramses III. in den Gräbern seiner Söbne. 156 Seiten, 31 Abbildungen. 1986.

Bd. 73 DOMINIQUE BARTHÉLEMY/DAVID W. GOODING/JOHAN LUST/EMANUEL TOV: The Story of David and Goliath. 160 Seiten. 1986.

Bd. 74 SILVIA SCHROER: In Israel gab es Bilder. Nachrichten von darstellender Kunst im Alten Testament. XVI-553 Seiten, 146 Abbildungen. 1987.

Bd. 75 ALAN R. SCHULMAN: Ceremonial Execution and Public Rewards. Some Historical Scenes on New Kingdom Private Stelae. 296 Seiten, 41 Abbildungen. 1987.

Bd. 76 JOŽE KRAŠOVEC: La justice $(S ̣ d q)$ de Dieu dans la Bible bébraïque et l'interprétation juive et cbrétienne. 456 pages. 1988.

Bd. 77 HELMUT UTZSCHNEIDER: Das Heiligtum und das Gesetz. Studien zur Bedeutung der sinaitischen Heiligtumstexte (Ez 25-40; Lev 8-9). XIV-326 Seiten. 1988.

Bd. 78 BERNARD GOSSE: Isaie 13,1-14,23. Dans la tradition littéraire du livre d'Isaïe et dans la tradition des oracles contre les nations. 308 pages. 1988.

Bd. 79 INKE W. SCHUMACHER: Der Gott Sopdu - Der Herr der Fremdländer. XVI-364 Seiten, 6 Abbildungen. 1988.

Bd. 80 HELLMUT BRUNNER: Das börende Herz. Kleine Schriften zur Religions- und Geistesgeschichte Ägyptens. Herausgegeben von Wolfgang Röllig. 449 Seiten, 55 Abbildungen. 1988.

Bd. 81 WALTER BEYERLIN: Bleilot, Brecheisen oder was sonst? Revision einer Amos-Vision. 68 Seiten. 1988.

Bd. 82 MANFRED HUTTER: Bebexung, Entsïbnung und Heilung. Das Ritual der Tunnawiya für ein Königspaar aus mittelhethitischer Zeit (KBo XXI 1 - KUB IX 34 - KBo XXI 6). 186 Seiten. 1988.

Bd. 83 RAPHAEL GIVEON: Scarabs from Recent Excavations in Israel. 114 Seiten, 9 Tafeln. 1988.

Bd. 84 MIRIAM LICHTHEIM : Ancient Egyptian Autobiograpbies chiefly of the Middle Kingdom. A Study and an Anthology. 200 Seiten, 10 Seiten Abbildungen. 1988.

Bd. 85 ECKART OTTO: Rechtsgeschichte der Redaktionen im Kodex Ešnunna und im "Bundesbuch». Eine redaktionsgeschichtliche und rechtsvergleichende Studie zu altbabylonischen und altisraelitischen Rechtsüberlieferungen. 220 Seiten. 1989.

Bd. 86 ANDRZEJ NIWIŃSKI: Studies on the Illustrated Theban Funerary Papyri of the 11th and 10th Centuries B.C. 488 Seiten, 80 Seiten Tafeln. 1989.

Bd. 87 URSULA SEIDL: Die babylonischen Kudurru-Reliefs. Symbole mesopotamischer Gottheiten. 236 Seiten, 33 Tafeln und 2 Tabellen. 1989.

Bd. 88 OTHMAR KEEL/HILDI KEEL-LEU/SILVIA SCHROER: Studien zu den Stempelsiegeln aus Palästina/Israel. Band II. 364 Seiten, 652 Abbildungen. 1989.

Bd. 89 FRIEDRICH ABITZ: Baugeschichte und Dekoration des Grabes Ramses' VI. 202 Seiten, 39 Abbildungen. 1989. 
Bd. 90 JOSEPH HENNINGER SVD: Arabica varia. Aufsätze zur Kulturgeschichte Arabiens und seiner Randgebiete. Contributions à l'histoire culturelle de l'Arabie et de ses régions limitrophes. 504 Seiten. 1989.

Bd. 91 GEORG FISCHER : Jabwe unser Gott. Sprache, Aufbau und Erzähltechnik in der Berufung des Mose (Ex. 3-4). 276 Seiten. 1989.

Bd. 92 MARK A. O'BRIEN: The Deuteronomistic History Hypothesis: A Reassessment. 340 Seiten. 1989.

Bd. 93 WALTER BEYERLIN: Reflexe der Amosvisionen im Jeremiabuch. 120 Seiten. 1989.

Bd. 94 ENZO CORTESE: Josua 13-21. Ein priesterschriftlicher Abschnitt im deuteronomistischen Geschichtswerk. 136 Seiten. 1990.

Bd. 95 ERIK HORNUNG (Herausgeber): Zum Bild Ägyptens im Mittelalter und in der Renaissance. Comment se représente-t-on l'Egypte au Moyen Age et à la Renaissance. 268 Seiten. 1990.

Bd. 96 ANDRÉ WIESE: Zum Bild des Königs auf ägyptischen Siegelamuletten. 264 Seiten. 1990.

Bd. 97 WOLFGANG ZWICKEL: Räucherkult und Räuchergeräte. Exegetische und archäologische Studien zum Räucheropfer im Alten Testament. 372 Seiten. 1990.

Bd. 98 AARON SCHART: Mose und Israel im Konflikt. Eine redaktionsgeschichtliche Studie zu den Wüstenerzählungen. 296 Seiten. 1990.

Bd. 99 THOMAS RÖMER : Israels Väter. Untersuchungen zur Väterthematik im Deuteronomium und in der deuteronomistischen Tradition. 664 Seiten. 1990.

Bd. 100 O'THMAR KEEL/MENAKHEM SHUVAL/CHRISTOPH UEHLINGER : Studien zu den Stempelsiegeln aus Palästinal Israel. Band III. Die Frühe Eisenzeit. Ein Workshop. XIV-456 Seiten. XXII Tafeln. 1990.

Bd. 101 CHRISTOPH UEHLINGER: Weltreich und «eine Rede». Eine neue Deutung der sogenannten Turmbauerzählung (Gen 11,1-9). XVI-654 Seiten. 1990.

Bd. 102 BENJAMIN SASS: Studia Alphabetica. On the Origin and Early History of the Northwest Semitic, South Semitic and Greek Alphabets. X-120 Seiten. 16 Seiten Abbildungen. 2 Tabellen. 1991.

Bd. 103 ADRIAN SCHENKER: Text und Sinn im Alten Testament. Textgeschichtliche und bibeltheologische Studien. VIII-312 Seiten. 1991.

Bd. 104 DANIEL BODI: The Book of Ezekiel and the Poem of Erra. IV-332 Seiten. 1991.

Bd. 105 YUICHI OSUMI: Die Kompositionsgeschicbte des Bundesbuches Exodus 20,22b-23,33. XII-284 Seiten. 1991.

Bd. 106 RUDOLF WERNER: Kleine Einfübrung ins Hieroglyphen-Luwische. XII-112 Seiten. 1991.

Bd. 107 THOMAS STAUBLI: Das Image der Nomaden. Ca. 420 Seiten. 1991.

Bd. 108 MOSHÉ ANBAR: Les tribus amurrites de Mari. VIII-256 Seiten. 1991. 


\section{Résumé}

A Tell Harīī sur le Moyen-Euphrate, site de l'ancienne ville de Mari, 27 saisons de fouilles ont été dirigées jusqu'à maintenant, au cours desquelles 20000 tablettes et fragments de tablettes ont été trouvés, la plupart datant du milieu du XVIII siècle avant notre ère, l'époque de Hammurabi, roi de Babylonie. L'espace géographique qui forme le cadre où se sont déroulés les événements décrits dans ces documents va du golfe Persique jusqu'à la Méditerranée. L'espace qu'ils décrivent est riche en événements et en vicissitudes politiques, auxquels ont pris part des dizaines de grands et de petits rois, des rois de grands royaumes et de petites villes-royaumes. Dans les documents se reflètent à la fois les événements de grande portée et la vie journalière de la couche gouvernante ainsi que des simples citoyens.

Les archives de Mari contiennent par ailleurs une riche information sur des tribus semi-nomades, qui appartiennent à la branche ouest des peuples sémitiques, qualifiés d'Amurrites. L'étude commence par une revue de I'histoire de la recherche sur les tribus amurrites puis décrit la chronologie et I'histoire de Mari à l'époque des dynasties amurrites. Par la suite sont examinés les divers aspects qui concernent ces tribus, à savoir leur organisation, leur distribution géographique, leurs dirigeants, leur économie, leurs relations avec les autorités et enfin leur origine.

\section{Summary}

At Tell Hariri, on the Middle-Euphrates, the site of the ancient city of Mari, twenty-seven seasons of excavations have so far been carried out, in the course of which about 20000 tablets and tablet fragments have been retrieved, the overwhelming majority dating to the eighteenth century B.C.E., the time of Hammurabi, king of Babylon. The texts cast light on a geographical region extending from the Persian Gulf as far as the Mediterranean. The period covered by these texts was highly eventful with numerous political upheavals in which a role was played by monarchs of powerful kingdoms and rulers of small city-states. Political events of world-wide impact, as well as the everyday life of the ruling classes and the common people, are reflected in the texts.

The Mari archive also contains a wealth of information about semi-nomadic tribes affiliated with that western branch of the Semitic peoples called "Amorites». The study begins with a review of the history of research on the Amorite tribes, after which the chronology and the history of Mari at the period of the Amorite dynasties is described. Then different aspects of these tribes are studied: tribal organization, geographical distribution, tribal leadership, tribal economy, the relationship between the tribes and the ruling power and finally the origin of the Amorite tribes. 